\title{
Lepton production in ice by scattering of astrophysical neutrinos at high energies
}

\author{
Dissertation \\ zur Erlangung des Doktorgrades \\ der Mathematisch-Naturwissenschaftlichen Fakultäten \\ der Georg-August-Universität zu Göttingen
}

vorgelegt von

Christian Hettlage

aus Münster/Westfalen

Göttingen, den 1.9.2005 
D 7

Referent:

Prof. Dr. Karl Mannheim

Korreferent:

Prof. Dr. Klaus Beuermann

Tag der mündlichen Prüfung: 23. September 2005 


\section{Contents}

1 Introduction $\quad 5$

2 Some lepton physics 11

2.1 Neutrino properties and oscillations . . . . . . . . . . . . . 11

2.2 Neutrino-nucleon interactions . . . . . . . . . . . . . 18

2.3 Neutrino-electron interactions . . . . . . . . . . . . . . . . 22

2.4 Lepton energy losses and decay . . . . . . . . . . . . . 26

3 Propagation analysis 31

3.1 The cascade equations . . . . . . . . . . . . . . . . 31

3.2 Integral transformations . . . . . . . . . . . . . . 36

3.2.1 Formal approach . . . . . . . . . . . . 36

$3.2 .2 \quad$ Approximate approach . . . . . . . . . . . . . . 43

3.3 Discretizing the cascade equation . . . . . . . . . . . 46

3.4 Iteration . . . . . . . . . . . . . . . . . . . 51

4 Neutrino detection $\quad 61$

4.1 Neutrino detectors . . . . . . . . . . . . . . . 61

4.2 Generic water-based Čerenkov detector . . . . . . . . . . 65

5 Astrophysical neutrino sources $\quad 69$

5.1 The Sun . . . . . . . . . . . . . . . . . 70

5.2 From source spectra to event rates . . . . . . . . . . . 79

5.3 Galactic sources . . . . . . . . . . . . . . . . . . . . 98

5.4 Extragalactic sources . . . . . . . . . . . . . . . . . 102

$5.5 \quad$ Flux limits . . . . . . . . . . . . . . . . . . . . . . . . . . . . . 114 
6 Tomography of the inner Earth 121

6.1 Preliminary Reference Earth Model . . . . . . . . . . . . . . . 122

6.2 Inverse Radon transform . . . . . . . . . . . . . . . . . . . 124

6.3 Weighing the Earth . . . . . . . . . . . . . . . . . . . . . 132

$\begin{array}{lll}7 & \text { Summary and outlook } & 147\end{array}$

$\begin{array}{ll}\text { A Software } & 151\end{array}$

A.1 TEMPEST . . . . . . . . . . . . . . . . . . . . . . . . 151

A.1.1 Requirements . . . . . . . . . . . . . 151

A.1.2 Installation . . . . . . . . . . . . . . 151

A.1.3 Running the program . . . . . . . . . . . . . . . 153

A.1.4 Adding a new source . . . . . . . . . . . . . 156

B Event rates and $Z$ factor inaccuracies $\quad 157$

B.1 Astrophysical neutrino event rates . . . . . . . . . . . . . . 157

B.2 Inaccuracy of the $Z$ factor $\ldots \ldots \ldots$. . . . . . . . . . . . . . . . . . . . 


\section{Chapter 1}

\section{Introduction}

In order to explain the continuous energy spectrum of the electrons emitted in beta decays, Pauli in 1930 proposed the existence of a new neutral particle, [48] which later came to be known as neutrino. However, it took until 1953 before the (electron) neutrino actually could be detected by means of inverse beta decays induced by neutrinos from a nuclear reactor. [169] In 1962, an inverse beta decay experiment at Brookhaven established the existence of the muon neutrino. [60] Energy and momentum conservation as well as theoretical considerations provided massive evidence for the existence of a third neutrino, the tau neutrino, and indeed this neutrino was detected by the DONUT experiment. [120]

Neutrinos have no charge, and if they possess a magnetic moment, its value must be very small. [70] In addition, they don't take part in strong interactions, so that they are affected by weak interactions only. This has the important consequence that their cross section is very small. Indeed, at energies of several hundred $\mathrm{keV}$, it just has a value of about $10^{-45} \mathrm{~cm}^{2}$ per nucleon. To illustrate the point, we note that in general none of the $7 \times 10^{10}$ solar neutrinos crossing each square centimeter of our body each second interacts with any nucleon within us. [94]

Hence from the point of view of an astrophysicist, neutrinos constitute an almost perfect means of observation: Once they have left their source, they are neither absorbed nor deflected by magnetic fields, and they (probably) don't decay on their flight to Earth. The measurement of a neutrino spectrum and the direction of arrival thus allows immediate conclusions concerning the source. (For the sake of completeness, we should add that on cosmological scales, neutrinos of course suffer from the usual energy degradation due to 
the expansion of the universe, and that their flavor might change, as shown below.) Hence astrophysical neutrinos might provide a valuable new window to the universe - if they exist.

But two astrophysical neutrino sources have been found already. Firstly, in 1968, the Homestake experiment recorded the neutrino flux resulting from nuclear fusion in the solar core. [61] Soon it became apparent that there was a discrepancy between the predicted and the actually detected flux, but it remained a mystery for the next three decades whether this "solar neutrino problem" was caused by a flaw in the solar models or in the particle physics description of the neutrino. Secondly, the supernova SN1987 A, which blazed off in the Large Magellanic Cloud in Februrary 1987, was seen as a few seconds long neutrino pulse in the Baksan, IMB, and Kamiokande detectors. [168]

In addition, there is conclusive circumstantial evidence for the existence of astrophysical neutrinos with energies exceeding those of fusion neutrinos by several orders of magnitude. This rests on the fact that cosmic rays contain high energy protons. Such protons may interact with photons or other protons to give pions, which subsequently decay into a muon and a muon neutrino. The former is unstable as well, and its decay results in the creation of an electron, an electron neutrino, and yet another muon neutrino. Summing up, the proton interactions thus yield a neutrino flux with the flavor ratio $\nu_{e}: \nu_{\mu}: \nu_{\tau}=1: 2: 0$, and this ratio usually is assumed for the production of astrophysical neutrinos.

The preceding paragraph has made use of an important convention, which will be employed throughout this work: If nothing else is stated, no distinction is made between a particle and its corresponding antiparticle. Thus, for example, the symbol $\nu_{\mu}$ refers to both the muon neutrino and the muon antineutrino, and the flux $\phi_{\nu_{\mu}}$ denotes the sum of the neutrino and antineutrino fluxes.

A plausible acceleration mechanism for protons in astrophysical sources is provided by the so-called Fermi acceleration. It basically adds energy by repeated reflections of the accelerated particle. The principle can be understood in terms of a table tennis ball moving between two rackets approaching each other: Each time the ball hits either of the rackets, its speed is increased by twice that of the racket. In astrophysical circumstances, such an acceleration may occur at shock fronts, and hence, for example, supernova remnants and gamma-ray bursts are good candidates for high-energy proton and hence neutrino sources. 
Apart from hadronic neutrino sources, in which protons are accelerated and subsequently produce neutrinos, there might be non-hadronic sources. These are of particular interest, as they would typically involve some physics beyond the standard model. Decaying supermassive relic particles created shortly after the big bang (cf., e.g., [43]) might be an example.

Given the flavor ratio 1:2:0 quoted above, it is tempting to assume that there should be no astrophysical tau neutrinos. But our picture of neutrino physics isn't complete yet. One of the key questions raised by the discovery of the neutrino was whether it actually has any mass and, if so, how large this mass is. Even today, the second part of the question hasn't been answered, and one can only conclude from tritium beta decay experiments that the electron neutrino mass must be less than about $3 \mathrm{eV}$. The corresponding lower limits for the muon and tau neutrino mass are considerably less restrictive. [70] It has been claimed that observations of the cosmic ray background together with Sloan Digital Sky Survey measurements of intergalactic gas backlit by quasars constrain the sum of neutrino masses for all three flavors to be less than $0.42 \mathrm{eV}$. [184]

But an answer to the more important first part of the question has been given. The measurement of the atmospheric electron and muon neutrinos by means of the SuperKamiokande detector showed that there was a deficit in upward moving muon neutrinos, which had to cross the Earth prior to reaching the detector. The detailed angular distribution of the flux proved to be consistent with the explanation that the upward moving neutrinos change the flavor on their way. [81] These flavor oscillations, which have been confirmed for solar neutrinos by SNO later on [5] and thus are the explanation of the solar neutrino problem, require mass differences between the different flavors that are less than $1 \mathrm{eV}$, but non-vanishing. On astrophysical scales, they alter the neutrino flavor ratio from its initial value 1:2:0 to 1:1:1. [27] Astrophysical sources accordingly offer the prospect of a tau neutrino detection.

Although the fact that neutrinos interact only weakly may appear like a blessing for astrophysicists, it must at the same time be considered as somewhat of a curse. After all, small cross sections necessarily imply small detection rates. Concerning astrophysical neutrino sources with energies of $100 \mathrm{GeV}$ and more, this means in practice that one needs a detector volume of at least a cubic kilometer in order to be able to see any source. Hence the classical method of putting some target matter (such as gallium or water) in a huge tank obviously becomes unfeasible. 
Instead, one may use naturally occuring reservoirs of water like the antarctic ice or the Mediterranean Sea, employing the Čerenkov effect: If a neutrino scatters inelastically on a nucleon, a charged lepton may be created, which, being faster than light in the water, emits an electromagnetic wave. Alternatively, one may look, e.g., for acoustic signals due to the almost instantaneous deposition of heat during a hadronic shower initiated by a neutrino interaction. In addition, air Čerenkov telescopes like Auger might detect ultrahigh-energy neutrinos.

The cross sections for neutrino-nucleon scatterings increase with energy, and for sufficiently high energies the Earth becomes opaque for neutrinos. For electron and muon neutrinos this implies that the overall number of neutrinos is diminished and that there is an additional energy degradation. For tau neutrinos, on the other hand, only the latter is true; their overall number remains constant. This suggests that tau neutrinos play a more imprtant role when it comes to event rates, but we shall see that this is not the case.

Hence a description of neutrino propagation through the Earth is called for. This work investigates several methods for dealing with the necessary set of integro-differential equations, and thus shows that the detailed understanding of an observed neutrino spectrum requires a combined treatment of all three flavors.

While at first sight neutrino absorption and regeneration in the Earth may appear like a complication, which must (and can) be overcome, it also has to be regarded as a chance: For an isotropic initial neutrino flux, the angular distribution of the neutrino events should allow a tomography and thus similarly to seismic waves provide some insight into the terrestrial density structure, as we will investigate in full detail.

To be more specific, this work starts with outlining the necessary concepts of lepton physics in Chapter 2. The neutrino-nucleon cross sections are obtained, the relevant formulae for lepton decays and energy losses are provided, and neutrino flavor oscillations are discussed. In Chapter 3 these results are applied to the neutrino propagation. The required cascade equations are derived, and integral transforms, discretization, and iteration are discussed as possible methods of solution, where the method of iteration is shown to allow an accurate combined treatment of all three neutrino flavors. Chapter 4 gives an overview over various neutrino detectors and then proceeds to investigate a generic detector, which is used throughout this work.

Using the results from Chapters 3 and 4 , the implications of neutrino absorption and regeneration in the Earth are discussed in full detail for a 
generic input spectrum, and are subsequently evaluated for a huge variety of suggested neutrino sources. In addition, the Sun is discussed as a a high energy neutrino source, and it is investigated whether future solar neutrino measurements might allow inferences regarding the neutrino flavor oscillation parameters.

Following a brief account of seismic waves, the implications of neutrino propagation for a tomography of the inner Earth and a gravity-independent determination of the mass of the Earth are considered in Chapter 6. Finally, Chapter 7 provides a brief summary and outlook. 


\section{Chapter 2}

\section{Some lepton physics}

\subsection{Neutrino detection, properties, and oscil- lations}

If the $\beta^{-}$decay resulted in the creation of two particles, i.e. if it could be fully described by the reaction

$$
{ }_{Z}^{A} X \longrightarrow{ }_{Z+1}^{A} Y+e^{-},
$$

the product nucleus and electron both should have a fixed energy. However, a countinuous energy spectrum is observed (cf., e.g., [44]), and in order to solve this problem, Pauli in 1930 reluctantly suggested the existence of a hitherto unknown particle, which in hindsight would best be described as a mixture of neutron and neutrino. [48]

It turned out later that the missing particle must be uncharged, doesn't take part in strong interactions, and has almost no mass, and it came to be known as an electron antineutrino. The name "neutrino" actually was born when Fermi after his report on the detection of the neutron was asked by a student whether Pauli's proposed particle and the neutron were the same and gave the reply that il neutrone di Pauli è molto più piccolo, cio è un neutrino. [85]

As neutrinos interact weakly only, their detection proved a tremendous task, which wasn't accomplished before 1953, when their existence was proved by means of inverse beta decays induced by neutrinos from the Savannah River nuclear reactor. [169] In 1962, experiments at Brookhaven showed that the reactions of neutrinos from pion decay with protons yield muons, but 
no electrons. [60] Hence apart from the electron neutrino there must exist a second kind of neutrino, now known as the muon neutrino.

After the detection of the tauon in 1975 [162], theoretical as well as experimental evidence strongly suggested the existence of yet another neutrino, called the tau neutrino. 1 It was finally observed by the DONUT experiment, which detected tauons produced in tau neutrino interactions. [120]

Hence from a modern point of view there exist three charged leptons and three corresponding neutrinos, which can be grouped in pairs ("generations") as

$$
\left(\begin{array}{c}
\nu_{e} \\
e
\end{array}\right),\left(\begin{array}{c}
\nu_{\mu} \\
\mu
\end{array}\right),\left(\begin{array}{c}
\nu_{\tau} \\
\tau
\end{array}\right)
$$

and which all have an antiparticle. Measurements of the $\mathrm{Z}^{0}$ branching ratios [9] and cosmological observations based on nucleosynthesis and the ${ }^{4} \mathrm{He}$ abundance [179] rule out the existence of further neutrino flavors with small mass, at least if the neutrinos take part in weak interactions. (See [45] for a more detailed discussion.)

For each flavor $l$, one may define a lepton number $L_{l}$ by

$$
L_{l} \equiv N\left(l^{-}\right)+N\left(\nu_{l}\right)-N\left(l^{+}\right)-N\left(\bar{\nu}_{l}\right)
$$

where $N$ denotes the number of particles in a given reaction. These lepton numbers are conserved in weak, strong, and electromagnetic interactions (but see below). For convenience, the value of various quantities are listed for the neutrinos and charged leptons in Table 2.1.

As can be seen from the table, the values of the neutrino masses could not be established yet, and a long-standing question of particle physics was whether neutrinos are massless. In order to see what consequences a neutrino mass might have, let us limit ourselves to the case of two neutrino flavors $l$, $l^{\prime}$ (with $l \neq l^{\prime}$ ) first, following the analysis of [45].

Hence let us assume that there are two neutrino masses $m_{k}(k=1,2)$. Then the time development of the respective mass eigenstates $\left|\nu_{k}(t)\right\rangle$ is given by

$$
\left|\nu_{k}(t)\right\rangle=e^{i E_{k} t}\left|\nu_{k}(0)\right\rangle
$$

\footnotetext{
${ }^{1}$ The $\tau$ is called both "tau" and "tauon" in the literature, and similarly the $\nu_{\tau}$ is known as both "tau neutrino" and "tauon neutrino", respectively. We use the names "tauon" and "tau neutrino". There is no deeper meaning in this particular choice.
} 


\begin{tabular}{|c|c|c|c|}
\hline \multirow[t]{2}{*}{ quantity } & \multicolumn{3}{|c|}{ value } \\
\hline & $e$ & $\mu$ & $\tau$ \\
\hline \multirow{6}{*}{$\begin{array}{l}\text { mass }(\mathrm{MeV}) \\
\text { charge }(\mathrm{C}) \\
\text { spin } \\
\text { magnetic moment } \\
\text { lifetime }(\mathrm{s}) \\
\end{array}$} & 0.51100 & 105,658 & 1777 \\
\hline & $-1.6022 \times 10^{-19}$ & $-1.6022 \times 10^{-19}$ & $-1.6022 \times 10^{-19}$ \\
\hline & & & \\
\hline & $1.0011597 \mu_{\mathrm{B}}$ & $1.0011659 \mu_{\mu}$ & $0.948 \mu_{\tau}<\mu<1.058 \mu_{\tau}$ \\
\hline & $>1.4 \times 10^{34}$ & $2.1970 \times 10^{-6}$ & $(290.6 \pm 1.1) \times 10^{-15}$ \\
\hline & $\nu_{e}$ & $\nu_{\mu}$ & $\nu_{\tau}$ \\
\hline mass $(\mathrm{MeV})$ & $<3 \times 10^{-6}$ & $<0.19$ & $<18.2$ \\
\hline charge $(\mathrm{C})$ & 0 & 0 & 0 \\
\hline spin & & $\frac{1}{2}$ & \\
\hline magnetic moment & $<1.0 \times 10^{-10} \mu_{\mathrm{B}}$ & $<6.8 \times 10^{-10} \mu_{\mathrm{B}}$ & $<3.9 \times 10^{-7} \mu_{\mathrm{B}}$ \\
\hline lifetime/mass $(\mathrm{s} / \mathrm{eV})$ & $>7 \times 10^{9}$ & $>15.4$ & (no reliable lower limit) \\
\hline
\end{tabular}

Table 2.1: Values for the mass, charge, spin, magnetic moment, and lifetime of the leptons. The corresponding antiparticles have the same masses, spins, and lifetimes; their charges and magnetic moments have the same absolute values, but the opposite sign. $\mu_{\mathrm{B}}$ denotes the Bohr magneton, and $\mu_{l}$ is defined as $\left(m_{e} / m_{l}\right) \mu_{\mathrm{B}}$. More precise values can be found in [70].

where $E_{k}=\left(p^{2}+m_{k}^{2}\right)^{1 / 2}$ denotes the neutrino energy. (Note that here and in the following, we use natural units, i.e. $\hbar=c=1$.) There is no need to assume that the state vectors describing a $\nu_{l}$ or $\nu_{l^{\prime}}$ are parallel to the state vectors $\left|\nu_{k}\right\rangle$. However, as $\left|\nu_{l}\right\rangle$ and $\left|\nu_{l^{\prime}}\right\rangle$ on the one, and $\left|\nu_{1}(0)\right\rangle$ and $\left|\nu_{2}(0)\right\rangle$ on the other hand are orthonormal, the first may be obtained from the latter by means of a unitary transformation. Ignoring irrelevant phase factors, we can thus state without loss of generality that

$$
\left(\begin{array}{l}
\left|\nu_{l}\right\rangle \\
\left|\nu_{l^{\prime}}\right\rangle
\end{array}\right)=\left(\begin{array}{cc}
\cos \theta & \sin \theta \\
-\sin \theta & \cos \theta
\end{array}\right)\left(\begin{array}{l}
\left|\nu_{1}(0)\right\rangle \\
\left|\nu_{2}(0)\right\rangle
\end{array}\right)
$$

where the value of the mixing angle $\theta$ must be obtained from experiment. Hence the time evolution of a state $|\psi(t)\rangle$ which starts off as a neutrino $\nu_{l}$, $|\psi(0)\rangle=\left|\nu_{l}\right\rangle$, has the form

$$
|\psi(t)\rangle=\cos \theta e^{i E_{1} t}\left|\nu_{1}(0)\right\rangle+\sin \theta e^{i E_{2} t}\left|\nu_{2}(0)\right\rangle .
$$

The probability $P_{\nu_{l} \rightarrow \nu_{l^{\prime}}}(t)$ to observe $|\psi(t)\rangle$ as a neutrino $\nu_{l^{\prime}}$ has the value $\left|\left\langle\nu_{l^{\prime}} \mid \psi(t)\right\rangle\right|^{2}$. Expressing $\left\langle\nu_{l^{\prime}}\right|$ in terms of the $\left\langle\nu_{k}(0)\right|$ by means of Eq. 2.1 and using the orthonormality relation $\left\langle\nu_{k} \mid \nu_{k^{\prime}}\right\rangle=\delta_{k k^{\prime}}$, we see that $P_{\nu_{l} \rightarrow \nu_{l^{\prime}}}$ can be 
written as

$$
P_{\nu_{l} \rightarrow \nu_{l^{\prime}}}(t)=\sin ^{2} 2 \theta \sin ^{2}\left(\frac{\left(E_{2}-E_{1}\right) t}{2}\right) .
$$

Given the definition of $|\psi\rangle$, it is evident that $P_{\nu_{l} \rightarrow \nu_{l^{\prime}}}(t)$ can be interpreted as the probability that at the time $t$ a neutrino of the initial flavor $l$ appears as a neutrino of the other flavor $l^{\prime}$, so that Eq. 2.2 describes a neutrino flavor oscillation.

As the neutrino masses are negligibly small, we may approximate the energies $E_{k}$ by their Taylor expansion to first order,

$$
E_{k}=\sqrt{p^{2}+m_{k}^{2}} \approx p+\frac{1}{2 E_{k}} \cdot m_{k}^{2} \approx p+\frac{m_{k}^{2}}{2 E},
$$

where the energy $E$ in the last expression may be either $E_{1}$ or $E_{2}$, as the difference between the two can be neglected. Using this approximation and taking into account that for all practical means neutrinos move with the speed of light, we can rewrite Eq. 2.2 in terms of the energy and the path length $x$ as

$$
P_{\nu_{l} \rightarrow \nu_{l^{\prime}}}(E, x)=\sin ^{2} 2 \theta \sin ^{2}\left(\frac{m_{2}^{2}-m_{1}^{2}}{4} \frac{x}{E}\right),
$$

and together with the definition $\Delta m_{i k}^{2} \equiv m_{i}^{2}-m_{k}^{2}$ we get the expression

$$
L_{\mathrm{osc}}(E)=\frac{4 \pi E}{\Delta m_{21}^{2}}=2480\left(\frac{E}{1 \mathrm{GeV}}\right)\left(\frac{\Delta m_{21}^{2}}{1 \mathrm{eV}^{2}}\right)^{-1} \mathrm{~m}
$$

for the period length $L_{\text {osc }}$ of the oscillation, which corresponds to a phase difference of $\pi$. We see that $L_{\text {osc }}$ depends on the difference of the mass squares and that a vanishing mass difference implies that there can be no oscillations. In other words, if there are such oscillations, neutrinos must have a finite mass.

For the case of three neutrinos, the unitary transformation between the mass eigenstates $\left|\nu_{1,2,3}\right\rangle$ and the flavor eigenstates $\left|\nu_{e, \mu, \tau}\right\rangle$ is represented by a $3 \times 3$ matrix $U$, which can be parametrized as [135]

$$
U=\left(\begin{array}{ccc}
c_{13} c_{12} & s_{12} c_{13} & s_{13} \\
-s_{12} c_{23}-s_{23} s_{13} c_{12} & c_{23} c_{12}-s_{23} s_{13} s_{12} & s_{23} c_{13} \\
s_{23} s_{12}-s_{13} c_{23} c_{12} & -s_{23} c_{12}-s_{13} s_{12} c_{23} & c_{23} c_{13}
\end{array}\right)
$$


with $s_{i k} \equiv \sin \theta_{i k}$ and $c_{i k} \equiv \cos \theta_{i k}$. The calculation of the corresponding transition probabilities $P_{\nu_{l} \rightarrow \nu_{l^{\prime}}}$ is essentially the same as that for the two flavor case and yields the result [42]

$$
P_{\nu_{l} \rightarrow \nu_{l^{\prime}}}(E, x)=\left|\delta_{l l^{\prime}}+\sum_{k=2}^{3} U_{l^{\prime} k} U_{l k}^{*}\left[\exp \left(-i \frac{\Delta m_{k 1}^{2}}{2} \frac{x}{E}\right)-1\right]\right|^{2} .
$$

For the sake of completeness we note that this formula can be generalized to the case of $n>3$ flavors by simply replacing the " 3 " overneath the sum by $n$. However, the existence of a fourth (sterile) neutrino flavor is disfavored by the current observational data. [135]

The first evidence that there might be neutrino flavor oscillations was provided by the Homestake experiment, which detected a flux of solar electron neutrinos which was below that predicted by solar models, hence giving rise to the "solar neutrino problem". [61] The proof was finally achieved by the SuperKamiokande experiment, which measured the flux of atmospheric neutrinos as a function of the nadir angle and showed that there was a deficit in upward moving muon neutrinos, which had to cross the Earth before reaching the detector. [81]

This finding was reiterated by the Sudbury Neutrino Observatory (SNO). Contrary to the previous solar neutrino experiments, SNO could detect neutrinos of all flavors and thus was able to show that the overall solar neutrino flux is indeed consistent with the value predicted by solar models and that the solar electron neutrinos oscillate into either muon or tau neutrinos on their way to Earth. [5] Thus the lepton numbers $L_{l}$ are not conserved during neutrino propagation.

A complete description of neutrino oscillations obviously requires the knowledge of the three mixing angles $\theta_{12}, \theta_{13}, \theta_{23}$ and the two mass square differences $\Delta m_{12}^{2}, \Delta m_{13}^{2}$. These can be extracted from the observation of neutrinos from the Sun, the atmosphere, and accelerators; recent values are given in Table 2.2 .

Neutrino oscillations are of considerable importance for neutrino astrophysics. To see why, we note that neutrinos produced in the various proposed astrophysical neutrino sources usually result from the decay of a charged pion,

$$
\pi \longrightarrow \mu+\nu_{\nu},
$$

where we follow the usual convention in this work and don't explicitly distinguish between particles and antiparticles. The muon subsequently decays 


\begin{tabular}{|l|lccc|}
\hline parameter & best fit & $2 \sigma$ interval & $3 \sigma$ interval & $4 \sigma$ interval \\
\hline$\Delta m_{21}^{2}\left(10^{-5} \mathrm{eV}^{2}\right)$ & 8.1 & $7.5-8.7$ & $7.2-9.1$ & $7.0-9.4$ \\
$\Delta m_{31}^{2}\left(10^{-3} \mathrm{eV}^{2}\right)$ & 2.2 & $1.7-2.9$ & $1.4-3.3$ & $1.1-3.7$ \\
$\sin ^{2} \theta_{12}$ & 0.30 & $0.25-0.34$ & $0.23-0.38$ & $0.21-0.41$ \\
$\sin ^{2} \theta_{23}$ & 0.50 & $0.38-0.64$ & $0.34-0.68$ & $0.30-0.72$ \\
$\sin ^{2} \theta_{13}$ & 0.000 & $\leqslant 0.028$ & $\leqslant 0.047$ & $\leqslant 0.068$ \\
\hline
\end{tabular}

Table 2.2: Neutrino oscillation parameters from a combined analysis of neutrinos from the Sun, the atmosphere, and accelerators, as given by [135].

via

$$
\mu \longrightarrow e+\nu_{e}+\nu_{\mu},
$$

and summing up Eqs. 2.6 and 2.7, we see that one should expect a flavor ratio

$$
\nu_{e}: \nu_{\mu}: \nu_{\tau} \approx 1: 2: 0 \quad \text { (at source) }
$$

from astrophysical sources. But as astronomical distances are long compared to the oscillation length (Eq. 2.4), flavor oscillations have to be taken into account, which are, however, smeared out, so that the transition probabilities are independent of the distance between source and Earth. As shown in [27], this implies that the flavor ratio should have the value

$$
\nu_{e}: \nu_{\mu}: \nu_{\tau} \approx 1: 1: 1 \quad(\text { at Earth) }
$$

when the neutrinos reach the Earth. Accordingly, a tau neutrino flux must be expected even though virtually no tau neutrinos are produced in the sources. Hence when neutrinos of astrophysical origin are considered, all three flavors must be taken into account.

It has recently been pointed out in [113] that pion and muon energy losses prior to the decay might substantially alter the initial neutrino flavor ratio and that therefore the flavor ratios at the source and at the Earth might reach a value of 0:1:0 and 1:1.8:1.8, respectively. However, we shall continue to employ the standard ratio 1:1:1 for the discussion of astrophysical sources throughout this work, keeping in mind that the actual ratio might favor muon and tau neutrinos.

So far, we have considered neutrino oscillations in vacuum only. If instead the neutrinos move through matter, one has to take into account that while 
neutrinos of any flavor may interact with electrons via the exchange of a $Z^{0}$ boson, only electron neutrinos can in addition interact via the exchange of a $\mathrm{W}^{+}$boson. This leads to an additional phase factor for the time evolution of a state vector describing an electron neutrino and is known as the MikheyevSmirnov-Wolfenstein (MSW) effect. [196, 147]

If we restrict ourselves once again to the case of two flavors (one of them being the electron neutrino), the MSW effect leads to the transition probability [45, 177]

$$
P_{\nu_{e} \rightarrow \nu_{l^{\prime}}}(E, x)=\sin ^{2} 2 \theta_{\mathrm{MSW}} \sin ^{2}\left(\pi \cdot \frac{x}{L_{\mathrm{MSW}}}\right)
$$

where we have assumed a constant density and where the mixing angle $\theta_{\mathrm{MSW}}$ and oscillation length $L_{\mathrm{MSW}}$ are given by

$$
\sin 2 \theta_{\mathrm{MSW}} \equiv \frac{L_{\mathrm{MSW}}}{L_{\mathrm{osc}}} \sin 2 \theta
$$

and

$$
\tan 2 \theta_{\mathrm{MSW}} \equiv \frac{\sin 2 \theta}{\cos 2 \theta-\frac{L_{\mathrm{osc}}}{L_{0}}}
$$

where

$$
L_{0} \equiv \frac{2 \pi}{\sqrt{2} G_{\mathrm{F}} n_{e}}=1.7 \times 10^{7}\left(\frac{\rho}{1 \mathrm{~g} / \mathrm{cm}^{3}}\right)^{-1}\left(\frac{Z}{A}\right)^{-1} \mathrm{~m}
$$

(with the Fermi coupling constant $G_{\mathrm{F}}$ and the electron density $n_{e}$ ) constitutes the path length over which scattering on the electrons changes the electron neutrino phase by $2 \pi$. One can see from Eqs. 2.8 and 2.9 that for $L_{\text {osc }} \gg L_{0}$ the amplitude of the oscillation is suppressed by a factor of $\left(L_{\mathrm{MSW}} / L_{\mathrm{osc}}\right)^{2}$. But Eqs. 2.4 and 2.10 and Table 2.2 imply that inside the Earth this condition is fulfilled for the energies $E$ typically considered in this work $(E \geqslant 100 \mathrm{GeV})$. Hence we may safely ignore the MSW effect. 
The chances of a neutrino actually hitting something as it travels through all this howling emptiness are roughly comparable to that of dropping a ball bearing at random from a cruising 747 and hitting, say, an egg sandwich.

Douglas Adams [84]

\subsection{Neutrino-nucleon interactions}

As neutrinos neither have charge nor partake in strong interactions, they are quite well-known for hardly interacting with other matter. To illustrate the point, let us check the proposition set forth in the introductory quote of this section: The probability of a neutrino interacting on its way through the Galaxy is given by

$$
P_{\text {interaction }}=3 \times 10^{-24}\left(\frac{n_{\text {path }}}{1 \mathrm{~cm}^{-3}}\right)\left(\frac{L_{\text {path }}}{1 \mathrm{kpc}}\right)\left(\frac{\sigma}{10^{-45} \mathrm{~cm}^{2}}\right),
$$

where the indicated value for the neutrino-nucleon cross section $\sigma$ corresponds to an energy of a few hundred keV. [94] On the other hand, the probability of hitting an egg sandwich with a ball bearing thrown from a cruising 747 can (ignoring obstacles such as roofs) roughly be estimated to be

$$
\begin{aligned}
P_{\text {hit sandwich }} \sim 3 & \times 10^{-17}\left(\frac{\text { Earth surface area }}{5.1 \times 10^{14} \mathrm{~m}^{2}}\right)\left(\frac{\text { sandwich area }}{50 \mathrm{~cm}^{2}}\right) \times \\
& \times\left(\frac{\text { sandwich production rate }}{10^{6} \mathrm{yr}^{-1}}\right)\left(\frac{\text { sandwich lifetime }}{100 \mathrm{~s}}\right),
\end{aligned}
$$

so that the probability of a neutrino interaction on the way from, say, the Galactic Center to Earth is even less than that of the ball bearing hitting an egg sandwich. As the column number density along the Earth diameter is about $7 \times 10^{33} \mathrm{~cm}^{-2}$, we can also see from Eq. 2.11 that the probability for some reaction to take place when a neutrino crosses the entire Earth has the value $7 \times 10^{-12}$, which - while being larger than the "sandwich probability" is still an exceedingly small number. Hence the world is fairly transparent for the considered neutrinos.

Things change at higher neutrino energies, though. As we'll see below, the Earth gets opaque for neutrinos at energies above $\gtrsim 100 \mathrm{TeV}$. At such energies, neutrinos and nucleons may undergo either neutral current (NC) 
or charged current interactions (CC). Whereas in the former a neutrino is retained, in the latter the corresponding charged lepton is produced instead, so that for muon neutrinos the two may be written as

$$
\begin{aligned}
& \nu_{\mu}+N \longrightarrow \nu_{\mu}+X(\mathrm{NC}) \\
& \nu_{\mu}+N \longrightarrow \mu+X(\mathrm{CC}),
\end{aligned}
$$

were $X$ denotes some hadronic rest. Equivalent equations hold valid for the other flavors and for antineutrinos. Neutral and charged current interactions proceed via the exchange of $\mathrm{Z}^{0}$ and $\mathrm{W}^{ \pm}$bosons, respectively.

The neutrino-nucleon cross sections are best described in terms of the energy loss

$$
\nu \equiv E_{\text {incoming } \nu}-E_{\text {outgoing lepton }}
$$

and the relative energy loss

$$
y \equiv \frac{E_{\text {incoming } \nu}-E_{\text {outgoing lepton }}}{E_{\text {incoming } \nu}}
$$

in the lab frame, and in terms of the fraction $x$ of the nucleon momentum carried by the interacting parton, given by [140]

$$
x=-Q^{2} / 2 m_{N} \nu
$$

with the nucleon mass $m_{N}$ and

$$
Q^{2} \equiv\left(\boldsymbol{p}_{\text {incoming } \nu}-\boldsymbol{p}_{\text {outgoing lepton }}\right)^{2}-\left(E_{\text {incoming } \nu}-E_{\text {outgoing lepton }}\right)^{2} .
$$

Both $x$ and $y$ may have a value between 0 and 1 . For simplicity, we won't distinguish between the proton and neutron mass.

The actual calculation of the neutrino-nucleon cross sections is somewhat tedious, and its gory details can be found in, e.g., [41, 72]. For charged current interactions, it results in the following formulae:

$$
\begin{array}{r}
\frac{\mathrm{d}^{2} \sigma_{\nu_{\mu} p, \mathrm{CC}}}{\mathrm{d} x \mathrm{~d} y}=\frac{2 G_{\mathrm{F}}^{2} m_{p} E_{\nu}}{\pi}\left(\frac{m_{W}^{2}}{Q^{2}+m_{W}^{2}}\right)^{2} x\left[\left(d\left(x, Q^{2}\right)+s\left(x, Q^{2}\right)+b\left(x, Q^{2}\right)\right)\right. \\
\left.+(1-y)^{2}\left(\bar{u}\left(x, Q^{2}\right)+\bar{c}\left(x, Q^{2}\right)+\bar{t}\left(x, Q^{2}\right)\right)\right] \\
\frac{\mathrm{d}^{2} \sigma_{\bar{\nu}_{\mu} p, \mathrm{CC}}}{\mathrm{d} x \mathrm{~d} y}=\frac{2 G_{\mathrm{F}}^{2} m_{p} E_{\nu}}{\pi}\left(\frac{m_{W}^{2}}{Q^{2}+m_{W}^{2}}\right)^{2} x\left[\left(\bar{d}\left(x, Q^{2}\right)+\bar{s}\left(x, Q^{2}\right)+\bar{b}\left(x, Q^{2}\right)\right)\right. \\
\left.+(1-y)^{2}\left(u\left(x, Q^{2}\right)+c\left(x, Q^{2}\right)+t\left(x, Q^{2}\right)\right)\right]
\end{array}
$$




$$
\begin{array}{r}
\frac{\mathrm{d}^{2} \sigma_{\nu_{\mu} n, \mathrm{CC}}}{\mathrm{d} x \mathrm{~d} y}=\frac{2 G_{\mathrm{F}}^{2} m_{p} E_{\nu}}{\pi}\left(\frac{m_{W}^{2}}{Q^{2}+m_{W}^{2}}\right)^{2} x\left[\left(u\left(x, Q^{2}\right)+s\left(x, Q^{2}\right)+b\left(x, Q^{2}\right)\right)\right. \\
\left.+(1-y)^{2}\left(\bar{d}\left(x, Q^{2}\right)+\bar{c}\left(x, Q^{2}\right)+\bar{t}\left(x, Q^{2}\right)\right)\right] \\
\begin{array}{r}
\frac{\mathrm{d}^{2} \sigma_{\bar{\nu}_{\mu} n, \mathrm{CC}}}{\mathrm{d} x \mathrm{~d} y}=\frac{2 G_{\mathrm{F}}^{2} m_{p} E_{\nu}}{\pi}\left(\frac{m_{W}^{2}}{Q^{2}+m_{W}^{2}}\right)^{2} x\left[\left(\bar{u}\left(x, Q^{2}\right)+\bar{s}\left(x, Q^{2}\right)+\bar{b}\left(x, Q^{2}\right)\right)\right. \\
\left.+(1-y)^{2}\left(d\left(x, Q^{2}\right)+c\left(x, Q^{2}\right)+t\left(x, Q^{2}\right)\right)\right]
\end{array}
\end{array}
$$

In these equations, the functions $u, d, c, s, t$ and $b$ constitute the probability density for encountering a quark of the respective flavor with the fraction $x$ of the nucleon momentum, which is known as the parton distribution function.

Similarly, neutral current interactions are described by:

$$
\begin{aligned}
& \frac{\mathrm{d}^{2} \sigma_{\nu_{\mu} p / n, \mathrm{NC}}}{\mathrm{d} x \mathrm{~d} y}=\frac{G_{\mathrm{F}}^{2} m_{n} E_{\nu}}{\pi}\left(\frac{m_{Z}^{2}}{Q^{2}+m_{Z}^{2}}\right)^{2}\left[A_{L}^{p / n}\left(x, Q^{2}\right)+(1-y)^{2} A_{R}^{p / n}\left(x, Q^{2}\right)\right] \\
& \frac{\mathrm{d}^{2} \sigma_{\bar{\nu}_{\mu} p / n, \mathrm{NC}}}{\mathrm{d} x \mathrm{~d} y}=\frac{G_{\mathrm{F}}^{2} m_{n} E_{\nu}}{\pi}\left(\frac{m_{Z}^{2}}{Q^{2}+m_{Z}^{2}}\right)^{2}\left[A_{R}^{p / n}\left(x, Q^{2}\right)+(1-y)^{2} A_{L}^{p / n}\left(x, Q^{2}\right)\right]
\end{aligned}
$$

Here, $m_{Z}$ denotes the mass of the $\mathrm{Z}$ boson, and the functions $A_{L}^{p / n}, A_{R}^{p / n}$ are defined as

$$
\begin{gathered}
A_{L}^{p}\left(x, Q^{2}\right) \equiv 2 x\left[\epsilon_{L}^{2}(u)(u+c+t)+\epsilon_{L}^{2}(d)(d+s+b)\right. \\
\left.+\epsilon_{R}^{2}(u)(\bar{u}+\bar{c}+\bar{t})+\epsilon_{R}^{2}(d)(\bar{d}+\bar{s}+\bar{b})\right] \\
A_{R}^{p}\left(x, Q^{2}\right) \equiv 2 x\left[\epsilon_{R}^{2}(u)(u+c+t)+\epsilon_{R}^{2}(d)(d+s+b)\right. \\
\left.+\epsilon_{L}^{2}(u)(\bar{u}+\bar{c}+\bar{t})+\epsilon_{L}^{2}(d)(\bar{d}+\bar{s}+\bar{b})\right] \\
A_{L}^{n}\left(x, Q^{2}\right) \equiv 2 x\left[\epsilon_{L}^{2}(u)(d+c+t)+\epsilon_{L}^{2}(d)(u+s+b)\right. \\
\left.+\epsilon_{R}^{2}(u)(\bar{d}+\bar{c}+\bar{t})+\epsilon_{R}^{2}(d)(\bar{u}+\bar{s}+\bar{b})\right] \\
A_{R}^{n}\left(x, Q^{2}\right) \equiv 2 x\left[\epsilon_{R}^{2}(u)(d+c+t)+\epsilon_{R}^{2}(d)(u+s+b)\right. \\
\left.+\epsilon_{L}^{2}(u)(\bar{d}+\bar{c}+\bar{t})+\epsilon_{L}^{2}(d)(\bar{u}+\bar{s}+\bar{b})\right]
\end{gathered}
$$

where we have omitted the arguments for the parton distributions and where

$$
\epsilon_{L}(q)= \begin{cases}+\frac{1}{2}-\frac{2}{3} \sin ^{2} \theta_{\mathrm{W}} & (\mathrm{q}=\mathrm{u}) \\ -\frac{1}{2}+\frac{1}{3} \sin ^{2} \theta_{\mathrm{W}} & (\mathrm{q}=\mathrm{d})\end{cases}
$$


and

$$
\epsilon_{R}(q)= \begin{cases}-\frac{2}{3} \sin ^{2} \theta_{\mathrm{W}} & (\mathrm{q}=\mathrm{u}) \\ +\frac{1}{3} \sin ^{2} \theta_{\mathrm{W}} & (\mathrm{q}=\mathrm{d})\end{cases}
$$

with the Weinberg angle $\theta_{\mathrm{W}}$. If we denote the fractional part of protons in the target medium (i.e. the ratio of the charge number $Z$ and the mass number $A$ ) by $R_{p}$, the charged current neutrino-nucleon cross sections obviously are given by

$$
\frac{\mathrm{d}^{2} \sigma_{\nu_{\mu}, \mathrm{CC}}\left(E_{\nu}, x, y\right)}{\mathrm{d} x \mathrm{~d} y}=R_{p} \frac{\mathrm{d}^{2} \sigma_{\nu_{\mu} p, \mathrm{CC}}\left(E_{\nu}, x, y\right)}{\mathrm{d} x \mathrm{~d} y}+\left(1-R_{p}\right) \frac{\mathrm{d}^{2} \sigma_{\nu_{\mu} n, \mathrm{CC}}\left(E_{\nu}, x, y\right)}{\mathrm{d} x \mathrm{~d} y},
$$

and corresponding relations hold valid for antineutrinos and for the neutral current cross sections. We adopt the usual choice $R_{p}=0.5$ ("isoscalar target"), which is shown to be a reasonable approximation by Table 6.1.

In this work, the CTEQ6DIS parton distributions are used, which are computed by means of the program provided by the Coordinated TheoreticalExperimental Project on QCD [59]. (See Figs. 2.1-2.4 for a comparison between the cross sections and inelasticities for various parton distributions.) An extrapolation to $x$ values below $x_{\min }=10^{-6}$, which aren't covered by this program, would in principle require an elaborate computation such as the solution of the Altarelli-Parisi [13] or Balitskiü-Fadin-Kuraev-Lipatov equations $[75,123,124,32,133]$. However, we simplify the calculation by assuming that the parton distribution functions $q$ may be regarded as being of power-law form below $x_{\text {min }}$,

$$
q(x, Q)=q\left(x_{\min }, Q\right)\left(\frac{x}{x_{\min }}\right)^{\gamma},
$$

where the power law index $\gamma$ is chosen so that the derivative of $q$ remains continuous at $x=x_{\min }$. Similarly, we make the simplifying assumption that for values of $Q$ outside the range $\left[Q_{\min }, Q_{\max }\right]$ covered by the program, the parton distribution functions are given by

$$
q(x, Q)=\left\{\begin{array}{ll}
q\left(x, Q_{\min }\right) & \left(Q<Q_{\min }\right) \\
q\left(x, Q_{\max }\right) & \left(Q>Q_{\max }\right)
\end{array} .\right.
$$

By integrating the formulae for the differential cross sections over $x$ and $y$, one can easily see that for initial neutrino energies $\ll m_{W}, m_{Z}$ in the center 


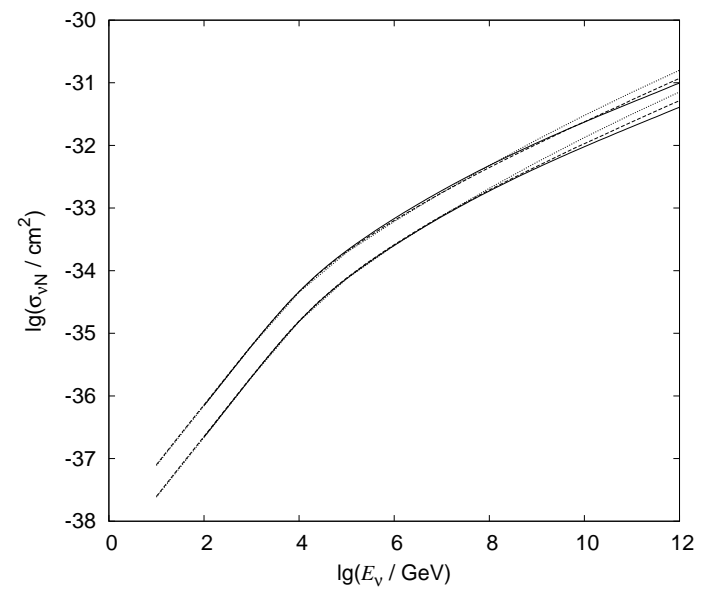

Figure 2.1: Total cross sections for charged current (upper) and neutral current (lower curves) neutrino-nucleon interactions. The cross sections for the CTEQ3DIS [86] (dotted) and CTEQ4DIS parton distribution functions [87] (dashed) as well as the cross sections used in this work (solid lines) are shown.

of mass frame, the neutrino-nucleon cross sections scale linearly with the energy. At energies above the boson mass, however, their slope decreases considerably (cf. Figs. 2.1 and 2.2).

So far, we have considered muon neutrinos only. However, at sufficiently large energies, where the lepton mass doesn't matter any longer, one should expect the cross sections to be independent of the neutrino flavor. Fig. 2.5 illustrates this for the case of charged current cross sections. We thus assume that for electron and tau neutrinos the neutrino-nucleon cross sections equal those for muon neutrinos.

Finally, we note that the diameter of the Earth contains about $6.5 \times 10^{33}$ nucleons per square centimeter and that the total neutrino-nucleon cross section at $100 \mathrm{TeV}$ is of the order of $10^{-34} \mathrm{~cm}^{2}$, so that the product of the two is of the order 1. But this means that the Earth does indeed get opaque at energies above $\gtrsim 100 \mathrm{TeV}$, as claimed above.

\section{$2.3 \quad$ Neutrino-electron interactions}

In addition to the interactions with nucleons covered by the previous section, neutrinos may also interact with electrons. As these have no inner structure, 


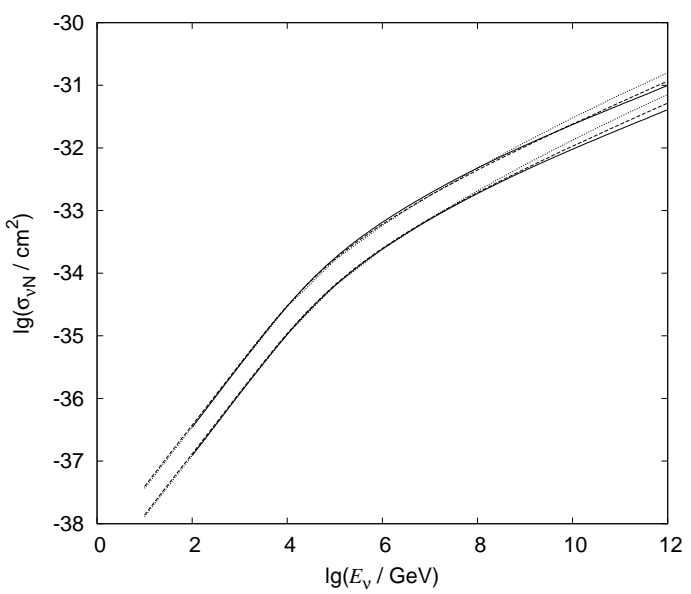

Figure 2.2: Total cross sections for charged current (upper) and neutral current (lower curves) antineutrino-nucleon interactions. The cross sections for the CTEQ3DIS [86] (dotted) and CTEQ4DIS parton distribution functions [87] (dashed) as well as the cross sections used in this work (solid lines) are shown.

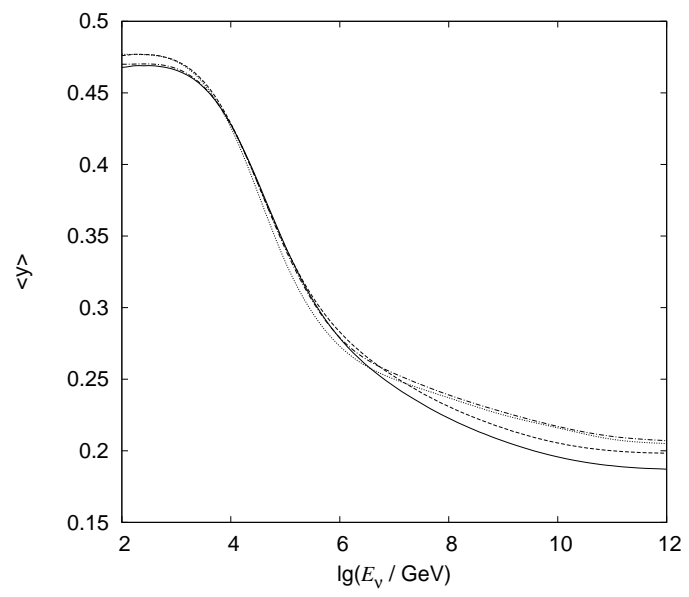

Figure 2.3: Average inelasticity parameter $y$ for charged current neutrino-nucleon interactions with the CTEQ3DIS [86] parton distribution functions (PDFs) (dotted) and the PDFs used in this work (dashed curve), and for neutral current neutrino-nucleon interactions, again with the CTEQ3DIS PDFs (dot-dashed) and the PDFs used in this work (solid curve). 


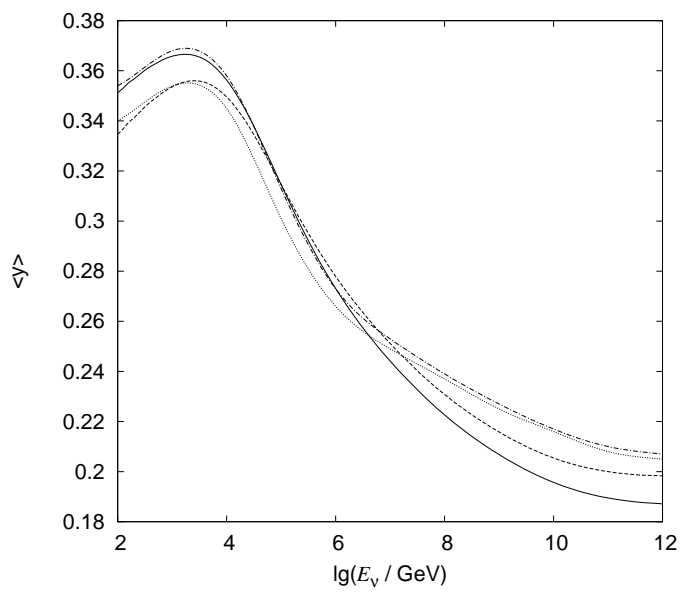

Figure 2.4: Average inelasticity parameter $y$ for charged current antineutrinonucleon interactions with the CTEQ3DIS [86] parton distribution functions (PDFs) (dotted) and the PDFs used in this work (dashed curve), and for neutral current neutrino-nucleon interactions, again with the CTEQ3DIS PDFs (dotdashed) and the PDFs used in this work (solid curve).

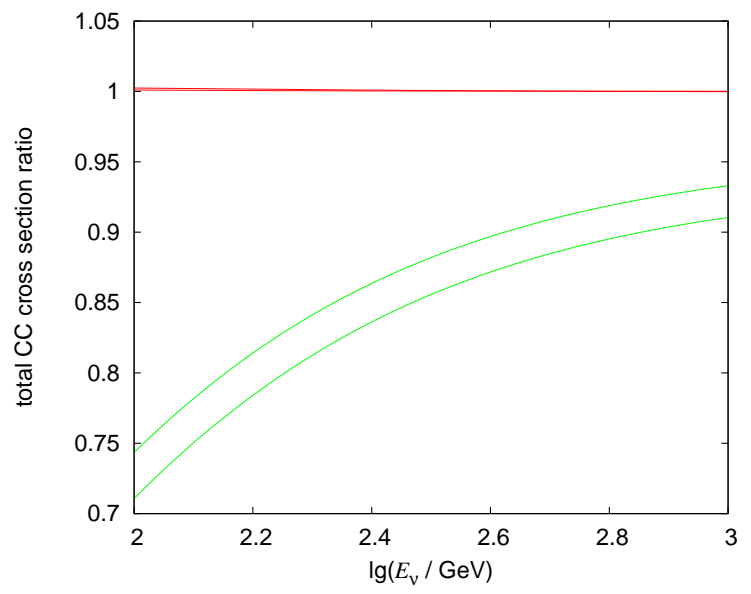

Figure 2.5: Ratio of the total charged current deep inelastic scattering neutrinonucleon cross sections in an isoscalar target for $\nu_{e}$ and $\nu_{\mu}$ (upper (at $100 \mathrm{GeV}$ ) red), $\bar{\nu}_{e}$ and $\bar{\nu}_{\mu}$ (lower red), $\nu_{\tau}$ and $\nu_{\mu}$ (upper green) and $\bar{\nu}_{\tau}$ and $\bar{\nu}_{\mu}$ (lower green curve). The ratios have been obtained from the ICARUS cross section data. [107] 
the corresponding cross section formulae are substantially simpler and can be stated analytically. They are given by [86], and a pedagogical derivation for some of them may be found in [132].

We start with the process $\nu_{e}+e \longrightarrow \nu_{e}+e$. An inspection of the Feynman diagram vertices for weak interactions serves to show that this reaction may take place through the exchange of either a $\mathrm{Z}$ or a $\mathrm{W}$ boson (ignoring Higgs bosons). The corresponding differential cross section has the form

$$
\begin{aligned}
\frac{\mathrm{d} \sigma\left(\nu_{e}+e \longrightarrow\right.}{\mathrm{d} y} & \left.\nu_{e}+e\right) \\
& +\left(\frac { G _ { \mathrm { F } } ^ { 2 } m _ { e } E _ { \nu } } { 2 \pi } \left[\frac{R_{e}^{2}(1-y)^{2}}{\left(1+2 m_{e} E_{\nu} y / m_{Z}^{2}\right)^{2}}\right.\right. \\
& \left.\left.=\frac{2}{1+2 m_{e} E_{\nu} y / m_{Z}^{2}}+\frac{2}{1+2 m_{e} E_{\nu}(1-y) / m_{W}^{2}}\right)^{2}\right]
\end{aligned}
$$

with the chiral couplings $L_{e}=2 \sin ^{2} \theta_{\mathrm{W}}-1$ and $R_{e}=2 \sin ^{2} \theta_{\mathrm{W}}$. The corresponding cross section for electron antineutrinos is given by

$$
\begin{aligned}
& \frac{\mathrm{d} \sigma\left(\bar{\nu}_{e}+e \longrightarrow \bar{\nu}_{e}+e\right)}{\mathrm{d} y}=\frac{G_{\mathrm{F}}^{2} m_{e} E_{\nu}}{2 \pi}\left[\frac{R_{e}^{2}}{\left(1+2 m_{e} E_{\nu} v / m_{Z}^{2}\right)^{2}}\right. \\
& \left.+\left|\frac{L_{e}}{1+2 m_{e} E_{\nu} y / m_{Z}^{2}}+\frac{2}{1-2 m_{e} E_{\nu} / m_{W}^{2}+i \Gamma_{W} / m_{W}}\right|^{2}(1-y)^{2}\right],
\end{aligned}
$$

where $\Gamma_{W}=2.124 \mathrm{GeV}[70]$ is the width of the $\mathrm{W}$ boson. The scattering of electron antineutrinos on electrons may also result in hadron production,

$$
\frac{\mathrm{d} \sigma\left(\bar{\nu}_{e}+e \longrightarrow \text { hadrons }\right)}{\mathrm{d} y}=\frac{\mathrm{d} \sigma\left(\bar{\nu}_{e}+e \longrightarrow \bar{\nu}_{\mu}+\mu\right)}{\mathrm{d} y} \frac{\Gamma(W \longrightarrow \text { hadrons })}{\Gamma\left(W \longrightarrow \bar{\nu}_{\mu}+\mu\right)} .
$$

Here the $\Gamma$ 's denote the relative frequency of the given process. As muon neutrinos cannot be transformed into electrons via a $\mathrm{W}$ boson, it is clear that the process $\nu_{\mu}+e \longrightarrow \nu_{\mu}+e$ can proceed via $\mathrm{Z}$ boson exchange only. Hence its cross section ought to differ from that of $\nu_{e} e$ scattering, and indeed it is obtained from the latter by dropping the terms due to $\mathrm{W}$ boson exchange:

$$
\frac{\mathrm{d} \sigma\left(\nu_{\mu}+e \longrightarrow \nu_{\mu}+e\right)}{\mathrm{d} y}=\frac{G_{\mathrm{F}}^{2} m_{e} E_{\nu}}{2 \pi} \frac{1}{\left(1+2 m_{e} E_{\nu} y / m_{Z}^{2}\right)^{2}}\left[R_{e}^{2}(1-y)^{2}+L_{e}^{2}\right]
$$

Similarly, the corresponding cross section for muon antineutrinos has the form

$$
\frac{\mathrm{d} \sigma\left(\bar{\nu}_{\mu}+e \longrightarrow \bar{\nu}_{\mu}+e\right)}{\mathrm{d} y}=\frac{G_{\mathrm{F}}^{2} m_{e} E_{\nu}}{2 \pi} \frac{1}{\left(1+2 m_{e} E_{\nu} y / m_{Z}^{2}\right)^{2}}\left[R_{e}^{2}+L_{e}^{2}(1-y)^{2}\right]
$$


While it is true that a $\mathrm{W}$ boson exchange cannot turn a muon neutrino into an electron, it may well turn it into a muon, so that the process $\nu_{\mu}+e \longrightarrow \mu+\nu_{e}$ is possible. It has the cross section

$$
\frac{\mathrm{d} \sigma\left(\nu_{\mu}+e \longrightarrow \mu+\nu_{e}\right)}{\mathrm{d} y}=\frac{G_{\mathrm{F}}^{2} m_{e} E_{\nu}}{2 \pi} \frac{4\left[1-\left(m_{\mu}^{2}-m_{e}^{2}\right) / 2 m_{e} E_{\nu}\right]^{2}}{\left(1+2 m_{e} E_{\nu}(1-y) / m_{W}^{2}\right)^{2}} .
$$

Obviously, the cross section for tau (anti)neutrino-electron scattering is obtained from that for muon (anti)neutrinos by replacing the muon with the tauon mass.

If one compares the cross sections for neutrino-electron scattering with those for neutrino-nucleon scattering, one reaches the conclusion that the former exceed the latter in a very small range around the $\mathrm{W}$ boson resonance only. For this reason, we neglect them in this work.

\subsection{Lepton energy losses and decay}

Laboratory experiments indicate that the lifetime of electrons exeeds $4.6 \times$ $10^{26}$ years [70], so that their overall number doesn't change due to decay. When moving through matter, however, they suffer from energy losses, and hence it makes sense to speak of a mean path length $L$. This can be approximated by the formula [86]

$$
L(E) \approx 40\left(\frac{\rho}{1 \mathrm{~g} / \mathrm{cm}^{3}}\right)^{-1}\left(\frac{E}{62 \mathrm{TeV}}\right)^{1 / 2} \mathrm{~cm},
$$

which takes the Landau-Pomeranchuk-Migdal (LPM) effect [146, 22] into account. Muons, on the other hand, are unstable and may decay by either of the processes [93]

$$
\begin{array}{ll}
\mu^{-} \longrightarrow e^{-}+\bar{\nu}_{e}+\nu_{\mu} & (f \approx 1) \\
\mu^{-} \longrightarrow e^{-}+\bar{\nu}_{e}+\nu_{\mu}+\gamma & (f \sim 0.01) \\
\mu^{-} \longrightarrow e^{-}+\bar{\nu}_{e}+\nu_{\mu}+e^{-}+e^{+} & \left(f \sim 10^{-7}\right)
\end{array}
$$

where the branching ratio $f$ is defined as the relative fraction with which a given mode occurs in the decay. The mean lifetime of a muon at rest has the value $\tau_{\mu}=2.197 \times 10^{-6} \mathrm{~s}[70]$. For a moving muon, however, due to 
time dilation, the lifetime in the laboratory frame is enlarged by its $\gamma$ factor. Accordingly, the number $N_{\mu}$ of muons with some given energy must fulfill the relation

$$
\frac{d N_{\mu}(E, t)}{\mathrm{d} t}=-\frac{1}{\gamma \tau_{\mu}} N(E, t)=-\frac{1}{\tau_{\mu}} \frac{m_{\mu} c^{2}}{E} N(E, t) .
$$

If the muon energy was constant, the solution to this equation would simply be

$$
N(E, t)=N(E, t=0) \exp \left(-\frac{m_{\mu} c^{2}}{\tau_{\mu} E}\right) .
$$

But, alas, the muon energy is anything but constant. Indeed, ionization, pair production, bremsstrahlung, and photonuclear interactions lead to energy losses that may roughly be piecewise parametrized by the formula

$$
\frac{\mathrm{d} E}{\mathrm{~d} X}=-(\alpha+\beta E)
$$

the solution of which has the form

$$
X=\frac{1}{\beta} \ln \frac{E(X=0)+\frac{\alpha}{\beta}}{E(X)+\frac{\alpha}{\beta}}
$$

The values of $\alpha$, which covers the ionization, and $\beta$, which covers the other loss proccesses, depend on the energy, yielding [118]

$$
\frac{\mathrm{d} E_{\mu}(E)}{\mathrm{d} X}=\left\{\begin{array}{r}
2.30 \times 10^{-3} \mathrm{GeV} \mathrm{cm}^{2} \mathrm{~g}^{-1}+15.50 \times 10^{-6} \mathrm{~cm}^{2} \mathrm{~g}^{-1}(E / 1 \mathrm{GeV}) \\
(E \leqslant 30.0 \mathrm{GeV}) \\
2.67 \times 10^{-3} \mathrm{GeV} \mathrm{cm}^{2} \mathrm{~g}^{-1}+3.40 \times 10^{-6} \mathrm{~cm}^{2} \mathrm{~g}^{-1}(E / 1 \mathrm{GeV}) \\
(30.0 \mathrm{GeV}<E \leqslant 35.3 \mathrm{TeV}) \\
-6.50 \times 10^{-3} \mathrm{GeV} \mathrm{cm}^{2} \mathrm{~g}^{-1}+3.66 \times 10^{-6} \mathrm{~cm}^{2} \mathrm{~g}^{-1}(E / 1 \mathrm{GeV}) \\
(35.3 \mathrm{TeV}<E)
\end{array}\right.
$$

Note that Eqs. 2.13 and 2.15 make the implicit assumption that the muon energy losses are continuous. There are, however, fluctuations, and a more accurate calculation should pay heed to this fact, as described in [118]. The influence of the LPM and Ter-Mikaelian effect is discussed in [163]. 
In order to estimate the importance of the energy losses, we consider the fraction $r$ of muons with an initial energy $E_{0} \equiv E(X=0)$ which reach the energy $a E_{0}$ (with $a<1$ ) before decaying. From Eq. 2.14 and $X=\rho c t$ we know that such muons must exist at least for a time

$$
t_{a}=\frac{1}{\beta \rho c} \ln \frac{E_{0}+\frac{\alpha}{\beta}}{a E_{0}+\frac{\alpha}{\beta}} .
$$

As the muon lifetime increases with energy, a lower limit for the fraction $r$ can be obtained by inserting the minimum survival time $t_{a}$ and the final energy $a E_{0}$ into Eq. 2.12,

$r=\frac{N_{\mu}\left(t=t_{a}\right)}{N_{\mu}(t=0)}>\exp \left(-\frac{m_{\mu} c^{2}}{\tau_{\mu} a E_{0}} \cdot \frac{1}{\beta \rho c} \ln \frac{E_{0}+\frac{\alpha}{\beta}}{a E_{0}+\frac{\alpha}{\beta}}\right)=\left(\frac{a E_{0}+\frac{\alpha}{\beta}}{E_{0}+\frac{\alpha}{\beta}}\right)^{\frac{1}{\beta \rho c} \frac{m_{\mu} c^{2}}{a E_{0}} \frac{1}{\tau_{\mu}}}$.

As an example, we note that for $a=0.01, E_{0}=10^{6} \mathrm{GeV}\left(100 \mathrm{GeV}, 10^{10} \mathrm{GeV}\right)$ and $\rho=4 \mathrm{~g} / \mathrm{cm}^{3}, r$ is greater than $0.99995\left(0.987,1.0-5 \times 10^{-9}\right)$, so that the vast majority of muons decay at an energy less than $1 \%$ of the initial value. Hence it makes sense to say that muons loose all their energy prior to their decay.

This is not true for tauons. As these have a substantially larger mass, they decay more rapidly, their mean lifetime being only $2.91 \times 10^{-13} \mathrm{~s}$ [70]. As in the case of muons, the energy losses are due to ionization, pair production, bremsstrahlung, and photonuclear interactions, and again they may be approximated by an equation of the form of Eq. 2.13. Details can be found in $[66,67]$. Essentially, tauon energy losses become important only at energies $\gtrsim 10^{8} \mathrm{GeV}$.

Hence it is necessary to discuss tauon decay in more detail. This can be achieved by noting that for a tauon moving nearly with the speed of light, the probability that the tauon decays in a given mode $\mathrm{D}$ and thus produces a particle $B$ with the energy $z E_{\tau}$ can be written as

$$
\frac{\mathrm{d} P^{(\mathrm{D}, B)}(z)}{\mathrm{d} z}=f^{(\mathrm{D})} \frac{\mathrm{d} \Gamma^{(\mathrm{D}, B)}(z)}{\mathrm{d} z},
$$

where $f^{(D)}$ is the branching ratio of $D$ and where $\mathrm{d} \Gamma^{(\mathrm{D}, B)} / \mathrm{d} z$ denotes the normalized spectrum of the resulting particle $B$ (i.e. $\int_{0}^{1} \mathrm{~d} z \mathrm{~d} \Gamma^{\mathrm{D}, B} / \mathrm{d} z=1$ ). Therefore, the differential production rate of $B$ from the decay mode $\mathrm{D}$ is 


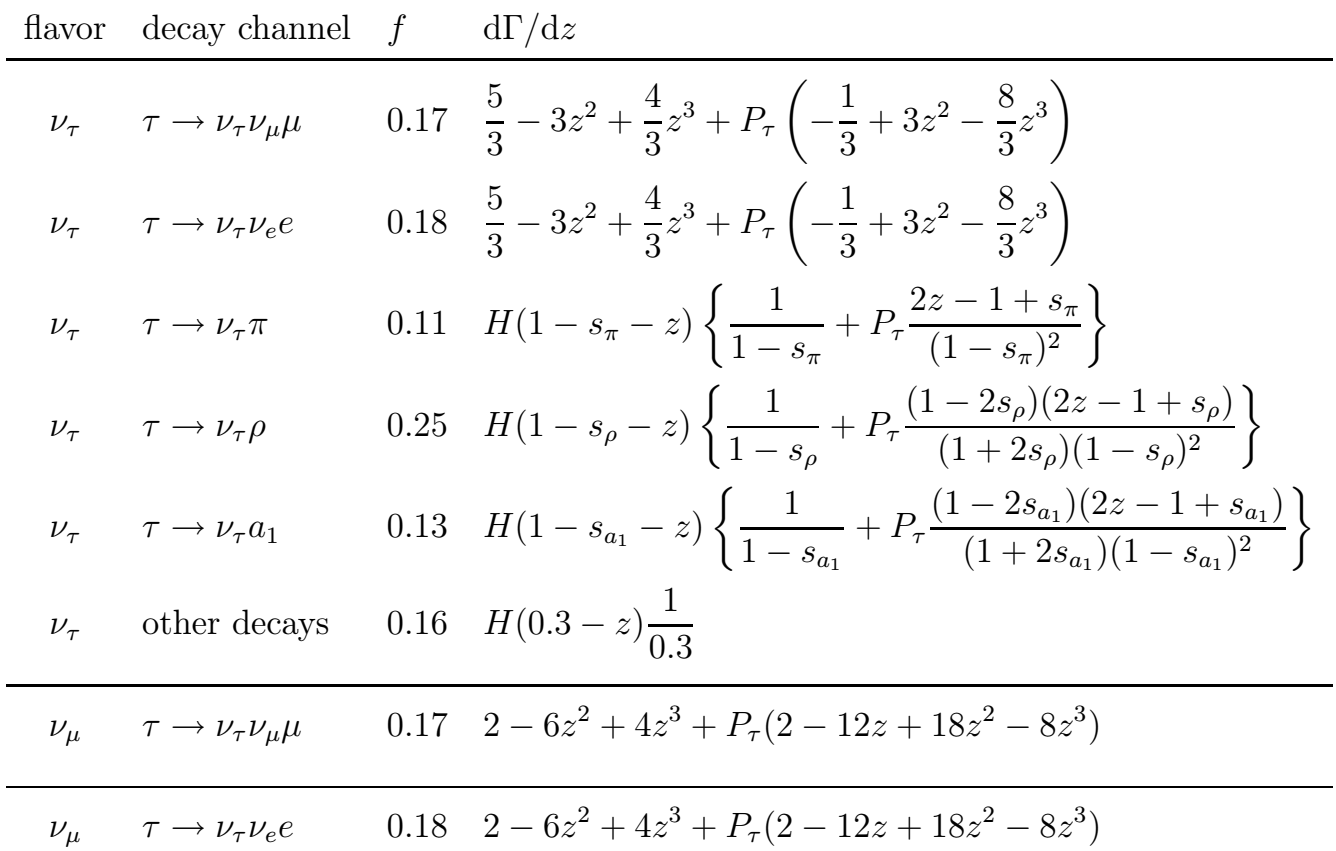

Table 2.3: Branching ratio $f[161,68,93]$ and normalized neutrino production spectra $\mathrm{d} \Gamma / \mathrm{d} z[161,68,83]$ in the limit $\beta_{\tau} \longrightarrow 1$ for the tauon decay modes. $P_{\tau}$ denotes the tauon polarization, $H$ the Heaviside function, and $z$ the ratio $E_{\nu} / E_{\tau}$ of the neutrino and the initial tauon energy. $s_{i}$ is defined as $m_{i}^{2} / m_{\tau}^{2}$. The spectrum given for the "other decays" is an approximation.

given by

$$
\frac{\mathrm{d}^{2} N^{(\mathrm{D}, B)}\left(E_{\tau}, z\right)}{\mathrm{d} z \mathrm{~d} t}=\frac{f^{(\mathrm{D})}}{\tau_{\tau}} \frac{m_{\tau} c^{2}}{E_{\tau}} \frac{\mathrm{d} \Gamma^{(\mathrm{D}, B)}(z)}{\mathrm{d} z} N_{\tau}\left(E_{\tau}\right) .
$$

Table 2.3 contains the branching ratios and normalized decay spectra for various tauon decay modes. Pedagogical illustrations of how to obtain decay spectra in the lepton rest frame and how to transform them to the laboratory frame can be found in $[158,83]$. 


\section{Chapter 3}

\section{Propagation analysis}

As was shown at the end of Sect. 2.2 , for neutrino energies exceeding $10^{5} \mathrm{GeV}$ the Earth starts to become opaque, so that for sufficiently large energies, a propagation analysis is warranted. After deriving the equations for this analysis, we discuss three methods for their solution, namely integral transformations (Sect. 3.2), discretization (Sect. 3.3), and iteration (Sect. 3.4). Of course, Monte Carlo simulations can be employed as well. Respective discussions can be found in, e.g., [34, 154, 49, 67].

\subsection{The cascade equations}

Before developing the cascade equations for the description of neutrino propagation, it is instructive to outline the basic ideas qualitatively first. Obviously everything begins with a neutrino entering some medium, where it may interact with a nucleon, even though the probability for this is extremely small. If such an interaction happens to be a neutral current scattering, the neutrino persists to exist. If, however, the scattering is a charged current one, the neutrino is turned into the corresponding charged lepton.

In case of an electron or muon neutrino the latter means from a practical point of view that the neutrino is lost for good, as electrons are stable and muons radiate their energy away before decaying back into a muon (and electron) neutrino (cf. Sect. 2.4). To be slightly more precise, one should argue that the energies of neutrinos from muon decays are so low compared to the values of the original neutrinos that, as the neutrino flux decreases with energy, their flux doesn't contribute significantly. 


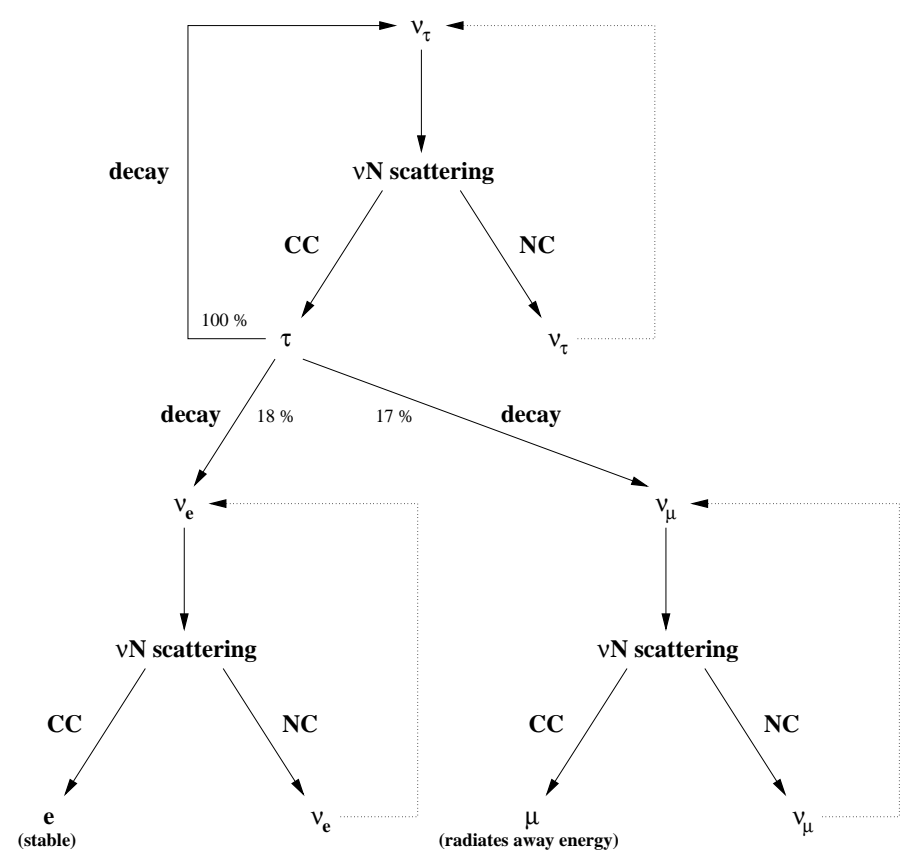

Figure 3.1: The general concepts involved in the neutrino propagation through matter. Neutrino-electron interactions, which are not included in the figure, can be treated completely analogically. See the main text for an explanation.

In case of a tauon, on the other hand, the decay leads to a neutrino which still has to be taken into account. In other words, in a charged current tau neutrino-nucleon interaction the tau neutrino is regenerated. About $20 \%$ of the tauon decays also result in the creation of an electron neutrino, and the same is true for muon neutrinos (cf. the branching ratios in Table 2.3).

Hence with regard to neutrino-nucleon interactions, the number of tau neutrinos remains constant throughout the propagation, whereas that of electron and muon neutrinos decreases. But even if the neutrino is preserved in a scattering process, one should bear in mind that its energy is always diminished.

Further changes in the neutrino numbers are inflicted by neutrino-electron scatterings. These are far smaller, however, and can usually be neglected without loosing too much accuracy. Fig. 3.1 illustrates the relevant concepts for the neutrino propagation.

Now in general, the description of a flux of particles traversing some medium qualitatively boils down to the simple statement that the flux at 
some given energy will be diminished by scattering, decay, radiative losses and the like, but will at the same time be enhanced by the same processes taking place at higher energies.

In principle this constitutes a three-dimensional problem, as scattered particles might leave in any direction. Fortunately enough, though, the propagation of high energy neutrinos is essentially a one-dimensional one. To see why, let us first consider the neutrino-nucleon scattering

$$
\nu_{l}+N \longrightarrow L+X,
$$

where $L$ may be either a neutrino $\nu_{l}$ or the corresponding charged lepton $l$. Using the conservation of (relativistic) energy and momentum, one may show that the angle $\theta$ between the momenta $\boldsymbol{p}$ and $\boldsymbol{p}^{\prime}$ of $\nu_{l}$ and $L$ is given by

$$
\cos \theta=\frac{1}{2\left|\boldsymbol{p}^{\prime}\right||\boldsymbol{p}|}\left(m_{X}^{2}-m_{N}^{2}-m_{\nu_{l}}^{2}-m_{L}^{2}+\frac{2 E_{\nu_{l}} E_{L}}{c^{2}}-2 m_{N}\left(E_{\nu_{l}}-E_{l}\right)\right) .
$$

But the energies we are interested in are much larger than the rest energies of the various particles involved, which implies that $|\boldsymbol{p}|=E_{\nu_{l}} / c$ and $\left|\boldsymbol{p}^{\prime}\right|=$ $E_{L} / c$. Hence we obtain $\cos \theta=1$, and thus $L$ must have the same direction as the incoming neutrino.

Similar arguments apply to the decay of a lepton. For an order of magnitude analysis we may take the decay products to be distributed isotropically in the lepton rest frame. Then, as the considered tauons move nearly with the speed of light, the mean angle $\theta$ between their momentum and the momenta of the outgoing particles is of the order of $1 / \gamma=m_{\tau} c^{2} / E_{\tau} \ll 1$ in the laboratory frame (cf. Eq. (4.10) of [174]). Hence the decay products move in the same direction as the original tauon.

The case of neutrino-electron scatterings could be treated analogically. Thus all the particles considered in a neutrino propagation analysis move in the same direction, and the problem contains one dimension only. In the following we denote the distance along the linear path of the particles by $z$.

We may now put the ideas presented so far in more quantitative terms. To do so, let us consider the fluxes $\phi_{i}$ of $M$ different particles. Then differential transfer functions $\mathrm{d}^{2} T_{i k}^{(I)} / \mathrm{d} E \mathrm{~d} z$ may be introduced by defining that the flux change at energy $E_{\mathrm{f}}$ of particle sort $k$ due to an interaction $I$ of the particle sort $i$ at energy $E_{\mathrm{i}}$ over a differential distance $\mathrm{d} z$ has the value

$$
\mathrm{d} \phi_{k}\left(E_{\mathrm{f}}, z\right)=\frac{\mathrm{d}^{2} T_{i k}^{(I)}\left(E_{\mathrm{i}}, E_{\mathrm{f}}, z\right)}{\mathrm{d} E_{\mathrm{f}} \mathrm{d} z} \phi_{i}\left(E_{\mathrm{i}}, z\right) \mathrm{d} E_{\mathrm{i}} \mathrm{d} z .
$$


Using these functions, the cascade equations describing the particle propagation have the form

$$
\begin{aligned}
\frac{\partial \phi_{k}(E, z)}{\partial z}=-\left(\sum_{i=1}^{M} \sum_{I} \frac{\mathrm{d} T_{k i}^{(I)}(E, z)}{\mathrm{d} z}\right) \phi_{k}(E, z) \\
+\sum_{i=1}^{M} \sum_{I} \int_{E}^{\infty} \mathrm{d} E^{\prime} \frac{\mathrm{d}^{2} T_{i k}^{(I)}\left(E^{\prime}, E, z\right)}{\mathrm{d} E \mathrm{~d} z} \phi_{i}\left(E^{\prime}, z\right),
\end{aligned}
$$

where the sums run over all particle types and all possible interactions $I$, and where $\mathrm{d} T_{k i}^{(I)} / \mathrm{d} z$ denotes the function

$$
\frac{\mathrm{d} T_{k i}^{(I)}(E, z)}{\mathrm{d} z} \equiv \int_{0}^{E} \mathrm{~d} E^{\prime} \frac{\mathrm{d}^{2} T_{k i}^{(I)}\left(E, E^{\prime}, x\right)}{\mathrm{d} E \mathrm{~d} z}
$$

The first term on the right hand side of Eq. 3.2 clearly describes all the losses, whereas the second term gives the gains from interactions at higher energies. In terms of the inelasticity parameter $y=1-E / E^{\prime}$ the differential $\mathrm{d} E$ in the denominator of the transfer function may formally be written as $\mathrm{d} E=E^{\prime} \mathrm{d} y$, and the differential $\mathrm{d} E^{\prime}$ can be viewed as $\mathrm{d} E^{\prime}=\left(E^{\prime 2} / E\right) \mathrm{d} y$. Hence Eq. 3.2 is equivalent to

$$
\begin{aligned}
\frac{\partial \phi_{k}(E, z)}{\partial z}=- & \left(\sum_{i=1}^{M} \sum_{I} \frac{\mathrm{d} T_{k i}^{(I)}(E, z)}{\mathrm{d} z}\right) \phi_{k}(E, z) \\
& +\sum_{i=1}^{M} \int_{0}^{1} \frac{\mathrm{d} y}{1-y} \frac{\mathrm{d}^{2} T_{i k}^{(I)}(E /(1-y), y, z)}{\mathrm{d} y \mathrm{~d} z} \phi_{i}\left(\frac{E}{1-y}, z\right) \mathrm{d} z .
\end{aligned}
$$

It is often useful to discuss the propagation in terms of the (nucleon) column number density $t$,

$$
t(z) \equiv \int_{0}^{z} n_{\text {nucleon }}\left(z^{\prime}\right) \mathrm{d} z^{\prime}=\int_{0}^{z} \frac{\rho\left(z^{\prime}\right)}{m_{p}} \mathrm{~d} z^{\prime} .
$$

The small difference between the proton and neutron mass is neglected in this definition. Using this column number density, the cascade equations can 
be written as

$$
\begin{aligned}
\frac{\partial \phi_{k}(E, t)}{\partial t}=-\frac{m_{p}}{\rho(z)} & \left(\sum_{i=1}^{M} \sum_{I} \frac{\mathrm{d} T_{k i}(E, z)}{\mathrm{d} z}\right) \phi_{k}(E, z) \\
& +\frac{m_{p}}{\rho(z)} \sum_{i=1}^{M} \int_{0}^{1} \frac{\mathrm{d} y}{1-y} \frac{\mathrm{d}^{2} T_{i k}(E /(1-y), y, z)}{\mathrm{d} y \mathrm{~d} z} \phi_{i}\left(\frac{E}{1-y}, z\right) \mathrm{d} z .
\end{aligned}
$$

Here $z$ is to be understood as a function of the column number density, $z=z(t)$. Now the change in the flux of a charged lepton $l$ with an energy of $E_{\mathrm{f}}$ due to charged-current scatterings of the corresponding neutrinos $\nu_{l}$ with an energy of $E_{\mathrm{i}}$ can be expressed as

$$
\mathrm{d} \phi_{l}^{(\mathrm{CC})}(E, z)=n_{N}(z) \frac{\mathrm{d} \sigma_{\mathrm{CC}}\left(E_{\mathrm{i}}, E_{\mathrm{f}}\right)}{\mathrm{d} E} \mathrm{~d} E_{\mathrm{i}} \mathrm{d} z=\frac{\rho(z)}{m_{p}} \frac{\mathrm{d} \sigma_{\mathrm{CC}}\left(E_{\mathrm{i}}, E_{\mathrm{f}}\right)}{\mathrm{d} E} \mathrm{~d} E_{\mathrm{i}} \mathrm{d} z
$$

with the nucleon density $n_{N}$. Comparing this equation to Eq. 3.1 shows immediately that the corresponding transfer function must be

$$
\frac{\mathrm{d}^{2} T_{\nu_{l} l}^{(\mathrm{CC})}\left(E_{\mathrm{i}}, E_{\mathrm{f}}, z\right)}{\mathrm{d} E_{\mathrm{f}} \mathrm{d} z}=\frac{\rho(z)}{m_{p}} \frac{\mathrm{d} \sigma_{\mathrm{CC}}\left(E_{\mathrm{i}}, E_{\mathrm{i}}\right)}{\mathrm{d} E},
$$

and completely analogously we obtain the transfer function

$$
\frac{\mathrm{d}^{2} T_{\nu_{l} \nu_{l}}^{(\mathrm{NC})}\left(E_{\mathrm{i}}, E_{\mathrm{f}}, z\right)}{\mathrm{d} E_{\mathrm{f}} \mathrm{d} z}=\frac{\rho(z)}{m_{p}} \frac{\mathrm{d} \sigma_{\mathrm{NC}}\left(E_{\mathrm{i}}, E_{\mathrm{f}}\right)}{\mathrm{d} E}
$$

for neutral current neutrino-nucleon interactions. As the rate of neutrinoelectron scatterings obviously is proportional to the electron rather than proton density, it is clear that the respective transfer functions are given by

$$
\frac{\mathrm{d}^{2} T^{(\mathrm{S})}\left(E_{\mathrm{i}}, E_{\mathrm{f}}, z\right)}{\mathrm{d} E_{\mathrm{f}} \mathrm{d} z}=n_{e}(z) \frac{\mathrm{d} \sigma^{(\mathrm{S})}\left(E_{\mathrm{i}}, E_{\mathrm{f}}\right)}{\mathrm{d} E}=\frac{\langle Z\rangle}{\langle A\rangle} \frac{\rho(z)}{m_{p}} \frac{\mathrm{d} \sigma^{(\mathrm{S})}\left(E_{\mathrm{i}}, E_{\mathrm{f}}\right)}{\mathrm{d} E},
$$

where the mean atomic number $\langle Z\rangle$ and mean mass number $\langle A\rangle$ may depend on the location $z$. "S" stands for any of the scattering reactions covered by Sect. 2.3. 
As electrons and muons don't contribute to the neutrino flux, all their transfer functions can be disregarded. On the other hand, the decay of tauons must be taken into account, and the contribution of a particular decay channel D for tauons at an energy $E_{\mathrm{i}}$ to the flux $\phi_{k}$ can be written as (cf. Eq. 2.16)

$$
\mathrm{d} \phi_{k}^{(D)}\left(E_{\mathrm{f}}, t\right)=\frac{f^{(D)}}{\tau_{\tau}} \frac{m_{\tau} c^{2}}{E_{\mathrm{i}}} \frac{\mathrm{d} \Gamma^{(\mathrm{D}, \mathrm{k})}\left(E_{\mathrm{i}}, E_{\mathrm{f}}\right)}{\mathrm{d} E} \phi_{\tau}\left(E_{\mathrm{i}}, z\right) \mathrm{d} E_{\mathrm{i}} \mathrm{d} t
$$

where $t$ means the time (rather than the column number density), where $\tau_{\tau}$ is the tauon lifetime at rest, where the factor $m_{\tau} c^{2} / E_{\tau}$ arises from the relativistic time dilation, and where $\mathrm{d} \Gamma^{(\mathrm{D}, \mathrm{k})} / \mathrm{d} E$ denotes the normalized neutrino spectrum for the considered decay channel D, as defined in Sect. 2.4. As we may take the tauons to move at the (vacuum) speed of light, we have $\mathrm{d} z=c \mathrm{~d} t$, so that summing up over all decay modes, we get the transfer function

$$
\frac{\mathrm{d}^{2} T_{i k}^{(\text {decay })}\left(E_{\mathrm{i}}, E_{\mathrm{f}}, z\right)}{\mathrm{d} E_{\mathrm{f}} \mathrm{d} z}=\sum_{D} \frac{f^{(D)}}{c \tau_{\tau}} \frac{m c^{2}}{E_{\mathrm{i}}} \frac{\mathrm{d} \Gamma^{(\mathrm{D}, \mathrm{k})}\left(E_{\mathrm{i}}, E_{\mathrm{f}}\right)}{\mathrm{d} E}
$$

for the tauon decay. As stated earlier, we may neglect neutrino-electron scattering. Hence the cascade equations describing the propagation are obtained by inserting Eqs. 3.6, 3.7, and 3.9 into Eq. 3.4.

At ultrahigh energies, radiative energy losses must be taken into account, and in addition, charged current tauon-nucleon scattering becomes possible. [76] If necessary, these processes can be included in the cascade equations by means of transfer functions the form of which is identical to that of Eq. 3.5 .

\subsection{Integral transformations}

\subsubsection{Formal approach}

In [156], it is shown that for the muon neutrino cascade equation, a formal analytical solution can be found by means of integral transformations, if some simplifying approximations concerning the neutrino-nucleon cross sections are made. Here, we show how to generalize this approach to the case of tau neutrinos and tauons. 
To this end, let us assume (in accordance with [156]) that the differential cross sections as a function of the energy $E$ and the inelasticity parameter $y$ can be written in product form,

$$
\begin{aligned}
& \frac{\mathrm{d} \sigma_{\mathrm{NC}}(E, y)}{\mathrm{d} y}=f(y) \sigma_{\mathrm{NC}}(E) \\
& \frac{\mathrm{d} \sigma_{\mathrm{CC}}(E, y)}{\mathrm{d} y}=g(y) \sigma_{\mathrm{CC}}(E),
\end{aligned}
$$

and that the total cross sections are of power law form,

$$
\sigma_{\mathrm{NC} / \mathrm{CC}}(E)=a_{\mathrm{NC} / \mathrm{CC}} E^{\beta_{\mathrm{NC} / \mathrm{CC}}} .
$$

When writing down the cascade equations in terms of the column number density $t$ (Eq. 3.3), the tauon decay may formally be regarded as a scattering with a $t$-dependent cross section, which can be written as

$$
\frac{\mathrm{d} \sigma_{\mathrm{dec}}(E, y, t)}{\mathrm{d} y}=\frac{1}{n_{\text {target }}(t)} \frac{\mathrm{d} \sigma_{\mathrm{dec}}(E, y)}{\mathrm{d} y} \equiv P(t) \frac{\mathrm{d} \sigma_{\mathrm{dec}}(E, y)}{\mathrm{d} y}
$$

where the "differential cross section" $\mathrm{d} \sigma_{\mathrm{dec}} / \mathrm{d} y$ must be of the form

$$
\frac{\mathrm{d} \sigma_{\mathrm{dec}}(E, y)}{\mathrm{d} y}=b_{\mathrm{dec}} E^{\kappa} h(y)
$$

with $\kappa=-1$, as the form of a decay spectrum is independent of the initial particle energy and as, due to the relativistic time dilation, the tauon lifetime is proportional to the energy. We may assume without loss of generality that $\int_{0}^{1} h(y) \mathrm{d} y=1$.

Finally, we assume that the radiative tauon energy losses can be described by means of an energy-independent cross section

$$
\frac{\mathrm{d} \sigma_{\mathrm{rad}}(E, y)}{\mathrm{d} y}=\xi_{0}(y)
$$

which corresponds to full screening of the target nucleons (cf. [173]), and that the cross sections for neutral current and charged current tauon-nucleon interactions are given by

$$
\begin{aligned}
& \frac{\mathrm{d} \sigma_{\tau, \mathrm{NC}}(E, y)}{d y}=\sigma_{\tau, \mathrm{NC}}(E) r(y)=b_{\mathrm{NC}} E^{\alpha_{\mathrm{NC}}} r(y) \\
& \frac{\mathrm{d} \sigma_{\tau, \mathrm{CC}}(E, y)}{d y}=\sigma_{\tau, \mathrm{CC}}(E) s(y)=b_{\mathrm{CC}} E^{\alpha_{\mathrm{CC}}} s(y)
\end{aligned}
$$


In order to keep the notation simple, we shall in the following denote the tau neutrino flux with $\phi$ and the tauon flux with $\pi$. Using the formalism of the previous section, we thus see that the cascade equation for the tau neutrinos is given by

$$
\begin{aligned}
\frac{\partial \phi(E, t)}{\partial t}=-( & \left.a_{\mathrm{NC}} E^{\beta_{N C}}+a_{\mathrm{CC}} E^{\beta_{\mathrm{CC}}}\right) \phi(E, t) \\
& +\int_{0}^{1} \frac{\mathrm{d} y}{1-y} a_{\mathrm{NC}}\left(\frac{E}{1-y}\right)^{\beta_{N C}} f(y) \phi\left(\frac{E}{1-y}, t\right) \\
& +\int_{0}^{1} \frac{\mathrm{d} y}{1-y} b_{\mathrm{CC}}\left(\frac{E}{1-y}\right)^{\alpha_{\mathrm{CC}}} s(y) \pi\left(\frac{E}{1-y}, t\right) \\
& +\int_{0}^{1} \frac{\mathrm{d} y}{1-y} P(t) b_{\operatorname{dec}}\left(\frac{E}{1-y}\right)^{\kappa} h(y) \pi\left(\frac{E}{1-y}, t\right),
\end{aligned}
$$

whereas that for the tauons has the form

$$
\begin{aligned}
\frac{\partial \pi(E, t)}{\partial t}=-( & \left.P(t) b_{\mathrm{dec}} E^{\kappa}+\chi+b_{\mathrm{NC}} E^{\alpha_{\mathrm{NC}}}+b_{\mathrm{CC}} E^{\alpha_{\mathrm{CC}}}\right) \pi(E, t) \\
& +\int_{0}^{1} \frac{\mathrm{d} y}{1-y} a_{\mathrm{CC}}\left(\frac{E}{1-y}\right)^{\beta_{\mathrm{CC}}} g(y) \phi\left(\frac{E}{1-y}, t\right) \\
& +\int_{0}^{1} \frac{\mathrm{d} y}{1-y} \xi_{0}(y) \pi\left(\frac{E}{1-y}, t\right) \\
& +\int_{0}^{1} \frac{\mathrm{d} y}{1-y} b_{\mathrm{NC}}\left(\frac{E}{1-y}\right)^{\alpha_{\mathrm{NC}}} r(y) \pi\left(\frac{E}{1-y}, t\right)
\end{aligned}
$$

where $\chi \equiv$ const denotes the total radiative cross section. The first step towards simplifying these equations is to apply the Mellin transform $\mathcal{M}$ [150],

$$
\mathcal{M}[f](s) \equiv \int_{0}^{\infty} x^{s} f(x) \mathrm{d} x,
$$

with respect to the energy variable, for which the relations

$$
\mathcal{M}\left[a_{\mathrm{NC}} E^{\beta_{\mathrm{NC}}} \phi(E, t)\right](s, t)=a_{\mathrm{NC}} \mathcal{M}[\phi]\left(s+\beta_{\mathrm{NC}}, t\right)
$$

and

$$
\begin{aligned}
\mathcal{M}\left[\int_{0}^{1} \frac{\mathrm{d} y}{1-y} a_{\mathrm{NC}}\right. & \left.\left(\frac{E}{1-y}\right)^{\beta_{\mathrm{NC}}} f(y) \phi\left(\frac{E}{1-y}, t\right)\right](s, t) \\
& =a_{\mathrm{NC}} \int_{0}^{1} \mathrm{~d} y(1-y)^{s} f(y) \cdot \mathcal{M}[\phi]\left(s+\beta_{\mathrm{NC}}, t\right)
\end{aligned}
$$


hold, as can be shown by a straightforward calculation. Applying these and completely analogous relations to Eqs. 3.17 and 3.18 results in

$$
\begin{array}{rl}
\frac{\partial \mathcal{M}[\phi](s, t)}{\partial t}=-a_{\mathrm{NC}} & \mathcal{M}[\phi]\left(s+\beta_{\mathrm{NC}}, t\right)-a_{\mathrm{CC}} \mathcal{M}[\phi]\left(s+\beta_{\mathrm{CC}}, t\right) \\
& +a_{\mathrm{NC}} \int_{0}^{1}(1-y)^{s} f(y) \mathrm{d} y \cdot \mathcal{M}[\phi]\left(s+\beta_{\mathrm{NC}}, t\right) \\
& +b_{\mathrm{CC}} \int_{0}^{1}(1-y)^{s} s(y) \mathrm{d} y \cdot \mathcal{M}[\pi]\left(s+\alpha_{\mathrm{CC}}, t\right) \\
& +b_{\mathrm{CC}} \int_{0}^{1}(1-y)^{s} h(y) \mathrm{d} y \cdot P(t) \mathcal{M}[\pi](s+\kappa, t)
\end{array}
$$

and

$$
\begin{array}{rl}
\frac{\partial \mathcal{M}[\pi](s, t)}{\partial t}=-b_{\mathrm{dec}} & P(t) \mathcal{M}[\pi](s+\kappa, t)-\chi \mathcal{M}[\pi](s, t) \\
& -b_{\mathrm{NC}} \mathcal{M}[\pi]\left(s+\alpha_{\mathrm{NC}}, t\right)-b_{\mathrm{CC}} \mathcal{M}[\pi]\left(s+\alpha_{\mathrm{CC}}, t\right) \\
& +\alpha_{\mathrm{CC}} \int_{0}^{1}(1-y)^{s} g(y) \mathrm{d} y \cdot \mathcal{M}[\phi]\left(s+\beta_{\mathrm{CC}}, t\right) \\
& +\int_{0}^{1}(1-y)^{s} \xi_{0}(y) \mathrm{d} y \cdot \mathcal{M}[\pi](s, t) \\
& +b_{\mathrm{NC}} \int_{0}^{1}(1-y)^{s} r(y) \mathrm{d} y \cdot \mathcal{M}[\pi]\left(s+\alpha_{\mathrm{NC}}, t\right) .
\end{array}
$$

These equations admittedly still look somewhat awkward. However, we may further simplify them by applying the Laplace transform [150]

$$
\mathcal{L}[f](\lambda) \equiv \int_{0}^{\infty} e^{-\lambda x} f(x) \mathrm{d} x \quad(\lambda \in \mathbb{C}),
$$

which works reasonably well on the left hand sides, as a partial integration shows that the Laplace transform of a derivative is given by

$$
\mathcal{L}[\partial f / \partial t](\lambda)=\lambda \mathcal{L}[f](\lambda)-f(0) .
$$

But, alas, on the right hand sides we meet a serious drawback: There is no simple general form for the Laplace transform with an arbitrary $P(t)$. Hence we resort to assuming that $P(t)$ is a sum of exponential functions,

$$
P(t)=\sum_{k=1}^{n} P_{k} e^{-\delta_{k} t}
$$


While at first sight this seems somewhat restrictive, from a pragmatic point of view it is basically more than we would need. Indeed, an accurate description of the tauon decay (which gives rise to the $P(t)$ ) is necessary for short distances only. These will typically involve a mountain or the atmosphere, for which the density should be constant or change exponentially, so that both cases are encompassed by Eq. 3.21.

With this approximation, we obtain

$$
\mathcal{L}[P(t) f(t)](\lambda)=\sum_{k=1}^{n} P_{k} \mathcal{L}[f]\left(\lambda+\delta_{k}\right) .
$$

In order to avoid as many cumbersome terms as possible, we define the functions

$$
F(s) \equiv \int_{0}^{1}(1-y)^{s} f(y) \mathrm{d} y
$$

and, completely analogously, $S(s), G(s), H(s), \Xi(s)$ and $R(s)$. Together with the definition

$$
\mathcal{N}[f] \equiv \mathcal{L}[\mathcal{M}[f]]
$$

Eqs. 3.19 and 3.20 may then be transformed into

$$
\begin{array}{rl}
\lambda \mathcal{N}[\phi](s, \lambda)-\mathcal{M}[\phi](s, 0)=-a_{\mathrm{NC}} & \mathcal{N}[\phi]\left(s+\beta_{N C}, \lambda\right)-a_{\mathrm{CC}} \mathcal{N}[\phi]\left(s+\beta_{\mathrm{CC}}, \lambda\right) \\
& +a_{\mathrm{NC}} F(s) \mathcal{N}[\phi]\left(s+\beta_{\mathrm{NC}}, \lambda\right) \\
& +b_{\mathrm{CC}} S(s) \mathcal{N}[\pi]\left(s+\alpha_{\mathrm{CC}}, \lambda\right) \\
& +\sum_{k=1}^{n} P_{k} b_{\mathrm{dec}} H(s) \mathcal{N}[\pi]\left(s+\kappa, \lambda+\delta_{k}\right)
\end{array}
$$

and

$$
\begin{aligned}
\lambda \mathcal{N}[\pi](s, \lambda)-\mathcal{M}[\pi](s, 0)=- & \sum_{k=1}^{n} P_{k} b_{\mathrm{dec}} \mathcal{N}[\pi]\left(s+\kappa, \lambda+\delta_{k}\right)-\chi \mathcal{N}[\pi](s, \lambda) \\
& -b_{\mathrm{NC}} \mathcal{N}[\pi]\left(s+\alpha_{\mathrm{NC}}, \lambda\right)-b_{\mathrm{CC}} \mathcal{N}[\pi]\left(s+\alpha_{\mathrm{CC}}, \lambda\right) \\
& +a_{\mathrm{CC}} G(s) \mathcal{N}[\phi]\left(s+\beta_{C C}, \lambda\right)+\Xi(s) \mathcal{N}[\phi](s, \lambda) \\
& +b_{\mathrm{NC}} R(s) \mathcal{N}[\pi]\left(s+\alpha_{\mathrm{NC}}, \lambda\right)
\end{aligned}
$$


It should be noted that $\mathcal{M}[\phi](s, 0)$ and $\mathcal{M}[\pi](s, 0)$ are the Mellin transforms of the initial fluxes and thus constitute known functions. Hence we have managed to reduce the original set of integro-differential equations to a set of difference equations. In order to be able to give a formal solution, we employ three further assumptions: Firstly, we assume that only the neutrino-nucleon cross sections are relevant. Secondly, we take $\beta_{\mathrm{CC}}=\beta_{\mathrm{NC}} \equiv \beta$. And thirdly, we assume the density is constant. Then we get

$$
\begin{aligned}
& \lambda \mathcal{N}[\phi](s, \lambda)-\mathcal{M}[\phi](s, 0) \\
& =-\left(a_{\mathrm{NC}}+a_{\mathrm{CC}}\right) \mathcal{N}[\phi](s+\beta, \lambda)+a_{\mathrm{NC}} F(s) \mathcal{N}[\phi](s+\beta, \lambda) \\
& +P_{0} b_{\mathrm{dec}} H(s) \mathcal{N}[\pi](s+\kappa, \lambda) \\
& \lambda \mathcal{N}[\pi](s, \lambda)-\mathcal{M}[\pi](s, 0) \\
& =-P_{0} b_{\operatorname{dec}} \mathcal{N}[\pi](s+\kappa, \lambda)+a_{\mathrm{CC}} G(s) \mathcal{N}[\phi](s+\beta, \lambda)
\end{aligned}
$$

where obviously $P_{0}$ is the inverse of the target nucleon number density (cf. Eq. 3.13). While unfortunately the solution of Eqs. 3.24 and 3.25 remains quite awkward, we may at least outline a formal solution. To this end, we use $f$ and $g$ instead of $\mathcal{N}[\phi]$ and $\mathcal{N}[\pi]$, respectively, and define the matrix

$$
M(s, \lambda) \equiv\left(\begin{array}{cccccccccc}
\lambda & 0 & F_{11} & F_{12} & 0 & 0 & 0 & 0 & 0 & \ldots \\
0 & \lambda & F_{21} & F_{22} & 0 & 0 & 0 & 0 & 0 & \ldots \\
0 & 0 & \lambda & 0 & F_{11, \beta} & F_{12, \beta} & 0 & 0 & 0 & \ldots \\
0 & 0 & 0 & \lambda & 0 & 0 & F_{21, \beta} & F_{22, \beta} & 0 & \ldots \\
0 & 0 & 0 & 0 & \lambda & 0 & 0 & 0 & F_{11, \kappa} & \ldots \\
0 & 0 & 0 & 0 & 0 & \lambda & 0 & 0 & 0 & \ldots \\
\ldots & \ldots & \ldots & \ldots & \ldots & \ldots & \ldots & \ldots & \ldots & \ldots
\end{array}\right),
$$

where the main diagonal elements are equal to $\lambda$, each row contains two " $\mathrm{F}$ terms" (lying next to each other), and in the $n$-th row $(n \geqslant 3)$, the first $\mathrm{F}$ term constitutes the $(2 n-1)$-th element. The $\mathrm{F}$ terms (from left to right) are given by

$$
\begin{aligned}
& F_{11}, F_{12}, F_{21}, F_{22}, F_{11, \beta}, F_{12, \beta}, F_{21, \beta}, F_{22, \beta}, F_{11, \kappa}, F_{12, \kappa}, F_{21, \kappa}, F_{22, \kappa}, F_{11, \beta \beta}, \\
& F_{12, \beta \beta} F_{21, \beta \beta}, F_{22, \beta \beta}, F_{11, \beta \kappa}, \ldots, F_{22, \beta \kappa}, F_{11, \kappa \beta}, \ldots, F_{22, \kappa \beta}, F_{11, \kappa \kappa}, \ldots \\
& F_{11, \beta \beta \beta}, \ldots, F_{11, \beta \beta \kappa}, \ldots, F_{11, \beta \kappa \beta}, \ldots, F_{11, \beta \kappa \kappa}, \ldots, F_{11, \kappa \beta \beta}, \ldots
\end{aligned}
$$

and they are to be interpreted as $F_{i k} \equiv F_{i k}(s)$ and

$$
F_{i k, c_{1} c_{2} \ldots c_{n}} \equiv F_{i k}\left(s+c_{1}+c_{2}+\ldots+c_{n}\right)
$$


with

$$
\begin{aligned}
& F_{11}(s) \equiv a_{\mathrm{NC}}+a_{\mathrm{CC}}-a_{\mathrm{NC}} F(s) \\
& F_{12}(s) \equiv-P_{0} b_{\mathrm{dec}} H(s) \\
& F_{21}(s) \equiv-a_{\mathrm{CC}} G(s) \\
& F_{22}(s) \equiv P_{0} b_{\mathrm{dec}}
\end{aligned}
$$

In addition, we define the vector $\boldsymbol{v}$ as

$$
\begin{aligned}
& \boldsymbol{v}(s, \lambda)=(f(s), g(s), f(s+\beta), g(s+\beta), f(s+\beta+\beta), g(s+\beta+\kappa), \\
& \quad f(s+\kappa+\beta), g(s+\kappa+\kappa), f(s+\beta+\beta+\beta), g(s+\beta+\beta+\kappa), \ldots)^{\mathrm{T}}
\end{aligned}
$$

with $f(x) \equiv \mathcal{N}[\gamma](x, \lambda)$ and $g(x) \equiv \mathcal{N}[\pi](x, \lambda)$. Then Eqs. 3.24 and 3.25 can be rewritten in the compact form

$$
\boldsymbol{\omega}_{0}(s)=M(s, \lambda) \boldsymbol{v}(s, \lambda)
$$

where $\boldsymbol{\omega}_{0}(s)$ depends on the (known) functions $\mathcal{M}[\phi](s, 0)$ and $\mathcal{M}[\pi](s, 0)$. Obviously, this equation is equivalent to

$$
\boldsymbol{v}(s, \lambda)=M^{-1}(s, \lambda) \boldsymbol{\omega}_{0}(s)
$$

and as all we need to know is comprised within the first two elements of $\boldsymbol{v}$, we see that in order to solve the equation, we need to know the first two rows of $M^{-1}$. Their elements, however, can be obtained recursively.

Now an explicit calculation shows that the first eight elements of the first and second row of $M^{-1}$ are given by

$$
\left(\begin{array}{cccccccc}
\frac{1}{\lambda} & 0 & -\frac{1}{\lambda^{2}} F_{11} & -\frac{1}{\lambda^{2}} F_{12} & \frac{1}{\lambda^{3}} F_{11} F_{11, \beta} & \frac{1}{\lambda^{3}} F_{11} F_{12, \beta} & \frac{1}{\lambda^{3}} F_{12} F_{21, \kappa} & \frac{1}{\lambda^{3}} F_{12} F_{22, \kappa} \\
0 & \frac{1}{\lambda} & -\frac{1}{\lambda^{2}} F_{21} & -\frac{1}{\lambda^{2}} F_{22} & \frac{1}{\lambda^{3}} F_{21} F_{11, \beta} & \frac{1}{\lambda^{3}} F_{21} F_{12, \beta} & \frac{1}{\lambda^{3}} F_{22} F_{21, \kappa} & \frac{1}{\lambda^{3}} F_{22} F_{22, \kappa}
\end{array}\right)
$$

and that for either of the two rows, all the other elements $E_{k i}(k=1,2)$ can 
be obtained recursively by means of the relations

$$
\begin{array}{rlrl}
E_{k, 2^{n}+1} & =-\frac{1}{\lambda} F_{11,\langle 1\rangle} E_{k, 2^{n-1}+1} & E_{k, 2^{n}+2} & =-\frac{1}{\lambda} F_{12,\langle 1\rangle} E_{k, 2^{n-1}+1} \\
E_{k, 2^{n}+3} & =-\frac{1}{\lambda} F_{21,\langle 2\rangle} E_{k, 2^{n-1}+2} & E_{k, 2^{n}+4} & =-\frac{1}{\lambda} F_{22,\langle 2\rangle} E_{k, 2^{n-1}+2} \\
E_{k, 2^{n}+5} & =-\frac{1}{\lambda} F_{11,\langle 3\rangle} E_{k, 2^{n-1}+3} & E_{k, 2^{n}+6} & =-\frac{1}{\lambda} F_{12,\langle 3\rangle} E_{k, 2^{n-1}+3} \\
E_{k, 2^{n}+7} & =-\frac{1}{\lambda} F_{21,\langle 4\rangle} E_{k, 2^{n-1}+4} & E_{k, 2^{n}+8} & =-\frac{1}{\lambda} F_{22,\langle 4\rangle} E_{k, 2^{n-1}+4} \\
\ldots & \ldots & \\
E_{k, 2^{n+1}-1} & =-\frac{1}{\lambda} F_{21,\left\langle 2^{n-1}\right\rangle} E_{k, 2^{n}} & E_{k, 2^{n+1}} & =-\frac{1}{\lambda} F_{22,\left\langle 2^{n-1}\right\rangle} E_{k, 2^{n}}
\end{array}
$$

where $\langle 1\rangle \equiv \beta \beta \ldots \beta \beta,\langle 2\rangle \equiv \beta \beta \ldots \beta \kappa,\langle 3\rangle \equiv \beta \beta \ldots \kappa \beta,\langle 4\rangle \equiv \beta \beta \ldots \kappa \kappa$, $\ldots,\left\langle 2^{n-1}-1\right\rangle \equiv \kappa \kappa \ldots \kappa \beta$ and $\left\langle 2^{n-1}\right\rangle \equiv \kappa \kappa \ldots \kappa \kappa$. Eq. 3.26 then yields the first two elements of $\boldsymbol{v}$, and an inverse Laplace and Mellin transform gives the desired tau neutrino and tauon fluxes.

Hence we have managed to obtain a formal solution of the cascade equation. Alas, it turns out to be rather infeasible for any practical calculation. Things simplify a little bit, if we assume instantaneous tauon decay and approximate the integrals, as we will show in the next subsection.

\subsubsection{Approximate approach}

In the last section, we have investigated the integral transformation approach to the cascade equations for both tau neutrinos and tauons. However, for energies below $\sim 10^{8} \mathrm{GeV}$ it is an excellent approximation to assume that the tauons decay instantaneously. Then the need for a separate cascade equation for the tauons vanishes, and the cascade equation for the tau neutrino flux simplifies to

$$
\frac{\partial \phi(E, t)}{\partial t}=-\sigma_{\mathrm{tot}}(E) \phi(E, t)+\int_{E}^{\infty} \frac{\mathrm{d} E^{\prime}}{E^{\prime}} \frac{\mathrm{d} \sigma\left(E^{\prime}, y\right)}{\mathrm{d} y} \phi\left(E^{\prime}, t\right)
$$

where the "cross section" $\mathrm{d} \sigma / \mathrm{d} y$ includes both the neutral current interactions and the charged current interactions with subsequent tauon decay. We 
may Laplace transform Eq. 3.32 to give

$$
\lambda \mathcal{L}[\phi](E, \lambda)-\phi(E, 0)=-\sigma_{\text {tot }}(E) \mathcal{L}[\phi](E, \lambda)+\int_{E}^{\infty} \frac{\mathrm{d} E^{\prime}}{E^{\prime}} \frac{\mathrm{d} \sigma\left(E^{\prime}, y\right)}{\mathrm{d} y} \mathcal{L}[\phi]\left(E^{\prime}, \lambda\right)
$$

Without real loss of generality, the neutrino flux spectrum may be taken to vanish above a sufficiently high energy $\epsilon_{\max }$. If we use the definitions $x \equiv E^{\prime} / \epsilon_{\max }$ and

$$
\begin{aligned}
F(x, \lambda) & \equiv \mathcal{L}[\phi]\left(\epsilon_{\max } x, \lambda\right) & \Sigma_{\text {tot }}(x) & \equiv \sigma_{\text {tot }}\left(\epsilon_{\max } x\right) \\
\phi(x) & \equiv \phi\left(\epsilon_{\max } x, 0\right) & \Sigma\left(x^{\prime}, x\right) & \equiv \frac{1}{x^{\prime}} \frac{\mathrm{d} \sigma\left(\epsilon_{\max } x^{\prime}, 1-x / x^{\prime}\right)}{\mathrm{d} y}
\end{aligned}
$$

we may (keeping in mind that $y=1-x / x^{\prime}$ ) rewrite Eq. 3.33 as

$$
F(x, \lambda)=\frac{1}{\lambda+\Sigma_{\text {tot }}(x)}\left(\phi(x)+\int_{x}^{1} \mathrm{~d} x^{\prime} \Sigma\left(x^{\prime}, x\right) F\left(x^{\prime}, \lambda\right)\right) .
$$

In order to find an approximate solution of this equation, we compute $F$ for a fixed set

$$
\left\{x_{i} \mid i=1,2,3, \ldots, N ; x_{1}=1 ; x_{i+1}<x_{i} ; x_{N}=0\right\}
$$

of values for $x$ and assume that for the sake of obtaining the integral, $F(x, \lambda)$ may be replaced by a linear function between consecutive $x_{i}$ 's. In other words, we assume that

$$
F(x, \lambda)=F\left(x_{i}, \lambda\right)+\frac{F\left(x_{i+1}, \lambda\right)-F\left(x_{i}, \lambda\right)}{x_{i+1}-x_{i}}\left(x-x_{i}\right) \quad\left(x \in\left[x_{i+1}, x_{i}\right]\right),
$$

and an explicit calculation shows that for the $F\left(x_{i}, \lambda\right)$ the relations

$$
F(1, \lambda)=\frac{\phi(1)}{1+\Sigma_{\operatorname{tot}(1)}}
$$




$$
\begin{aligned}
F\left(x_{n}, \lambda\right) & =\frac{1}{\lambda+\Sigma_{\text {tot }}\left(x_{n}\right)}\left(\phi\left(x_{n}\right)+\sum_{i=1}^{n-1} \int_{x_{i+1}}^{x_{i}} \mathrm{~d} x^{\prime} \Sigma\left(x^{\prime}, x_{n}\right) F\left(x^{\prime}, \lambda\right) \mathrm{d} x^{\prime}\right) \\
= & \frac{1}{\lambda+\sum_{\text {tot }}\left(x_{n}\right)}\left(\phi\left(x_{n}\right)\right. \\
& +\sum_{i=1}^{n-1}\left\{\left[-\frac{x_{i}}{x_{i+1}-x_{i}} W_{0}^{x_{i+1}, x_{i}}\left(x_{n}\right)+\frac{1}{x_{i+1}-x_{i}} W_{1}^{x_{i+1}, x_{i}}\left(x_{n}\right)\right] F\left(x_{i+1}, \lambda\right)\right. \\
+ & {\left.\left.\left[-\frac{1}{x_{i+1}-x_{i}} W_{1}^{x_{i+1}, x_{i}}\left(x_{n}\right)+\frac{x_{i+1}}{x_{i+1}-x_{i}} W_{0}^{x_{i+1}, x_{i}}\left(x_{n}\right)\right] F\left(x_{i}, \lambda\right)\right\}\right) }
\end{aligned}
$$

are fulfilled, where

$$
W_{0}^{a, b}(x) \equiv \int_{a}^{b} \Sigma\left(x^{\prime}, x\right) \mathrm{d} x^{\prime}
$$

and

$$
W_{1}^{a, b}(x) \equiv \int_{a}^{b} x^{\prime} \Sigma\left(x^{\prime}, x\right) \mathrm{d} x^{\prime}
$$

Eq. 3.35 can be solved for $F\left(x_{n}, \lambda\right)$, and thus together with Eq. 3.34 it offers the possiblity to calculate $F\left(x_{n}, \lambda\right)$ recursively. The result is of the form

$$
F\left(x_{n}, \lambda\right)=\frac{a_{0}\left(x_{n}\right)+a_{1}\left(x_{n}\right) \lambda+a_{2}\left(x_{n}\right) \lambda^{2}+\ldots+a_{N}\left(x_{n}\right) \lambda^{N}}{\left(\lambda+b_{1}\right)\left(\lambda+b_{2}\right) \cdot \ldots \cdot\left(\lambda+b_{N-1}\right)\left(\lambda+b_{N}\right)}
$$

with

$$
b_{i}=\left\{\begin{array}{ll}
\Sigma_{\mathrm{tot}}(1) & (i=1) \\
\Sigma_{\mathrm{tot}}\left(x_{i}\right)+\left(\frac{x_{i-1}}{x_{i}-x_{i-1}} W_{0}^{x_{i}, x_{i-1}}\left(x_{i}\right)-\frac{1}{x_{i}-x_{i-1}} W_{i}^{x_{i}, x_{i-1}}\left(x_{i}\right)\right) & (i>1)
\end{array} .\right.
$$

We may abbreviate the nominator of Eq. 3.36 by means of the function $P_{N}$ defined as

$$
P_{N}\left(x_{n}\right) \equiv a_{0}\left(x_{n}, \lambda\right)+a_{1}\left(x_{n}\right) \lambda+a_{2}\left(x_{n}\right) \lambda^{2}+\ldots+a_{N}\left(x_{n}\right) \lambda^{N} .
$$


As uniform convergence is guaranteed for $P_{N}$ as a function of $\lambda$, one may apply the Jordan lemma [130] to perform the complex integration for the inverse Laplace transform. One then obtains the result

$$
\phi\left(x_{n}, t\right)=\sum_{k=1}^{N} \frac{P_{N}\left(-b_{k}\right)}{\prod_{\substack{i=1 \\ i \neq k}}^{N}\left(b_{i}-b_{k}\right)} e^{-b_{k} t}
$$

Hence we have managed to obtain the solution for the tau neutrino flux. Looking back, we see that this was achieved by using an approximation for its Laplace transform. However, we might as well try and approximate the cascade equation rather than the solution. This task will be undertaken in the next section.

\subsection{Discretizing the cascade equation}

So far, we have discussed the (tau) neutrino propagation by means of solving the respective cascade equation(s). Alas, it has become evident that this method requires various approximations, and even so, obtaining the neutrino flux proved to be be somewhat tedious.

However, we may get round the need of discussing integro-differential equations by using a discretized energy. This can formally be implemented in the cascade equations by assuming that the various cross sections involved can be expressed as a sum of delta functions, i.e. that they may be written in the form

$$
\frac{\mathrm{d} \sigma\left(E, E^{\prime}\right)}{\mathrm{d} E}=\sum_{i=1}^{M} B_{i}(E) \delta\left(E^{\prime}-E_{i}(E)\right),
$$

where the $B_{i}$ and $E_{i}(E)$ are chosen appropriately. As before, we'll perform the calculation for an instantaneous tauon decay. Hence, if we rewrite Eq. 3.32 in terms of the (final) energy rather than inelasticity parameter,

$$
\frac{\partial \phi(E, t)}{\partial t}=-\sigma_{\mathrm{tot}}(E) \phi(E, t)+\int_{E}^{\infty} \mathrm{d} E^{\prime} \frac{\mathrm{d} \sigma\left(E^{\prime}, E\right)}{\mathrm{d} E} \phi\left(E^{\prime}, t\right)
$$

we see by inserting Eq. 3.38 that the cascade equation simplifies to

$$
\frac{\partial \phi(E, t)}{\partial t}=-\sigma_{\mathrm{tot}}(E) \phi(E, t)+\sum_{i=1}^{\tilde{M}} \tilde{B}_{i}(E) \phi\left(\tilde{E}_{i}(E), t\right) .
$$


with functions $\tilde{B}_{i}, \tilde{E}_{i}$ and an integer $\tilde{M}$. Now let us consider a set $R$ of $N+1$ energies (labeled from 0 to $N$ ), which are ordered by decreasing value, and let us assume that for any $E_{n} \in R$, the energies $E\left(E_{n}\right)$ with a non-vanishing cross section are given by the subset $\left\{E_{i} \mid i<n\right\}$ of $R$. Then with the notation $A_{n} \equiv \sigma_{\text {tot }}\left(E_{n}\right)$ and $B_{\text {in }} \equiv B_{i}\left(E_{n}\right)$, Eq. 3.40 can be put in the form

$$
\frac{\partial \phi\left(E_{n}, t\right)}{\partial t}=-A_{n} \phi\left(E_{n}, t\right)+\sum_{i=0}^{n-1} B_{i n} \phi\left(E_{i}, t\right) .
$$

Accordingly the task of solving an integro-diferential equation has been boiled down to that of finding the solution of a set of $N$ coupled ordinary differential equations. The latter, however, can be performed analytically. Indeed, the neutrino flux $\phi$ described by Eq. 3.41 is given by

$$
\phi\left(E_{n}, t\right)=\sum_{i=0}^{n} \phi_{n}^{(i)} e^{-A_{i} t}
$$

where the coefficients $\phi_{n}^{(i)}$ are defined as

$$
\phi_{n}^{(i)} \equiv\left\{\begin{array}{ll}
\sum_{r=0}^{n-1} \frac{B_{r n}}{A_{n}-A_{i}} \phi_{r}^{(i)} & (i<n) \\
\phi\left(E_{n}, 0\right)-\sum_{k=0}^{n-1} \sum_{r=0}^{n-1} \frac{B_{r n}}{A_{n}-A_{k}} \phi_{r}^{(k)} & (i=n) \\
0 & (i>n)
\end{array} .\right.
$$

The form of the expression for $\phi$ given in Eq. 3.42 shouldn't be too much of a surprise. After all, for some given energy, we have to consider the fluxes for the energies greater or equal to that energy, and these should change more or less like an exponential function.

In order to prove Eqs. 3.42 and 3.43, we use complete induction. To start with, consider the first (and thus largest) energy $E_{0}$. Here, the differential equation is simply

$$
\frac{\partial \phi\left(E_{0}, t\right)}{\partial t}=-A_{0} \phi\left(E_{0}, t\right)
$$

and hence has the solution

$$
\phi\left(E_{0}, t\right)=\phi\left(E_{0}, 0\right) e^{-A_{0} t}
$$


in agreement with our proposition, so that the basis is indeed true. Turning now to an arbitrary $n>0$, we see that we have to solve the more general equation Eq. 3.41, which can be done by means of the variation of parameters method: Inserting the ansatz $\phi\left(E_{n}, t\right) \equiv K(t) e^{-A_{n} t}$ into Eq. 3.41, we see that $K$ must fulfill the condition

$$
\frac{\mathrm{d} K(t)}{\mathrm{d} t}=\sum_{i=0}^{n-1} B_{i n} \phi_{i}(t) e^{A_{n} t} .
$$

But the induction hypothesis asserts that the $\phi_{i}$ on the right hand side are given by Eq. 3.42, so that we can write down the formula for $\mathrm{d} K / \mathrm{d} t$ more explicitly,

$$
\frac{\mathrm{d} K(t)}{\mathrm{d} t}=\sum_{i=0}^{n-1} e^{A_{n} t} B_{i n} \sum_{r=0}^{i} \phi_{i}^{(r)} e^{-A_{r} t}=\sum_{i=0}^{n-1} \sum_{r=0}^{n-1} B_{i n} \phi_{i}^{(r)} e^{\left(A_{n}-A_{r}\right) t} .
$$

In the last step, we have made use of the fact that $\phi_{i}^{(r)}=0$ for all $r>i$. An integration of Eq. 3.44 immediately yields

$$
K(t)=K_{0}+\sum_{i=0}^{n-1} \sum_{r=0}^{n-1} \frac{B_{i n}}{A_{n}-A_{r}} \phi_{i}^{(r)} e^{\left(A_{n}-A_{r}\right) t}
$$

with a constant $K_{0}$, so that we obtain

$$
\phi_{n}(t)=K(t) e^{-A_{n} t}=K_{0} e^{-A_{n} t}+\sum_{i=0}^{n-1} \sum_{r=0}^{n-1} \frac{B_{i n}}{A_{n}-A_{r}} \phi_{i}^{(r)} e^{-A_{r} t},
$$

and for $t=0$ we have

$$
\phi_{n}(0)=K_{0}+\sum_{i=0}^{n-1} \sum_{r=0}^{n-1} \frac{B_{i n}}{A_{n}-A_{r}} \phi_{i}^{(r)},
$$

from which we get the value of $K_{0}$. Employing Eq. 3.43, we arrive at

$$
\begin{aligned}
\phi_{n}(t) & =\left(\phi_{n}(0)-\sum_{i=0}^{n-1} \sum_{r=0}^{n-1} \frac{B_{i n}}{A_{n}-A_{r}} \phi_{i}^{(r)}\right) e^{-A_{n} t}+\sum_{i=0}^{n-1} \sum_{r=0}^{n-1} \frac{B_{i n}}{A_{n}-A_{r}} \phi_{i}^{(r)} e^{-A_{r} t} \\
& =\phi_{n}^{(n)} e^{-A_{n} t}+\sum_{r=0}^{n-1}\left(\sum_{i=0}^{n-1} \frac{B_{i n}}{A_{n}-A_{r}} \phi_{i}^{(r)}\right) e^{-A_{r} t} \\
& =\phi_{n}^{(n)} e^{-A_{n} t}+\sum_{r=0}^{n-1} \phi_{n}^{(r)} e^{-A_{r} t}=\sum_{r=0}^{n} \phi_{n}^{(r)} e^{-A_{r} t}
\end{aligned}
$$


which is just Eq. 3.42. Thus we have successfully completed the induction step and thus proved our assertion.

So far, we have confined ourselves to a single flavor. There is no need for this, though. Looking at the found solution, we note that the energy appears in arguments only, so that its value doesn't matter as long as we get the $A_{i}$ and $B_{i k}$ right. This means, however, that we may introduce formal energy values $E_{i}^{\text {formal }}$ via

$$
\begin{array}{ll}
E_{n}^{\text {formal }}\left(\nu_{e}\right)=E_{n} & E_{n}^{\text {formal }}\left(\bar{\nu}_{e}\right)=E_{n}+\frac{1}{2} \Delta E \\
E_{n}^{\text {formal }}\left(\nu_{\mu}\right)=E_{n}+\frac{1}{6} \Delta E & E_{n}^{\text {formal }}\left(\bar{\nu}_{\mu}\right)=E_{n}+\frac{2}{3} \Delta E \\
E_{n}^{\text {formal }}\left(\nu_{\tau}\right)=E_{n}+\frac{1}{3} \Delta E & E_{n}^{\text {formal }}\left(\bar{\nu}_{\tau}\right)=E_{n}+\frac{5}{6} \Delta E
\end{array}
$$

where $\Delta E$ is the smallest energy difference between neighboring energy values, $\Delta E=\min \left(\left\{E_{i}-E_{i+1} \mid i=0, \ldots, N-1\right\}\right)$ (cf. Fig. 3.2). If we describe the neutrino propagation in terms of these variables, the set of coupled equations describing the propagation with transitions between the various flavors can be put together into a (single) set of equations of the form of Eq. 3.41, which can be solved as in the case of one flavor discussed above. As soon as we have introduced cross sections of the form of Eq. 3.38, the method outlined in this section obviously can be considered to be analytical and thus leads to no further inaccuracy in the computed neutrino fluxes. In other words, the error in the results is solely due to that of the cross section approximation. In order to estimate the latter, one may assume that the energy in a cross section argument is known up to the difference between adjacent energy values only.

Hence, if the number of discretized energies is increased, the solution should get more accurate. While this is true in principle, however, there is an important caveat: As the energy differences get smaller, the corresponding differences of the $A_{n}$ will get smaller as well. This means that the $\phi_{i}^{(r)}$ get very large, and hence we finally end up computing small differences of large values. For sufficiently small energy differences, the numerical precision of the variables in a computer program implementing the method thus won't suffice any longer, and one obtains random results, as illustrated by Fig. 3.3. 


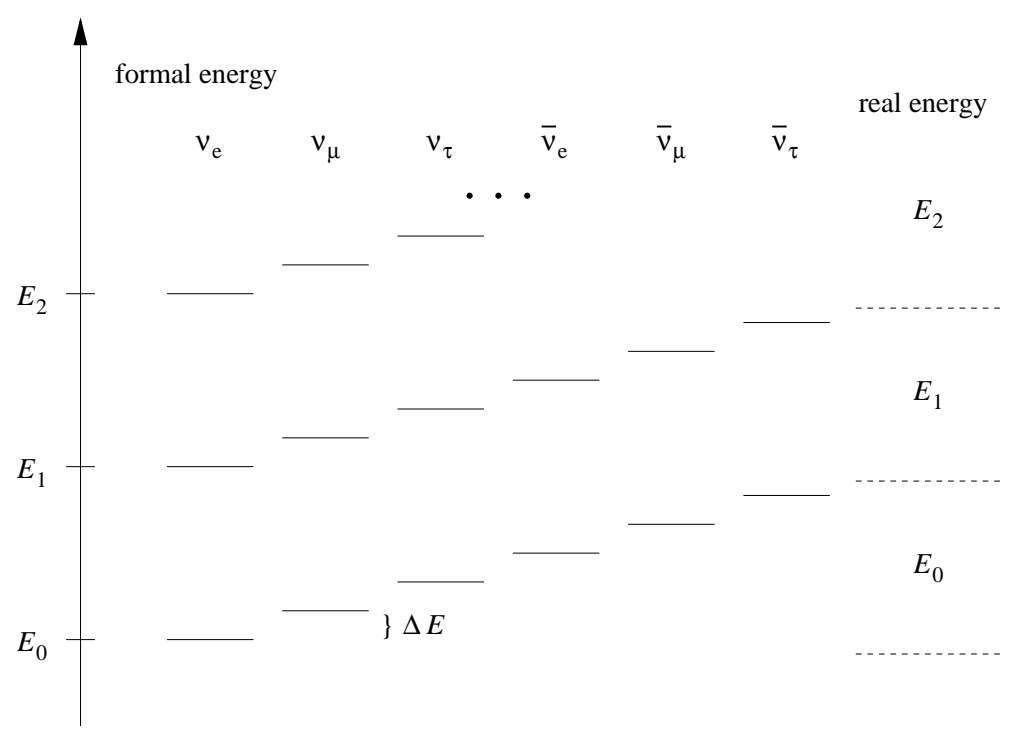

Figure 3.2: Schematic representation of the formal discretized energy values introduced in the main text. For simplicity, equidistant energies are assumed.
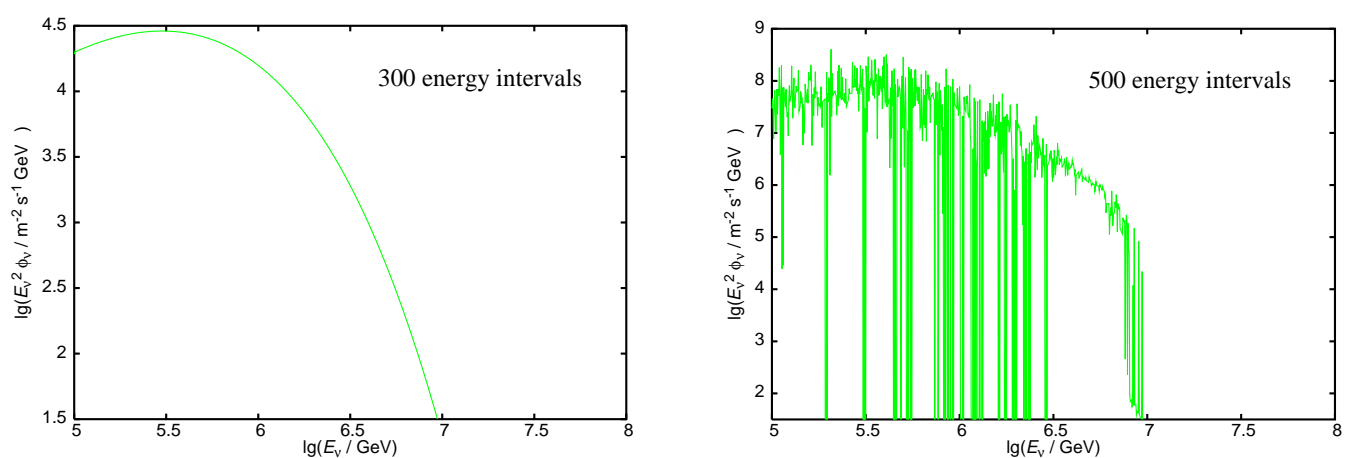

Figure 3.3: Left: Calculation of a neutrino flux with sufficiently large energy differences such that the numerical precision is sufficient. Right: If the energy differences are too small, the numerical precision is not sufficient for computing the small differences of the larges values encountered during the flux calculation. In this case the result becomes purely random. 


\subsection{Iteration}

The form of the cascade equations (cf. Eq. 3.39)

$$
\frac{\partial \phi(E, t)}{\partial t}=-\sigma_{\text {tot }}(E, t) \phi(E, t)+\int_{0}^{1} \frac{\mathrm{d} y}{1-y} \frac{\mathrm{d} \sigma(E /(1-y), y)}{\mathrm{d} y} \phi\left(\frac{E}{1-y}, t\right)
$$

describing neutrino propagation lends itself to an iterative ansatz: Inserting some initial guess for the solution (such as the solution for the case of absolutely no regeneration) in the integral on the right hand side yields the derivative of the neutrino flux, which can be integrated to give a second (hopefully improved) guess for the solution. This may then be reinserted on the right hand side, and the procedure is repeated until the computed flux has sufficiently converged.

In [153], a method for implementing this approach is outlined. Basically, one starts with introducing the $Z$ factor by demanding that the initial flux $\phi_{0}(E) \equiv \phi(E, 0)$ and the flux at some arbitrary $t$ are related by

$$
\phi(E, t)=\phi_{0}(E) \exp \left(-\frac{1-Z(E, t)}{\lambda(E)} t\right),
$$

where $\lambda(E) \equiv 1 / \sigma_{\text {tot }}(E)$. If furthermore $\Phi, \eta$, and $D$ are defined as

$$
\begin{gathered}
\Phi(E, y) \equiv \frac{1}{\sigma_{\text {tot }}(E)} \frac{\mathrm{d} \sigma(E /(1-y), y)}{\mathrm{d} y}, \\
\eta(E, y) \equiv \frac{\phi_{0}(E /(1-y))}{\phi_{0}(E)(1-y)}
\end{gathered}
$$

and

$$
D\left(E, E^{\prime}, t\right)=\frac{1-Z\left(E^{\prime}, t\right)}{\lambda\left(E^{\prime}\right)}-\frac{1-Z(E, t)}{\lambda(E)},
$$

Eq. 3.45 is equivalent to the equation

$$
Z(E, t)=\frac{1}{t} \int_{0}^{t} \mathrm{~d} t^{\prime} \int_{0}^{1} \mathrm{~d} y \eta(E, y) \Phi(E, y) e^{-t^{\prime} D\left(E, E /(1-y), t^{\prime}\right)}
$$


which may be solved by iteration, as described above. We will prove this assertion when discussing the accuracy of the iteration method. A reasonable choice for the initial guess is $Z^{(0)}(E) \equiv 0$, which corresponds to the case of no regeneration. The $(n+1)$-th iteration is obtained from the $n$-th iteration by means of the formula

$$
Z^{(n+1)}(E, x)=\frac{1}{t} \int_{0}^{t} \mathrm{~d} t^{\prime} \int_{0}^{1} \mathrm{~d} y \eta(E, y) \Phi(E, y) e^{-t^{\prime} D^{(n)}\left(E, E /(1-y), t^{\prime}\right)}
$$

with

$$
D^{(n)}\left(E, E^{\prime}, t\right)=\frac{1-Z^{(n)}\left(E^{\prime}, t\right)}{\lambda\left(E^{\prime}\right)}-\frac{1-Z^{(n)}(E, t)}{\lambda(E)} .
$$

In [153], a generalization to the case of more than one flavor is given, which explicitly includes a source function. There is, however, a more straightforward way, resembling our respective approach when discussing discretization.

The trick is to note that from a practical point of view, we may assume the existence of a maximum energy $E_{\max }$ above which all the neutrino fluxes vanish. Then we may introduce a formal energy for the various flavors (cf. Fig. 3.4),

$$
\begin{array}{ll}
E^{\text {formal }}\left(\nu_{e}\right)=E & E^{\text {formal }}\left(\bar{\nu}_{e}\right)=3 E_{\max }+E \\
E^{\text {formal }}\left(\nu_{\mu}\right)=E_{\max }+E & E^{\text {formal }}\left(\bar{\nu}_{\mu}\right)=4 E_{\max }+E \\
E^{\text {formal }}\left(\nu_{\tau}\right)=2 E_{\max }+E & E^{\text {formal }}\left(\bar{\nu}_{\tau}\right)=5 E_{\max }+E
\end{array}
$$

where $E$ denotes the "real" energy corresponding to the formal energies. As for the method of discretization discussed in the previous section, for these formal energies the (coupled) integro-differential flavors can be subsumed in a single equation of the form of Eq. 3.45, so that we can directly apply the formalism outlined in the preceding paragraphs. Corresponding results for the $Z$ factor of muon and tau neutrinos as a function of energy and depth are given in Figs. 3.5-3.8. An initial spectrum of power law form with spectral index -2 is assumed.

These plots illustrate three generic features of $Z$ worth noting. Firstly, the $Z$ factor generally has the tendency to decrease with energy and column number density. This can be explained by noting that the neutrino cross section and thus the neutrino losses increase with energy, so that the regeneration becomes less important at higher energies (as the reservoir of neutrinos that could be regenerated decreases more quickly). 


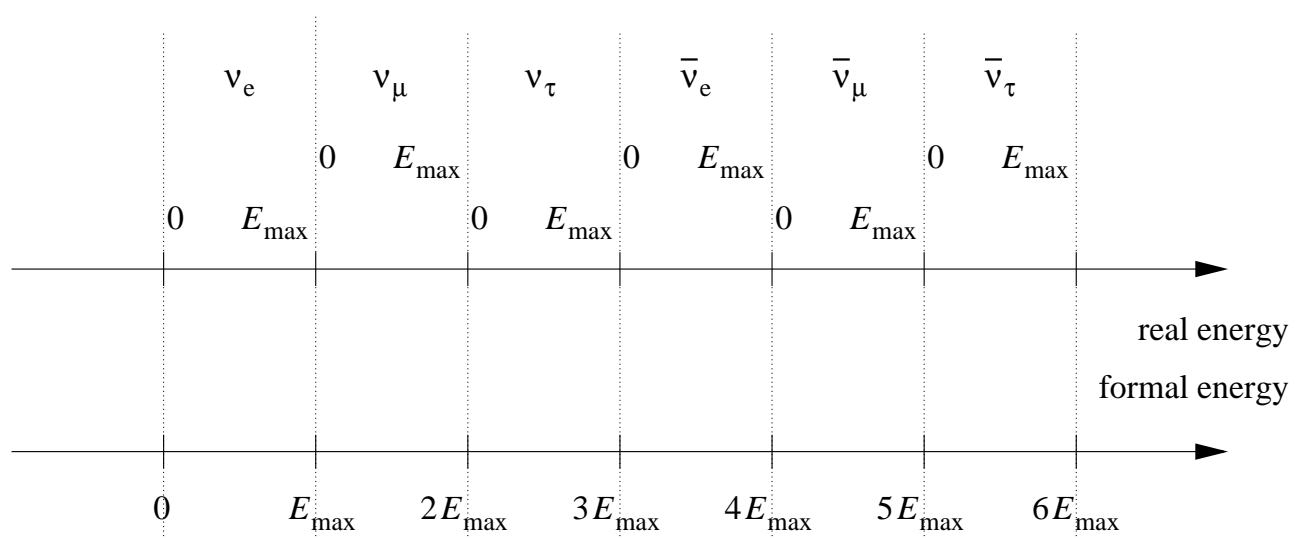

Figure 3.4: Schematic representation of the formal energies introduced in the main text.

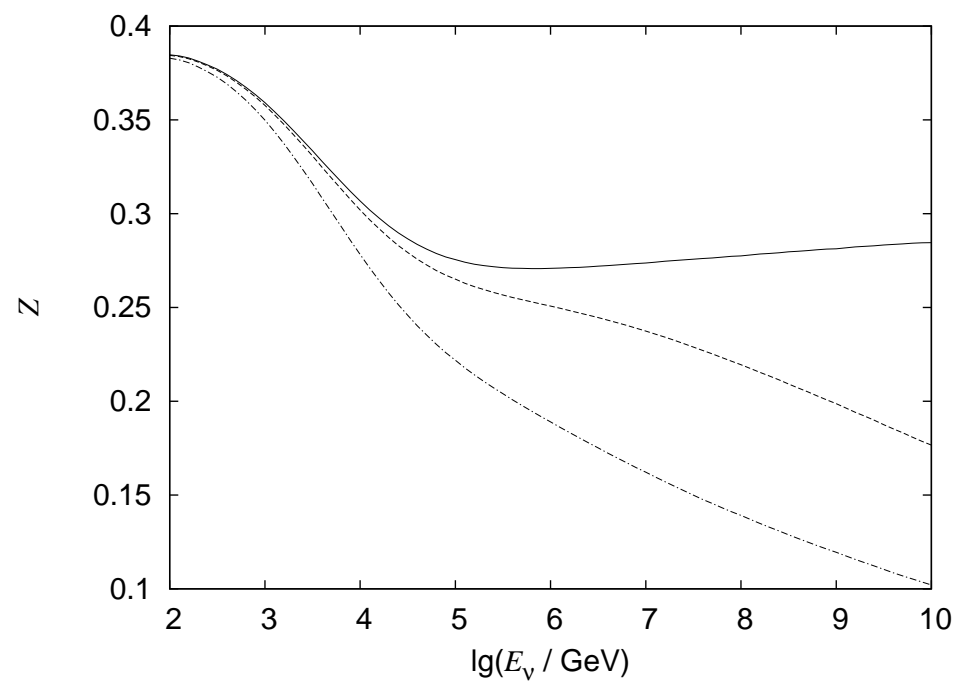

Figure 3.5: $Z$ factor of muon neutrinos as a function of the neutrino energy for an initial power law spectrum with index -2 . The column number densities $0 \mathrm{~cm}^{-2}$ (solid), $10^{33} \mathrm{~cm}^{-2}$ (dashed) and (corresponding to the full Earth diameter) $6.547 \times$ $10^{33} \mathrm{~cm}^{-2}$ (dot-dashed line) are assumed. Five iterations have been performed. 


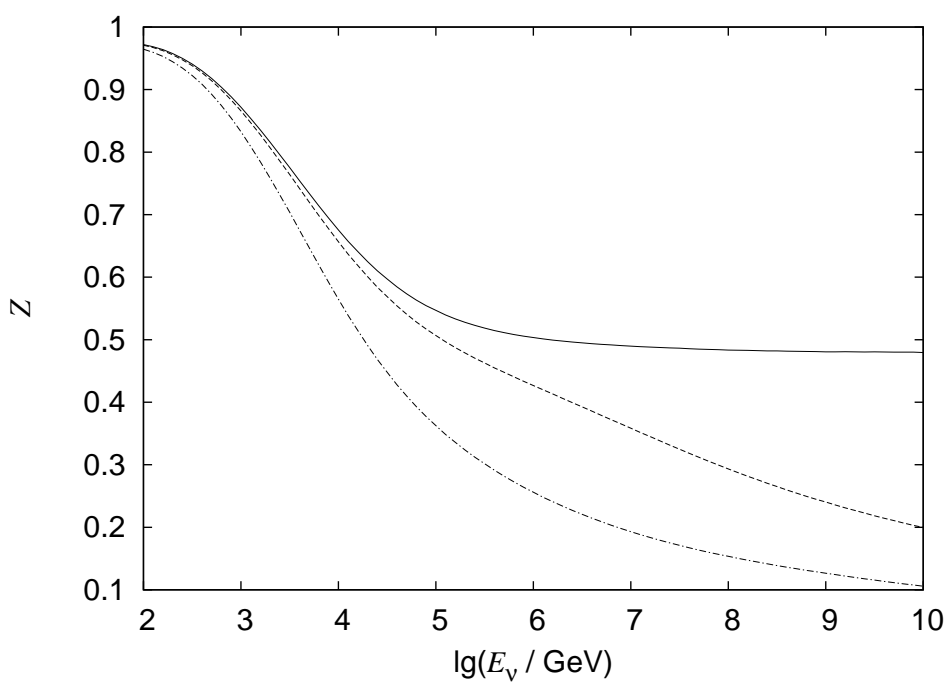

Figure 3.6: The same as Fig. 3.5, but for tau instead of muon neutrinos.

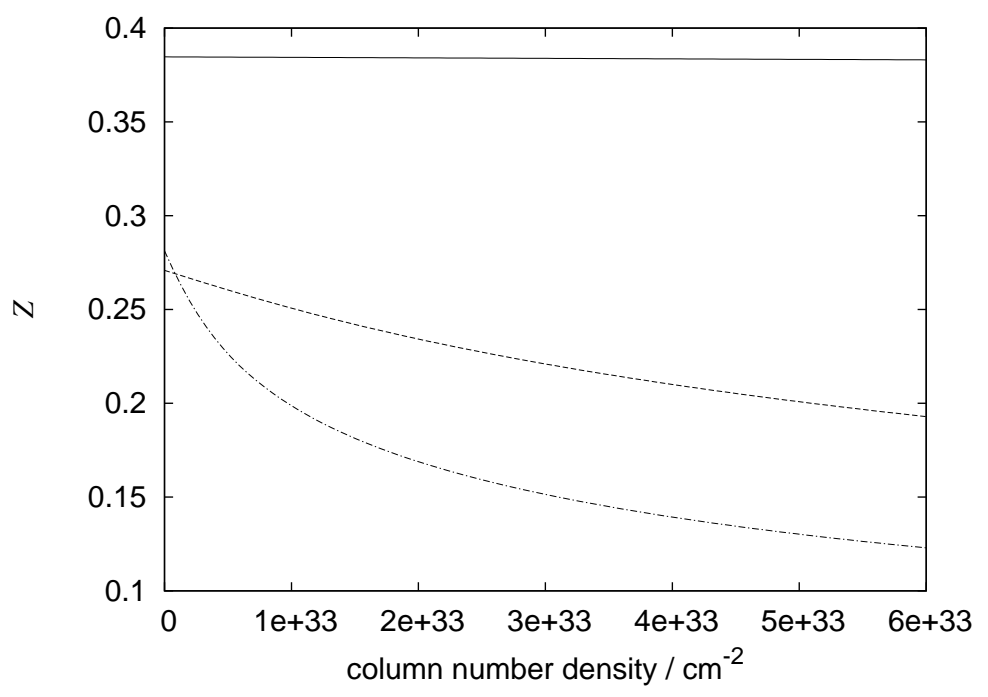

Figure 3.7: $Z$ factor of muon neutrinos as a function of the column number density for the neutrino energies $100 \mathrm{GeV}$ (solid), $10^{6} \mathrm{GeV}$ (dashed) and $10^{9} \mathrm{GeV}$ (dot-dashed line). Five iterations have been performed. 


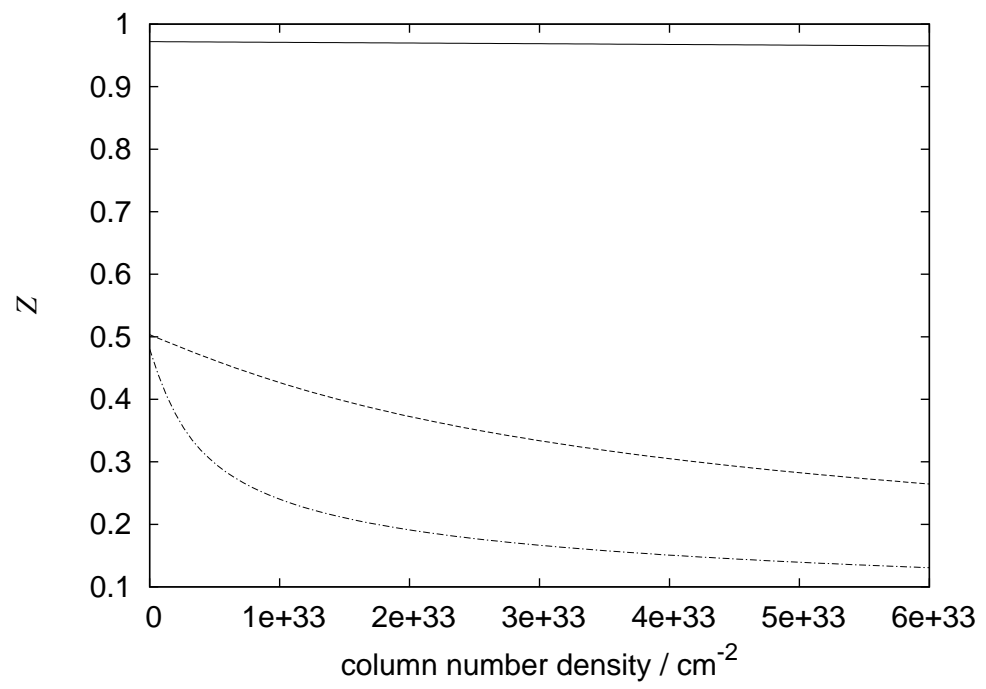

Figure 3.8: The same as Fig. 3.7, but for tau instead of muon neutrinos.

Secondly, the tau neutrino $Z$ factor is usually larger than the muon (or electron) neutrino one. This is no surprise, as for tau neutrinos the regeneration is more prominent, as charged current tau neutrino-nucleon interactions lead to no neutrino loss.

And thirdly, the $Z$ factor for small column number densities $t$ does not vanish. This might seem slightly astonishing; after all, for $t=0$ no neutrino interactions take place. However, this is ensured by the vanishing expontial factor $e^{-t \sigma_{\text {tot }}}$, independent of the value of $Z$.

Evidently, iterating $Z$ makes sense only, if the sequence of iterated functions actually converges. A hand-waving method for checking this is to compute the relative deviation

$$
r^{(k)}(E, t) \equiv\left|\frac{Z^{(k)}(E, t)-Z^{(\text {final iteration })}(E, t)}{Z^{(\text {final iteration })}(E, t)}\right|
$$

between the $k$-th and the largest considered iteration and to convince oneself that it approaches 0 for sufficiently large $k$. The quantity $r^{(k)}$ is shown for the $Z$ factors of Figs. $3.5-3.8$ in Figs. $3.9-3.12$, which clearly suggest that the fifth iteration constitutes a resonable approximation to the real $Z$.

The argument isn't entirely convincing, though, as we don't know how fast the distance between consecutive iterated functions will further decrease above the fifth iteration. And, worse yet, even if $\lim _{k \rightarrow \infty} r^{(k)}(E, t)=0$ holds, 


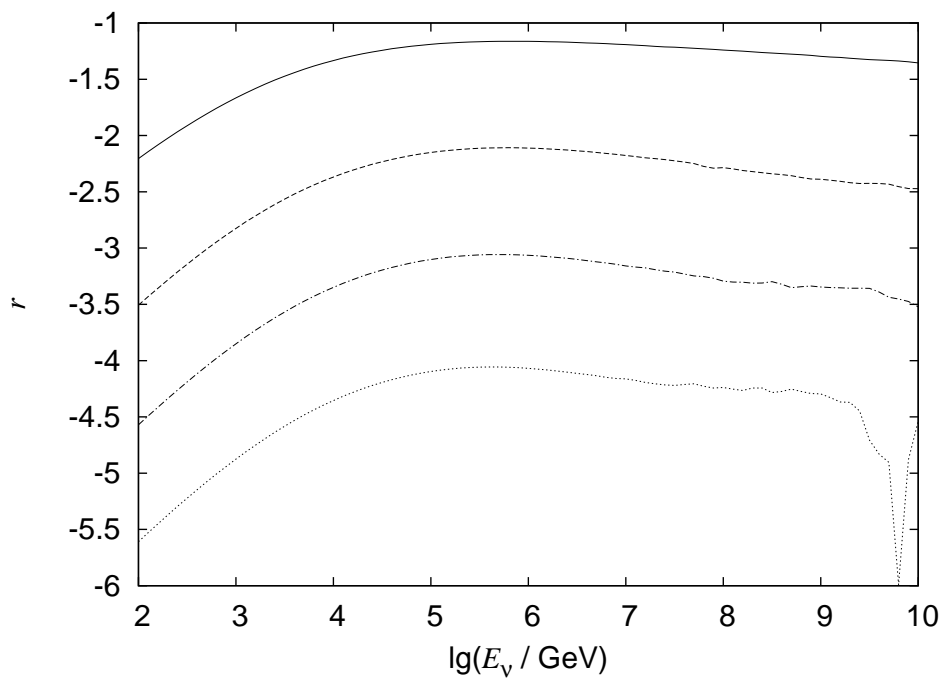

Figure 3.9: Relative accuracy $r$ of the muon neutrino $Z$ factor as a function of energy for the first (solid), second (dashed), third (dot-dashed), and fourth iteration (dotted line), as compared to the fifth iteration. $r$ has been computed for a column number density of $6.547 \times 10^{33} \mathrm{~cm}^{-2}$ (corresponding to the full Earth diameter).

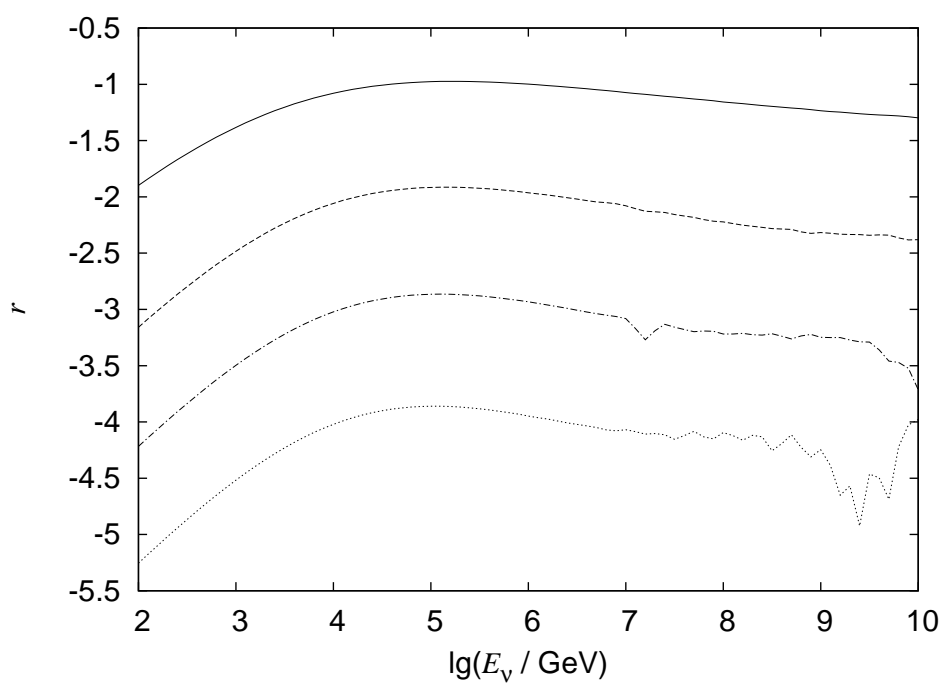

Figure 3.10: The same as Fig. 3.9, but for tau instead of muon neutrinos. 


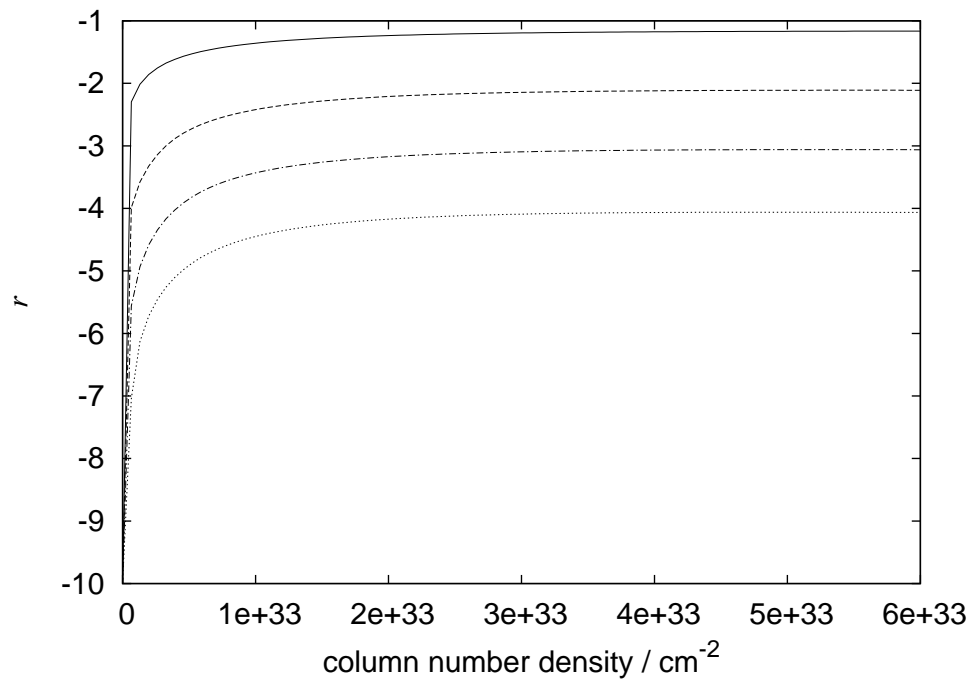

Figure 3.11: Relative accuracy $r$ of the muon neutrino $Z$ factor as a function of the column number density for the first (solid), second (dashed), third (dotdashed), and fourth iteration (dotted line), as compared to the fifth iteration. $r$ has been computed for an energy of $10^{6} \mathrm{GeV}$.

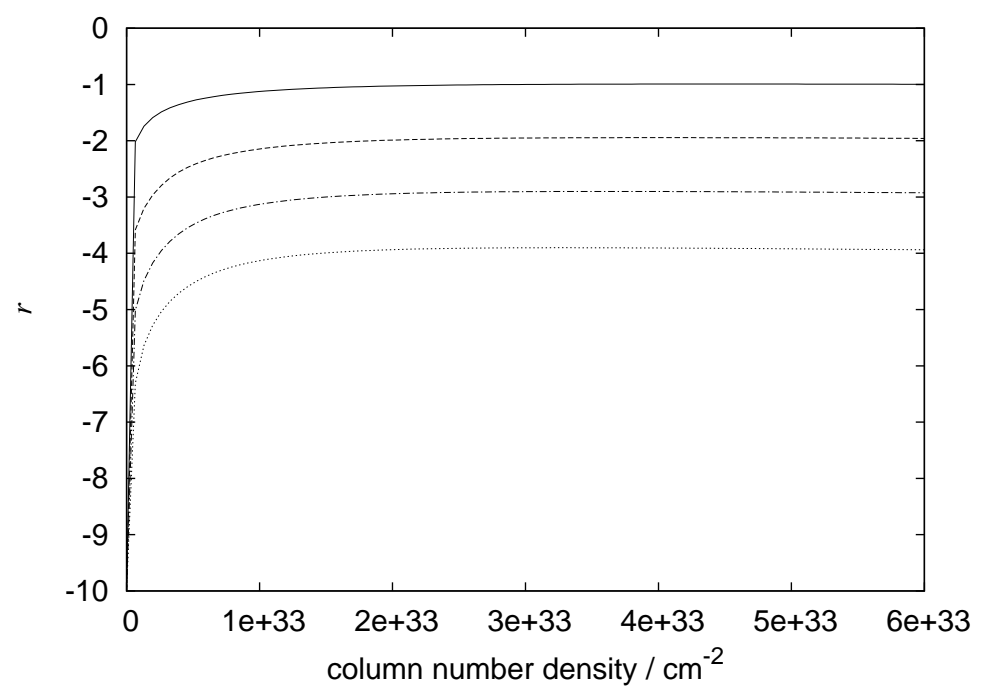

Figure 3.12: The same as Fig. 3.11, but for tau instead of muon neutrinos. 
we don't know whether the limit of the iterated $Z$ really is the solution of the cascade equation.

Hence we adopt a more direct approach to discussing the accuracy. To this end, we start with differentiating both sides of Eq. 3.46 with respect to $t$ :

$$
\frac{\partial \phi(E, t)}{\partial t}=\left(\frac{t}{\lambda(E)} \frac{\partial Z(E, t)}{\partial t}+\frac{Z(E, t)}{\lambda(E)}-\frac{1}{\lambda(E)}\right) \phi(E, t) .
$$

Inserting this relation into Eq. 3.45 and using the definition

$$
I(E, t) \equiv \int_{0}^{1} \Phi(E, y) \phi\left(\frac{E}{1-y}, y\right) \frac{\mathrm{d} y}{1-y}
$$

immediately yields

$$
\begin{aligned}
\frac{\partial \phi(E, t)}{\partial t} & =\left(\frac{t}{\lambda(E)} \frac{\partial Z(E, t)}{\partial t}+\frac{Z(E, t)}{\lambda(E)}-\frac{1}{\lambda(E)}\right) \phi(E, t) \\
& =\frac{I(E, t)}{\lambda(E)}-\frac{\phi(E, t)}{\lambda(E)}
\end{aligned}
$$

and thus

$$
\frac{\partial Z(E, t)}{\partial t}=\frac{I(E, t)}{t \phi(E, t)}-\frac{Z(E, t)}{t}
$$

It is straightforward to show that differentiating both sides of Eq. 3.47 leads to this equation as well, hence proving that assertion. Now let us assume that some calculated $Z(E, t)$ were exact for some $t$. Then the right hand side of Eq. 3.50 would be the true derivative of $Z$, whereas the left hand side would be the calculated derivative, so that their difference would be the error in the calculated derivative. This implies, however, that under the assumption that $Z(E, 0)$ is exact, the quantity $\Delta Z(E, t)$

$$
\Delta Z(E, t)=\int_{0}^{t}\left|\frac{I(E, t)}{t F(E, t)}-\frac{Z(E, t)}{t}-\frac{\partial Z(E, t)}{\partial t}\right| \mathrm{d} t
$$

constitutes a good estimate of the inaccuracy of the calculated $Z$ factor, at least if $\Delta Z \ll Z$. Hence some calculated $Z$ can be considered to be accurate if the relative accuracy $\rho(E, t)$ defined as

$$
\rho(E, t) \equiv \frac{\Delta Z(E, t)}{Z(E, t)}
$$


is much less than 1 for all relevant energies and column number densities. When computing this quantity, we use the method gsl_integration_qags of the GNU Scientific Library [95] and Ridder's method as implemented in [165] for obtaining $I$ and $\partial Z / \partial t$, respectively. The integration over the column number density $t$ may be performed by means of the trapezoidal rule, as we are interested in the order of magnitude only.

A subtle but important issue remains, namely whether the assumption that the calculated $Z(E, 0)$ is exact actually makes sense. If this were not the case, $\rho$ wouldn't be a measure for the accuracy of $Z$.

Looking at the definition of $Z$, Eq. 3.46, we note that $Z(E, 0)$ itself isn't defined, so that strictly speaking, in the preceding paragraphs $Z(E, 0)$ really means $\lim _{t \rightarrow 0} Z(E, t)$. Hence let us consider a small (but positive) column number density $t$. Then we see from Eq. 3.45 that $\phi(E, t)$ is given by

$$
\begin{aligned}
\phi(E, t) \stackrel{t \ll \lambda}{\approx} \phi_{0}(E)+t \sigma_{\text {tot }}(E)\left[-\phi_{0}(E)\right. \\
\left.+\int_{0}^{1} \frac{\mathrm{d} y}{1-y} \frac{1}{\sigma_{\text {tot }}(E)} \frac{\mathrm{d} \sigma(E /(1-y), y)}{\mathrm{d} y} \phi_{0}\left(\frac{E}{1-y}\right)\right] \\
=\phi_{0}(E)+\frac{t}{\lambda(E)} \phi_{0}(E)\left[-1+\int_{0}^{1} \mathrm{~d} y \Phi(E, y) \eta(E, y)\right]
\end{aligned}
$$

whereas from Eq. 3.46 we have

$$
\phi(E, t) \stackrel{t \ll \lambda}{\approx} \phi_{0}(E)\left(1-t \frac{1-Z(E, t)}{\lambda(E)}\right) .
$$

Comparing Eqs. 3.52 and 3.53 and bearing in mind that they become exact in the limit $t \rightarrow 0$, we may conclude that

$$
\lim _{t \rightarrow 0} Z(E, t)=\int_{0}^{1} \mathrm{~d} y \Phi(E, y) \eta(E, y) .
$$

But taking the limit $t \rightarrow 0$ of Eq. 3.48 yields the same result. Thus the iteration does indeed result in the correct solution for $Z(E, 0)$, and all is well. The accuracies $\rho$ of various examples for $Z$ are given in Sect. 5.2 and Appendix B.2. 


\section{Chapter 4}

\section{Neutrino detection}

\subsection{Neutrino detectors}

The era of detecting neutrinos of extraterrestrial origin began in 1968 with the Homestake experiment [61], which consisted mainly of a tank filled with over 610 tonnes of tetrachloroethene. In very rare cases, an electron neutrino crossing such a tank interacts with one of the chlorine atoms via

$$
{ }_{17}^{37} \mathrm{Cl}+\nu_{e} \longrightarrow{ }_{18}^{37} \mathrm{Ar}+e^{-},
$$

where the neutrino energy must be at least $0.814 \mathrm{MeV}$. The argon atoms thus produced may be extracted by means of sweeping the tank with helium, and as they are unstable, their decay rate is a quantitative measure of their number. Typically, about one neutrino was captured in the Homestake experiment every other day.

Analogously, the reaction

$$
{ }_{31}^{71} \mathrm{Ga}+\nu_{e} \longrightarrow{ }_{32}^{71} \mathrm{Ge}+e^{-}
$$

can be exploited, as has been done by Gallex [21] and the Soviet-American Gallium Experiment (SAGE) [1]. The energy threshold for this reaction lies at $0.236 \mathrm{MeV}$ so that contrary to chlorine-based detectors, gallium-based detectors are able to observe solar neutrinos from the $p p$ chain.

Either of these detectors has a serious drawback, though: Neither angular nor temporal resolution of the detected neutrinos is possible. This situation was improved with water-based detectors consisting of a huge water tank 
surrounded by photomultipliers. Incident neutrinos may undergo electron scattering or initiate an inverse $\beta$-decay,

$$
p+\bar{\nu}_{e} \longrightarrow n+e^{+}
$$

As the scattered electrons and the created positrons move faster than light, they emit Čerenkov radiation (see below), which is detected by the photomultipliers and can be used to obtain some information about the energy and direction of the initial neutrino. This principle has been realized in the Irvine-Michigan-Brookhaven (IMB) detector [36] and in Kamiokande [104] as well as its successor SuperKamiokande [82, 80]. The minimum neutrino energy required for detection is a few $\mathrm{MeV}$.

The Sudbury Neutrino Observatory (SNO) [46] uses heavy water $\left(\mathrm{D}_{2} \mathrm{O}\right)$ instead. Here, the Čerenkov radiation of charged leptons resulting from the reactions

$$
\begin{aligned}
e^{-}+\nu_{l} & \longrightarrow e^{-}+\nu_{l} \\
d+\nu_{e} & \longrightarrow p+p+e^{-} \\
d+\nu_{l} & \longrightarrow n+p+\nu_{l}
\end{aligned}
$$

is employed. (In case of the last reaction, the absorption of the resulting neutron produces a photon, the Compton scattering of which endows an electron with sufficient energy to emit Cerenkov radiation.)

As SNO can detect all neutrino flavors, it played an essential role in establishing flavor oscillation as the explanation of the solar neutrino problem. [5]

When it comes to neutrinos of astrophysical origin (other than those of nearby supernovae), all the detectors mentioned so far are too small. Indeed, one can see from Chapter 5 that presumably a detector volume of about $1 \mathrm{~km}^{3}$ would be required, which obviously is unfeasible for any man-made container.

Hence one resorts to the large volumes of water offered by nature in form of lakes, ice, or the sea. Rather than trying to surround the necessary volume with photomultipliers, they are spread diligently throughout the water (or ice). This idea was first implemented by DUMAND [172] located offshore near the island of Hawaii, and by BAIKAL [19] in the Lake Baikal. At the South Pole, the Antarctic Muon and Neutrino Detector Array (AMANDA) [19, 20] is in operation. AMANDA is currently being extended to IceCube [7], which will constitute the first cubic kilometer detector. A 
similar detector, ANTARES [164], is being developed for the Mediterranean Sea.

All these detectors make use of the fact that neutrinos may undergo charged current neutrino-nucleon interactions in the water,

$$
N+\nu_{l} \longrightarrow X+l
$$

where $X$ and $l$ denote a hadronic rest and the charged lepton corresponding to the initial neutrino, respectively. Under the assumption that the fraction $1-y$ of the initial neutrino energy $E_{\mathrm{i}}$ is transferred to the lepton, the resulting speed $v_{l}$ is given by

$$
v_{l}=c \sqrt{1-\left(\frac{(1-y) E_{\mathrm{i}}}{m_{l} c^{2}}\right)^{-1}}
$$

and as the average inelasticity parameter is of the order of 0.3 (cf. Sect. 2.2), it is evident that for energies $E_{\mathrm{i}} \gg 1 \mathrm{GeV}$, the speed of virtually all leptons exceeds that of light in the detector medium, i. e.

$$
v_{l}>c_{\text {medium }}=\frac{c}{\sqrt{\epsilon(\omega)}}
$$

with the electric permittivity $\epsilon(\omega)$ (in cgs units). By solving the wave equations for a moving point charge in a medium, one may show that the leptons thus emit Cerenkov radiation, the energy of which is given by the FrankTamm equation, [111]

$$
\left(\frac{\mathrm{d} E}{\mathrm{~d} x}\right)_{\mathrm{rad}}=\frac{e^{2}}{c^{2}} \int_{\epsilon(\omega)>1 / \beta^{2}} \omega\left(1-\frac{1}{\beta^{2} \epsilon(\omega)}\right) \mathrm{d} \omega,
$$

where $x$ denotes the distance traversed by the lepton. The Cerenkov light cone thus produced is detected by the photomultipliers interspersed throughout the detector volume and can be used to reconstruct the energy and path of the propagating muon (cf. Fig. 4.1). Here, one has to take into account that the light suffers from absorption and scattering in the detector medium, the amount of which depends on whether the detector is located in a lake, in the sea, or in the polar ice. As a rule of thumb, one may say that the absorption and scattering lengths range from a few dozen to a few hundred meters. Detailed values are given in [64, 127]. 
Due to the extremely short lifetime of the tauon, one cannot expect a detection by its Čerenkov radiation below $E_{i} \lesssim 2 \times 10^{6} \mathrm{GeV}$. However, the hadronic rest $X$ on the right hand side of Eq. 4.1 gives rise to a hadronic cascade, and the tauon decay initiates a second (usually three times larger) particle cascade. [128] The time delay between the two "bangs" must be equal to the distance divided by $c$, and a "double bang" event meeting this criterion can be considered to be reliable smoking gun evidence for a tau neutrino event.

Even though "double bang" events occur for tau neutrinos only, cascades can be initiated by neutrinos of the other flavors as well. [6] The angular distribution of the emitted Čerenkov light differs between hadronic and leptonic cascades. [97]

At ultrahigh energies, the tauon created by inelastic scattering of a tau neutrino skimming the Earth crust or crossing a mountain range may reach the atmosphere before decaying. [76] If so, its decay leads to a shower that might be detected by an air shower telescope like AUGER [51, 23] or gammaray satellites like OWL or EUSO [77].

Another variant of using Cerenkov radiation as a means for neutrino detection exploits the fact that due to the annihilation of positrons and the creation of Compton and $\delta$ electrons [134], showers in a medium have a $10-$ $30 \%$ excess of electrons. They therefore contain a net charge moving at superluminal speed and emit (Čerenkov) radiation in the radio range, which for wavelengths longer than a few centimeters is coherent. [24, 25, 175] This radio signal scales with the square of the energy; its track length increases with the energy and in ice has a value of several kilometers. [198]

The Radio Ice Čerenkov Experiment (RICE), which basically consists of radio dipole receivers deployed together with AMANDA in a $(200 \mathrm{~m})^{3}$ cube [122], was used to establish an upper flux limit for electron neutrinos. This was slightly above the one obtained from AMANDA observations. [121]

Similarly, if a neutrino interacts slightly below the surface of the Moon, a short pulse of radio Čerenkov radiation in the $\mathrm{GHz}$ range may be created, which could be detected by radio telescopes. [17]

A hadronic shower created from a $10^{11} \mathrm{GeV}$ neutrino in water deposits $90 \%$ of its energy on a length scale of $20 \mathrm{~cm}$. From the point of view of ordinary sound this can be considered as an instantaneous and highly localized injection of a heat of about $10 \mathrm{~J}$, which gives rise to an acoustic wave. [126, 129] An experimental verification of the existence of such acoustic signals for traversing proton beams was achieved by means of the accelerators 


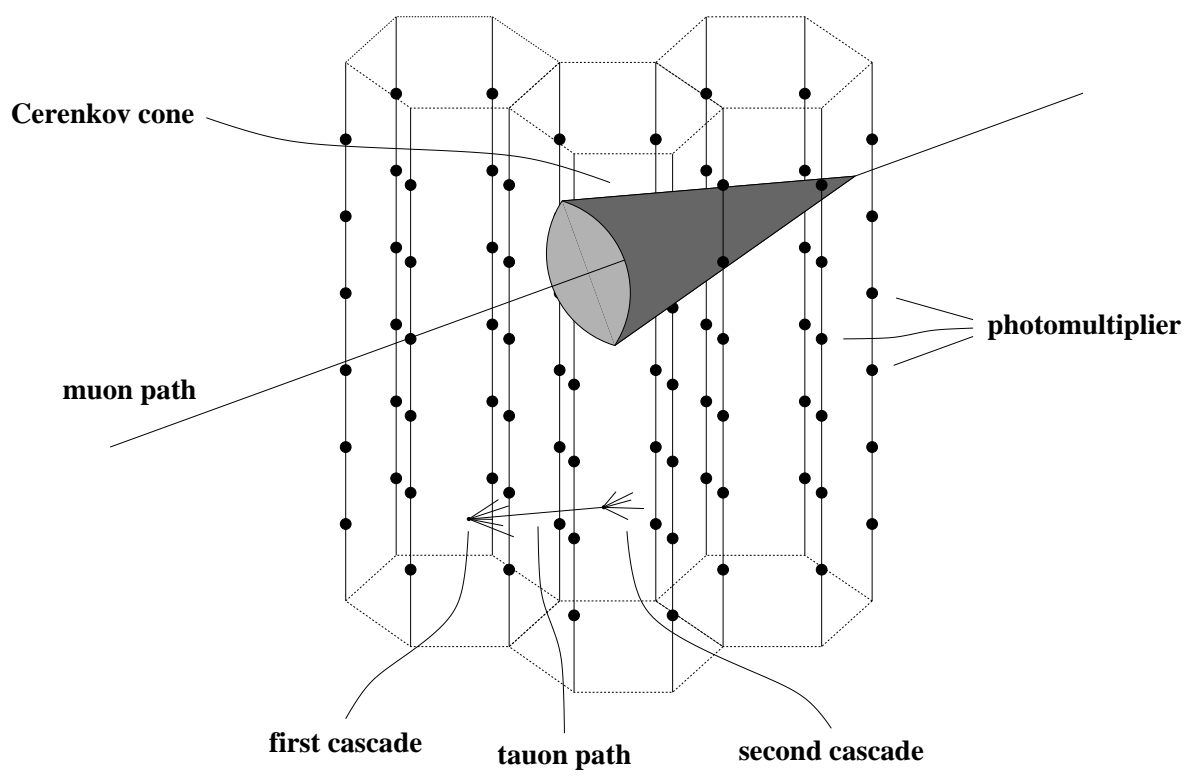

Figure 4.1: Schematic illustration of a water-based neutrino detector. A muon neutrino undergoing inelastic scattering on a nucleon may be turned into a muon, the Cerenkov radiation of which is detected by the photomultipliers. Tau neutrino events can be observed by detecting the "double bang" characteristic for the creation and subsequent decay of a tauon. Not drawn to scale.

at Brookhaven, Harvard, and ITEP. [183, 10] Hence neutrinos in this energy range might be detected by means of an array of hydrophones.

It should be pointed out that this overview over neutrino detection was by no means meant to be exhaustive. The reader is referred to the literature for a discussion of other detectors such as ANITA [148], Fly's Eye [33], AGASA [197], the Telescope Array [176] and GLUE [92], and geological detectors [116].

\subsection{Generic water-based Čerenkov detector}

As should have become clear in the previous section, the sensitivity of a given water-based Čerenkov detector depends both on its design and its location. In general, its calculation requires Monte Carlo simulations and is beyond the scope of this work. Instead, we shall obtain the neutrino event rates for a generic detector, which will be outlined in this section (cf. [99, 101]). 
We assume that the cross section of the detector is the same for all directions and has the value $A_{\operatorname{det}}=1 \mathrm{~km}^{2}$. The volume is taken to be $V_{\operatorname{det}}=1 \mathrm{~km}^{3}$. In addition we assume that every neutrino event occuring in the detector volume $V_{\text {det }}$ actually is detected. Then the corresponding event rate is given by

$$
\frac{\mathrm{d} \dot{N}_{\text {in }}(E, \theta)}{\mathrm{d} \Omega \mathrm{d} E}=V_{\text {det }} Q_{l}(E, \theta)
$$

where the creation rate $Q_{l}(E, \theta)$ of leptons with a given energy $E$ and direction $\theta$ depends on the neutrino flavor considered. For muon neutrinos it has the value

$$
\begin{aligned}
Q_{\mu}(E, \theta)=\int_{E}^{\infty} & \frac{\rho}{m_{p}} \phi_{\nu_{\mu}}\left(E^{\prime}, \theta\right) \frac{\mathrm{d} \sigma_{\mathrm{CC}}\left(E^{\prime}, E\right)}{\mathrm{d} E} \mathrm{~d} E^{\prime} \\
& +\int_{E}^{\infty} \frac{\rho}{m_{p}} \phi_{\nu_{\tau}}\left(E^{\prime}, \theta\right) \frac{\mathrm{d} \sigma_{\mu} \text { from } \tau\left(E^{\prime}, E\right)}{\mathrm{d} E} \mathrm{~d} E^{\prime} .
\end{aligned}
$$

Whereas the first term on the right hand side describes the charged current muon neutrino interactions, the second term accounts for the muons resulting from tauon decays. The cross section of the latter can be written as

$$
\frac{\mathrm{d} \sigma_{\mu \text { from } \tau}(E, z)}{\mathrm{d} z}=\int_{0}^{z} \frac{\mathrm{d} \sigma_{\mathrm{CC}}\left(E, z^{\prime}\right)}{\mathrm{d} z} \frac{1}{z^{\prime}} \frac{\mathrm{d} \Gamma_{\tau \rightarrow \nu_{\tau}+\nu_{\mu}+\mu}\left(z / z^{\prime}\right)}{\mathrm{d} z} \mathrm{~d} z^{\prime} .
$$

Here $z$ and $z^{\prime}$ denote the ratio of the final energy $E_{\mathrm{f}}$ and initial energy $E_{\mathrm{i}}=E$ and that of the final and tauon energy $E_{\tau}$, respectively. The rationale behind this formula is straightforward: For some tauon energy, the differential cross section describes the production of a tauon, and the muon neutrino resulting from the decay of the tauon is accounted for by the distribution $\mathrm{d} \Gamma_{\tau \rightarrow \nu_{\tau}+\nu_{\mu}+\mu} / \mathrm{d} z$. We have to include the factor $1 / z^{\prime}$ in the integrand as (loosely speaking) the differential $\mathrm{d} z$ in $\mathrm{d} \sigma_{\mu}$ from $\tau / \mathrm{d} z$ on the left hand side is equal to $\mathrm{d}\left(E_{\mathrm{i}} / E_{\mathrm{f}}\right)$, whereas the differential $\mathrm{d} z$ in the denominator of $\mathrm{d} \Gamma_{\tau \rightarrow \nu_{\tau}+\nu_{\mu}+\mu} / \mathrm{d} z$ on the right hand side is equal to $\mathrm{d}\left(E_{\mathrm{f}} / E_{\tau}\right)=\mathrm{d}\left(z / z^{\prime}\right)$.

Changing variables in Eq. 4.4 from the energy ratio $z$ to the inelasticity parameter $y=1-z$ yields

$$
\frac{\mathrm{d} \sigma_{\mu \text { from } \tau}(E, y)}{\mathrm{d} y}=\int_{0}^{1-y} \frac{\mathrm{d} \sigma_{\mathrm{CC}}\left(E, 1-z^{\prime}\right)}{\mathrm{d} y} \frac{1}{z^{\prime}} \frac{\mathrm{d} \Gamma_{\tau \rightarrow \nu_{\tau}+\nu_{\mu}+\mu}\left(z / z^{\prime}\right)}{\mathrm{d} z} \mathrm{~d} z^{\prime} .
$$

Concerning the electron neutrino production rate, one would have to add a further term to the analogue of Eq. 4.3, which takes the electron production 
from muon decays into account and is defined completely analogously to Eq. 4.5.

On the other hand, the tau neutrino production rate simply has the value

$$
Q_{\tau}(E, \theta)=\int_{E}^{\infty} \frac{\rho}{m_{p}} \phi_{\nu_{\tau}}\left(E^{\prime}, \theta\right) \frac{\mathrm{d} \sigma_{\mathrm{CC}}\left(E^{\prime}, E\right)}{\mathrm{d} E} \mathrm{~d} E^{\prime}
$$

If a muon created outside the detector in a neutrino-nucleon scattering manages to reach the detector volume, we assume that it is detected. This gives rise to the additional event rate

$$
\frac{\mathrm{d} \dot{N}_{\text {out }}(E, \theta)}{\mathrm{d} \Omega \mathrm{d} E}=\int_{E}^{\infty} A_{\text {det }} Q_{l}\left(E^{\prime}, \theta\right) P_{\text {survival }}\left(E^{\prime}, t_{\text {loss }}\left(E^{\prime}, E\right)\right) \frac{1}{\rho}\left(\frac{\mathrm{d} E_{\mu}\left(E^{\prime}\right)}{\mathrm{d} X}\right)^{-1} \mathrm{~d} E^{\prime} .
$$

Here $\mathrm{d} E_{\mu} / \mathrm{d} X$ consitutes the muon energy loss per column density, which we take to be given by 2.15 . The term $\rho^{-1}\left(\mathrm{~d} E_{\mu}\left(E^{\prime}\right) / \mathrm{d} X\right)^{-1} \mathrm{~d} E^{\prime}$ can be interpreted as the differential path length over which the muon energy changes from $E^{\prime}$ to $E^{\prime}-\mathrm{d} E^{\prime} . t_{\text {loss }}\left(E^{\prime}, E\right)$ denotes the time in which a muon of initial energy $E^{\prime}$ reaches the energy $E$, and the function $P_{\text {survival }}(E, t)$ is defined as the probability that a muon with initial energy $E$ survives at least a time $t$ before decaying. If $\tau_{\mu}$ is the mean lifetime of a muon at rest and if $\gamma$ has its usual meaning from special relativity, $P_{\text {survival }}$ can be obtained as the solution of the differential equation

$$
\frac{\mathrm{d} P_{\text {survival }}\left(E^{\prime}, t\right)}{\mathrm{d} t}=-\frac{1}{\gamma \tau_{\mu}} P_{\text {survival }}\left(E^{\prime}, t\right)=-\frac{1}{\tau_{\mu}}\left(\frac{m_{\mu} c^{2}}{E_{\mu}(t)}\right) P_{\text {survival }}\left(E^{\prime}, t\right)
$$

with the obvious boundary condition $P_{\text {survival }}\left(E^{\prime}, 0\right)=1$. Here $E_{\mu}(t)$ denotes the muon energy at the time $t$ (assuming $E_{\mu}(0)=E^{\prime}$ ), which can be computed from the derivative $\mathrm{d} E_{\mu} / \mathrm{d} X$ and the relation $\mathrm{d} X=\rho c \mathrm{~d} t$.

Concerning electron neutrinos, $\mathrm{d} N_{\text {out }} / \mathrm{d} \Omega \mathrm{d} E$ is obtained completely analogously; one just has to replace the energy loss $\mathrm{d} E_{\mu} / \mathrm{d} X$ for muons by that for electrons. We assume (cf. Eq. 41 of [86])

$$
\frac{\mathrm{d} E_{e}(E)}{\mathrm{d} X}=2 \cdot \frac{62 \mathrm{TeV}}{40 \frac{\mathrm{g}}{\mathrm{cm}^{2}}} \cdot\left(\frac{E}{62 \mathrm{TeV}}\right)^{1 / 2} .
$$

Due to the rapid decay of tauons we ignore the contribution $\mathrm{d} N_{\text {out }} / \mathrm{d} \Omega \mathrm{d} E$ for the tau neutrino event rate. So far, we have assumed that the density is constant. However, for upgoing neutrinos we have to take into account that 
from some depth onwards there is rock rather than water or ice. We do this by including the phenomenological factor $R$ defined in [91],

$$
R(\theta)=\left\{\begin{array}{ll}
0.70+0.48 \cos \theta & \left(\theta \leqslant 95^{\circ}\right) \\
0.70+0.48 \cos \left(95^{\circ}\right) & \left(\theta>95^{\circ}\right)
\end{array},\right.
$$

in our calculations. It should be noted that this factor is less than 1 for downward moving neutrinos, and hence apart from introducing an angular dependence for the effective area, it also diminishes its overall size. While this might be corrected for, we refrain from doing so, as the choice of the exact size has been somewhat arbitrary, anyway. Hence finally we arrive at the formula

$$
\frac{\mathrm{d} \dot{N}(E, \theta)}{\mathrm{d} \Omega \mathrm{d} E}=R(\theta)\left(\frac{\mathrm{d} \dot{N}_{\text {in }}(E, \theta)}{\mathrm{d} \Omega \mathrm{d} E}+\frac{\mathrm{d} \dot{N}_{\text {out }}(E, \theta)}{\mathrm{d} \Omega \mathrm{d} E}\right) .
$$

for the event rate per solid angle and energy in our generic detector. 


\section{Chapter 5}

\section{Astrophysical neutrino sources}

The (proposed) astrophysical neutrino sources can broadly be divided in two classes: On the one hand, there are hadronic sources, in which protons (i.e. hadrons, hence the name) are accelerated. These protons may subsequently interact with photons or with other protons, so that charged pions are created:

$$
\begin{aligned}
& p+\gamma \longrightarrow n+\pi \\
& p+p \longrightarrow p+n+\pi
\end{aligned}
$$

During the decay of the charged pion three neutrinos are produced,

$$
\pi \longrightarrow \mu+\nu_{\mu} \longrightarrow e+\nu_{e}+\nu_{\mu}+\nu_{\mu},
$$

which explains the generic flavor ratio $\nu_{e}: \nu_{\mu}: \nu_{\tau} \approx 1: 2: 0$ for neutrinos of astrophysical origin, if flavor oscillations are disregarded.

It thus should come as no surprise that many sources that are known to involve the acceleration of particles to ultrahigh energies are neutrino source candidates. In the following sections we will consider both galactic and extragalactic examples. Their neutrino fluxes upon reaching Earth (including flavor oscillations where appropriate) are collected in Figs. 5.1 and 5.2 for the case of diffuse and point sources, respectively. Note that explicit values for the corresponding muon and tauon event rates of all the sources covered in this chapter are tabulated in Appendix B.1.

On the other hand, neutrinos might result from the decay of superheavy particles. Again, the neutrino production occurs mainly along the lines of pion decay, so that the generic flavor ratio should hold valid. Such sources are known as non-hadronic. They require non-standard model physics. 
Concerning Figs. 5.1 and 5.2, an obvious caveat might be in order. While the given observational limit from AMANDA strictly speaking applies to a power law spectrum with index -2 only, it may nonetheless serve as a guideline to any source spectrum. Hence one shouldn't take spectra exceeding this limit too serious. For example, a neutrino flux given by the upper MPR flux limit can be ruled out. This should be kept in mind when considering the sources.

\subsection{The Sun}

The most prominent solar neutrino flux arises from nuclear fusion in the core and thus has energies $\ll 1 \mathrm{GeV}$, which is well below the scale considered in this work. However, similar to the terrestrial atmosphere, cosmic ray impingement on the solar atmosphere leads to the production of secondary particles via $p p$ interactions. These subsequently decay, thus producing a flux of high energy electron and muon neutrinos. Following our earlier work [103, 98], in this section we discuss the implications this flux has for tau neutrino observations and the confirmation of neutrino oscillation parameters.

In order to compute this neutrino flux, one first has to evaluate the absorption rate of cosmic rays in the Sun, taking into account the interplanetary and solar magnetic fields, which deflect the protons. [180] The high energy interactions may then be treated by means of Monte Carlo simulations such as JETSET and PYTHIA [181]. Finally, the shadowing effect of inelastic neutrino scattering in the Sun has to be included.

This analysis has been carried out in [108], and its results (which will be used throughout this section) are shown in the left part of Fig. 5.3. For energies below $100 \mathrm{GeV}$ we assume that the solar neutrino flux is given by $\phi(E) \propto E^{-\gamma}$, where $1.75<\gamma<2.45$, thus allowing for some uncertainty due to heliomagnetic effects. The lower limit of $\gamma$ is favored by [180], but the whole range for $\gamma$ is consistent with the EGRET limit on the gamma ray flux of the quiet Sun [186], if a smaller value of $\gamma$ is adopted for energies less than $10 \mathrm{GeV}$.

Now the solar neutrino spectrum may be altered by flavor oscillations during its propagation to Earth, as illustrated in Fig. 5.4. Hence the solar neutrino observations might serve as an independent test of the flavor oscillation parameters obtained by other experiments.

As was discussed in Sect. 2.1, the neutrino flavor oscillations imply that 


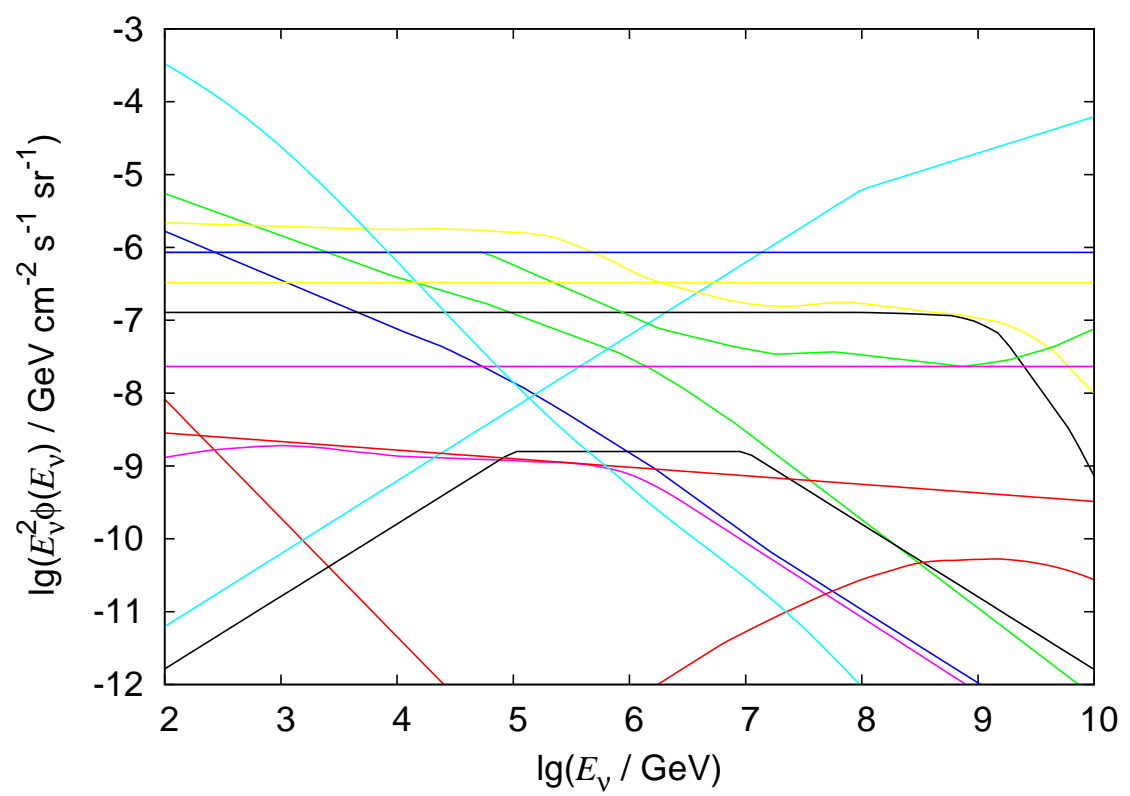

Figure 5.1: Diffuse $\nu_{\mu}+\bar{\nu}_{\mu}$ fluxes at Earth (including flavor oscillations) for various neutrino source candidates. Shown are the fluxes for the Galactic plane [26] (lower (at $10^{4} \mathrm{GeV}$ ) red), cosmic ray interactions in the direction of the Galactic Center (lower (at $10^{5} \mathrm{GeV}$ ) green) and of $b=0^{\circ}, l=45^{\circ}$ [50] (lower (at $10^{5} \mathrm{GeV}$ ) dark blue), proton-nucleon interactions in GRBs [160] (lower (at $100 \mathrm{GeV}$ ) magenta), GRB afterglows [191] (lower (at $100 \mathrm{GeV}$ ) light blue), the limits for GRBlike sources according to [138] (upper (at $100 \mathrm{GeV}$ ) yellow) and [190] (lower (at $100 \mathrm{GeV}$ ) black), proton-photon interaction in AGN jets [137] (lower (at $10^{9} \mathrm{GeV}$ ) red), FR-II galaxies and blazars [35] (upper (at $100 \mathrm{GeV}$ ) black) and galaxy clusters [54] (upper (at 104 GeV) red). In addition, the lower (upper (at $10^{5} \mathrm{GeV}$ ) green) and upper Mannheim-Protheroe-Rachen neutrino flux limit [139] (upper (at $10^{5} \mathrm{GeV}$ ) dark blue) and the Waxman\&Bahcall flux limit [190] (upper (at $100 \mathrm{GeV}$ ) magenta), the atmospheric flux [187] (upper (at $100 \mathrm{GeV}$ ) light blue) and the current AMANDA flux limit [2] (lower (at $100 \mathrm{GeV}$ ) yellow line) are included. More details concerning the sources are found in the following sections. 


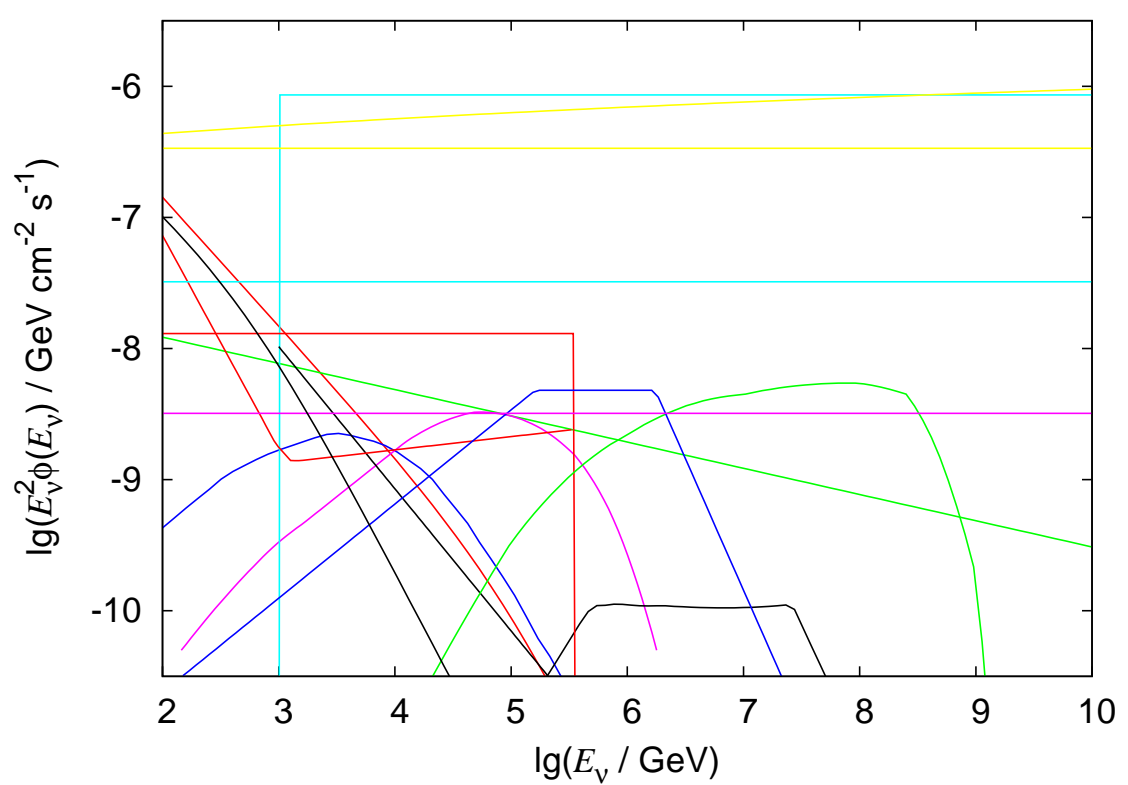

Figure 5.2: $\nu_{\mu}+\bar{\nu}_{\mu}$ fluxes at Earth (including flavor oscillations) for various neutrino point source candidates. Shown are the fluxes for the Sun [108] (top (at $100 \mathrm{GeV}$ ) red), the supernova remnant RX J1713.7-3946 [56] (upper (at 10 ${ }^{5} \mathrm{GeV}$ ) green), the Crab nebula [37] (upper (at $100 \mathrm{GeV}$ ) dark blue), a pulsar wind [152] (lower (at $100 \mathrm{GeV}$ ) magenta), the microquasar SS433 [131, 63] (upper (at 105) light blue), the flare of the magnetar SGR 1806-20 [110] (upper (at $100 \mathrm{GeV}$ ) yellow), the globular cluster Cyg OB2 [18] (upper (at 10 $\mathrm{GeV}$ ) black), the Galactic center [57] (middle (at $100 \mathrm{GeV}$ ) red), the gammma-ray burst GRB941017 [15] (rightmost black), proton-proton interactions in the blazar 3C273 [155, 127] (bottom (at $100 \mathrm{GeV}$ ) red), proton-photon interactions in 3C273 [182] (lower (at 10 $10^{5} \mathrm{GeV}$ ) green), a radio-quiet AGN [16] (lower (at $100 \mathrm{GeV}$ ) dark blue), the blazar 1ES 1959+650 [96] (upper (at $100 \mathrm{GeV}$ ) magenta), and the formation of a massive black hole if the precursor neutron star cluster is formed at the same time [38] (lower (at $10^{5} \mathrm{GeV}$ ) light blue) as and after the envelope from stellar disruptions [39] (lower (at $100 \mathrm{GeV}$ ) yellow). For comparison, the atmospheric flux [187] for a solid angle of $1^{\circ} \times 1^{\circ}$ is included as well (lower (at $10^{5} \mathrm{GeV}$ ) black). For SGR 1806-20 and GRB941017 a fluence rather than flux is relevant; however, this has been "normalized" to the flux given in this plot by dividing through the number of seconds per year. 
the probability for an oscillation from flavor $l$ to $l^{\prime}$ on the neutrino flight to Earth is given by

$$
P_{\nu_{l} \rightarrow \nu_{l^{\prime}}}(E, L)=\left|\delta_{l l^{\prime}}+\sum_{k=2}^{n} U_{l^{\prime} k} U_{l k}^{*}\left[\exp \left(-i \frac{\Delta m_{k 1}^{2} L}{2 E}\right)-1\right]\right|^{2}
$$

with the number $n$ of neutrino flavors and the distance $L$ between Sun and Earth. Because of the high energies, we ignore Mikheyev-SmirnovWolfenstein (MSW) effects (cf. Sect. 2.1), but note that a more precise calculation should take these into account for energies $\ll 100 \mathrm{GeV}$. The mixing matrix $U$ can be written in the form

$$
U=\left(\begin{array}{ccc}
\cos \theta_{\text {Sun }} & \sin \theta_{\text {Sun }} & 0 \\
-\sin \theta_{\text {Sun }} \cos \theta_{\text {atm }} & \cos \theta_{\text {Sun }} \cos \theta_{\text {atm }} & \sin \theta_{\text {atm }} \\
\sin \theta_{\text {Sun }} \sin \theta_{\text {atm }} & -\cos \theta_{\text {Sun }} \sin \theta_{\text {atm }} & \cos \theta_{\text {atm }}
\end{array}\right)
$$

for 3 flavors, where we have used Eq. 2.5 with $\theta_{12} \equiv \theta_{\text {Sun }}, \theta_{23} \equiv \theta_{\text {atm }}$, and $\sin \theta_{13}=0$ (cf. Table 2.2). In case of 4 flavors, i.e. if the existence of a sterile neutrino is assumed, $\mathrm{U}$ may take the form [42]

$$
U=\left(\begin{array}{cccc}
0 & 0 & \cos \theta_{\text {Sun }} & \sin \theta_{\text {Sun }} \\
\cos \theta_{\text {atm }} & \sin \theta_{\text {atm }} & 0 & 0 \\
-\sin \theta_{\text {atm }} & \cos \theta_{\text {atm }} & 0 & 0 \\
0 & 0 & -\sin \theta_{\text {Sun }} & \cos \theta_{\text {Sun }}
\end{array}\right) \text { (case A) }
$$

or

$$
U=\left(\begin{array}{cccc}
\cos \theta_{\text {Sun }} & \sin \theta_{\text {Sun }} & 0 & 0 \\
0 & 0 & \cos \theta_{\text {atm }} & \sin \theta_{\text {atm }} \\
0 & 0 & -\sin \theta_{\text {atm }} & \cos \theta_{\text {atm }} \\
-\sin \theta_{\text {Sun }} & \cos \theta_{\text {Sun }} & 0 & 0
\end{array}\right) \text { (case B). }
$$

Note that in the context of the following discussion the two 4 flavor matrices lead to the same results, as the schemes A and B both consist of a $\nu_{e}-\nu_{\text {sterile }}$ oscillation, which is relevant for the solar neutrino problem, and a $\nu_{\mu}-\nu_{\tau}$ oscillation, which is relevant for the atmospheric neutrino data. Experimental limits for the mass square differences $\Delta m_{k 1} \equiv m_{k}^{2}-m_{1}^{2}$ and the mixing angles $\theta_{\text {atm }}$ and $\theta_{\text {Sun }}$ used in this section are given in Table 5.1. 

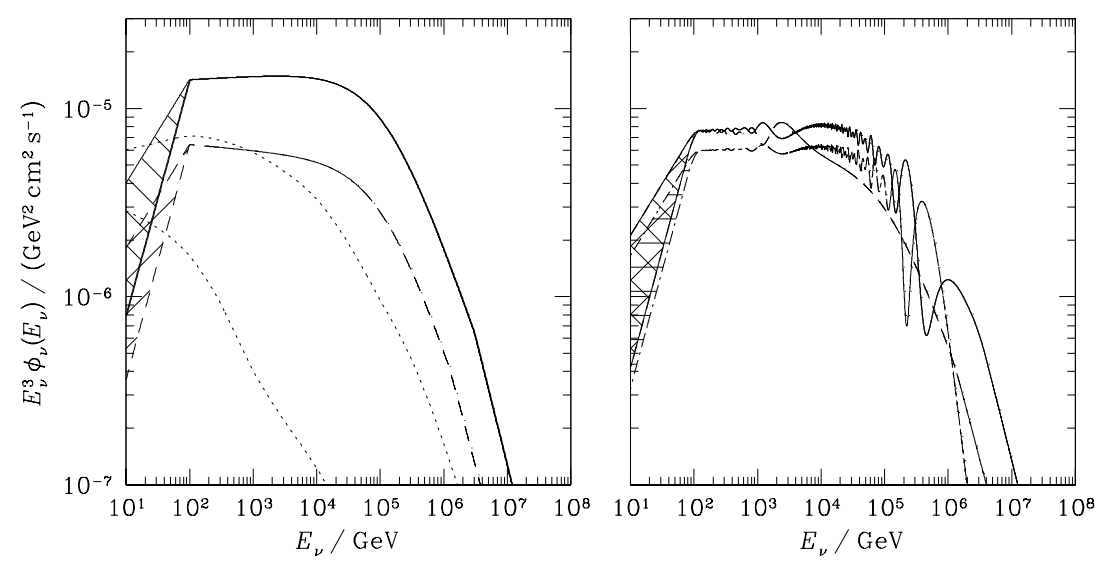

Figure 5.3: Fluxes of solar atmosphere neutrinos (at the earth) for $\nu_{e}$ (dashed), $\nu_{\mu}$ (solid), and $\nu_{\tau}$ (dot-dashed line), integrated over the solid angle of the Sun. Left: The fluxes without neutrino oscillations, as obtained in [108]. For energies lower than $100 \mathrm{GeV} \phi_{\nu} \propto E_{\nu}^{-\gamma}$ is assumed, and the range from $\gamma=1.75$ to 2.45 is shown. In addition, the plot includes the terrestrial atmospheric horizontal $\nu_{e}$ (lower dotted) and $\nu_{\mu}$ flux (upper dotted line), also integrated over the solar disk. [188] Right: The corresponding fluxes for the choice $\Delta m_{\text {Sun }}^{2}=1.9 \times 10^{-5} \mathrm{eV}^{2}$, $\Delta m_{\text {atm }}^{2}=3.5 \times 10^{-3} \mathrm{eV}^{2}, \sin \theta_{\text {Sun }}=0.58$, and $\sin \theta_{\text {atm }}=0.86$, averaged over the interval from $10^{-0.1} E_{\nu}$ to $10^{0.1} E_{\nu}$. This averaging is justified by the limited energy resolution of neutrino telescopes, and it smears out rapid neutrino oscillations.
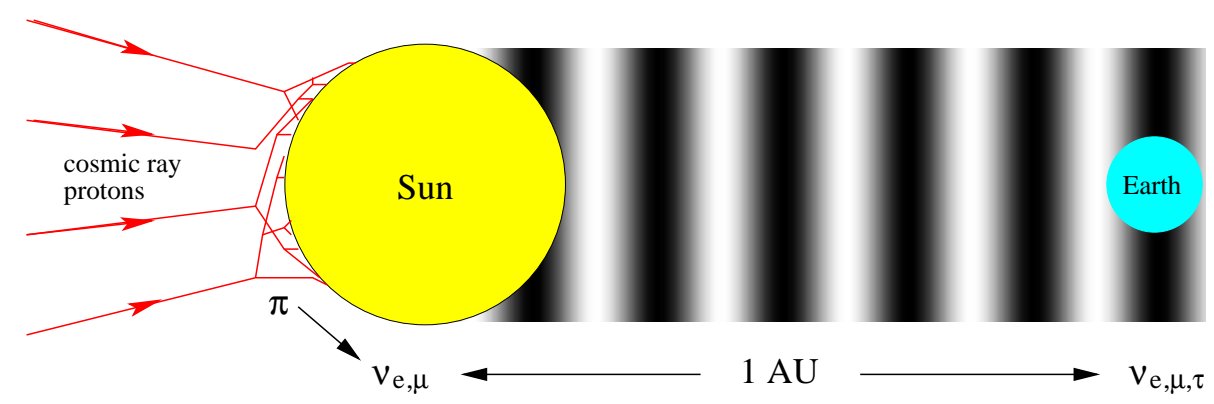

Figure 5.4: Production and propagation of solar atmosphere neutrinos. Cosmic ray interactions in the solar atmosphere lead to the production of secondary particles, which decay into neutrinos. These may undergo flavor oscillations while travelling from their creation site to Earth. Adapted from [127]. 


\begin{tabular}{|l|l|l|}
\hline mixing scheme & mixing angle & mass square difference \\
\hline small mixing MSW & $0.003 \leqslant \sin ^{2} 2 \theta_{\text {Sun }} \leqslant 0.011$ & $4 \times 10^{-6} \mathrm{eV}^{2} \leqslant \Delta m_{21}^{2} \leqslant 1.2 \times 10^{-5} \mathrm{eV}^{2}$ \\
large mixing MSW & $0.42 \leqslant \sin ^{2} 2 \theta_{\text {Sun }} \leqslant 0.74$ & $8 \times 10^{-6} \mathrm{eV}^{2} \leqslant \Delta m_{21}^{2} \leqslant 3.0 \times 10^{-5} \mathrm{eV}^{2}$ \\
vacuum oscillations & $0.70 \leqslant \sin ^{2} 2 \theta_{\text {Sun }} \leqslant 1$ & $6 \times 10^{-11} \mathrm{eV}^{2} \leqslant \Delta m_{21}^{2} \leqslant 1.1 \times 10^{-10} \mathrm{eV}^{2}$ \\
\hline for all mixing schemes: & $0.72 \leqslant \sin ^{2} 2 \theta_{\text {atm }} \leqslant 1$ & $4 \times 10^{-4} \mathrm{eV}^{2} \leqslant \Delta m_{31}^{2} \leqslant 8 \times 10^{-3} \mathrm{eV}^{2}$ \\
\hline
\end{tabular}

Table 5.1: Mixing angles $\theta_{\text {atm }}$ and $\theta_{\text {Sun }}$ and mass square differences $\Delta m_{k 1}^{2}$ for the small mixing angle MSW, the large mixing angle MSW, and the vacuum oscillation solution of the solar neutrino problem, where 3 neutrino flavors are assumed. For 4 neutrino flavors the substitutions $\Delta m_{21}^{2} \rightarrow \Delta m_{43}^{2}, \Delta m_{31}^{2} \rightarrow \Delta m_{21}^{2}$ (case A) or $\Delta m_{31}^{2} \rightarrow \Delta m_{43}^{2}$ (case B) must be made. The limits are taken from [42]. Recent observational data favors the large mixing angle MSW case with slightly different values (see Table 2.2).

Using the transition probability, the neutrino fluxes $\phi^{\text {osc }}$ incident on the Earth can be written as a function of the corresponding fluxes $\phi$ to be expected if there were no flavor oscillations:

$$
\begin{aligned}
& \phi_{e}^{\text {osc }}=\phi_{e} P_{\nu_{e} \rightarrow \nu_{e}}+\phi_{\mu} P_{\nu_{\mu} \rightarrow \nu_{e}} \\
& \phi_{\mu}^{\text {osc }}=\phi_{e} P_{\nu_{e} \rightarrow \nu_{\mu}}+\phi_{\mu} P_{\nu_{\mu} \rightarrow \nu_{\mu}} \\
& \phi_{\tau}^{\text {osc }}=\phi_{e} P_{\nu_{e} \rightarrow \nu_{\tau}}+\phi_{\mu} P_{\nu_{\mu} \rightarrow \nu_{\tau}}
\end{aligned}
$$

The right part of Fig. 5.3 shows an example for fluxes of solar atmosphere neutrinos which undergo oscillations on their way to Earth. Obviously the total event rates $\dot{N}_{\text {tot }}$ in a terrestrial detector depend on the precise form of the flavor oscillations. In order to estimate their value, we use the approximation (cf. Sect. 4.2)

$$
\dot{N}_{\text {tot }}=\int_{E_{0}}^{\infty} \mathrm{d} E \phi(E) \sigma_{\mathrm{CC}}(E) \frac{\rho}{m_{p}} R(E) A
$$

with the detector threshold energy $E_{0}$, the total charged current neutrinonucleon cross section $\sigma_{\mathrm{CC}}$, the density $\rho=1 \mathrm{~g} / \mathrm{cm}^{3}$ of water (or ice), and the effective detector area $A$. $R$ is the mean lepton range or the detector width $h$ in the direction of the Sun, whichever the larger. We assume

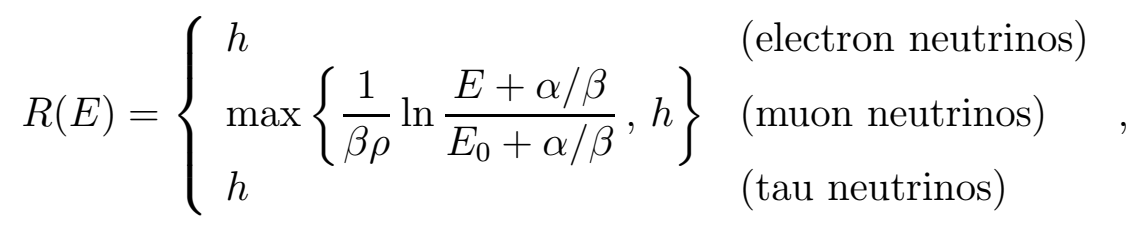


where the values $\alpha=2.5 \mathrm{MeV} /\left(\mathrm{g} \mathrm{cm}^{-2}\right)$ and $\beta=4.0 \times 10^{-6}\left(\mathrm{~g} \mathrm{~cm}^{-2}\right)^{-1}[108]$ are assumed, which differ slightly from those used elsewhere in this work (cf. Sect. 2.4). The contribution of tauon decays to the observed muon event rate has not been included; this should amount to an increase of about $10 \%$. We have employed the CTEQ4DIS parton distributions [125, 58] for the total cross section. In the case of tau neutrinos we had to take into account the phase space limitations due to the large tauon mass.

Choosing the detector values $A=10^{4} \mathrm{~m}^{2}, h=500 \mathrm{~m}$ and $A=1 \mathrm{~km}^{2}$, $h=1 \mathrm{~km}$ with a threshold energy of $E_{0}=10 \mathrm{GeV}$ as an example, the solar atmosphere neutrino fluxes in the left part of Fig. 5.3 yield event rates of $\dot{N}_{e}=0.1-0.2 \mathrm{a}^{-1}, \dot{N}_{\mu}=0.3-0.5 \mathrm{a}^{-1}$ and $\dot{N}_{e}=24-46 \mathrm{a}^{-1}, \dot{N}_{\mu}=46-82 \mathrm{a}^{-1}$, respectively. The range of values reflects the allowed range of $\gamma$ for energies less than $100 \mathrm{GeV}$.

Before turning to the discussion of the oscillation effects, let us briefly consider possible backgrounds. Firstly, the cosmic ray impingement on the terrestrial atmosphere leads to a background in electron and muon neutrinos (cf. Fig. 5.3), which is of the same order as the solar atmosphere neutrino fluxes. For tau neutrinos, however, there is no corresponding background, as virtually no tau neutrinos are created in the atmosphere.

Secondly, the decay of WIMPs in the solar interior might produce a neutrino flux exceeding the one due to cosmic ray interactions. [40]

Finally, there might be an isotropic background from the neutrino sources discussed in the following sections. However, assuming that an upper limit to this background flux is given by the upper MPR flux limit (Sect. 5.5), one obtains even for a cubic kilometer telescope a corresponding event rate of less than one event per year. Shadowing and cascading of neutrinos in the Sun would only further diminish this result. Hence the isotropic background can safely be neglected.

Hence we see that, with the possible exception of WIMP decays, the background is lower or at most comparable to the solar atmosphere flux.

Now let us introduce the ratios

$$
R_{e, \mu} \equiv \frac{\text { total } \nu_{e / \mu} \text { event rate with neutrino oscillations }}{\text { total } \nu_{e / \mu} \text { event rate without neutrino oscillations }}
$$

and

$$
T_{\tau} \equiv \frac{\text { total } \nu_{\tau} \text { event rate with neutrino oscillations }}{\text { total } \nu_{\mu} \text { event rate without neutrino oscillations }}
$$




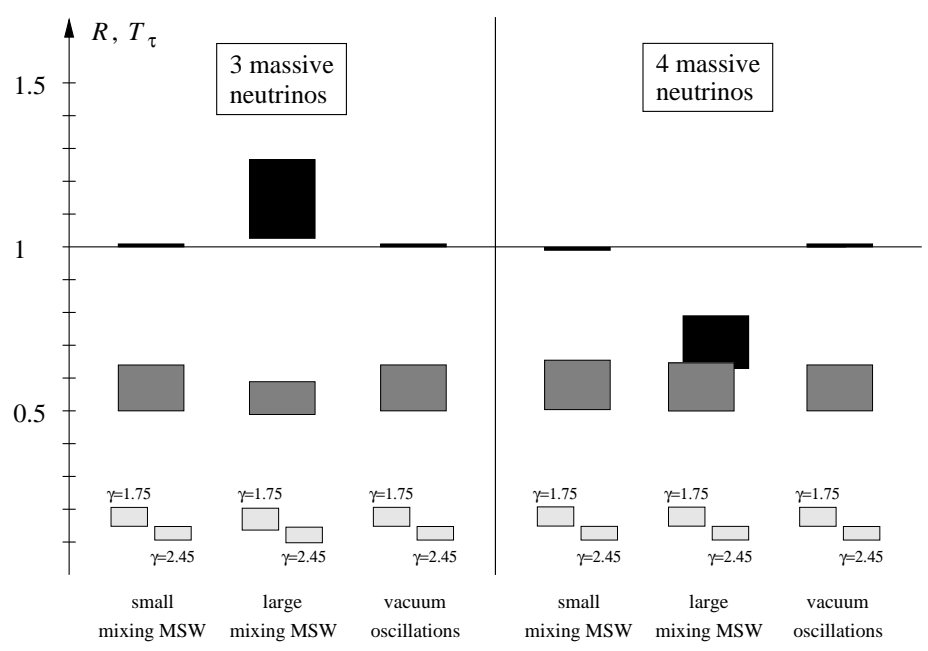

Figure 5.5: $R_{e}$ (black bars), $R_{\mu}$ (dark grey bars), and $T_{\tau}$ (light grey bars) for the various neutrino mixing schemes, if $E_{0}=10 \mathrm{GeV}$ is assumed. $R_{e}$ and $R_{\mu}$ are given for $\gamma=1.75$ (but are virtually the same for any $\gamma$ between 1.75 and 2.45), $T_{\tau}$ for $\gamma=1.75$ and $\gamma=2.45$. The bars show the range of values allowed by the uncertainty of the mixing angle values and the neutrino masses. In case of no neutrino oscillations, $R_{e / \mu}$ and $T_{\tau}$ would be given by $R_{e / \mu}=1$ and $T_{\tau}=0$.

In the denominator of $T_{\tau}$ the muon rather than tau neutrino flux is employed, as the latter is supposed to vanish. Both $R_{e / \mu}$ and $T_{\tau}$ depend on $U, L$, and the $\Delta m_{k 1}^{2}$. However, due to the fact that they involve an integration over energy, the dependence on $L$ and $\Delta m_{k 1}^{2}$ within the mass ranges given in Table 5.1 is weak and can be neglected. Hence $R_{e / \mu}$ and $T_{\tau}$ can be interpreted as an indicator for the form of the mixing matrix.

The ranges of $R_{e / \mu}$ and $T_{\tau}$ for the various mixing matrices considered are given in Fig. 5.5. One sees that both $R_{e}$ and $R_{\mu}$ essentially don't depend on the precise value of $\gamma$ between 1.75 and 2.45. However, because of the limited tauon phase space this isn't true for $T_{\tau}$.

In addition, we infer from Fig. 5.5 that the influence of neutrino oscillations is mostly independent of the mixing scheme, the only exception being the large angle mixing MSW case for electron neutrinos. The event rates for muon and tau neutrinos expected in a cubic kilometer detector are shown in Fig. 5.6 as a function of the threshold energy. For comparison, we note that the rate of tau neutrino events to be expected from the CNGS beam is of the order of 30 per year. [31, 71] 


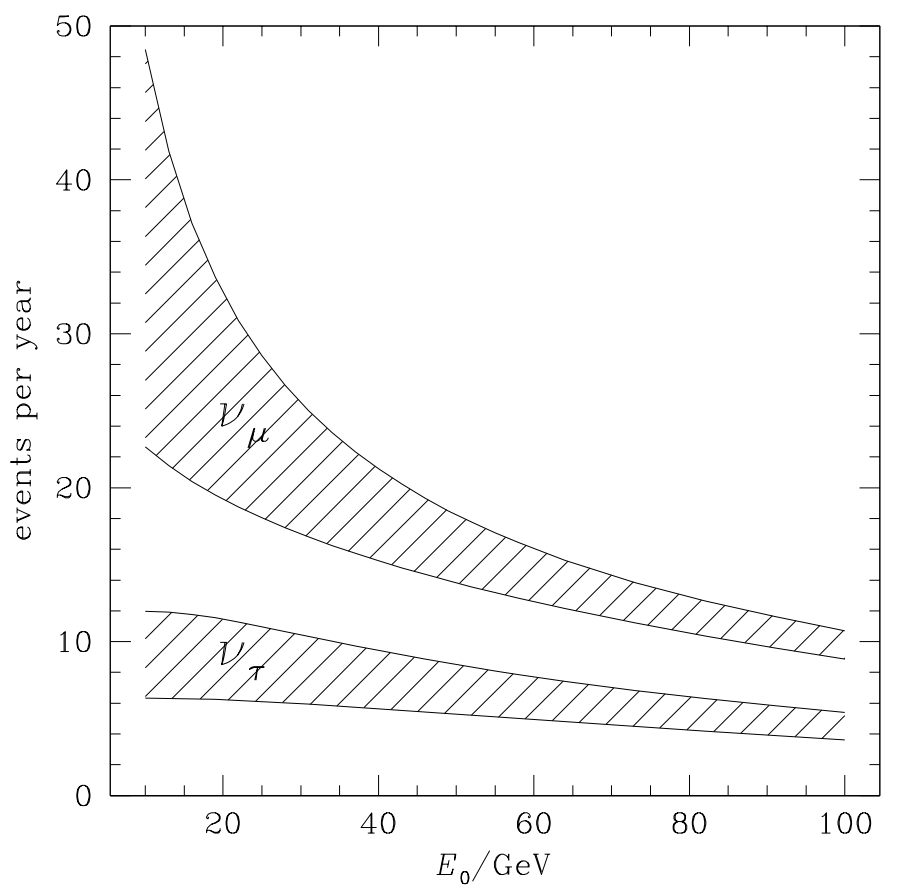

Figure 5.6: $\nu_{\mu}$ and $\nu_{\tau}$ event rates in a $\mathrm{km}^{3}$ detector as a function of the threshold energy $E_{0}$ for the large mixing angle MSW case with three neutrino flavors, taking into account the uncertainty of the initial solar atmosphere neutrino spectrum, the mixing angles and the neutrino masses.

Hence at first sight it seems that, although not producing a sufficiently high event rate in present-day detectors, the Sun should be detectable with next-generation telescopes. But there is a serious drawback: For the energy range considered in this section, the mean angle between directions of an incoming neutrino and the corresponding lepton cannot be neglected. Concerning muon neutrinos it is given by $1.5^{\circ}(E / 100 \mathrm{GeV})^{-0.5}$. Thus effectively the solid angle of the Sun is enlarged, so that the terrestrial background exceeds the solar flux by up to three orders of magnitude for electron and muon neutrinos.

The low angular resolution could be improved if information on the hadronic cascade might be used. Alternatively, one may restrict the neutrino energies to values greater than $100 \mathrm{GeV}$. Then the number of solar atmosphere muon neutrino events would be comparable to the statistical error of the number of background events. 
It should be noted that due to the lack of any significant background, the tau neutrino detectability is not affected by the solid angle over which one has to integrate.

In summary, we may say that the Sun constitutes a guaranteed high energy neutrino source, which might be used for testing the existence of neutrino oscillations on the scale of one astronomical unit for energies well above $1 \mathrm{GeV}$.

\subsection{From source spectra to event rates}

In the preceding chapters we have developed methods for describing the propagation and subsequent detection of neutrinos. Before applying this knowledge to a variety of extrasolar neutrino sources, we shall first discuss a generic source in full detail. For the sake of definiteness, we choose a power law with index -2 as the initial spectrum, assuming the same flux for all flavors. At the end of this section, we will briefly consider the corresponding $Z$ factors for some other initial spectra.

When considering the flux changes due to propagation, a reasonable first step is to obtain the $Z$ factor by means of the method of iteration developed in Sect. 3.4. To this end, the $\mathrm{C}++$ program TEMPEST has been written as part of this work. Its installation and usage is briefly described in Sect. A.1.

Due to the nature of the iteration, it is evident that TEMPEST has to make extensive use of integration, which is accomplished by means of the method gsl_integration_qags provided as part of the GNU Scientific Library [95]. As an implementation of a respective QUADPACK [167] routine, this method applies the 21 point Gauss-Kronrod integration rule (cf. [165]) adaptively, until the absolute and relative errors are below some given limit. The integral value is then calculated by means of an extrapolation using Wynn's epsilon method [193].

The required neutrino-nucleon cross sections are computed with the aid of the Fortran code offered by the CTEQ collaboration for the CTEQ6DIS parton distribution functions, which basically provides an interpolation between a given set of cross section values. [59] The code has been translated into $\mathrm{C}++$ by means of $\mathrm{f} 2 \mathrm{c}[74]$.

Figs. 5.7 and 5.8 illustrate the results obtained for the generic power law spectrum under consideration, showing the $Z$ factor of neutrinos and of antineutrinos at the nadir angles $0^{\circ}, 10^{\circ}, 20^{\circ}, \ldots$, and $90^{\circ}$. Here and in the 


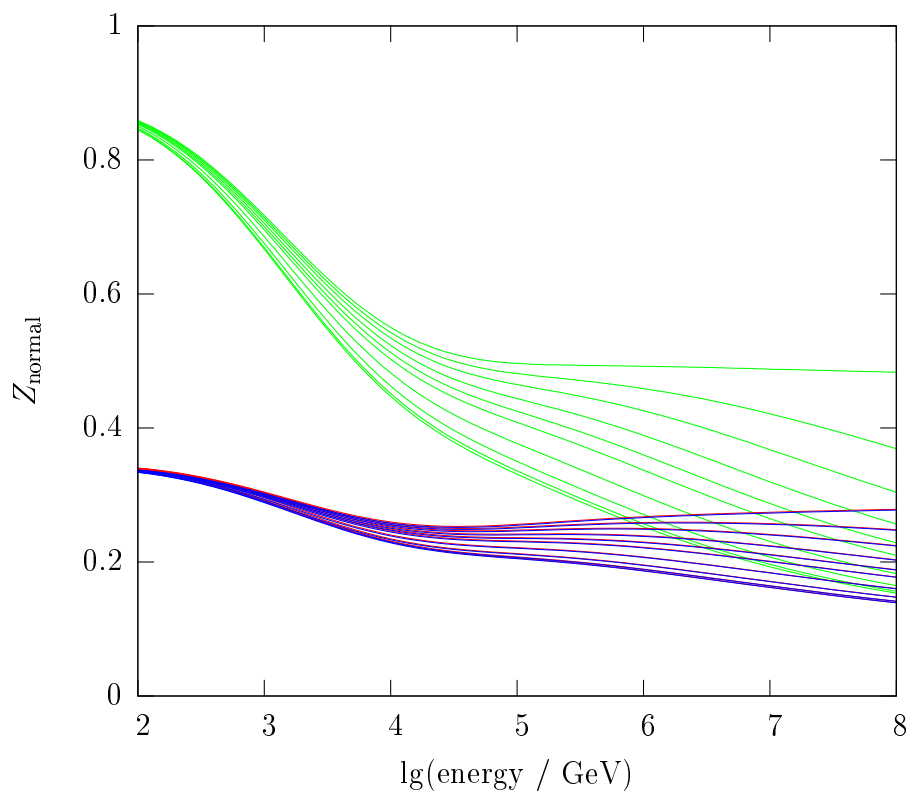

Figure 5.7: $Z$ factor of neutrinos after crossing the Earth at various nadir angles for the generic source introduced in the main text. The nadir angle is increased in steps of $10^{\circ}$ from $0^{\circ}$ (bottom) to $90^{\circ}$ (top lines). Note that the lines for electron and muon neutrinos coincide.

following, we adopt the convention that red (blue, green) lines refer to the electron (muon, tauon) flavor. Five iterations have been carried out in the calculation.

Several things are noteworthy about the $Z$ factors. Firstly, $Z$ is practically the same for electron and muon neutrinos. This is due to the fact that the neutrino-nucleon cross section is the same for both flavors and that the differences between the tauon decay channels leading to the creation of electrons and muons are insignificant.

Secondly, the tau neutrino $Z$ factor exceeds the other $Z$ factors, as here, the regeneration is more effective, leaving the overall tau neutrino number constant (cf. Sect. 3.1).

Thirdly, whereas at sufficiently high energies the $Z$ factors of normal and antineutrinos coincide, they differ from each other at low energies. Noting that up to energies of $\sim 10^{7} \mathrm{GeV}$ both the total cross section and the average inelasticity are smaller for antineutrinos, we can explain this feature qualitatively: There is less regeneration for antineutrinos, but it occurs from 


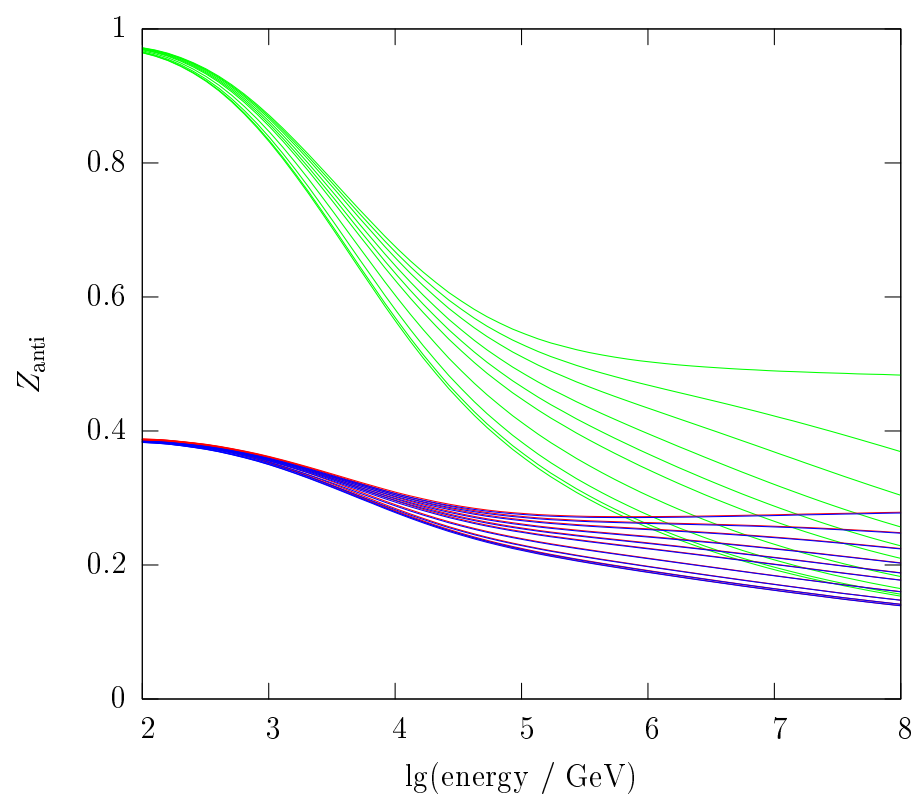

Figure 5.8: The same as the Fig. 5.7, but for antineutrinos.

lower energies and thus with a larger absolute flux.

Having mentioned the (small) differences between normal and antineutrinos, we shall from now on treat the two together.

Turning to the question of the accuracy of the results, one should distinguish between a "numerical" and a "physical" error. The former takes into account that the iteration method isn't perfect. It has been dealt with at length in Sect. 3.4, where it has been shown that some solution for the $Z$ factor can be considered accurate if the ratio $\Delta Z / Z$ (with $\Delta Z$ given by Eq. 3.51) is much less than 1. As can be seen from Fig. 5.9, this condition is fulfilled for our generic source.

The physical error arises from the inaccuracy of the neutrino-nucleon cross sections. If this inaccuracy applies to the total cross section value only (and is taken to be the same for all energies), it is equivalent to an inaccuracy of the column number density. Indeed, if we consider two pairs of cross sections $\mathrm{d} \sigma_{\mathrm{CC} / \mathrm{NC}} / \mathrm{d} y$ and $\mathrm{d} \sigma_{\mathrm{CC} / \mathrm{NC}}^{\prime} / \mathrm{d} y \equiv k \cdot \mathrm{d} \sigma_{\mathrm{CC} / \mathrm{NC}} / \mathrm{d} y$ (where $k$ is some constant), it is easy to see that the corresponding $Z$ factors are related as $Z^{\prime}(E, t)=Z(E, k t)$. Fig. 6.2 can be used to estimate the inaccuracy for nadir angles rather than column number densities.

If, however, the inaccuracy applies to the form rather than absolute value 
of the cross sections, the analysis is more difficult. We estimate the consequences by calculating the $Z$ factor for the "shifted" cross sections

$$
\frac{\mathrm{d} \sigma_{\mathrm{CC} / \mathrm{NC}}^{(s)}(E, y)}{\mathrm{d} y} \equiv \frac{\sigma_{\mathrm{CC} / \mathrm{NC}}(E)}{\sigma_{\mathrm{CC} / \mathrm{NC}}\left(E_{s}\right)} \cdot \frac{\mathrm{d} \sigma_{\mathrm{CC} / \mathrm{NC}}\left(E_{s}, y\right)}{\mathrm{d} y}
$$

and comparing it to the original $Z$ factor. Here, $E_{s}$ is defined by means of the relation

$$
\langle y\rangle_{\mathrm{CC} / \mathrm{NC}}\left(E_{s}\right)=s \cdot\langle y\rangle_{\mathrm{CC} / \mathrm{NC}}(E)
$$

where $\langle y\rangle$ denotes the average inelasticity for an unshifted cross section. Obviously, $E_{s}$ depends both on the shift factor $s$ and the energy $E$. If for some low (high) energy this relation cannot be fulfilled, the minimum (maximum) considered energy is used instead.

It should be evident from Eqs. 5.4 and 5.5 that the total cross section is the same for the shifted and unshifted cross sections, while the average inelasticity of the shifted cross section is in general changed by the factor $s$. Fig. 5.10 shows the ratios of the $Z$ factors obtained for the shifted and the original (unshifted) cross section, using various values for the shift factor and assuming that the entire Earth is crossed.

If the $Z$ factor is known, the corresponding flux at a nadir angle $\theta$ follows directly from the formula (cf. Eq. 3.46)

$$
\phi(E, \theta)=\phi_{0}(E) \exp \left(-\frac{1-Z(E, t(\theta))}{\lambda(E)} t(\theta)\right)
$$

with the initial flux $\phi_{0}$. It is given in Fig. 5.11 for the same nadir angles as in Figs. 5.7 and 5.8.

In accordance with the results for the $Z$ factor, Fig. 5.11 shows that the fluxes for electron and muon neutrinos are practically the same and that they are less than that of tau neutrinos. In addition, we can see from the figure that above $\sim 10^{5} \mathrm{GeV}$ absorption quickly settles in. At $10^{8} \mathrm{GeV}$, the flux of neutrinos crossing the whole Earth is diminished by a factor of $10^{-16}$, and even the flux of neutrinos crossing the Earth at a nadir angle of $80^{\circ}$ is reduced to about one tenth of its original value. Hence it makes sense to disregard energies exceeding $10^{8} \mathrm{GeV}$, and this value is used as the upper energy limit throughout this chapter.

When considering the distance between adjacent lines, one should bear in mind that the difference of the column number densities corresponding to 


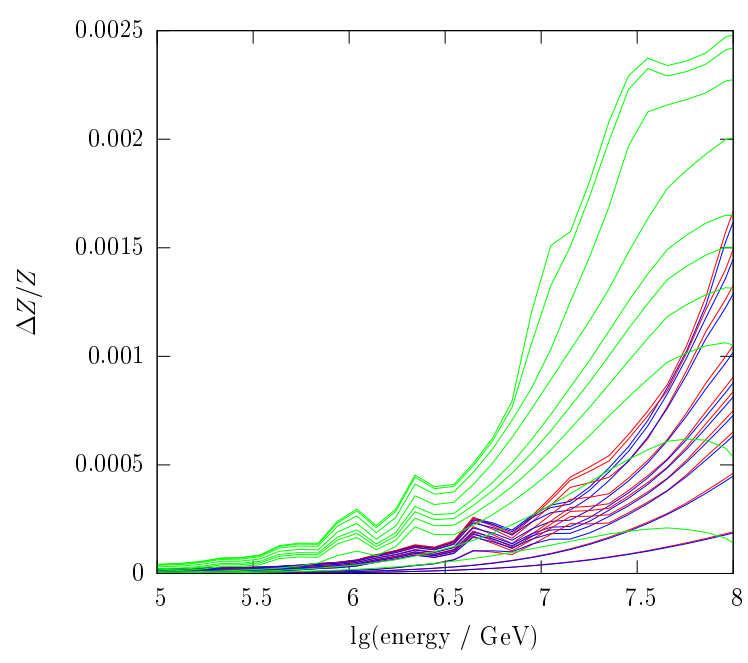

Figure 5.9: Relative inaccuracy of the $Z$ factor (averaged over neutrinos and antineutrinos) due to the numerical imprecision of the iteration method. The inaccuracy is shown for the same nadir angles between $0^{\circ}$ (top) and $90^{\circ}$ (bottom lines) as in Fig. 5.7.

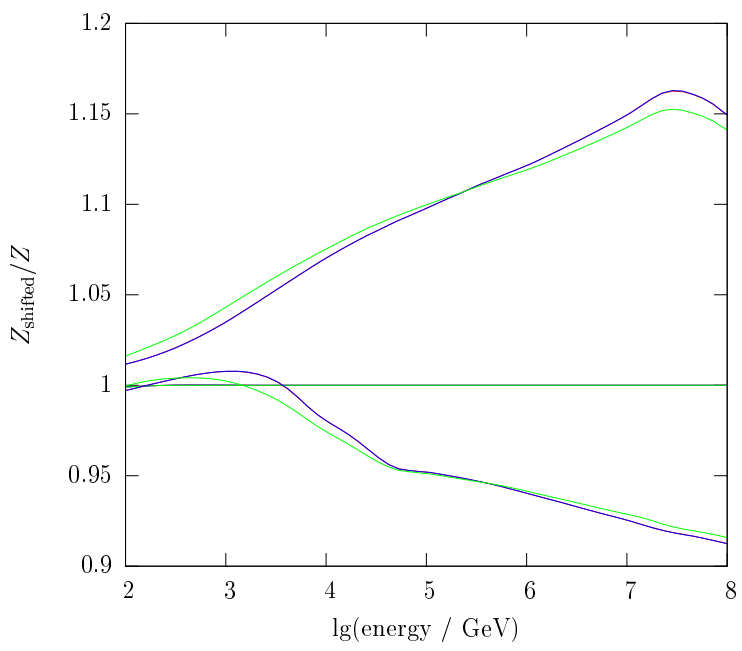

Figure 5.10: Ratio of the $Z$ factors obtained for shifted and unshifted cross sections, assuming a nadir angle of $0^{\circ}$. The lines correspond to a shift factor of 1.1 (upper), 0.8 (lower lines) and 1 (constant line). 


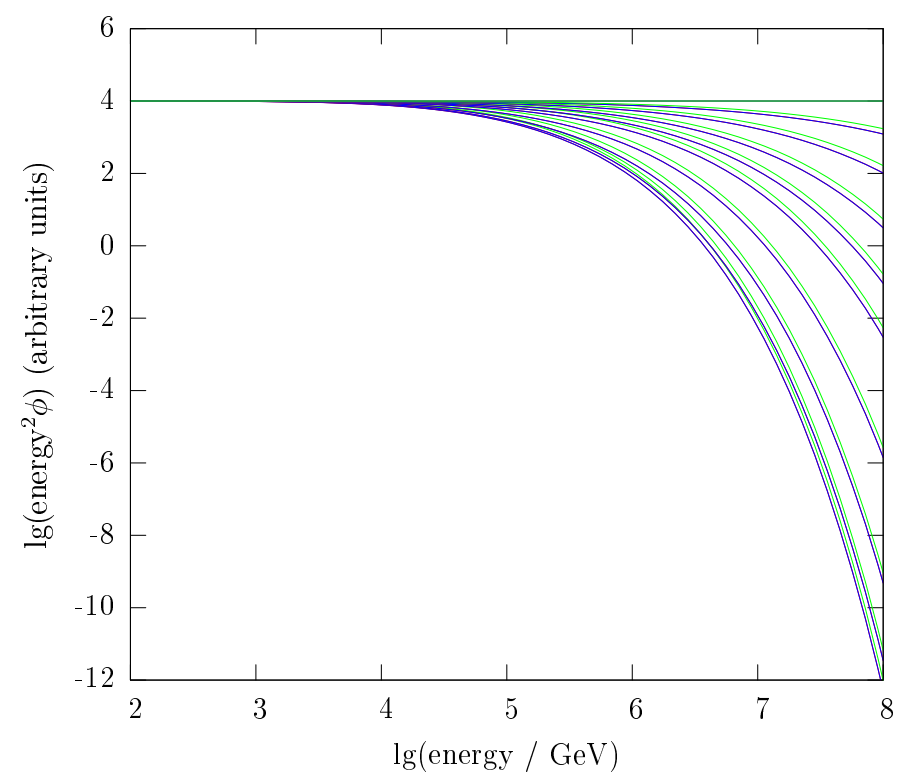

Figure 5.11: Neutrino flux after crossing the Earth at various nadir angles for the generic source. The nadir angles between $0^{\circ}$ (bottom) and $90^{\circ}$ (top lines) correspond to those of Fig. 5.7.

two nadir angles is not proportional to the nadir angle difference. In fact, it is particularly small for nadir angles close to $0^{\circ}$, and this simple geometric effect constitutes the explanation for the close distance between the lines for the nadir angles of $0^{\circ}$ and $10^{\circ}$.

In the following sections, we will use three-dimensional rather than twodimensional plots for the flux as a function of the energy and the nadir angle. The three-dimensional analogue of Fig. 5.11 is given as Fig. 5.12.

Again it is instructive to consider the numerical and physical inaccuracy. This is done in Figs. 5.13 and 5.14, respectively. At first sight, these plots might seem somewhat surprising. After all, how can it be that even though for a nadir angle of $0^{\circ}$, there is a diminution factor of $10^{-16}$, the inaccuracies correspond to a factor of the order of 1 only?

An answer is provided by a back-of-the-evelope calculation, which we perform for the numerical inaccuracy in the case of muon neutrinos. The product of the total cross section and column number density is of the order of 45 for the case considered in the previous paragraph. But from Figs. 5.7 and 5.8 we have that $Z$ is of the order of 0.1 , whereas its relative inaccuracy 


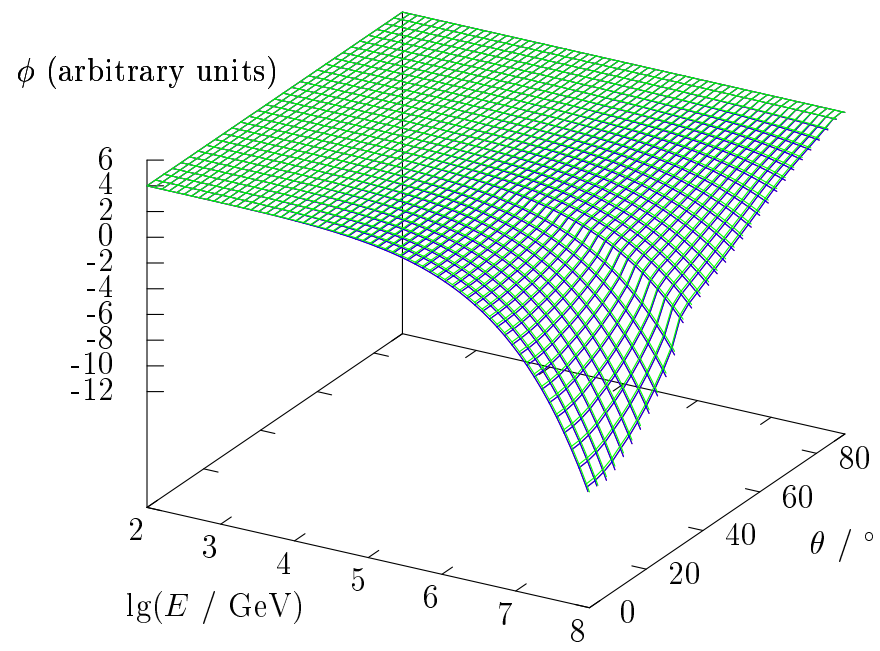

Figure 5.12: Neutrino flux as a function of the energy and the nadir angle.

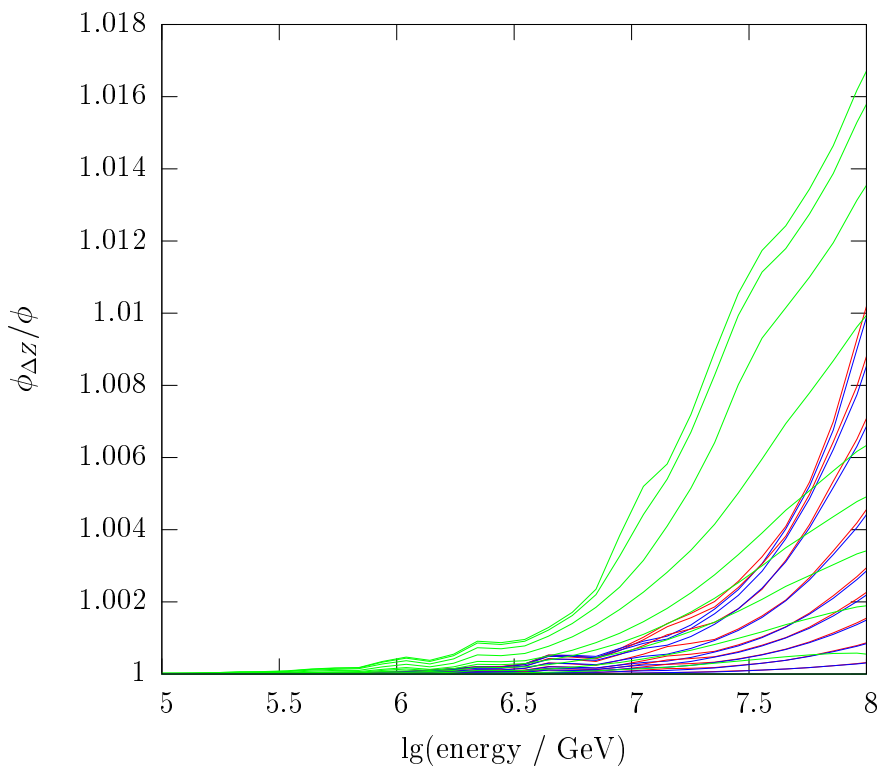

Figure 5.13: Relative inaccuracy of the neutrino flux due to the imprecision of the iteration method for various nadir angles. The nadir angles between $0^{\circ}$ (top lines) and $90^{\circ}$ (energy axis) are the same as in Fig. 5.7. 
can be estimated to be of the order of 0.0015 (from Fig. 5.9). Hence we see that the absolute error $0.0015 \times 0.1 \sim 0.00015$ is small against the column number density and thus has no large impact on the flux changes. Indeed, we have $e^{0.1 \times 0.0015 \times 45} \sim 1.01 \sim 1$ for the inaccuracy factor, in line with our previous result.

If there was just absorption, any neutrino flux would decrease exponentially. Hence the effects of regeneration can be described in terms of the ratio

$$
R(E, \theta) \equiv \frac{\phi[\text { with regeneration }](E, \theta)}{\phi[\text { no regeneration }](E, \theta)}=\frac{\phi(E, \theta)}{\phi_{0}(E) e^{-\sigma_{\text {tot }}(E) t(\theta)}},
$$

which is plotted for the usual nadir angles in Fig. 5.15. $R$ is strictly greater than (or equal) 1 - regeneration may add some flux, but it cannot take away any. It starts to become significant for energies between $10^{4}$ and $10^{5} \mathrm{GeV}$ and may reach values well above 10. Alas, comparing Figs. 5.11 and 5.15, one notes immediately that the importance of regeneration unsurprisingly is correlated with that of absorption, so that the larger the regeneration effects the less flux remains. Still, as we'll see in a moment, regeneration does play a role when considering event rates.

A measure related to the regeneration factor $R$ is the ratio $T$ of the tau and muon neutrino flux. It is shown in Fig. 5.16 for the usual nadir angles. At least for small nadir angles, it differs significantly from 1 for energies as low as $10^{3} \mathrm{GeV}$, and this clearly suggests that for neutrino observations, tau neutrinos are more important than muon neutrinos, because there is a larger flux of them. As will become clear shortly, this notion is somewhat misleading, though.

As for muon neutrinos charged current interactions lead to no regeneration and as almost every fifth regeneration of tau neutrinos via the decay of a tauon yields a muon neutrino as well, one should expect a value of $T \approx 5$ if regeneration was dominated by charged current neutrino-nucleon scattering. However, even at the largest energies considered, $T$ is close to 2 , and hence we reach the conclusion that neutral current must be at least as important as charged current interactions for the regeneration effects.

In order to obtain the event rate corresponding to a given neutrino flux, some choice for a detector has to be made. Here and in the following sections we use the generic detector introduced in Sect. 4.2. A routine for calculating event rates for this detector is included in TEMPEST. 


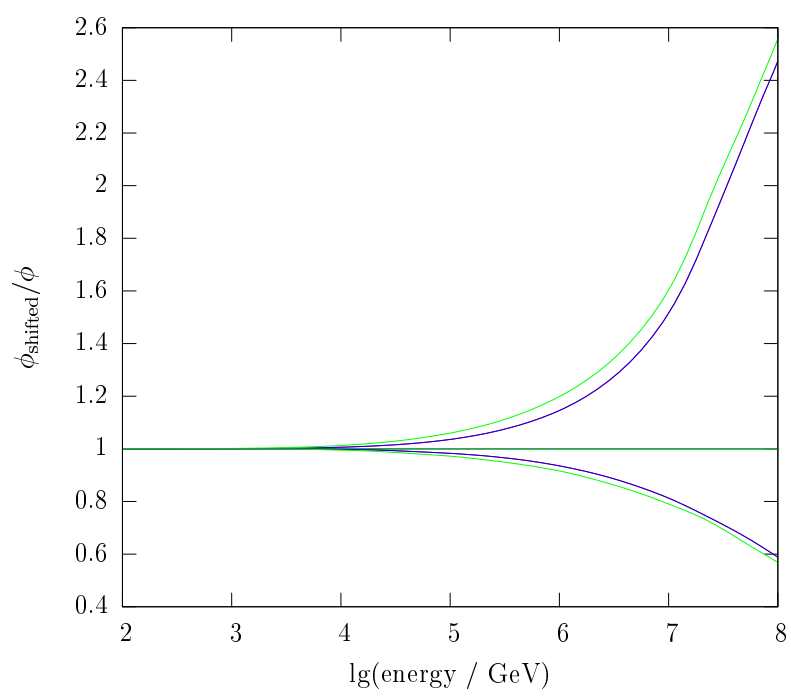

Figure 5.14: Ratio of the neutrino fluxes obtained for shifted and unshifted cross sections, assuming a nadir angle of $0^{\circ}$. The lines correspond to a shift factor of 1.1 (upper), 0.8 (lower lines) and 1 (constant line).

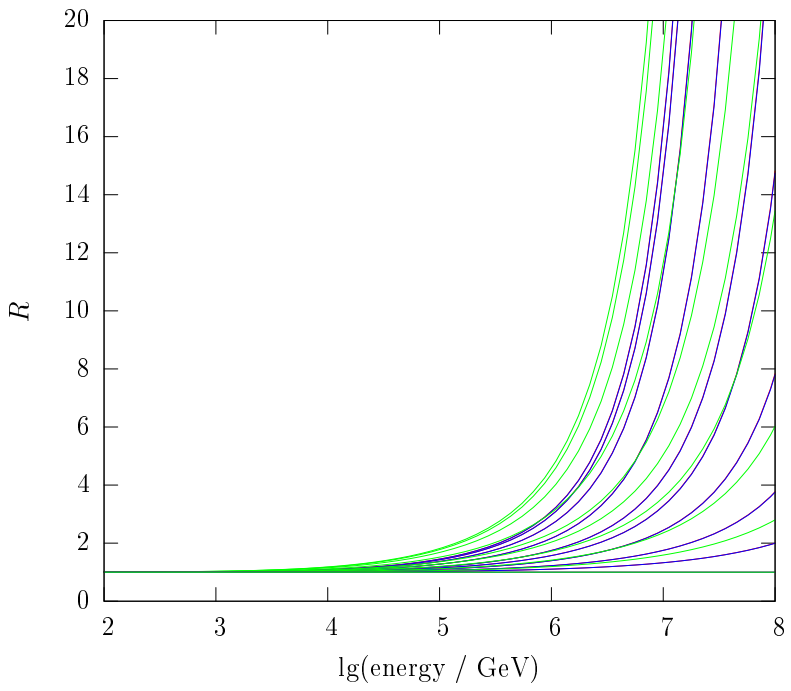

Figure 5.15: Regeneration factor $R$ for a crossing of the Earth at the nadir angles of Fig. 5.7 between $0^{\circ}$ (top) and $90^{\circ}$ (bottom lines). 


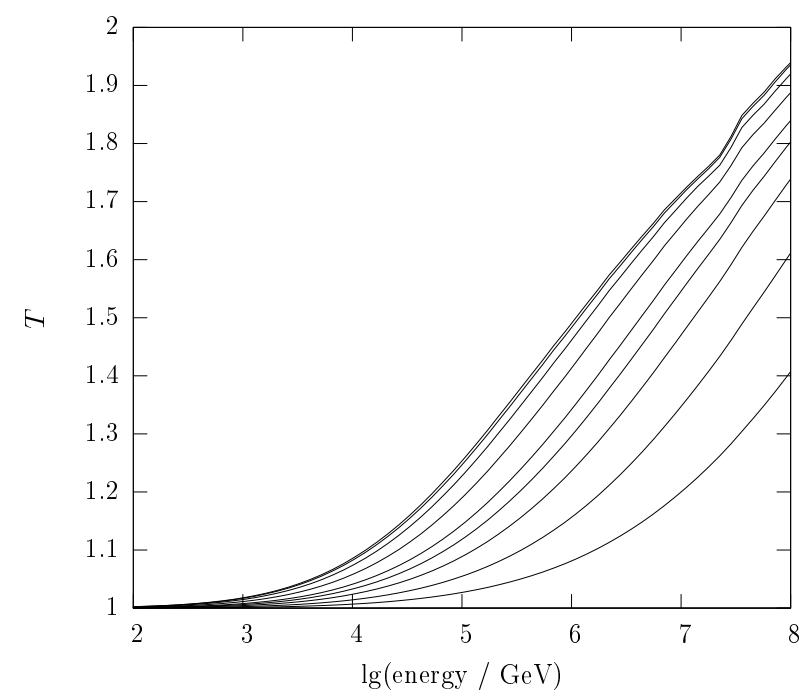

Figure 5.16: Ratio $T$ of the tau and muon neutrino fluxes after crossing the Earth at various nadir angles between $0^{\circ}$ (top line) and $90^{\circ}$ (energy axis). The nadir angles corespond to those of Fig. 5.7. Note that the lines for $0^{\circ}$ and $10^{\circ}$ are nearly indistinguishable.

In this section, we are interested in generic features rather than absolute numbers. Hence we may somewhat arbitrarily normalize all the event rates by dividing them through the value of the differential event rate for an energy of $100 \mathrm{GeV}$ and a nadir angle of $90^{\circ}$. Ignoring the rock correction factor (Eq. 4.6), we then obtain Fig. 5.17 for the differential event rates.

The most striking feature of this plot evidently is the fact that the muon exceeds the tauon event rate by a factor of up to 3 , although the tau neutrino flux is larger than the muon neutrino flux, as we saw above. Hence from the point of view of actual detection, muon neutrinos are more important than tau neutrinos, confirming the caveat voiced above.

The explanation is straightforward. Contrary to the case of tauon neutrinos, there can be non-contained muon events, so that the effective detector volume is significantly larger. In addition (but less importantly), tauon decays contribute to the muon flux inside the detector, hence further increasing the muon event rate.

When obtaining the total from some differential event rate, we have to make some assumption concerning the energy offset below which no detection is made. It is reasonable to expect that the higher this offset, the more 


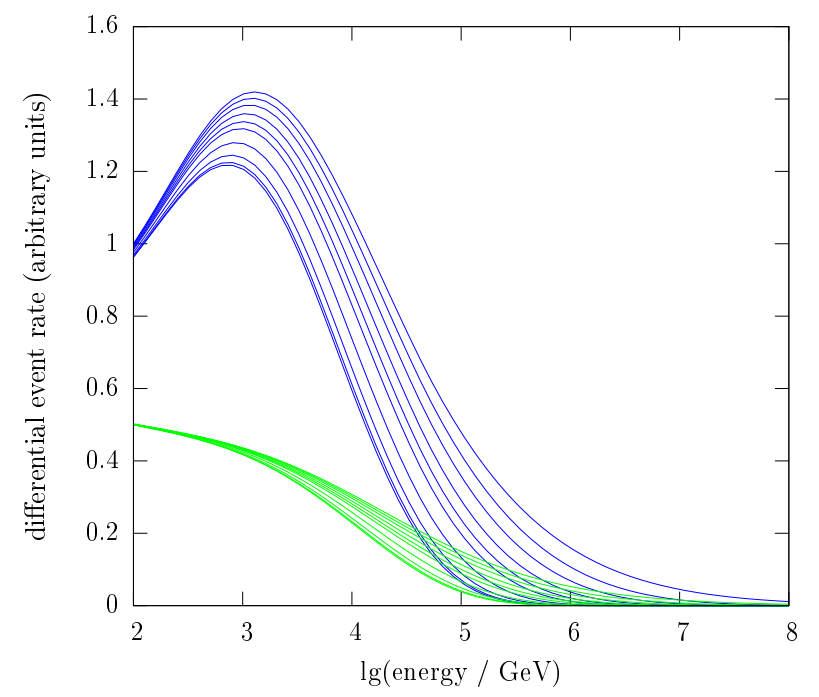

Figure 5.17: Differential event rate for muon and tau neutrinos having crossed the Earth at the various nadir angles between $0^{\circ}$ (bottom) and $90^{\circ}$ (top lines) considered in Fig. 5.7.

dominant absorption and regeneration effects will be.

This expectation is borne out by Fig. 5.18, which shows the total event rate of our generic source as a function of the nadir angle for various energy offsets. Whereas the form is more or less the same for all the lines in this plot, the relative importance of neutrino propagation effects is seen to increase with the energy offset. As the numerical inaccuracy of the neutrino flux is $\lesssim 1 \%$, we can safely say that the numerical inaccuracy of the total event rate must be less than $1 \%$ as well. Estimates of the physical inaccuracy are given in Fig. 5.19.

The most intriguing point to note about Fig. 5.18, however, is the sharp edge between $30^{\circ}$ and $40^{\circ}$. This feature is caused by the density jump at the core-mantle boundary inside the Earth, and hence suggests that neutrino observations might yield some insight into the structure of the inner Earth. We will discuss this idea in full detail in Chapter 6.

In addition, we note that a significant fraction of the events occurs at energies above $10^{4} \mathrm{GeV}$. But in this energy range there is a tau neutrino excess of up to about $10 \%$, and we may conclude that regeneration effects have an influence on the event rate, as claimed above.

Similarly to the case of the neutrino flux, three-dimensional plots will be 


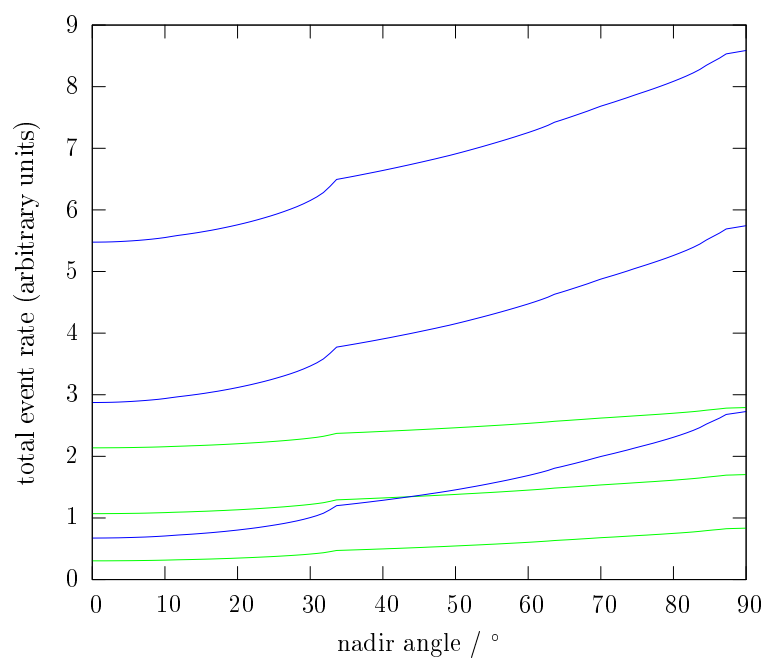

Figure 5.18: Total muon and tauon event rate as a function of the nadir angle for an energy offset of $100 \mathrm{GeV}$ (top), $1000 \mathrm{Gev}$ (middle), and $10^{4} \mathrm{GeV}$ (bottom lines). The same normalization as for the differential event rate is used.

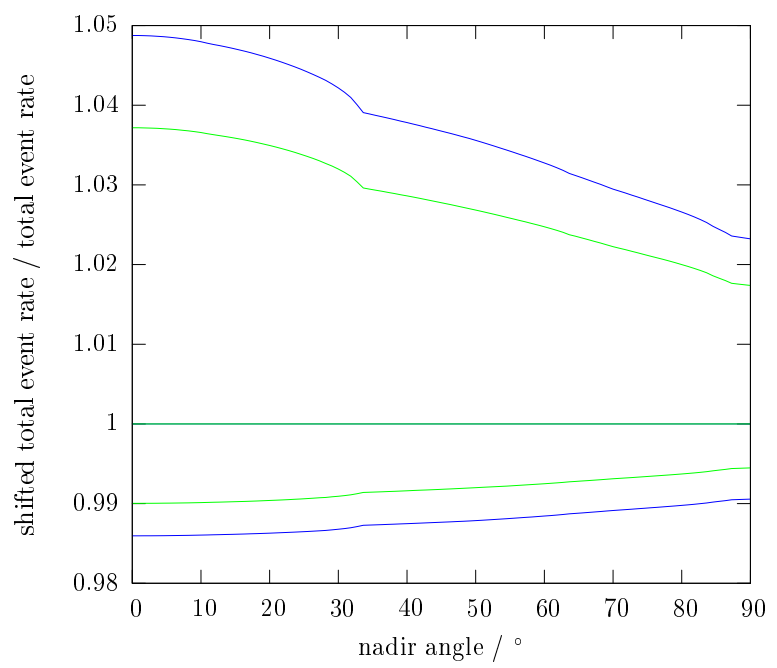

Figure 5.19: Ratio of the total muon and tauon event rates obtained for shifted and unshifted cross sections, assuming an energy offset of $100 \mathrm{GeV}$. The lines correspond to a shift factor of 1.1 (bottom), 0.8 (top lines) and 1 (constant line). 
used in the next sections for the total event rate as a function of the nadir angle and the energy offset. The three-dimensional plot corresponding to Fig. 5.18 is provided for muon events in Fig. 5.20 .

If the flux considered in this section is taken to arise from a point source, the event rate for some nadir angle is to be interpreted as the event rate measured if the source is viewed at that nadir angle. (Of course, in general the nadir angle of a source is a periodic function of time. [56])

On the other hand, if the flux is taken to be diffuse, the event rate must be considered as an event rate per solid angle. But in the foreseeable future, the number of events in all the available detectors will probably be fairly low, and thus a precise determination of event rates per solid angle might be impossible for diffuse fluxes. Then it is a good idea to resort to event rates which are binned with respect to the nadir angle. They can be defined as

$$
\dot{N}\left(\theta_{1}, \theta_{2}\right) \equiv \int_{\Omega\left(\theta_{1}, \theta_{2}\right)} \mathrm{d} \Omega \frac{\mathrm{d} \dot{N}(\theta)}{\mathrm{d} \Omega},
$$

where $\Omega\left(\theta_{1}, \theta_{2}\right)$ is the solid angle for which the nadir angle lies between $\theta_{1}$ and $\theta_{2}$. If we assume that both the initial neutrino spectrum and the Earth are isotropic, the flux cannot depend on the azimuthal angle, and we may rewrite Eq. 5.6 as

$$
\dot{N}\left(\theta_{1}, \theta_{2}\right)=2 \pi \int_{\theta_{1}}^{\theta_{2}} \mathrm{~d} \theta \sin \theta \frac{\mathrm{d} \dot{N}(\theta)}{\mathrm{d} \Omega}=2 \pi \int_{\cos \theta_{2}}^{\cos \theta_{1}} \mathrm{~d}(\cos \theta) \frac{\mathrm{d} \dot{N}(\theta)}{\mathrm{d} \Omega} .
$$

The last expression shows that in order to avoid geometric effects due to a varying size of the solid angle bin, one should use bins with boundaries $\theta_{i}$ which are chosen so that the corresponding values $\cos \theta_{i}$ are equidistant. As should be expected, a plot of a binned event rate for our generic source resembles that of the (unbinned) total event rate. It is given as Fig. 5.21 and ought to be compared to Fig. 5.18.

An analysis similar to that performed so far could be carried out for other sources as well. However, we content ourselves with giving the muon neutrino $Z$ factor for some further generic input spectra. For comparison, we also provide the corresponding $Z$ factors for the case that the contribution of tauons to the neutrino flux is not taken into account.

To be more specific, we consider spectra of the form [153]

$$
\phi_{\alpha, \gamma}(E) \equiv K\left(\frac{E_{0}}{E}\right)^{\gamma+1}\left(1+\frac{E}{E_{0}}\right)^{-\alpha} \xi\left(\frac{E}{E_{\text {cut }}}\right),
$$




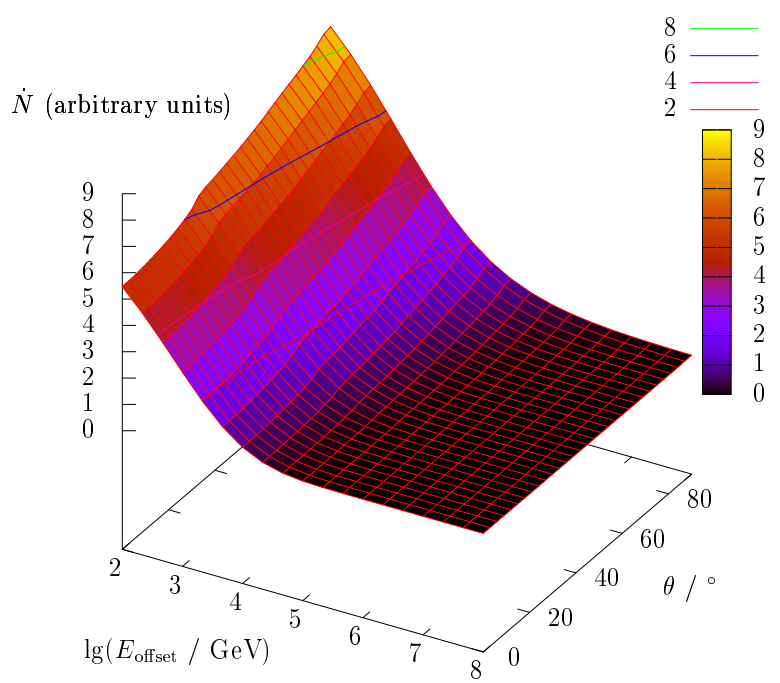

Figure 5.20: Total muon event rate as a function of the nadir angle and the energy offset. The same normalization as for the differential event rate is used.

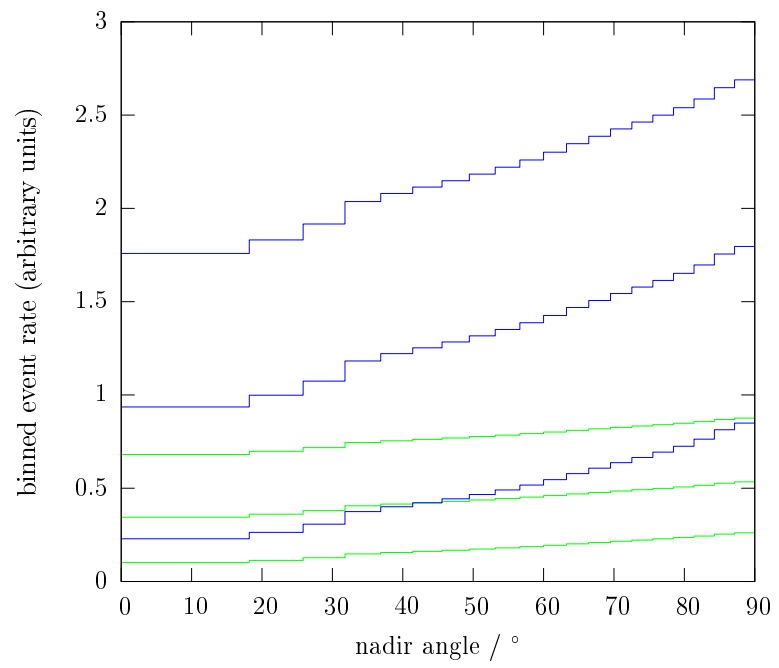

Figure 5.21: Binned muon and tauon event rate for an energy offset of $100 \mathrm{GeV}$ (top), $1000 \mathrm{GeV}$ (middle), and $10^{4} \mathrm{GeV}$ (bottom lines). 

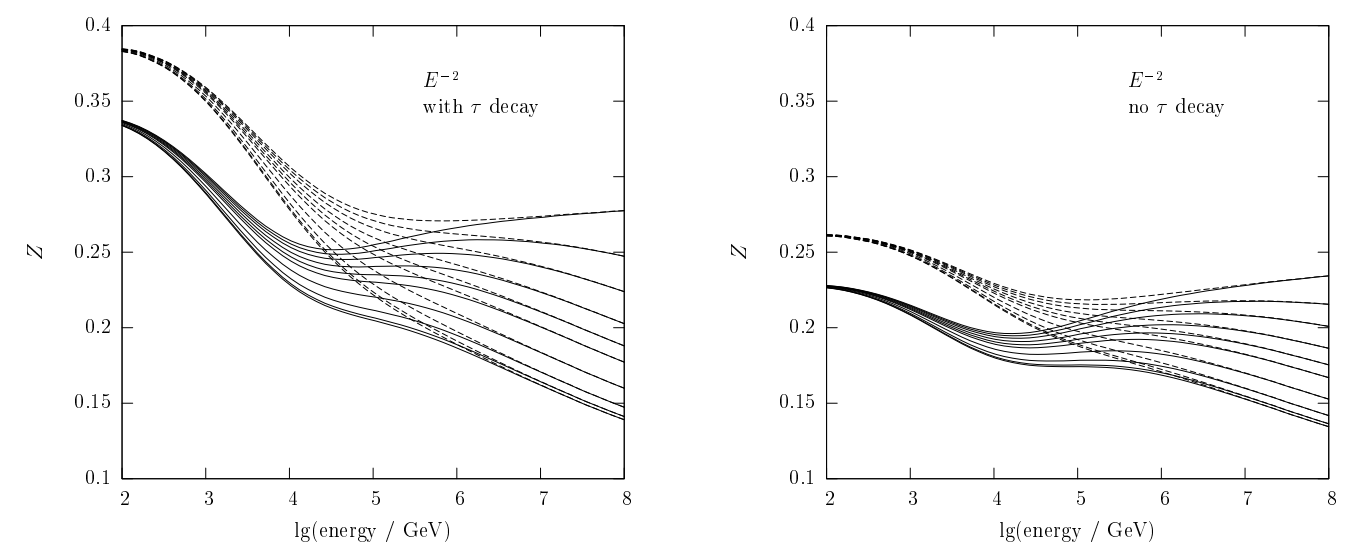

Figure 5.22: Left: $Z$ factor of muon neutrinos (solid) and muon antineutrinos (dashed lines) after crossing the Earth at various nadir angles. A power law with index -2 is assumed as the initial neutrino spectrum. The nadir angle is increased in steps of $10^{\circ}$ from $0^{\circ}$ (bottom) to $90^{\circ}$ (top lines). This plot is equivalent to the muon neutrino curves in Figs. 5.7 and 5.8, Right: The same as the left figure, but under the assumption that there is no contribution to the neutrino flux from tauon decays.

where $E_{0}$ and $E_{\text {cut }}$ have the values $E_{0} \equiv 10^{6} \mathrm{GeV}$ and $E_{\text {cut }} \equiv 3 \times 10^{10} \mathrm{GeV}$, respectively. As a constant factor in an input spectrum doesn't influence the value of $Z$, an arbitrary value may be chosen for the constant $K$. Finally, the function $\xi$ serves as a cut-off and can be defined as

$$
\xi(x) \equiv\left\{\begin{array}{ll}
1 /(1+\tan (\pi x / 2)) & (x<1) \\
0 & (\text { otherwise })
\end{array} .\right.
$$

For convenience, let us denote a spectrum of the form Eq. 5.7 with the parameter values $\alpha=\alpha^{\prime}$ and $\gamma=\gamma^{\prime}$ as $\operatorname{Gen}\left(\alpha^{\prime}, \gamma^{\prime}\right)$. Then the $Z$ factors of a power law with index -2 , the lower MPR flux limit, $\operatorname{Gen}(0.5,1.0)$, Gen(0.5,1.5), $\operatorname{Gen}(1.0,0.5)$, and $\operatorname{Gen}(1.0,2.0)$ are given in Figs. 5.22 5.27, and the corresponding numerical inaccuracies are summarized in Table 5.2.

One should note several points. Firstly, the spectral form of the $Z$ factor depends crucially on the initial neutrino spectrum. Of course this just reflects the fact that a non-zero $Z$ factor requires regeneration, the amount of which decreases with a steepening of the neutrino spectrum, as can be seen from, say, Figs. 5.26 and 5.27 .

Secondly, the $Z$ factor for antineutrinos exceeds that for neutrinos at 

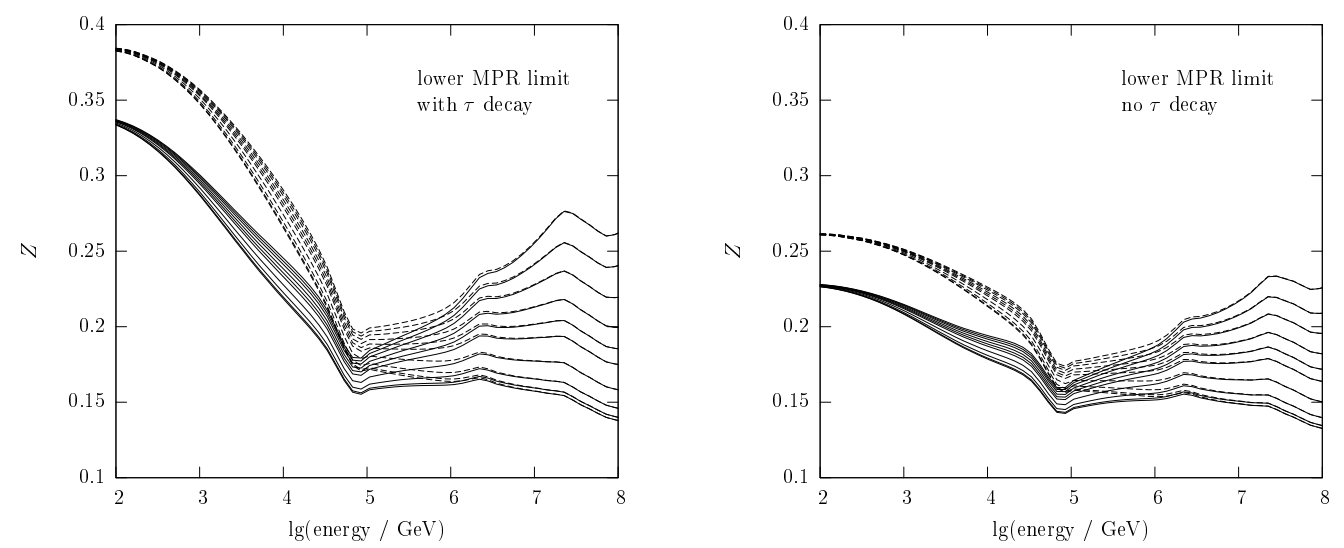

Figure 5.23: Left: $Z$ factor of muon neutrinos (solid) and muon antineutrinos (dashed lines) after crossing the Earth at various nadir angles. The lower MPR flux limit is assumed as the initial neutrino spectrum. The nadir angle is increased in steps of $10^{\circ}$ from $0^{\circ}$ (bottom) to $90^{\circ}$ (top lines). Right: The same as the left figure, but under the assumption that there is no contribution to the neutrino flux from tauon decays.
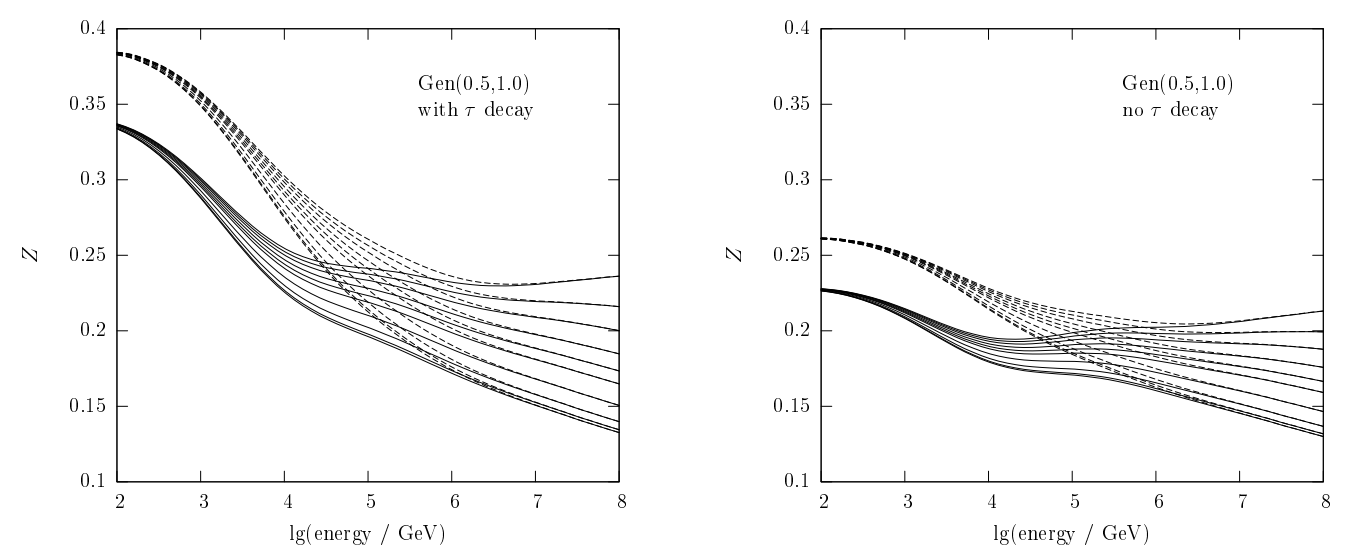

Figure 5.24: Left: $Z$ factor of muon neutrinos (solid) and muon antineutrinos (dashed lines) after crossing the Earth at various nadir angles. $\operatorname{Gen}(0.5,1.0)$ is assumed as the initial neutrino spectrum. The nadir angle is increased in steps of $10^{\circ}$ from $0^{\circ}$ (bottom) to $90^{\circ}$ (top lines). Right: The same as the left figure, but under the assumption that there is no contribution to the neutrino flux from tauon decays. 

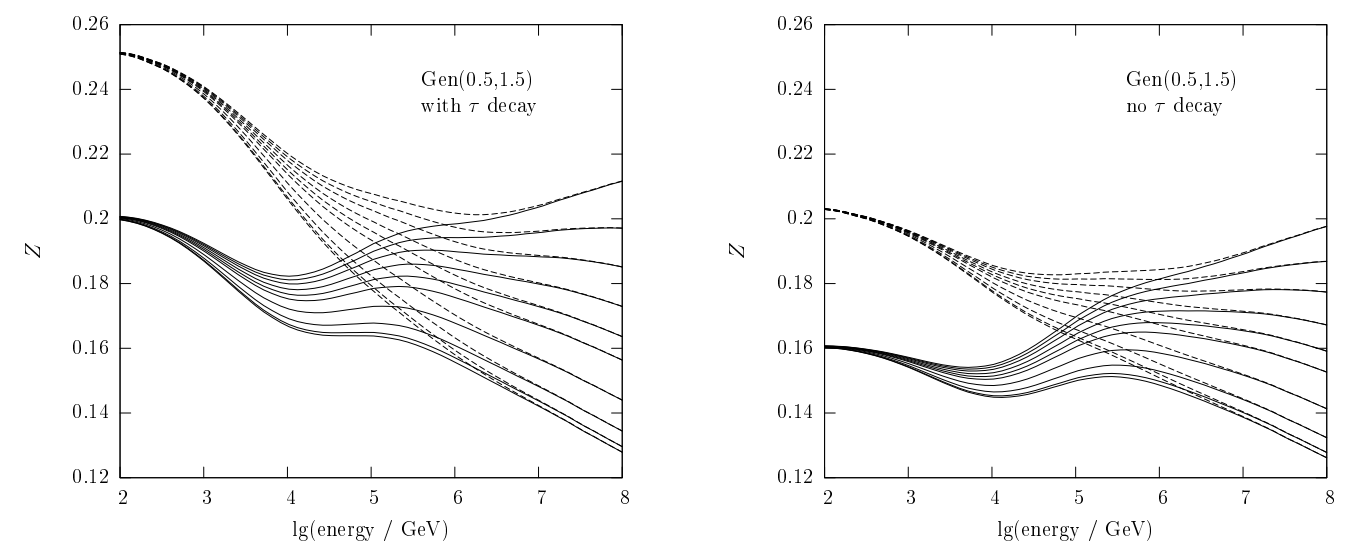

Figure 5.25: Left: $Z$ factor of muon neutrinos (solid) and muon antineutrinos (dashed lines) after crossing the Earth at various nadir angles. Gen $(0.5,1.5)$ is assumed as the initial neutrino spectrum. The nadir angle is increased in steps of $10^{\circ}$ from $0^{\circ}$ (bottom) to $90^{\circ}$ (top lines). Right: The same as the left figure, but under the assumption that there is no contribution to the neutrino flux from tauon decays.
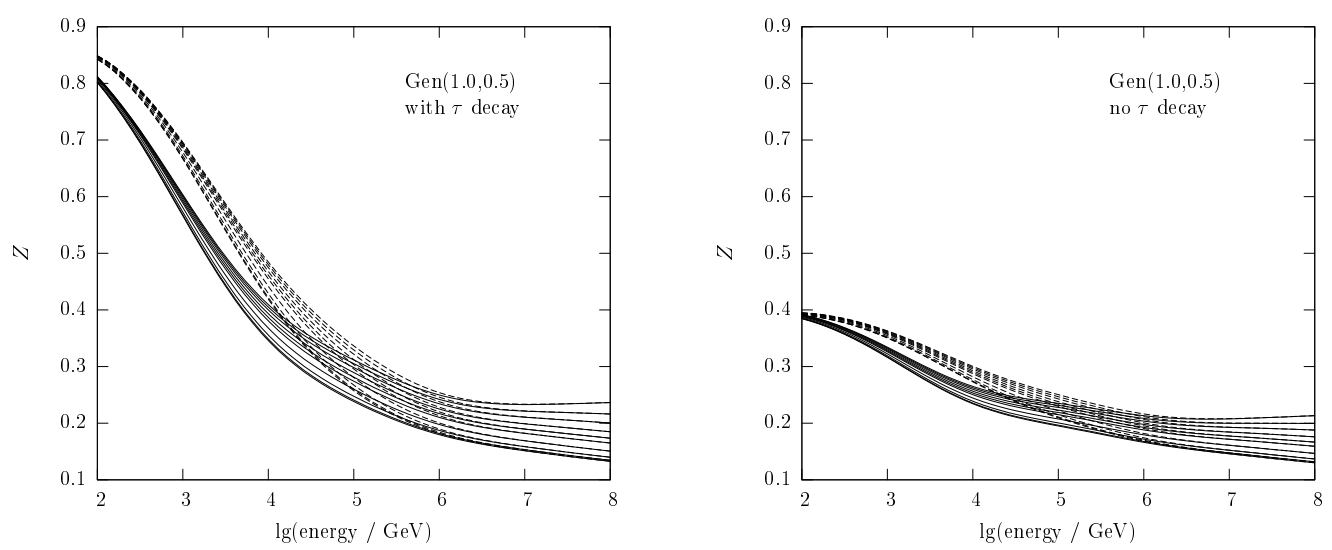

Figure 5.26: Left: $Z$ factor of muon neutrinos (solid) and muon antineutrinos (dashed lines) after crossing the Earth at various nadir angles. Gen(1.0,0.5) is assumed as the initial neutrino spectrum. The nadir angle is increased in steps of $10^{\circ}$ from $0^{\circ}$ (bottom) to $90^{\circ}$ (top lines). Right: The same as the left figure, but under the assumption that there is no contribution to the neutrino flux from tauon decays. 

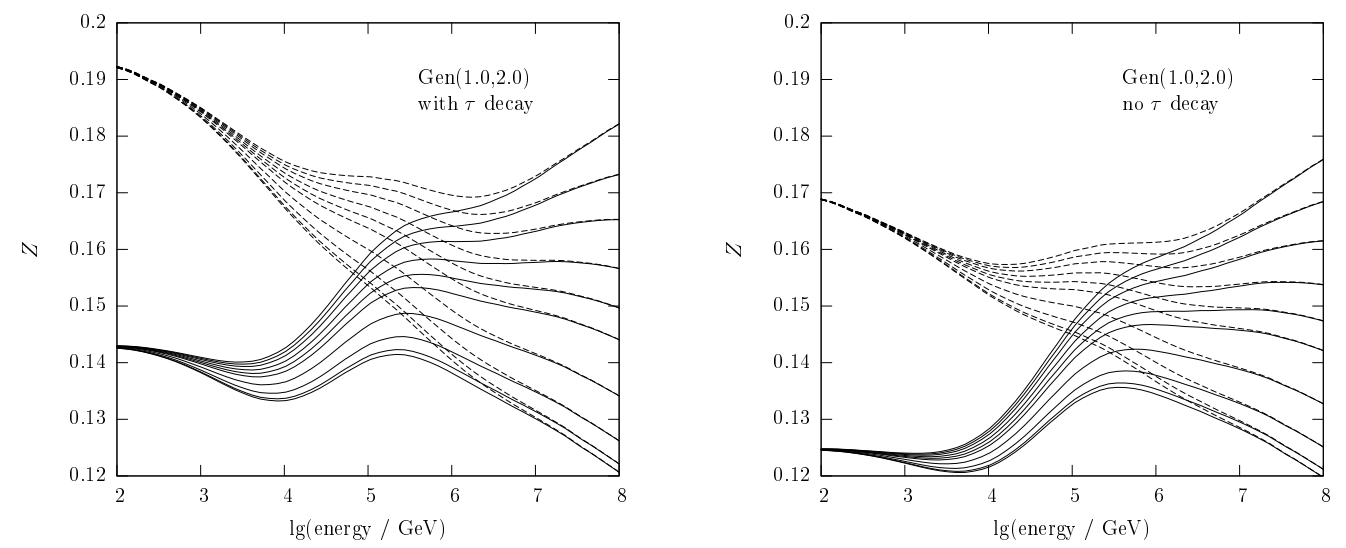

Figure 5.27: Left: $Z$ factor of muon neutrinos (solid) and muon antineutrinos (dashed lines) after crossing the Earth at various nadir angles. Gen(1.0,2.0) is assumed as the initial neutrino spectrum. The nadir angle is increased in steps of $10^{\circ}$ from $0^{\circ}$ (bottom) to $90^{\circ}$ (top lines). Right: The same as the left figure, but under the assumption that there is no contribution to the neutrino flux from tauon decays.

\begin{tabular}{|l|c|llll|}
\hline spectrum & flavor & \multicolumn{4}{|c|}{ energy } \\
& & \multicolumn{1}{|c|}{$\mathbf{1 0}^{\mathbf{2}}$} & $\mathbf{1 0}^{\mathbf{4}}$ & \multicolumn{1}{c|}{$\mathbf{1 0}^{\mathbf{6}}$} & \multicolumn{1}{c|}{$\mathbf{1 0}^{\mathbf{8}}$} \\
\hline lower MPR limit & $\nu_{\mu}$ & $1.5 \times 10^{-6}$ & $9.3 \times 10^{-7}$ & $8.5 \times 10^{-5}$ & 0.0043 \\
upper MPR limit & $\nu_{\mu}$ & $1.9 \times 10^{-6}$ & $8.5 \times 10^{-6}$ & $5.5 \times 10^{-5}$ & 0.0017 \\
Gen(0.5,1.0) & $\nu_{\mu}$ & $1.5 \times 10^{-6}$ & $1.2 \times 10^{-6}$ & $5 \times 10^{-6}$ & 0.00015 \\
Gen(0.5,1.5) & $\nu_{\mu}$ & $7.9 \times 10^{-7}$ & $3 \times 10^{-7}$ & $7.4 \times 10^{-7}$ & $8.1 \times 10^{-5}$ \\
Gen(1.0,0.5) & $\nu_{\mu}$ & $6.3 \times 10^{-6}$ & $4 \times 10^{-6}$ & $6 \times 10^{-6}$ & 0.00015 \\
Gen(1.0,2.0) & $\nu_{\mu}$ & $1.4 \times 10^{-6}$ & $1.5 \times 10^{-7}$ & $3 \times 10^{-7}$ & $3.5 \times 10^{-5}$ \\
Gen(1.0,0.5) & $\nu_{\tau}$ & 0.0001 & $6.4 \times 10^{-6}$ & $2.1 \times 10^{-5}$ & 0.0012 \\
Gen(1.0,2.0) & $\nu_{\tau}$ & $8.6 \times 10^{-6}$ & $2.9 \times 10^{-7}$ & $1.2 \times 10^{-6}$ & 0.00019 \\
\hline
\end{tabular}

Table 5.2: Numerical inaccuracies $\Delta Z / Z$ for various energies, assuming that the Earth has been crossed at a nadir angle of $0^{\circ}$. The inaccuracies are given for the initial spectra covered by Figs. $5.22-5.27$. 

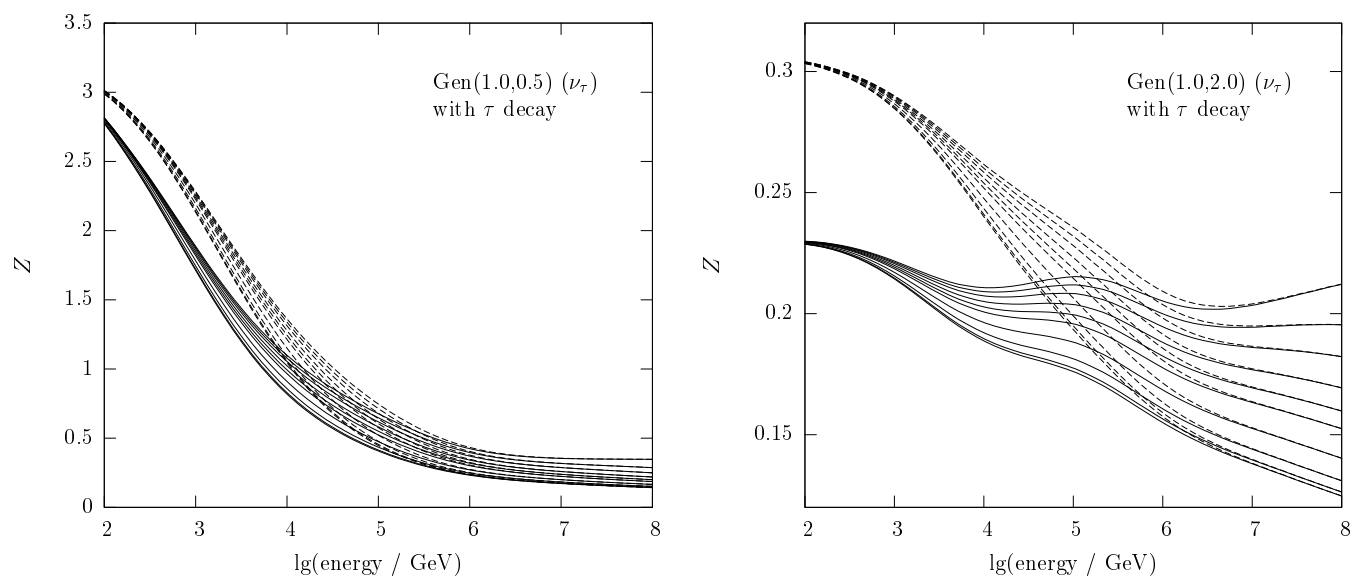

Figure 5.28: Left: $Z$ factor of tau neutrinos (solid) and tau antineutrinos (dashed lines) after crossing the Earth at various nadir angles. Gen $(1.0,0.5)$ is assumed as the initial neutrino spectrum. The nadir angle is increased in steps of $10^{\circ}$ from $0^{\circ}$ (bottom) to $90^{\circ}$ (top lines). Right: The same as the left panel, but for Gen(1.0,2.0) as the initial spectrum.

energies below $\sim 10^{6} \mathrm{GeV}$. An explanation of this feature has already been given above.

Thirdly, comparing the right panels of Figs. 5.24 5.27 to Fig. (2) of [153], we see that the curves are basically consistent with each other, as should be expected. However, the different cross sections used imply some small differences. Furthermore, comparing the left and right panels of the figures, we reach the conclusion that ignoring the tauon decay may have drastic consequences and may alter the $Z$ factor values by a factor of up to 2 . This underlines the importance of taking tau neutrino propagation into account, even if one is concerned with muon (or, for that matter, electron) neutrinos only.

Whereas the respective plots for electron neutrino $Z$ factors would almost look the same as the ones for muon neutrinos, the tau neutrino plots can be significantly different. As an example, the cases of Gen(1.0,0.5) and Gen $(1.0,2.0)$ are shown in Fig. 5.28. Further plots can be obtained by means of TEMPEST (cf. Sect. A.1). 

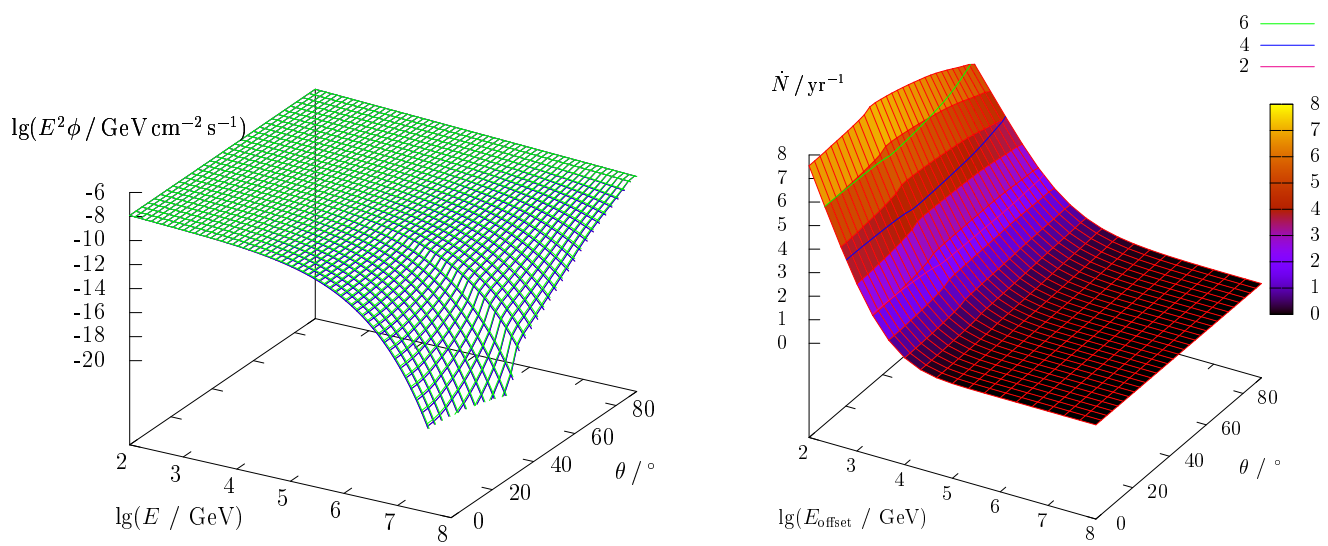

Figure 5.29: Left: Neutrino flux for the supernova remnant RX J1713.7-3946 as a function of the neutrino energy and the nadir angle under which the neutrinos cross the Earth. [56] As in the following plots, the flux is an estimate. Right: Corresponding muon event rate for the generic detector described in Sect. 4.2.

\subsection{Galactic sources}

Several hours prior to the first confirmed optical observation of the supernova SN 1987A in the Large Magellanic Cloud, which blazed off on 23 February 1987, the Kamiokande, IMB and Baksan detectors at 7:35 UT observed 12, 8 and 5 neutrinos, respectively. For Kamiokande and IMB, these numbers presumably include one background neutrino. [104, 47, 11, 168] So far, SN 1987A has remained the only extrasolar neutrino source seen on Earth. Alas, the energy range of its neutrinos was below that considered in this work.

Not all is lost, though. As the supernova remnant (i.e. the debris cast off at the supernova explosion) protrudes into the interstellar medium, shocks arise, at which (in addition to electrons) protons might be accelerated. Indeed, the CANGAROO detector has provided evidence that the supernova remnant RX J1713.7-3946 emits $\gamma$-radiation arising from $\pi^{0}$ decay. [73] Hence, this supernova remnant constitutes an excellent candidate for neutrino observations, as can be seen from Fig. 5.29. [14, 56]

In addition, heavy nuclei (such as iron nuclei) may be accelerated in the magnetosphere of a pulsar and subsequently photo-disintegrate due to interactions with the soft photons in the outer gap. If the resulting neutrons decay inside the nebula around the pulsar, the protons from the decay are confined by its magnetic field. These may then undergo $p p$ interactions, so 

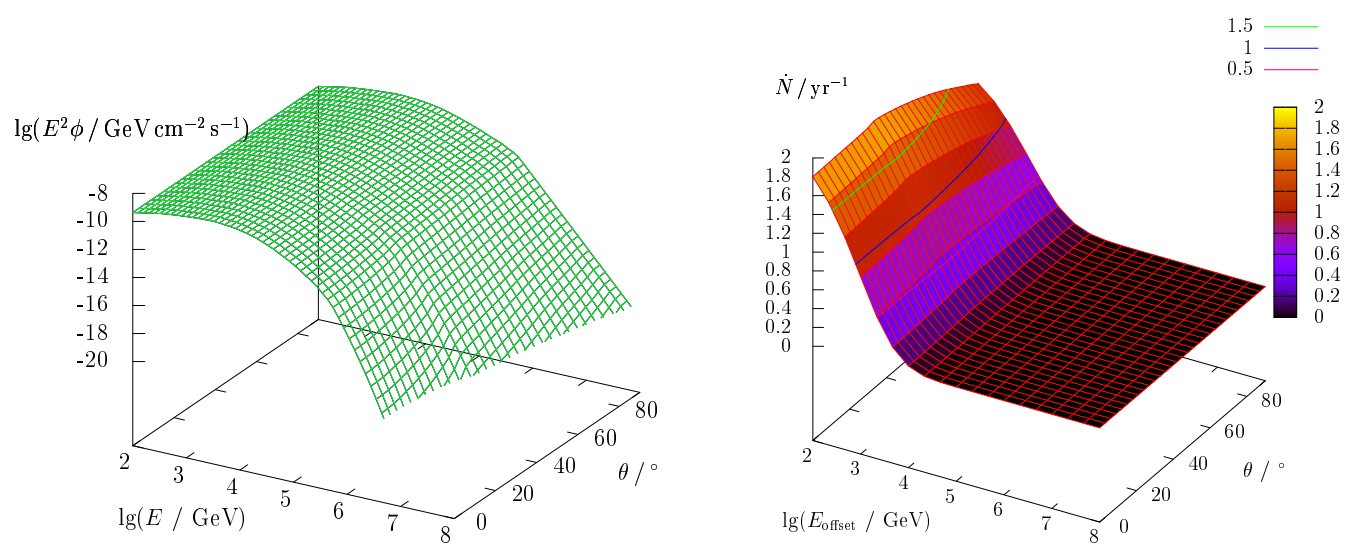

Figure 5.30: Left: Neutrino flux for the Crab nebula, assuming model I of [37]. Right: Corresponding muon event rate.

that neutrinos are created. [37] The corresponding flux for the Crab nebula is shown in Fig. 5.30. Furthermore, it was pointed out in [152] that neutrinos might result from $p p$ interactions in the pulsar wind as well (see Fig. 5.31).

The accretion of matter from a giant star onto a neutron star or black hole in an X-ray binary may lead to the formation of twin jets. If so, one speaks of a galactic microquasar. [149] Similarly to the case of AGN jets discussed below, shocks may form in microquasar jets, and protons might be accelerated and interact with the synchroton radiation of accelerated electrons to give neutrinos. [131] As an example, the respective flux for the persistent source SS433 is given in Fig. 5.32. [131, 63]

Magnetars (i.e. pulsars with an extremely high magnetic field strength of $B \sim 10^{11} \mathrm{TeV}$ ) may emit giant flares, which are thought to be caused by a reconfiguration of their magnetic field. [185, 109] During the third such burst, which was emitted by SGR 1806-20 and detected on 27 December 2004, an overall energy of $\sim 2 \times 10^{46} \mathrm{erg}$ was released within 0.2 s. [106, 159] If no corresponding neutrino flux was measured by AMANDA, constraints may be put on the flare model (cf. Fig. 5.33). [110]

The HEGRA telescope detected an extended TeV source of $\gamma$-rays inside Cygnus-OB2 [3], a young globular cluster at a distance of $1.7 \mathrm{kpc}$ [119], and there is evidence that this flux arises from neutrons decaying on their way to Earth. If so, there should be a corresponding neutrino flux, as illustrated in Fig. 5.34. [18]

An emission of $\gamma$-radiation from the Galactic Center has been observed 

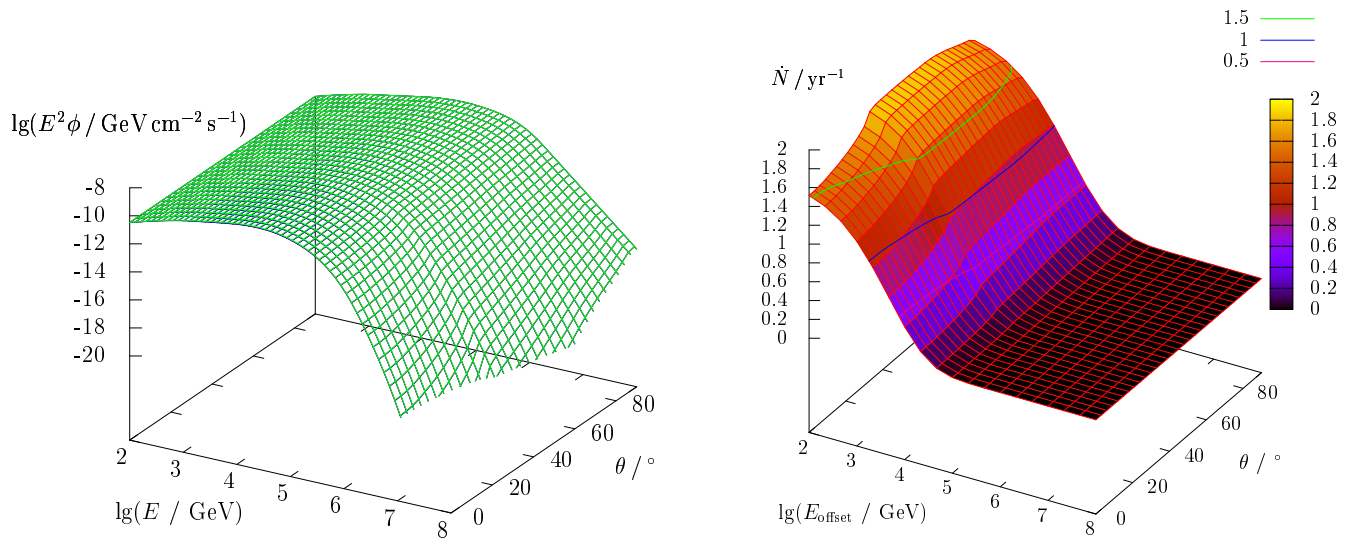

Figure 5.31: Left: Neutrino flux from a pulsar wind with a Lorentz factor of $\Gamma=10^{6}$ for a pulsar aged 1 year with a period of $1 \mathrm{~ms}$, and a magnetic field of $10^{8} \mathrm{~T}$ at a distance of $10 \mathrm{kpc}$ from Earth. [152] Right: Corresponding muon event rate.
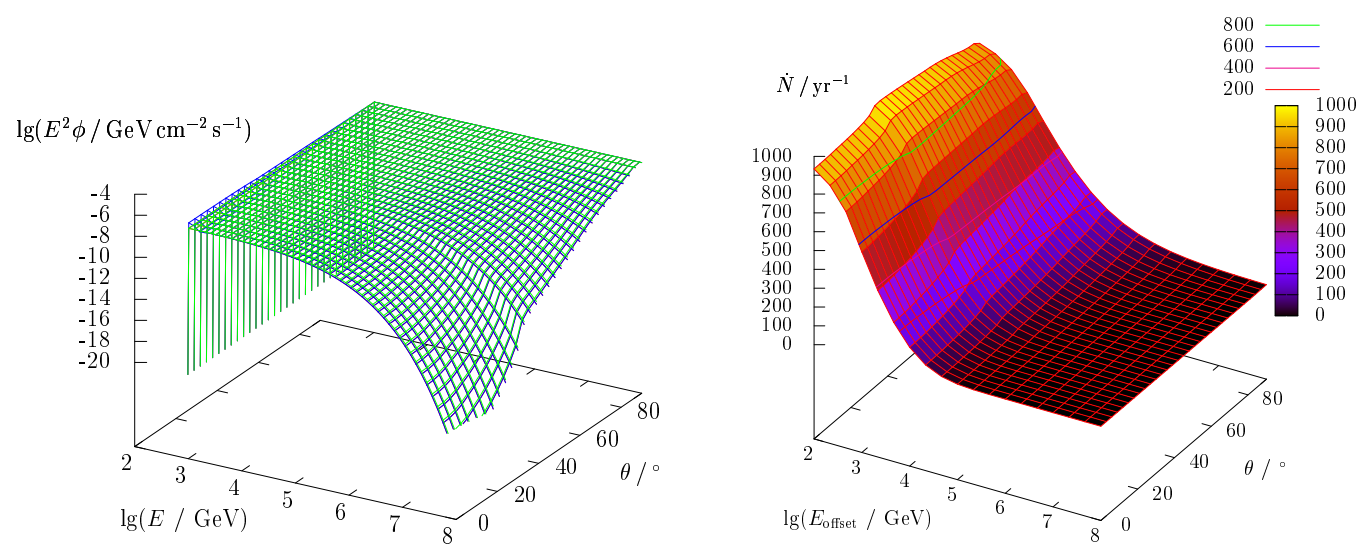

Figure 5.32: Left: Neutrino flux from the jets of the galactic microquasar SS433. [131, 63]. Right: Corresponding muon event rate. 

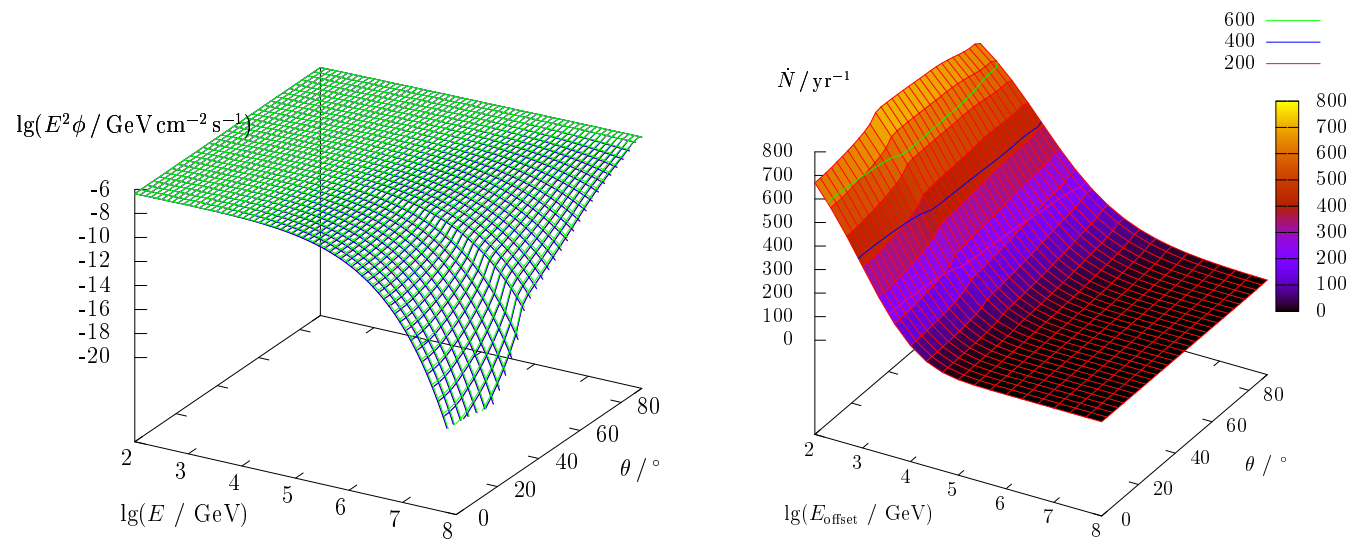

Figure 5.33: Left: Neutrino fluence divided by 1 yr for the giant flare of the magnetar SGR 1806-20 on 27 December 2004. [110] Right: Corresponding number of muon events divided by $1 \mathrm{yr}$.
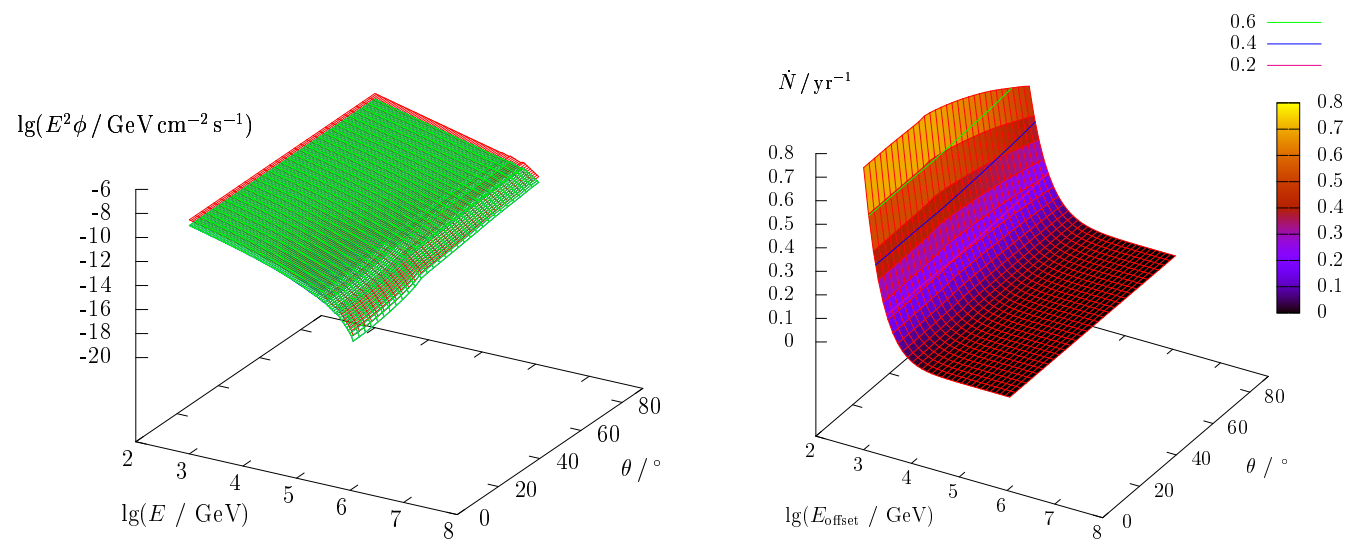

Figure 5.34: Left: Neutrino flux for the globular cluster Cyg OB2, if its $\mathrm{TeV}$ $\gamma$-radiation is due to neutrons decaying on their flight to Earth. [18] Right: Corresponding muon event rate. 

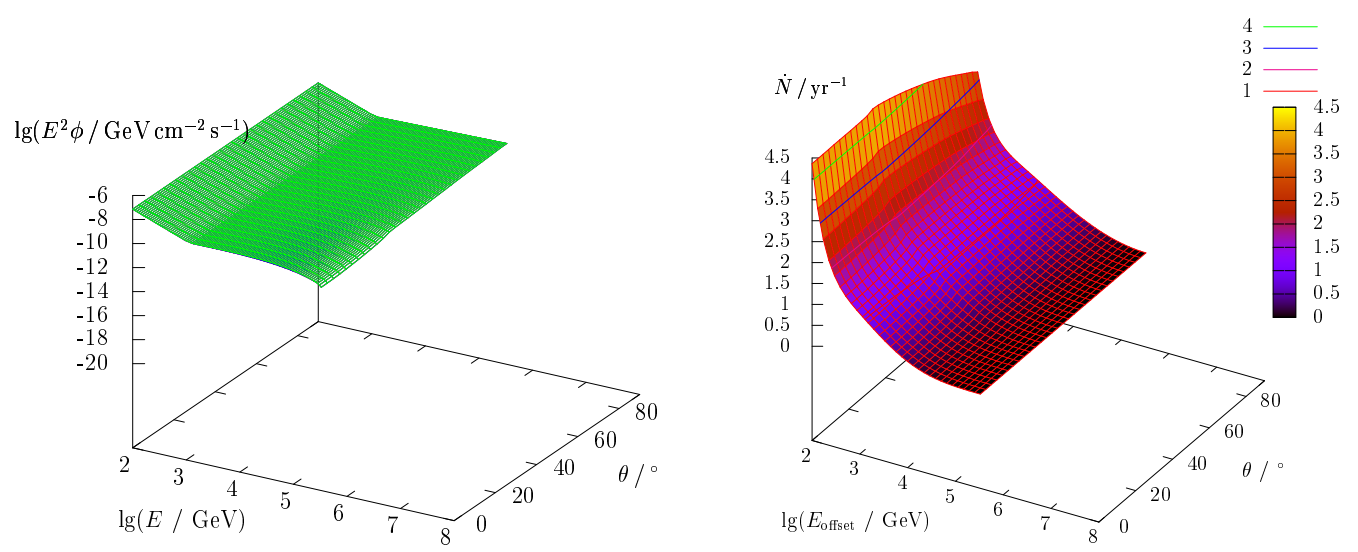

Figure 5.35: Left: Neutrino flux from the Galactic Center, as inferred from HESS and extreme high energy cosmic ray observations. [57] Right: Corresponding muon event rate.

(among others) both by EGRET and HESS [141, 4], which seems to be produced by the deacay of neutral pions originating from $p p$ interactions. Unfortunately, it is still unclear whether the measured spectra are due to more than one source and whether $\gamma \gamma$ attenuation plays a role. [57] In Fig. 55.35, a neutrino flux estimate based on the HESS and extreme high-energy cosmic ray observations is shown.

Cosmic ray protons propagating through the Galaxy can undergo $p p$ interactions with the interstellar matter [26], which leads to a neutrino flux from the Galactic plane, as given by Fig. 5.36 .

Finally, the cosmic ray density depends on the location in the Galaxy; for example, the density in the Galactic Center exceeds the local one by a factor of ten. [50] Hence the corresponding neutrino flux depends on the direction, as illustrated by Figs. 5.37 and 5.38, which show neutrino fluxes for the Galactic Center and the galactic coordinates $b=0, l=45^{\circ}$, respectively.

\subsection{Extragalactic sources}

One of the key candidates for extragalactic neutrino sources are gamma-ray bursts (GRBs), which constitute short (30 ms - $100 \mathrm{~s}$ ) bursts of $\gamma$-radiation with an energy exceeding $0.1 \mathrm{MeV}$. [79] They were detected by the Vela satellites [117], the main task of which was to monitor the ban on atmospheric 

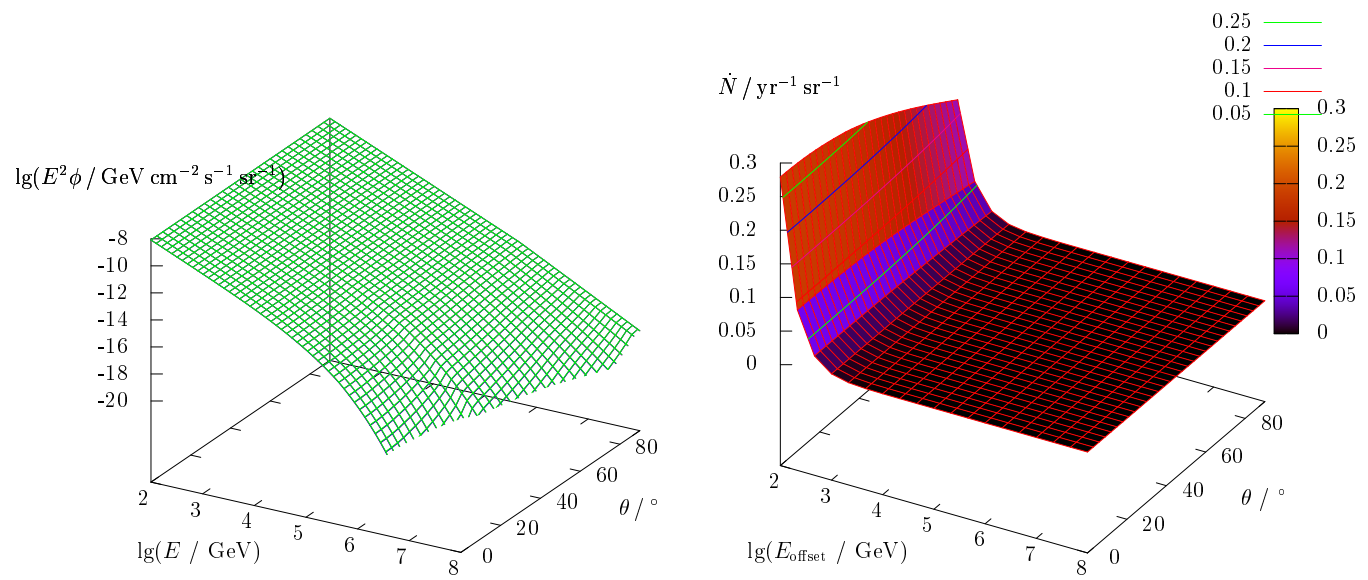

Figure 5.36: Left: Neutrino flux from the Galactic plane according to [26]. Right: Corresponding muon event rate.
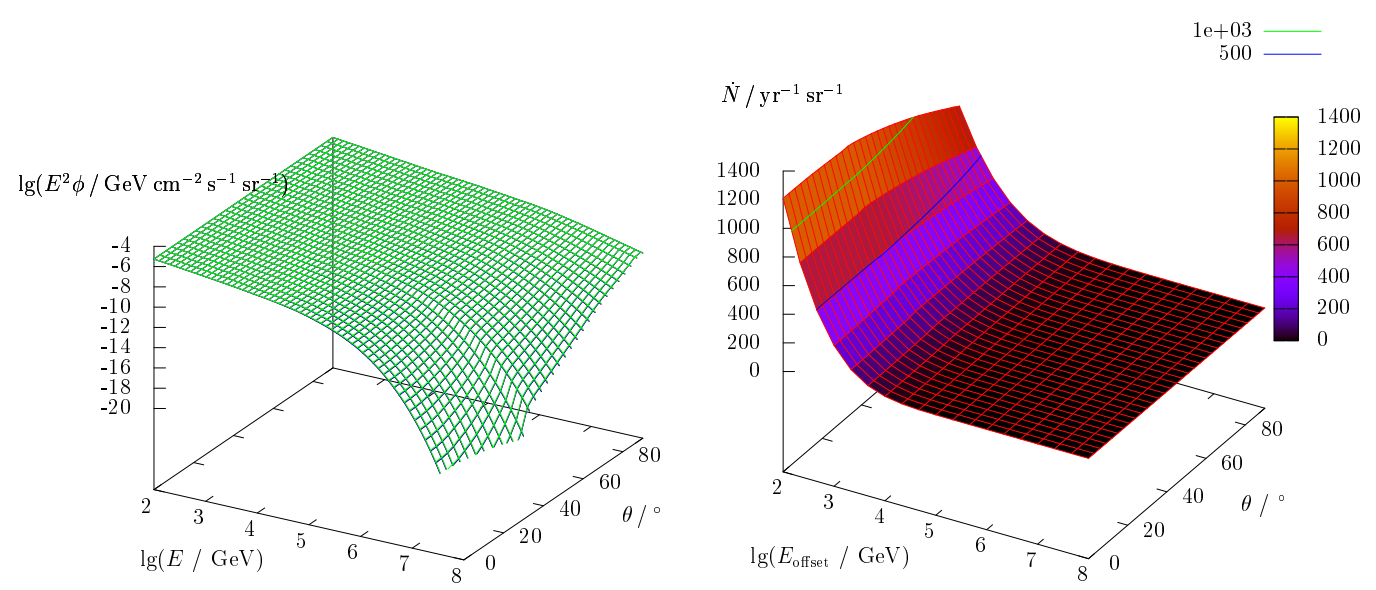

Figure 5.37: Left: Neutrino flux from the Galactic Center, as given by [50]. Right: Corresponding muon event rate. 

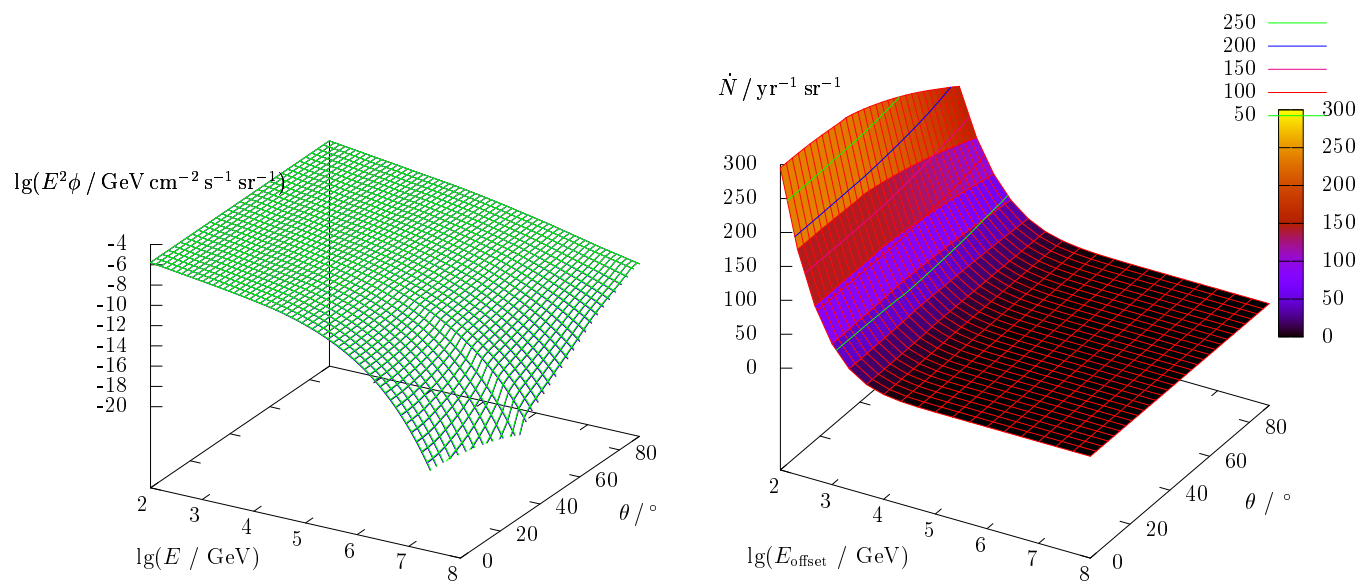

Figure 5.38: Left: Galactic neutrino flux from the direction $b=0^{\circ}, l=45^{\circ}$, as given by [50]. Right: Corresponding muon event rate.

tests of nuclear bombs.

For more than two decades, the nature of GRBs remained a mystery. Observations by means of BATSE yielded a spatial distribution inconsistent with galactic sources. [142] The real breakthrough, however, came in 1997, when BeppoSAX was able to measure the the position of GRB970508 with sufficient precision, so that an X-ray and optical afterglow could be found. [55, 65] As the latter could be associated with a galaxy of known redshift, the distance of GRB970508 was established to be greater than $z \geqslant 0.835$, confirming an extragalactic origin. [145]

Thus it became clear that during a GRB an energy of $10^{51}-10^{54} \mathrm{erg} / \mathrm{s}$ (i.e. $0.005-0.5 M_{\odot} c^{2}$ ) is released. Its origin might either be a hypernova or a collision of a neutron star with another neutron star or a black hole. [143] Irrespective of the precise details, a highly relativistic fireball consisting of $e^{+} e^{-}$and photons is formed, which expands with a Lorentz factor of $\Gamma \sim$ 300. [52, 189]

Electrons may be accelerated at collision-less shocks inside the fireball and at the border between the fireball and the surrounding medium. Whereas the former is thought to give rise to the observed $\gamma$-radiation, the latter may explain the afterglow. [189]

Similarly, protons can be accelerated inside the fireball. If these interact with the $\gamma$-radiation, pions may be produced via a $\Delta$-resonance, and hence 
neutrinos are created according to Eqs. 5.1 and 5.3:

$$
p+\gamma \longrightarrow \Delta \longrightarrow n+\pi \longrightarrow n+\mu+\nu_{\mu} \longrightarrow n+e+\nu_{e}+\nu_{\mu}+\nu_{\mu}
$$

In the observer frame, the proton energy $E_{p}$ must fulfill the condition

$$
E_{p} \geqslant \frac{\left(m_{\Delta}^{2}-m_{p}^{2}\right) \Gamma^{2}}{4 E_{\gamma}},
$$

and as the $\Delta$ resonance has a mass of $m_{\Delta}=1232 \mathrm{MeV}$ [70], this translates into

$$
E_{p} \geqslant 1.4 \times 10^{16}\left(\frac{\Gamma}{300}\right)^{2}\left(\frac{E_{\gamma}}{1 \mathrm{MeV}}\right) \mathrm{eV} .
$$

As about $5 \%$ of the initial proton energy is transferred to a neutrino, we see that one may expect GRB neutrinos to have an energy of $E_{\nu} \geqslant 100 \mathrm{TeV}$. [189] Their spectrum basically follows that of the observed $\gamma$-radiation, albeit shifted to higher energies. At energies above $\sim 10^{8} \mathrm{GeV}$, however, the synchrotron losses of pions and muons prior to their decay have to be taken into account. [15]

Alternatively, provided that the surrounding medium is sufficiently dense, the accelerated protons might interact with other nucleons. [160] The flux and event rate estimated for such a GRB model are shown in Fig. 5.39.

Individual GRBs with a sufficiently large fluence will presumably be observable in next-generation neutrino telescopes. GRB941017 is considered as an example in [15]; its estimated neutrino fluence and the corresponding number of events are given in Fig. 5.40.

An analysis similar to that performed in Sect. 5.5 can be used to obtain upper flux limits for GRB-like sources. The results corresponding to the MPR and the WB flux bound are given in [138] and [190], and they are shown in Figs. 5.41 and 5.42, respectively.

Note that in the calculation of the curve corresponding to the MPR flux bound, no assumptions concerning the proton spectrum inside the fireball is made. A more realistic approach may be found in [138] as well.

In addition, protons may interact with optical and UV photons of the GRB afterglow. Here, the $\Delta$ resonance condition (Eq. 5.8) implies a proton energy of $\approx 10^{20} \mathrm{eV}$ and thus neutrino energies of the order of $10^{18} \mathrm{eV}$. [191] A typical resulting neutrino spectrum and the corresponding event rate are shown in Fig. 5.43 . 

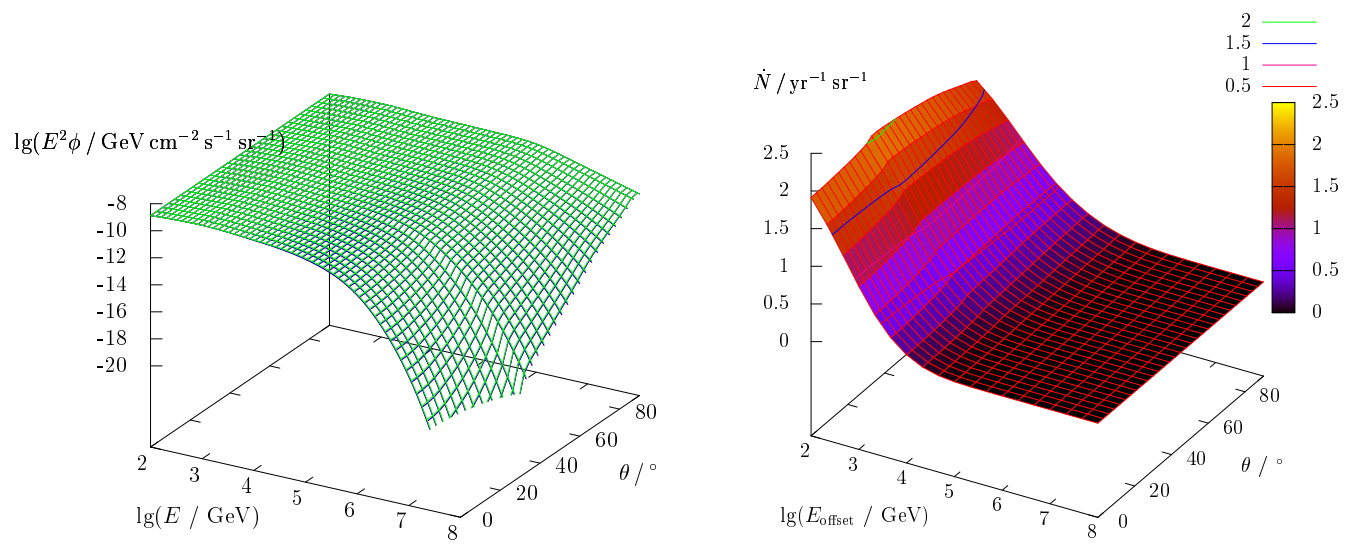

Figure 5.39: Left: Neutrino flux due to proton-nucleon interactions in gammaray bursts, as estimated by curve $\mathrm{pN}(2)$ of [160]. As in the preceding section, the fluxes in this and the following plots should be understood as estimates. Right: Corresponding muon event rate.
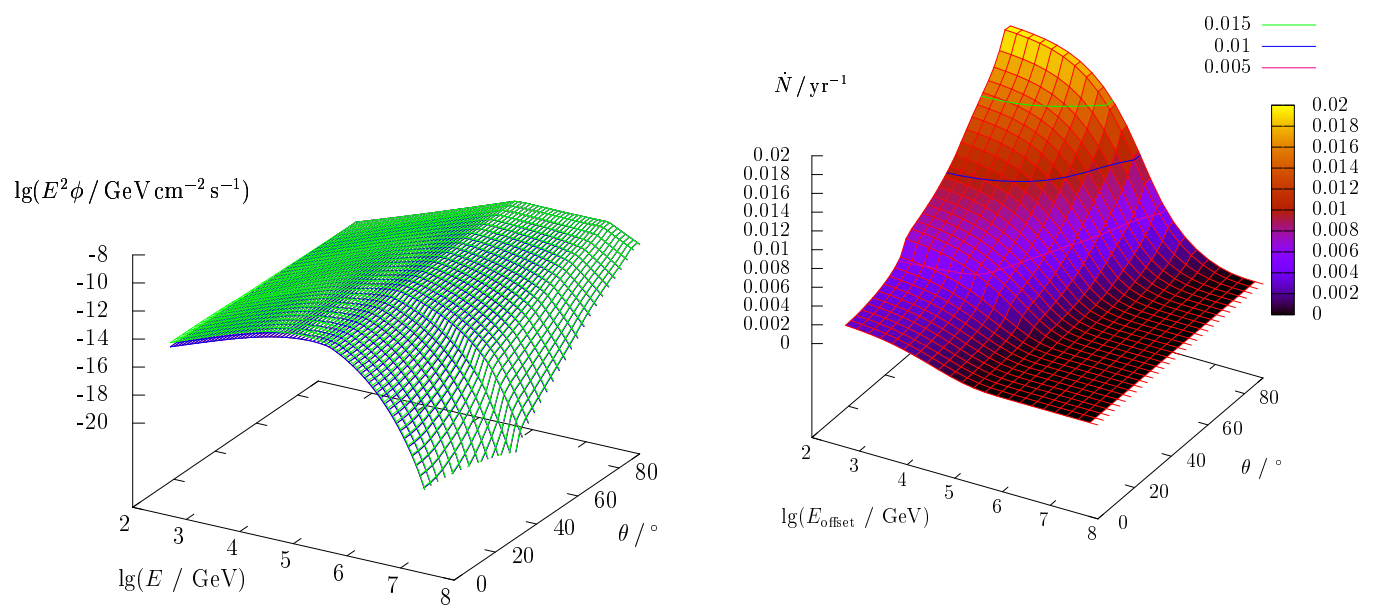

Figure 5.40: Left: Neutrino fluence of GRB941017 divided by 1 yr. [15] Right: Corresponding number of muon events divided by $1 \mathrm{yr}$. 

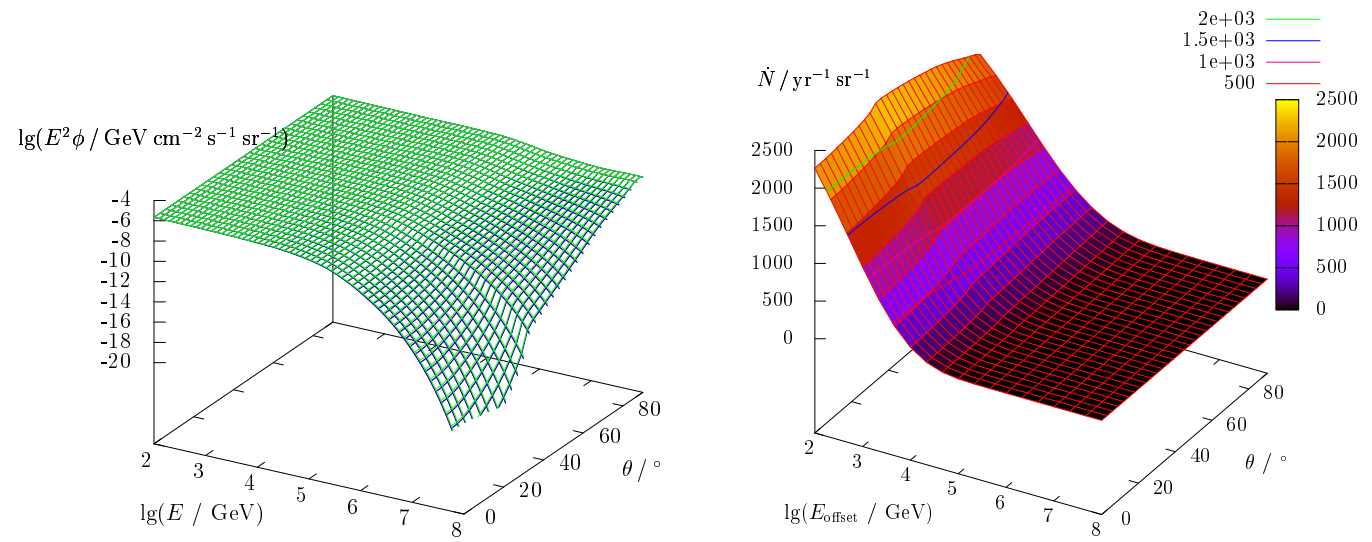

Figure 5.41: Left: Upper neutrino flux bound for GRB-like sources obtained analogically to the MPR flux bound. [138] Right: Corresponding muon event rate.
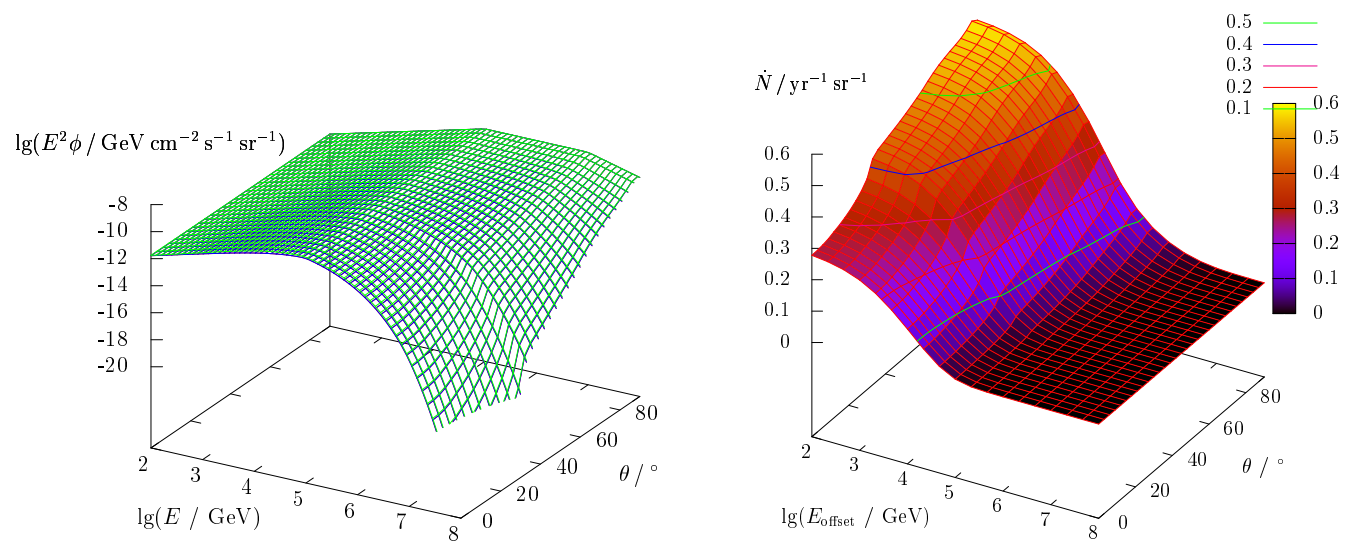

Figure 5.42: Left: Upper neutrino flux bound for GRB-like sources obtained analogically to the WB flux bound. [190] Right: Corresponding muon event rate. 

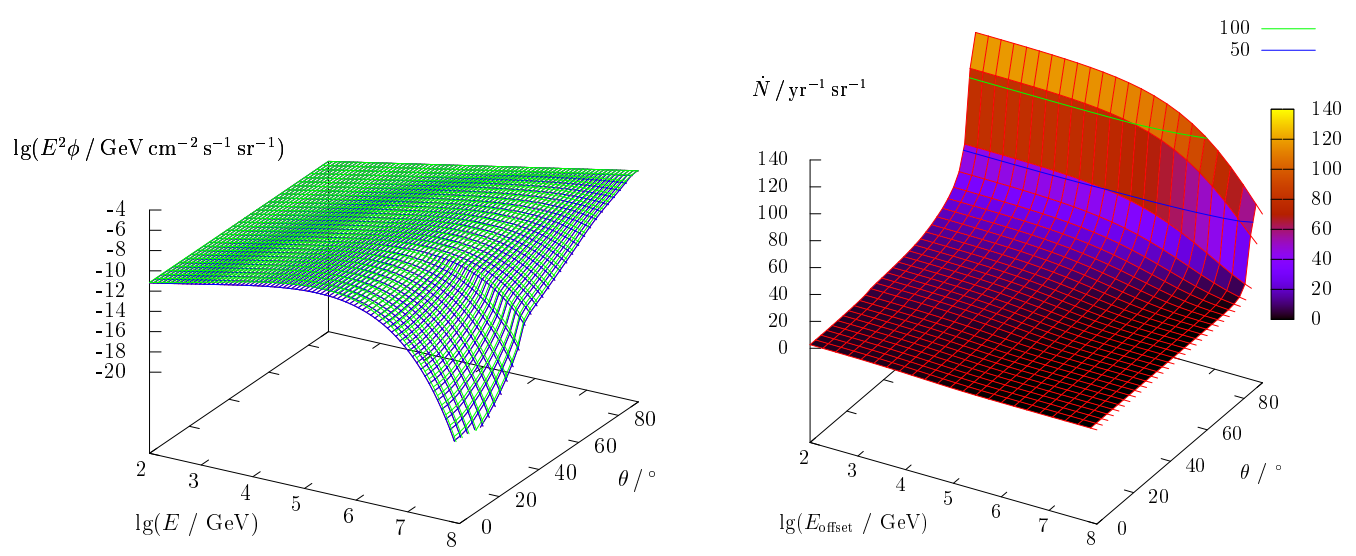

Figure 5.43: Left: Neutrino flux arising from proton interactions with GRB afterglow photons. [191] Right: Corresponding muon event rate.

If the ratio of luminosity and mass injection rate is sufficiently large, protons and neutrons may decouple in the fireball during the proton acceleration, so that a speed difference between the two ensues. In this case, proton-neutron interactions give rise to a flux of electron and muon neutrinos with energies of about 5-10 GeV. Corresponding event rates of $\sim 10$ events per year might be possible in next-generation neutrino detectors. [29] Due to their low energy, a propagation through the Earth doesn't affect the spectrum of these neutrinos.

In case the GRB progenitor is a core collapse of a massive star, the fireball has to work its way through the stellar envelope. This causes a shock inside the envelope, at which protons may be accelerated. These protons can subsequently interact with thermal X-ray photons and thus produce electron and muon neutrinos with energies $\gtrsim 5 \mathrm{TeV}$. An event rate of $0.1-10$ events per burst might be possible in $\mathrm{km}^{3}$ detectors. [144] Again, due to the low energies involved, a propagation through the Earth has no implications for the neutrino spectrum.

While GRBs are the most energetic explosions in the cosmos, active galactic nuclei (AGNs) constitute the most energetic steady sources. They may have bolometric luminosities exceeding $10^{42} \mathrm{erg} / \mathrm{s}$, and they display jets the length of which may reach several hundred kpc. [192]

This suggests that AGNs are powered by matter accreting on a (possibly rotating) black hole with a mass of up to several hundred million solar masses. [114] Indeed, measurements of the stellar velocities in the central 

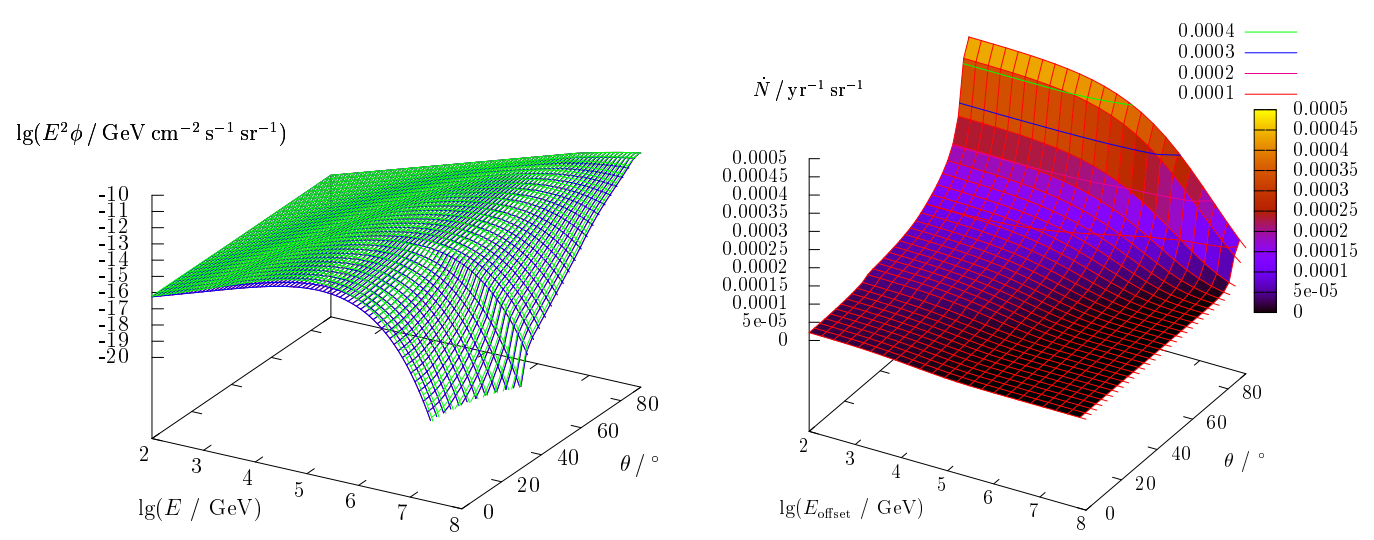

Figure 5.44: Left: Neutrino flux from $p \gamma$ interactions in the jets of nominal AGNs. [137] Right: Corresponding muon event rate.

region of our own Galaxy have revealed the presence of a black hole with a mass of $(3.7 \pm 1.5) \times 10^{6} M_{\odot}$ [178], and near-infrared flares from Sgr A*, which are thought to arise from accreting matter, have provided evidence for a rotation of this black hole. [88]

In the AGN jets, electrons are accelerated at shocks, which as usual gives rise to synchrotron photons. In addition, the accretion disk emits thermal radiation. [166] Hence, if protons are accelerated in the jet, these may interact with this radiation, so that neutrinos are produced. [136, 137]. A typical neutrino spectrum to be expected for this process is given in Fig. 5.44.

Due to the low matter density, $p p$ interactions in an AGN jet are rare. However, if accelerated protons diffuse back to the accretion disk, they may undergo $p p$ interactions in the disk: [155]

$$
p+p \longrightarrow p+N+\pi
$$

Alternatively, protons might be accelerated at shocks in the accretion disk itself and undergo proton-photon interactions with the thermal disk radiation. [182] The resulting neutrino spectrum for the case of proton-proton and proton-photon interactions in the blazar 3C273 [155, 127] and the corresponding event rate are shown in Figs. 5.45 and 5.46, respectively.

About $90 \%$ of the AGNs are radio-quiet, as they lack a prominent jet. [114] This may be explained by assuming that in these AGNs, the outflow doesn't reach the escape velocity, so that "the jet is choked". If so, as the outflow is intermittent, blobs will collide in the central region, and hence there will 

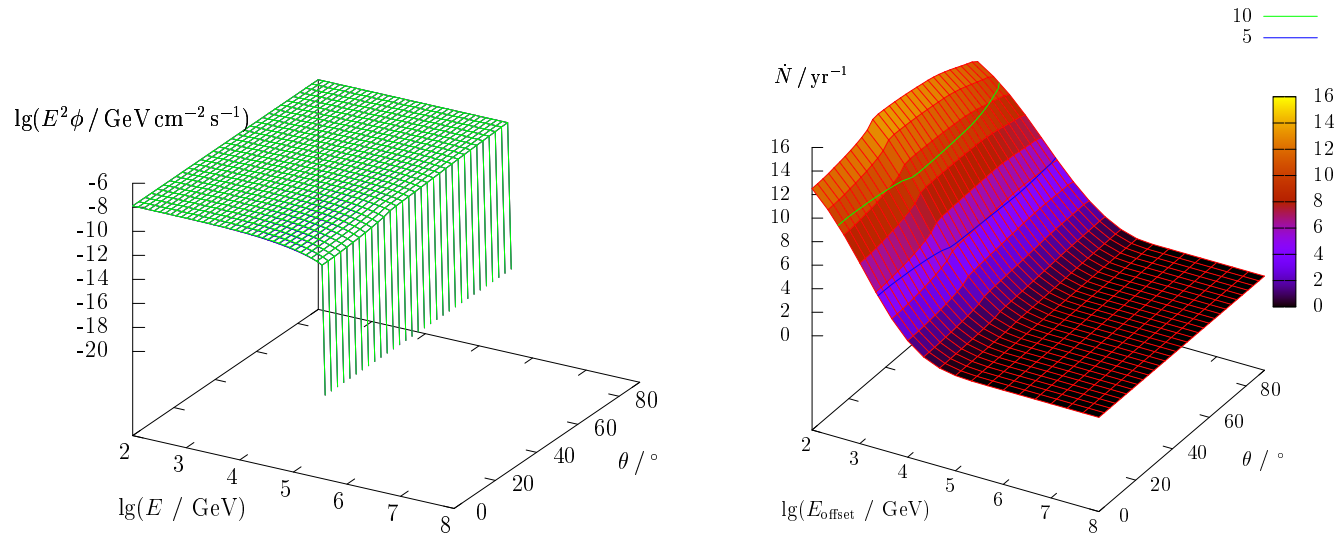

Figure 5.45: Left: Neutrino spectrum of the blazar 3C273 under the assumption that accelerated protons diffuse from the jet to the accretion disk and undergo $p p$ interactions. [155, 127] Right: Corresponding muon event rate.
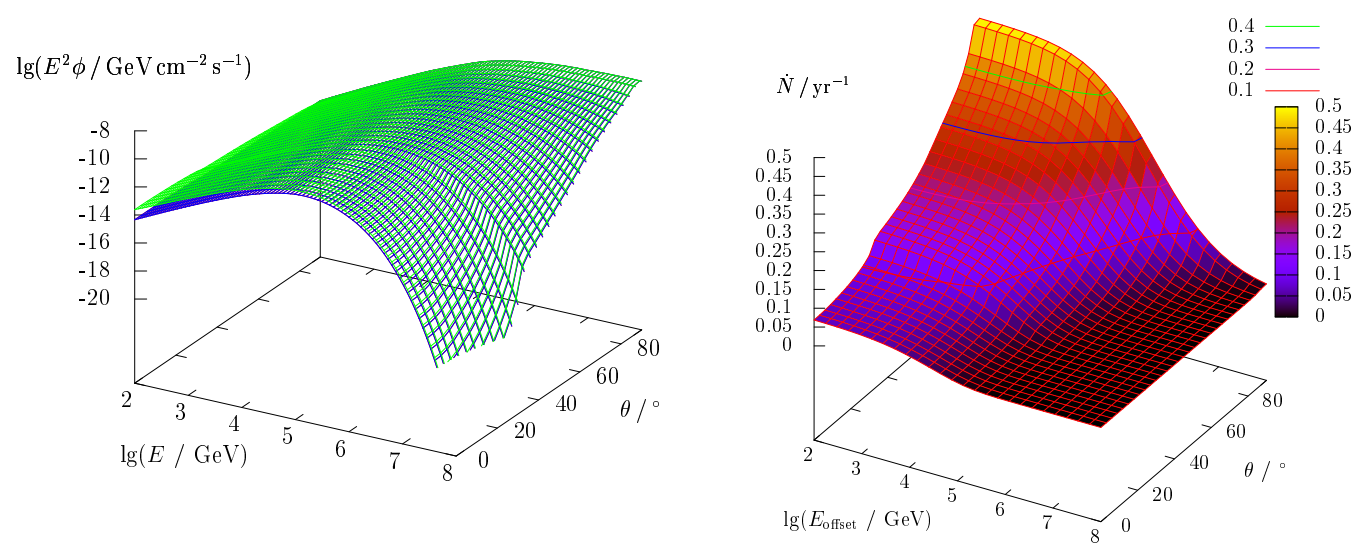

Figure 5.46: Left: Neutrino spectrum of the blazar 3C273 under the assumption that protons are accelerated in the accretion disk and undergo $p \gamma$ interactions with the thermal radiation. [182] Right: Corresponding muon event rate. 

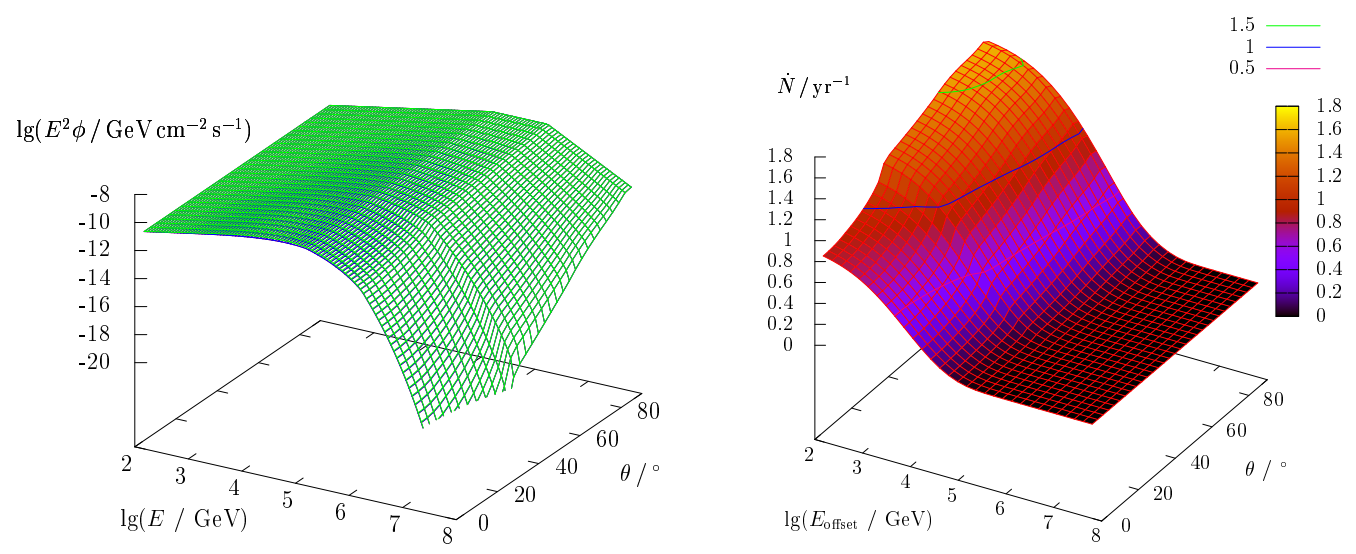

Figure 5.47: Left: Neutrino flux from a nominal radio-quiet AGN at a distance of $20 \mathrm{Mpc}$. [16] Right: Corresponding muon event rate.

be shocks at which protons can be accelerated. These protons may subsequently interact with both disk photons and (cool) disk protons, giving rise to a neutrino flux, as shown for a nominal radio-quiet AGN in Fig. 5.47. [16]

In [35], the neutrino flux (under the assumption of $p \gamma$ interactions) is integrated over all FR-II radio galaxies and blazars. The resulting flux is covered by Fig. 5.48 .

Possibly, the AMANDA telescope has already detected two neutrinos from the TeV blazar 1ES 1959+650. [170] As illustrated in [96], this would be consistent with a flux estimate based on the gamma-ray luminosity of this blazar (cf. Fig. 5.49).

Neutrinos may also be copiously produced during the formation of a massive black hole in a galactic center. Indeed, assuming that the black hole is created from a collapsing stellar cluster, one has to expect both a neutron star cluster and an envelope due to disrupted stars. In addition, the stellar collisions lead to a repeated creation of fireballs, which in turn give rise to the formation of rarefied cavities, in which protons can be accelerated.

These protons can subsequently interact with envelope protons via $p p$ interactions, thus producing neutrinos. If the neutron star cluster and the envelope are formed simultaneously, this neutrino flux should persist for $\sim 10$ years. [38] If, on the other hand, the neutron star cluster is formed later than the envelope (inside a supermassive star), the resulting neutrino flux is higher, but of shorter duration $(\sim 0.1-1$ year $)$. [39] These two cases are covered by Figs. 5.50 and 5.51 , respectively. 

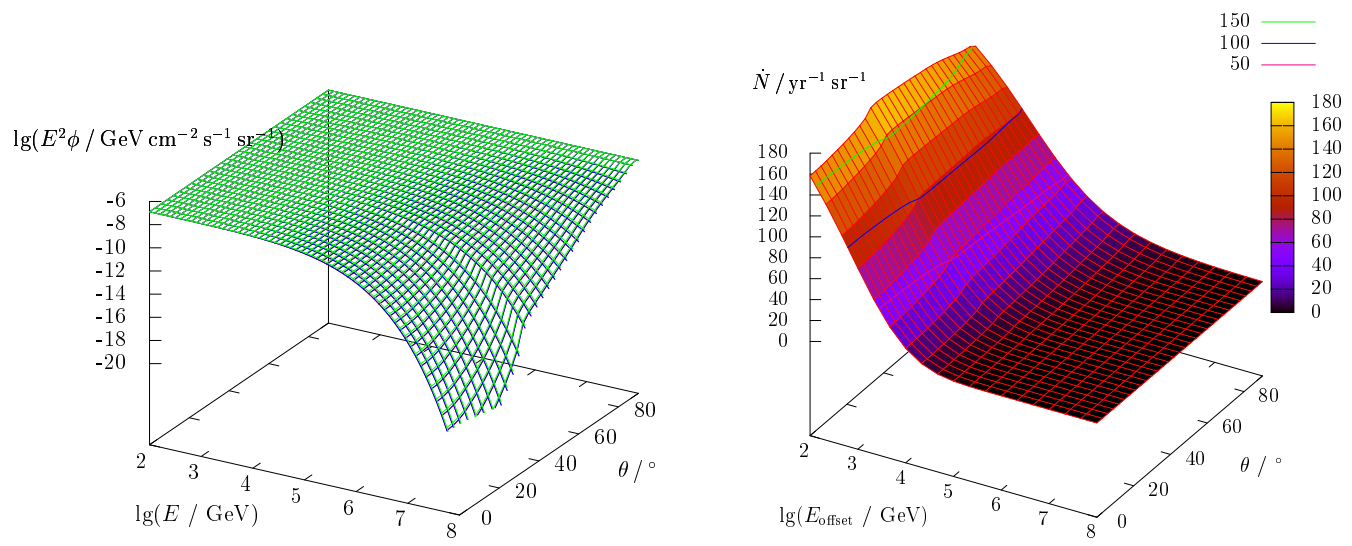

Figure 5.48: Left: Neutrino flux of all FR-II radio galaxies and blazars. [35] Right: Corresponding muon event rate.
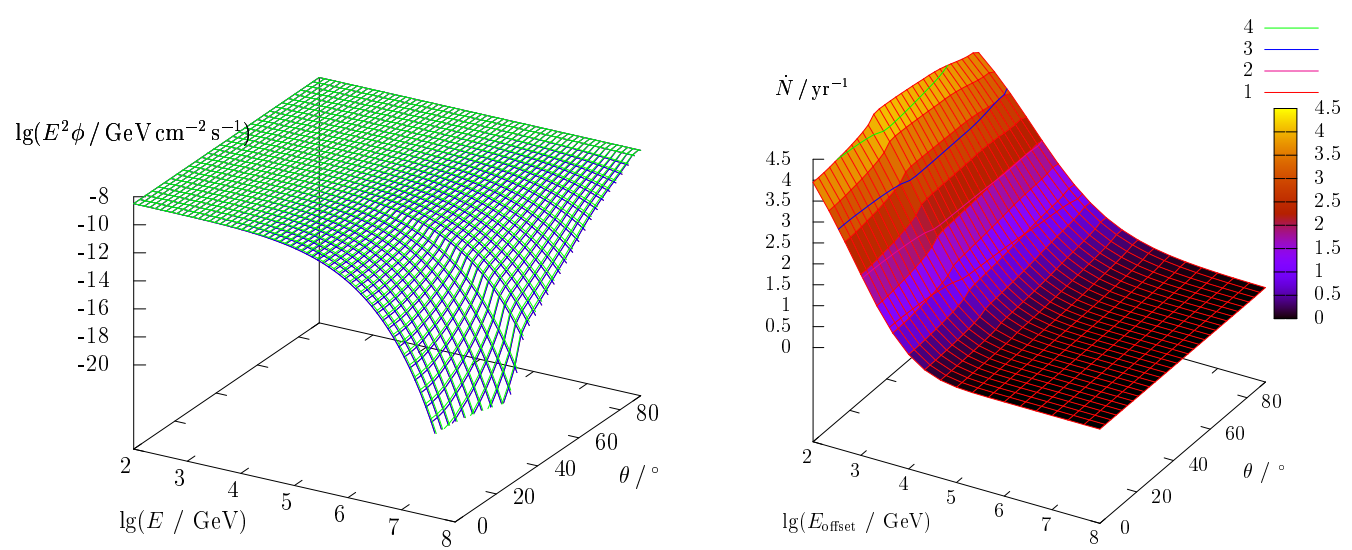

Figure 5.49: Left: Neutrino flux estimated for the TeV blazar 1ES 1959+650. [96] Right: Corresponding muon event rate. 

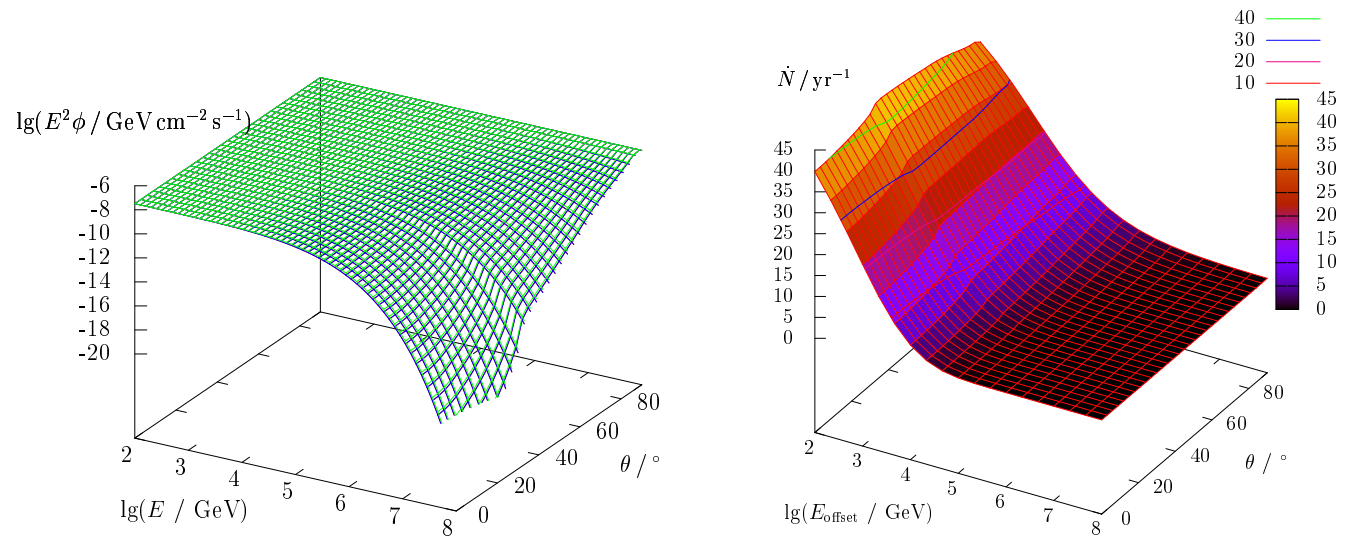

Figure 5.50: Left: Neutrino flux from the formation of a massive black hole, assuming that the precursor neutron star cluster is formed at the same time as the envelope from stellar disruptions. A distance of $1 \mathrm{Gpc}$ is assumed. [38] Right: Corresponding muon event rate.
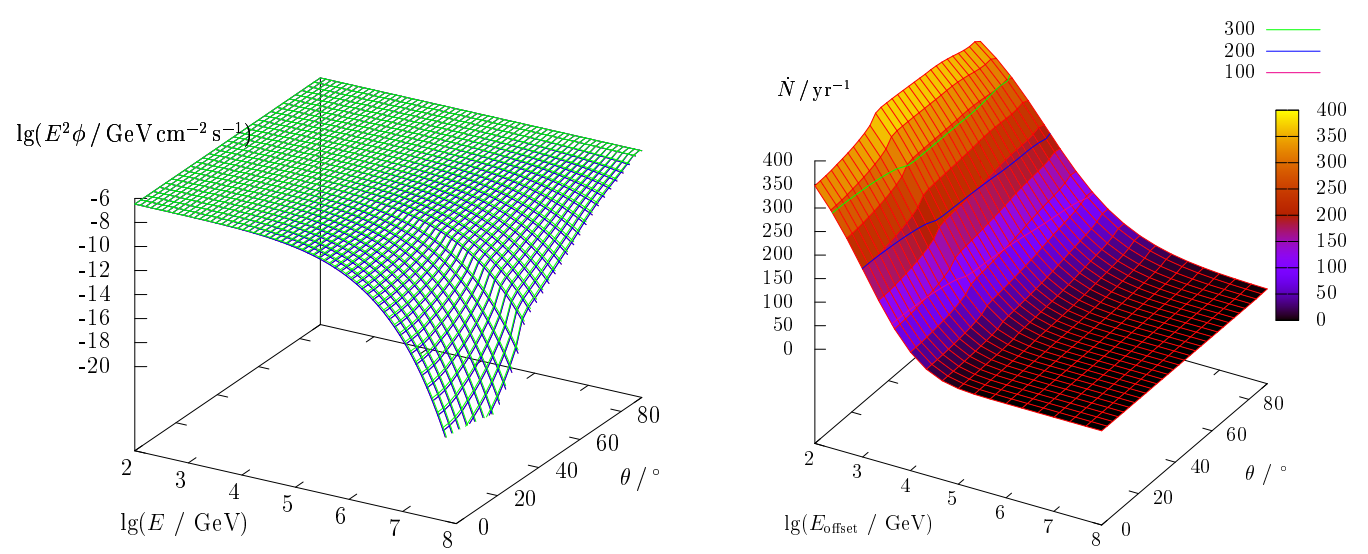

Figure 5.51: Left: Neutrino flux from the formation of a massive black hole, assuming that the precursor neutron star cluster is formed after the envelope from stellar disruptions. A distance of $1 \mathrm{Gpc}$ is assumed. [39] Right: Corresponding muon event rate. 

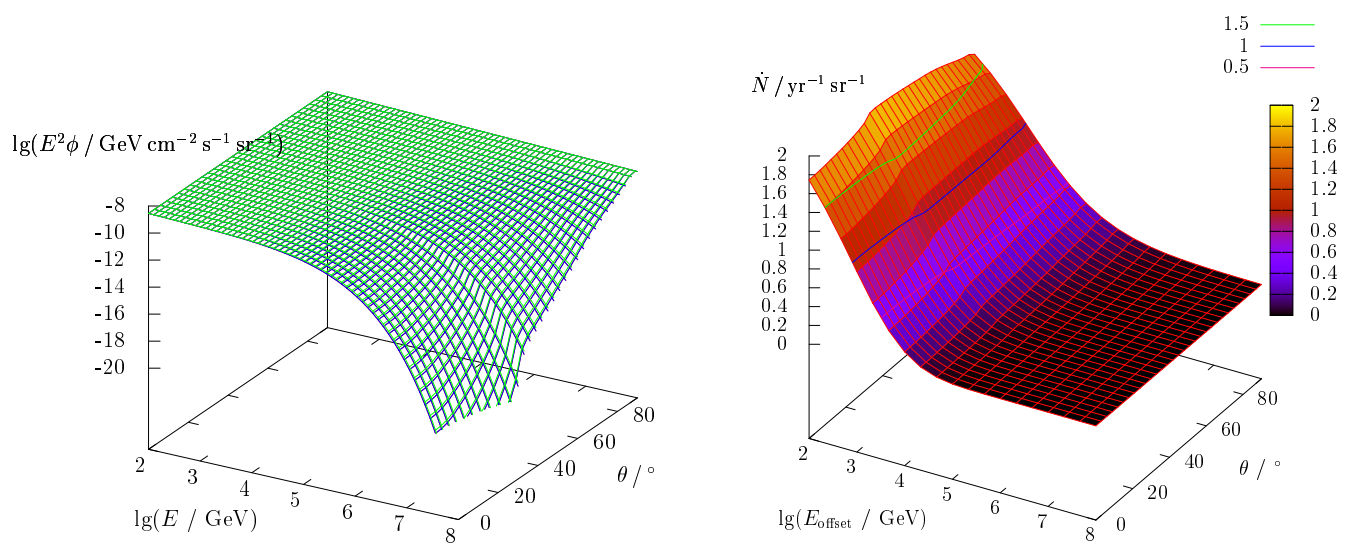

Figure 5.52: Left: Neutrino flux from galaxy clusters. [54] Right: Corresponding muon event rate.

Cosmic-ray protons may suffer $p \gamma$ interactions with the cosmic microwave background photons. The energy of the latter is of the order of $250 \mu \mathrm{eV}$, and the threshold energy for this process (known as the GZK cutoff) is of the order of $10^{11} \mathrm{GeV}$. [94] This seems to be a guaranteed neutrino source.

However, if a significant fraction of the ultrahigh-energy cosmic rays consists of heavy nuclei rather than protons, the cosmic ray particles undergo photo-disintegration by interacting with the cosmic infrared background. This implies that the neutrino flux expected from cosmic ray propagation is shifted to lower energies (see [105, 28] for respective spectra).

Concerning a galaxy cluster, high energy cosmic rays may be produced both in its normal and active galaxies by the sources described so far, and at accretion shocks due to its collapse and virialization. As the cosmic rays may be confined in the cluster, the likelihood of $p p$ interactions can be enlarged significantly, so that the neutrino flux given in Fig. 5.52 [54] might ensue.

\subsection{Flux limits}

Even though no high energy neutrino source has been observed yet, it is possible to make some reliable assertions concerning the maximum neutrino flux to be expected from such sources. The idea behind deriving such flux limits is illustrated in Fig. 5.53: In a hadronic particle accelerator, protons are accelerated to high energies and subsequently interact with ambient photons 


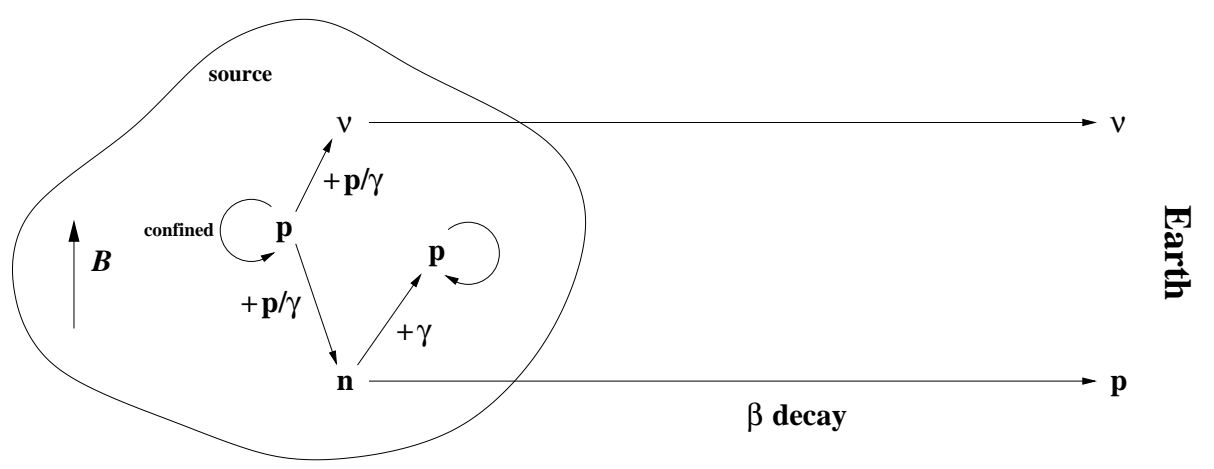

Figure 5.53: Schematic illustration of the reasoning used for deriving neutrino flux limits. In a hadronic source, protons are accelerated to high energies and subsequently may interact with photons or other protons, so that neutrons and neutrinos are created. Whereas the protons are confined within the source by a magnetic field, the neutrons may escape from the source, unless they are turned into a proton by a photohadronic reaction. Neutrons leaving the source decay and thus form the cosmic ray protons. The neutrinos can escape from the source unimpeded.

or other protons, yielding neutrons and pions. The latter decay (if charged) into neutrinos.

Due to the magnetic field in the source (which is required for the particle acceleration) the protons are confined within the source. The neutrons, on the other hand, may escape freely, unless they undergo a photohadronic interaction before leaving the source and are thus turned into a proton. Of course, any produced neutrino can get away unhindered as well.

On their way to Earth, the neutrons decay into protons, so that they can be considered as the origin of the cosmic ray protons observed at Earth. Hence from the cosmic ray spectrum one may infer the original neutron spectrum, and as the neutrons and neutrinos are created by the same processes in the source, this clearly allows to estimate the number of emitted neutrinos. A similar argument is valid for photons.

A quantitative analysis [139] shows that the relation between the muon neutrino and neutron production spectrum $Q$ at the source depends on the spectral index $\alpha$ of the target photon spectrum. For $\alpha=-1$ it is given by

$$
Q_{\nu_{\mu}}\left(E_{\nu_{\mu}}\right)=83.3 Q_{n}\left(25 E_{\nu_{\mu}}\right)
$$


whereas for $\alpha=0$ it has the form

$$
Q_{\nu_{\mu}}\left(E_{\nu_{\mu}}\right)=416 Q_{n}\left(25 E_{\nu_{\mu}}\right) .
$$

Similarly, the bolometric muon neutrino and photon luminosities are related via

$$
L_{\nu_{\mu}}=\left(1+e^{-5 \alpha-5}\right) L_{\gamma}
$$

In order to obtain the "best possible" flux limit, one now assumes a (single source) neutron spectrum of the form $Q_{n} \propto E^{-1} \exp \left(-E / E_{\max }\right)$, and the corresponding muon neutrino spectrum $Q_{\nu_{\mu}}$ is calculated as above. Then the resulting cosmic ray flux $\phi_{\text {cr }}$ observed at the Earth is obtained by summing over all sources and including propagation effects, [139]

$$
\phi_{\mathrm{cr}}(E) \propto \frac{1}{4 \pi} \int_{z_{\min }}^{z_{\max }} M(E, z) \frac{(1+z)^{2}}{4 \pi d_{L}^{2}} \frac{\mathrm{d} N_{\text {source }}}{\mathrm{d} z} Q_{n}((1+z) E),
$$

where $d_{L}$ and $\mathrm{d} N_{\text {source }} / \mathrm{d} z$ denote the luminosity distance [90] and source distribution, respectively, and where $M(E, z)$ takes care of the modifications during the propagation. An analogous relation (with $\phi_{\nu_{\mu}}$ and $Q_{\nu_{\mu}}$ instead of $\phi_{\text {cr }}$ and $Q_{n}$, and with $M(E, z) \equiv 1$ ) holds valid for the muon neutrino flux.

Now the constants of proportionality are chosen so that $\phi_{\mathrm{cr}}$ is as large as possible, but consistent with the (observed) cosmic ray flux limit $\phi_{\text {cr,limit }}$. Then $\phi_{\text {cr }}$ and $\phi_{\text {cr,limit }}$ will coincide at some energy $E_{\text {coincide }}$.

Using the neutrino analogue of Eq. 5.10, one may obtain the corresponding muon neutrino flux $\phi_{\nu_{\mu}}$ at the Earth. Its value at $E_{\text {coincide }}$ can be interpreted as the maximum allowed flux at that energy. Repeating the process for all cutoff energies $E_{\max }$, we thus should get the upper neutrino flux limit.

While this is indeed correct for energies $\gtrsim 10^{4} \mathrm{GeV}$, at lower energies there is the additional constraint that photon and neutrino production are coupled (cf. Eq. 5.9), so that the diffuse gamma radiation background and the neutrino flux must be consistent. If this is taken into account, one obtains the line labeled "MPR, $\tau_{n \gamma<1}$ " in Fig. 5.54. Fig. 5.55 shows this flux limit as a function of the energy and nadir angle, and the corresponding muon event rate as a function of the nadir angle and energy offset.

A slightly different approach is used in [190]. Here, the input neutron spectrum $Q_{n}$ is taken to be of the form $E_{n}^{-2}$ and is normalized to match the cosmic ray flux. The resulting neutrino flux limit must also be a power law 


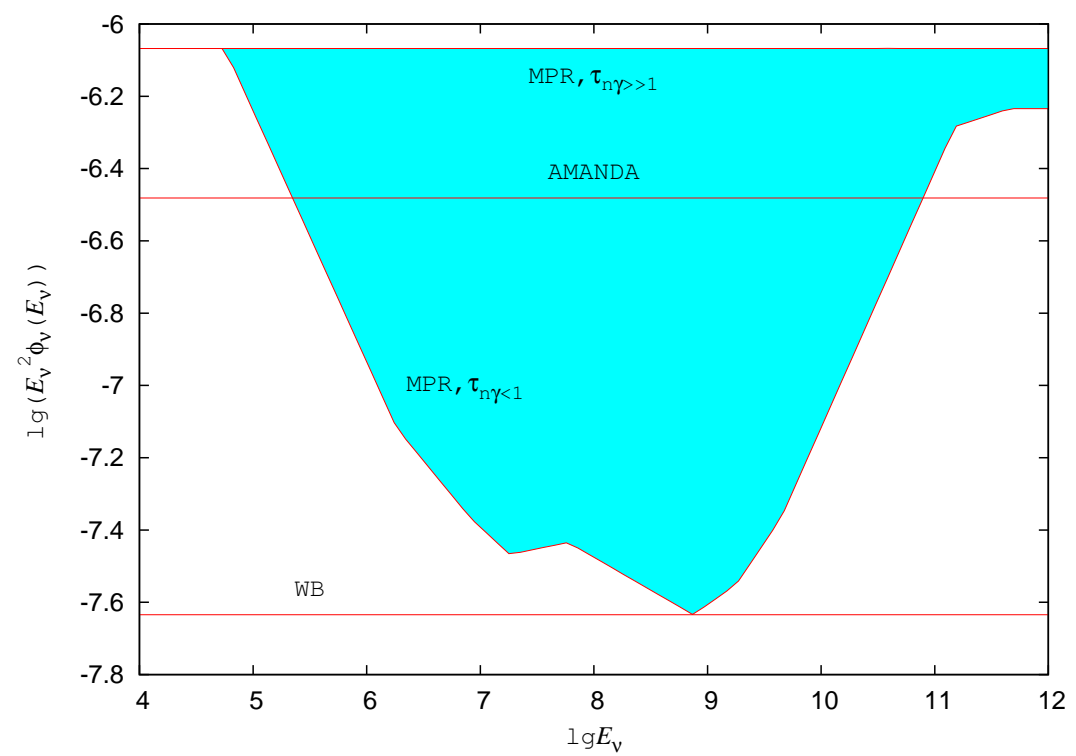

Figure 5.54: Limits for the flux of neutrinos from hadronic sources, as given by Waxman and Bahcall [190] (WB) and by Mannheim, Protheroe and Rachen (MPR) [139] for optically thin $\left(M P R, \tau_{n \gamma<1}\right)$ and thick sources $\left(M P R, \tau_{n \gamma \gg 1}\right)$. Also shown is the current observational flux limit obtained by AMANDA. [2] The shaded area gives the range covered by the MPR flux limits. Flavor oscillations are taken into account.

with spectral index -2 ; it is contained in Fig. 5.54 as the line labeled WB. Fig. 5.56 shows its dependence on the nadir angle and the corresponding event rate.

So far, we have assumed that the sources are optically thin for neutrons. If instead we take them to be optically thick, most of the neutrons don't leave their source and the above analysis would understimate the flux of neutrinos, which still escape from the source unhindered. Hence the only remaining constraint is consistency with the diffuse gamma radiation background. The resulting neutrino flux limit is shown in Fig. 5.54 as the line labeled "MPR, $\tau_{n \gamma \gg 1}$ ". Its dependence on the nadir angle and the corresponding event rate can be found in Fig. 5.57.

Throughout this section, the fundamental prerequisite has been that the neutrinos are created by interactions of accelerated protons. Hence the flux neutrinos from non-hadronic sources isn't covered by the given flux limits and thus could, at least in principle, be arbitrarily high. However, the AMANDA 

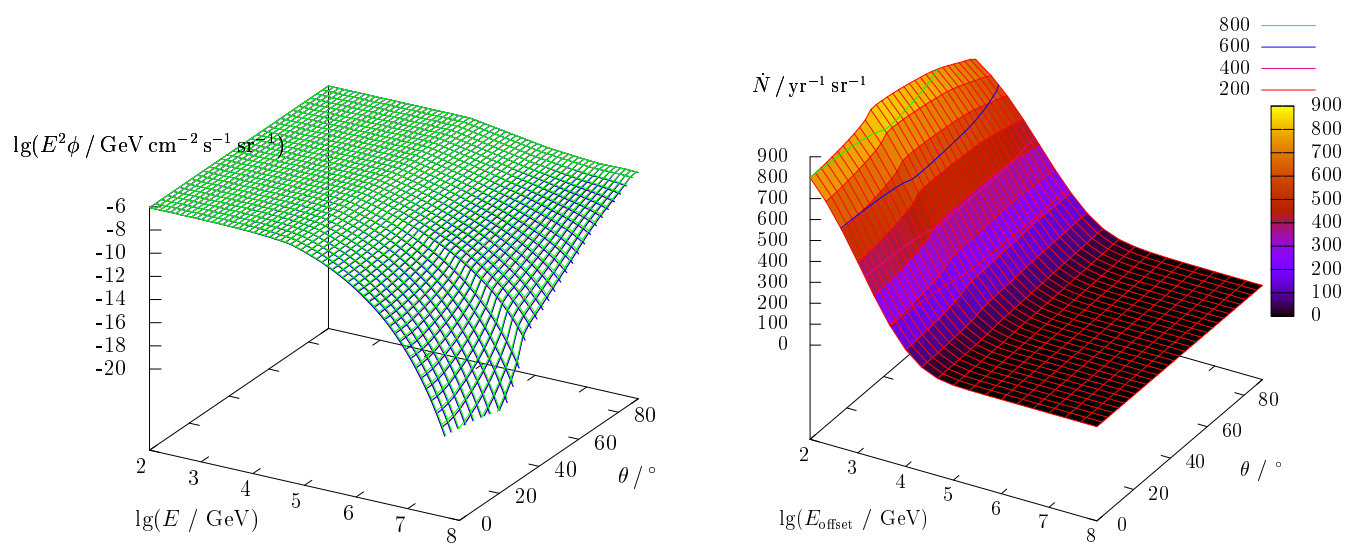

Figure 5.55: Left: Neutrino flux limit for optically thin sources, as given by Mannheim, Protheroe and Rachen. [139] Right: Corresponding muon event rate.
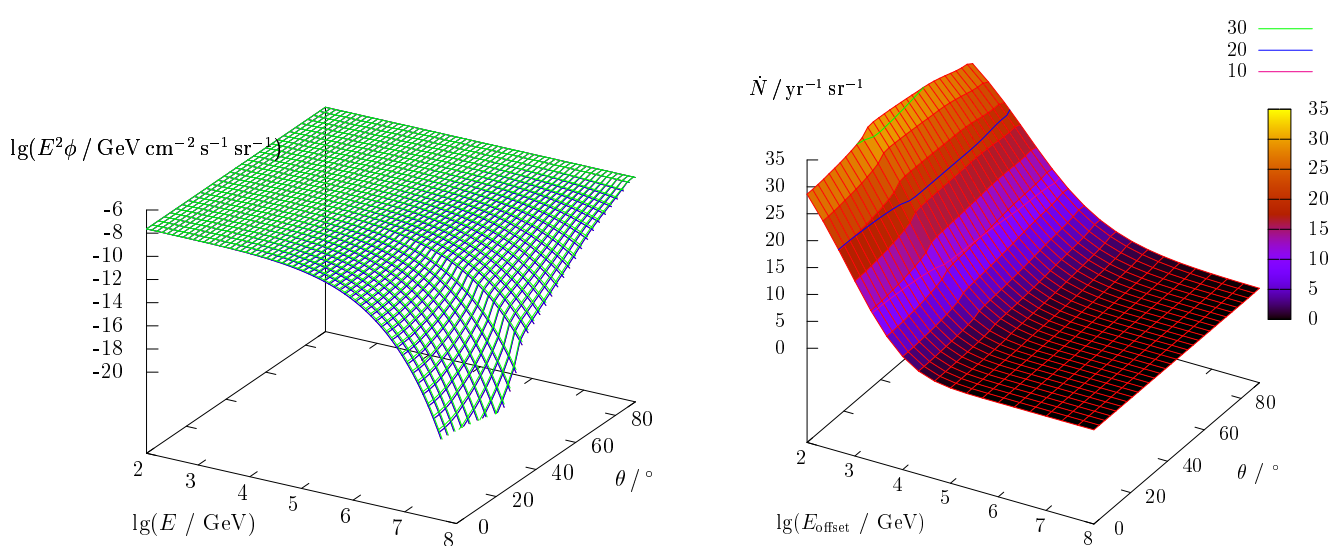

Figure 5.56: Left: Neutrino flux limit, as given by Waxman and Bahcall. [190] Right: Corresponding muon event rate. 

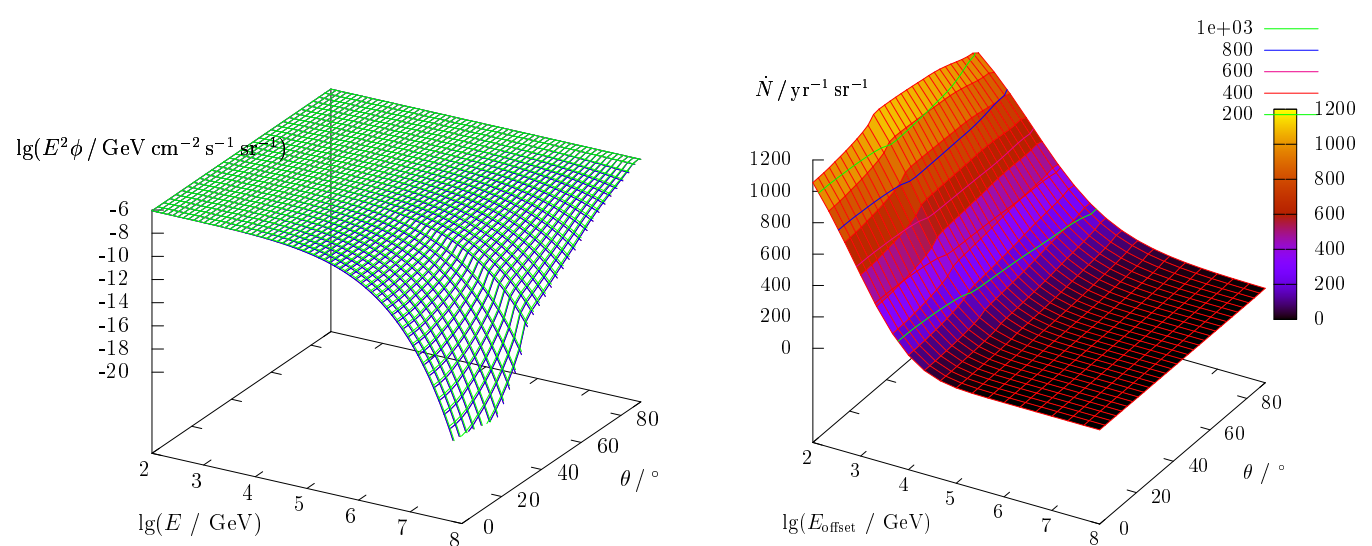

Figure 5.57: Left: Neutrino flux limit for optically thick sources, as given by Mannheim, Protheroe and Rachen. [139] Right: Corresponding muon event rate.

telescope has established an observational upper flux limit, which is already below the MPR limit for optically thick sources [2], as can be seen from the line labeled "AMANDA" in Fig. 5.54. 


\section{Chapter 6}

\section{Tomography of the inner Earth}

One of the most obvious geophysical questions is the one what the structure of the inner Earth looks like. As this cannot be answered by direct inspection, one needs some agency which can cross the Earth and - if it is not produced inside the Earth and thus allows inferences concerning the structure - the propagation of which depends on the density or chemical composition.

Seismic waves are commonly used as the agency, and these allow to establish a fairly robust image of the density profile in the inner Earth. However, neutrinos might be suitable for the task as well, and indeed there are three possible techniques how to use them. Firstly, geoneutrinos produced by the decay of radionuclids in the Earth may provide evidence regarding the chemical composition. [78] Secondly, flavor oscillations of neutrinos from the Sun, a supernova, or a neutrino factory which cross the Earth allow inferences concerning the density profile (cf., e.g., [8, 157, 195]). Finally, absorption and regeneration of an isotropic flux of astrophysical neutrinos inside the Earth might make a tomography possible (cf., e.g., [194, 112, 100, 101, 102, 171]).

In this chapter, we use the last method together with the propagation analysis developed in the preceding chapters in order to outline the prospects and limitations of an inner Earth tomography. Before doing so, however, we first give a brief description of seismic waves and the Preliminary Reference Earth Model, and develop a simple algorithm for calculating an inverse Radon transform. 


\subsection{Preliminary Reference Earth Model}

Man-made holes can reach a depth of at most a few kilometers, so that less than $0.1 \%$ of the inner Earth can be put to a direct investigation. Thus any modelling of the inner Earth structure must rely almost exclusively on indirect methods, the most important being a tomography by means of seismic waves.

Concerning the latter, one has to distinguish between the longitudinal $\mathrm{P}$ ("primary" in the sense of "arriving first") and the transversal S ("secondary") waves. [151] Both are refracted according to Snell's law and both can be reflected at boundaries between regions of different density. Their speed, however, differs. Whereas the speed of $\mathrm{S}$ waves depends on the rigidity modulus $\mu$ of the traversed material only,

$$
v_{\mathrm{S}}=\sqrt{\frac{\mu}{\rho}}
$$

that of $\mathrm{P}$ waves also is influenced by the compressibility modulus $\kappa$,

$$
v_{\mathrm{P}}=\sqrt{\frac{\kappa+\frac{4}{3} \mu}{\rho} .}
$$

From Eqs. 6.1 and 6.2 it is evident that the time required by a wave for crossing the Earth depends on the density and the elastic properties along its path. Hence by considering the travel times of sufficiently many short pulses (generated by, say, an earthquake or artificial explosion) for sufficiently many paths through the Earth one may construct a model of the inner Earth.

For the Preliminary Reference Earth Model (PREM), more than 2000000 $\mathrm{P}$ wave and $250000 \mathrm{~S}$ wave travel times from about 26000 events were used. In addition, long period surface wave data was taken into account, and the mass, radius, and moment of inertia were employed as model constraints.

Broadly speaking, the Earth as seen by the PREM consists of a solid inner core ranging from the middle of the Earth to a radius of $1221.5 \mathrm{~km}$, a liquid outer core from 1221.5 to $3480 \mathrm{~km}$, the (again solid) mantle with radii between 3480 and roughly $6347 \mathrm{~km}$, and finally the crust from the top of the mantle up to the surface. Here, the liquidness of the outer core can be deduced from the observational fact that this region transmits $\mathrm{P}$, but no $\mathrm{S}$ waves. This suggests that the rigidity modulus vanishes, which is a generic feature of all liquids as opposed to solid bodies. 

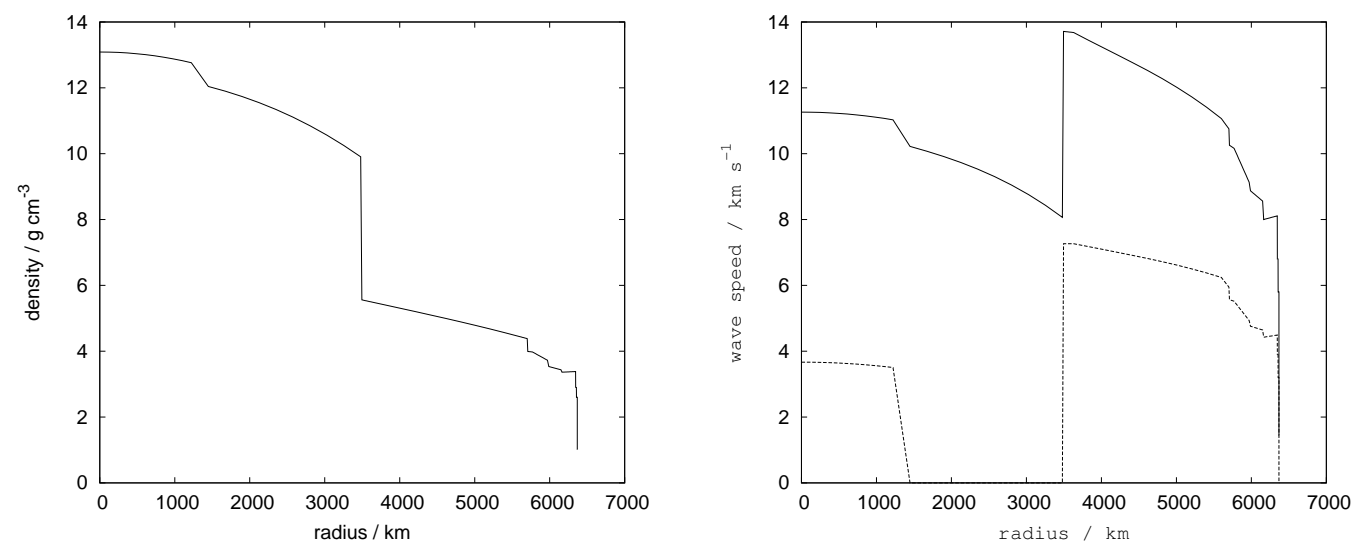

Figure 6.1: Left: Density as a function of the radius for the Preliminary Reference Earth Model. Right: The corresponding P (solid) and S wave speed (dashed line) as a function of the radius. Between 6151 and $6346.6 \mathrm{~km}$ the speeds are anisotropic, but effective isotropic values are shown.

More precisely, the density distribution $\rho_{\text {PREM }}$ according to the PREM can be parametrized as [69]

$$
\frac{\rho_{\text {PREM }}(r)}{1 \mathrm{~g} / \mathrm{cm}^{3}}= \begin{cases}\text { inner core: } & (0 \mathrm{~km} \leqslant r<1221.5 \mathrm{~km}) \\ 13.0885-8.8381 x^{2} & \\ \text { outer core: } & (1221.5 \mathrm{~km} \leqslant r<3480 \mathrm{~km}) \\ 12.5815-1.2638 x-3.6426 x^{2}-5.5281 x^{3} & \\ \text { mantle: } & (3480 \mathrm{~km} \leqslant r<5701 \mathrm{~km}) \\ 7.9565-6.4761 x+5.5283 x^{2}-3.0807 x^{3} & (5701 \mathrm{~km} \leqslant r<5771 \mathrm{~km}) \\ 5.3197-1.4836 x & (5771 \mathrm{~km} \leqslant r<5971 \mathrm{~km}) \\ 11.2494-8.0298 x & (5971 \mathrm{~km} \leqslant r<6151 \mathrm{~km}) \\ 7.1089-3.8045 x & (6151 \mathrm{~km} \leqslant r<6346.6 \mathrm{~km}) \\ 2.6910+0.6924 x & \\ \text { crust: } & (6346.6 \mathrm{~km} \leqslant r<6356 \mathrm{~km}) \\ 2.900 & (6356 \mathrm{~km} \leqslant r<6368 \mathrm{~km}) \\ 2.600 & (6368 \mathrm{~km} \leqslant r<6371 \mathrm{~km}) \\ 1.020 & \end{cases}
$$

where $x$ denotes the ratio of the distance $r$ from the center and the radius $R_{\text {Earth }}=6371 \mathrm{~km}$. The left panel of Fig. 6.1 illustrates this density profile, and the right panel gives the corresponding $\mathrm{P}$ and $\mathrm{S}$ wave speeds [69]. The column number density as a function of the nadir angle is shown for the PREM density profile in Fig. 6.2.

There are some noteworthy points concerning the given parametrization: 


\begin{tabular}{|l|l|lll|}
\hline region & predominant elements & $\langle\mathbf{Z}\rangle$ & $\langle\mathbf{A}\rangle$ & $\langle\mathbf{Z}\rangle /\langle\mathbf{A}\rangle$ \\
\hline continental crust & $\mathrm{O}(47.3 \%), \mathrm{Si}(27.6 \%), \mathrm{Al}(78.4 \%)$ & 10.5 & 21.2 & 0.495 \\
mantle & $\mathrm{O}(44.8 \%), \mathrm{Si}(21.5 \%), \mathrm{Mg}(22.8 \%)$ & 10.4 & 21.0 & 0.496 \\
core & $\mathrm{Fe}(79.4 \%), \mathrm{Si}(7.4 \%), \mathrm{Ni}(4.9 \%)$ & 22.0 & 46.8 & 0.471 \\
\hline
\end{tabular}

Table 6.1: Chemical composition of the Earth. The mass percentages of the most abundant elements, the average atomic and mass numbers, and their ratios are given. The data has been taken from [30].

Firstly, the density of $1.02 \mathrm{~g} / \mathrm{cm}^{3}$ in the outermost region refers to water. Hence the depth of this region varies from location to location, and the given value of $3 \mathrm{~km}$ should be regarded as an average only. Secondly, the depth of the mantle-crust boundary depends on the location as well; its average is $7 \mathrm{~km}$ below the oceans and $40 \mathrm{~km}$ below the continents. Thirdly, as due to its rotation the Earth is an ellipsoid rather than perfect sphere, the region boundaries should be scaled with the ratio of the actual radius for the considered latitude and $R_{\text {Earth }}$. Fortunately, this factor is $\lesssim 1.003$ and hence can be disregarded within this work. The most notable feature of the density profile, however, obviously is the core-mantle boundary at $r=3480 \mathrm{~km}$, where the density changes by a factor of almost 2 and below which the speed of $\mathrm{S}$ waves vanishes until the radius reaches the inner core.

The preceding paragraphs should have made clear that especially near the Earth surface, the PREM should be taken cum grano salis, as it describes an anisotropic structure in terms of an isotropic model. Apart from this, however, the parametrization used in the PREM turns out to be quite adequate, although the use of single cubic polynomials in the lower mantle and core is somewhat unsatisfying. [115]

A description of the inner Earth would be incomplete without giving its chemical composition. For the sake of this work a crude distinction between the compositions of crust, mantle, and core is sufficient; this is found in Table 6.1. A more detailed analysis for the mantle and core can be found in $[12]$.

\subsection{Inverse Radon transform}

Absorption (and regeneration) of high energy neutrinos may provide a new window into the structure of the inner Earth. The underlying principle is straightforward [194]: For some given initial spectrum, the final spectrum 


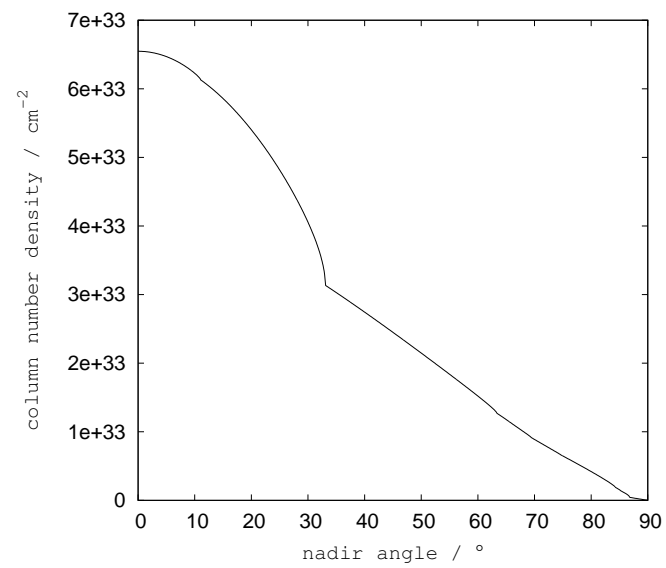

Figure 6.2: Column number density as a function of the nadir angle for the density profile given by the Preliminary Reference Earth Model.

(and thus the event rate) after crossing the Earth depends on the amount of matter crossed on the way. In addition the column number density is a unique function of the angle of observation. Throughout this chapter we assume that the Earth is spherically symmetric.

Hence if the initial neutrino spectrum is known, one may use the observed angular dependence of the event rate to obtain the column number density, and the only remaining task is to figure out the corresponding density inside the Earth.

We'll start with the latter. To this end, we first note that the Radon transform $\check{f}(p, \boldsymbol{\xi})$ of some function $f$ in $\mathbb{R}^{n}$ is defined as [62]

$$
\check{f}(p, \boldsymbol{\xi}) \equiv \int f(\boldsymbol{\xi}) \delta(p-\boldsymbol{\xi} \boldsymbol{x}) \mathrm{d}^{n} x,
$$

where $p$ and $\boldsymbol{\xi}$ denote the distance from the origin and a unit vector, respectively. From an intuitive point of view, this means that $\check{f}(p, \boldsymbol{\xi})$ constitutes the integral of $f(\boldsymbol{x})$ over the $(n-1)$-dimensional manifold with normal vector $\boldsymbol{\xi}$ and distance $p$ from the origin. In other words, for the case of $\mathbb{R}^{2}$ and $\mathbb{R}^{3}$, the Radon transform is an integral over a line and a plane, respectively (cf. Fig. 6.3).

Accordingly, if $f$ is a density, its two-dimensional Radon transform is a column number density. Hence the connection between a tomography of the Earth and the Radon transform is obvious: Knowing the column density 

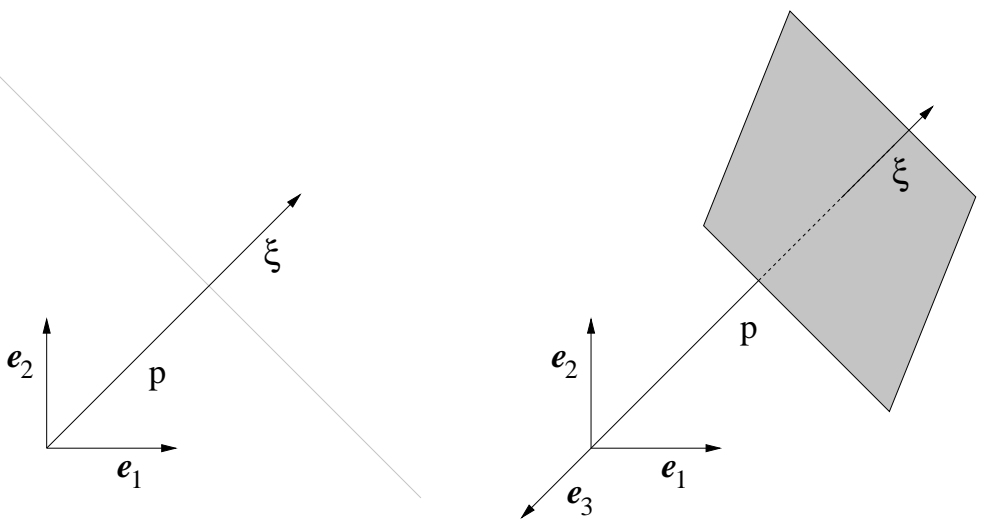

Figure 6.3: Illustration of the Radon transform $\check{f}(\boldsymbol{\xi}, p)$. Left: In $\mathbb{R}^{2}$, the Radon transform corresponds to an integration over the line with normal vector $\boldsymbol{\xi}$ and distance $p$ from the origin. Right: In $\mathbb{R}^{3}$, it corresponds to an integration over the plane with normal vector $\boldsymbol{\xi}$ and distance $p$ from the origin.

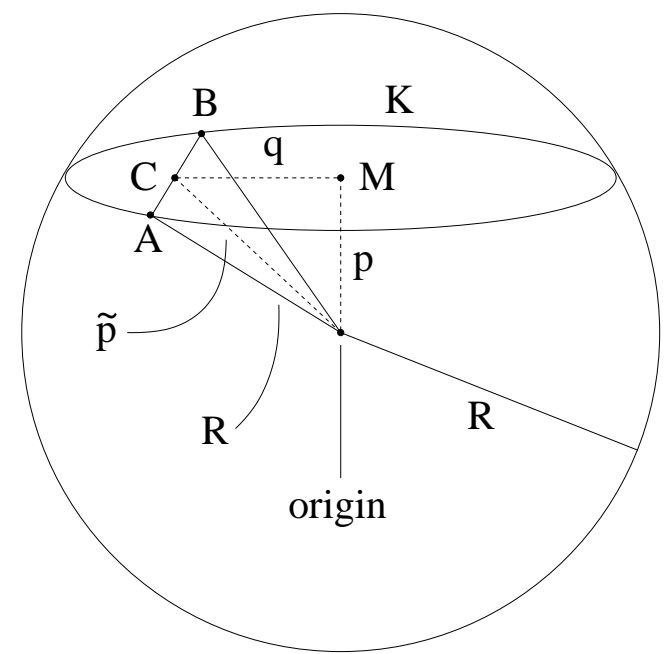

Figure 6.4: Notations used in deriving the formula for the inverse Radon transform. The various quantities are explained in the main text. 
under all angles of incidence for some cross section of the Earth through its center, one may obtain the density profile from its inverse Radon transform.

Here, the latter is given by [62]

$$
f(\boldsymbol{x})=-\frac{1}{2 \pi^{2}} \int_{0}^{\pi} \mathrm{d} \phi \int_{-\infty}^{\infty} \mathrm{d} p \frac{1}{p-\boldsymbol{\xi} \boldsymbol{x}} \frac{\partial \check{f}(p, \boldsymbol{\xi})}{\partial p}
$$

As we take the Earth to be spherically symmetric, the density depends on the distance $r$ from the origin only. In addition, we know that the density vanishes for distances exceeding the Earth radius $R$.

Alas, even with these simplifications, Eq. 6.4 remains, innocent-looking though it might be, quite intractable when employed for concrete calculations. We therefore compute the inverse transform by means of a method that might seem like a detour, namely we consider the three-dimensional Earth rather than one of its two dimensional cross sections.

Hence let us consider a spherically symmetric function $f(\boldsymbol{x})=f(|\boldsymbol{x}|)$ in $\mathbb{R}^{3}$ vanishing for radii $r \equiv|\boldsymbol{x}| \leqslant R$. Then the Radon transform $\check{f}$ of $f$ constitutes the integral over a cross section of the sphere with radius $R$ centered about the origin. It mustn't be confused with the two-dimensional Radon transform for a cross section through the origin, which we shall denote by $\tilde{\tilde{f}}$. Due to the spherical symmetry both transforms don't depend on the normal vector $\boldsymbol{\xi}$, hence we omit the corresponding argument.

We now turn to a cross section with distance $p$ from the origin. Its boundary $K$ beyond which $f$ vanishes must be a circle. Let $M$ be the center of that circle, $A$ and $B$ points of $K, C$ the center of the secant through $A$ and $B$, and $q$ the (signed) distance between $C$ and $M$. Finally, let $\tilde{p}$ stand for the distance from $C$ to the origin. Fig. 6.4 illustrates these notations.

As $A, B, C$, and the origin obviously lie in a common plane, one may easily see that the column density of the secant through $A$ and $B$ is just $\check{\tilde{f}}(\tilde{p})$. In addition, the radius of $K$ has the value $\sqrt{R^{2}-p^{2}}$, and $\tilde{p}$ may be expressed in terms of $p$ and $q$ as $\tilde{p}=\sqrt{p^{2}+q^{2}}$. Thus $\check{f}(p)$ (i.e. the integral over the cross section) may be written as

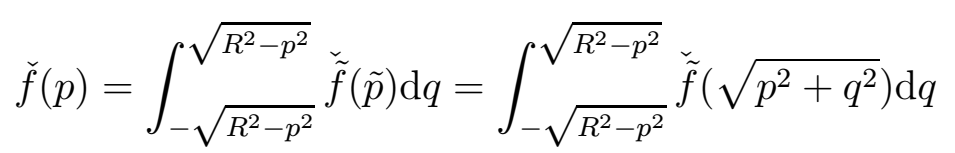

But the inverse Radon transform in $\mathbb{R}^{3}$ may be computed by means of the 
formula [62]

$$
f(\boldsymbol{x})=-\frac{1}{8 \pi^{2}} \Delta_{\boldsymbol{x}} \int_{|\boldsymbol{\xi}|=1} \check{f}(\boldsymbol{\xi} \boldsymbol{x}, \boldsymbol{\xi}) \mathrm{d}^{2} \xi
$$

where as before we may drop the second argument of $\check{f}$. Together with the relation

$$
\Delta_{\boldsymbol{x}} g(\boldsymbol{\xi} \boldsymbol{x})=\boldsymbol{\xi}^{2} g^{\prime \prime}(\boldsymbol{\xi} \boldsymbol{x})
$$

for an arbitrary function $g$ we therefore have

$$
f(\boldsymbol{x})=-\frac{1}{8 \pi^{2}} \int_{|\boldsymbol{\xi}|=1} \underbrace{\boldsymbol{\xi}^{2}}_{=|\boldsymbol{\xi}|^{2}=1} \check{f}^{\prime \prime}(\boldsymbol{\xi} \boldsymbol{x}) \mathrm{d}^{2} \xi=-\frac{1}{8 \pi^{2}} \int_{|\boldsymbol{\xi}|=1} \check{f}^{\prime \prime}(\boldsymbol{\xi} \boldsymbol{x}) \mathrm{d}^{2} \xi .
$$

As $f$ depends on the radius $r$ only, we may assume without loss of generality that $\boldsymbol{x}$ points in $\mathrm{z}$ direction. Hence, using spherical coordinates for the integration, we have $\boldsymbol{\xi} \boldsymbol{x}=r \mu$ (where $\mu \equiv \cos \theta)$, and with $u \equiv r \mu$ we get

$$
f(r)=-\frac{1}{8 \pi^{2}} \int_{0}^{2 \pi} \mathrm{d} \phi \int_{-1}^{1} \mathrm{~d} \mu \check{f}^{\prime \prime}(r \mu)=-\frac{1}{4 \pi r} \int_{-r}^{r} \mathrm{~d} u \check{f}^{\prime \prime}(u) .
$$

The remaining integral is trivial, and we finally arrive at the remarkably simple formula

$$
f(r)=-\frac{1}{4 \pi r}\left[\check{f}^{\prime}(r)-\check{f}^{\prime}(-r)\right]
$$

Hence if $\check{\tilde{f}}$ is known, the inverse Radon transform can be obtained from Eqs. 6.5 and 6.6. For the sake of illustration, consider the two-dimensional Radon transform

$$
\check{\tilde{f}}(\tilde{p})=\left\{\begin{array}{ll}
1-\tilde{p}^{2} & (\tilde{p} \leqslant 1) \\
0 & (\text { otherwise })
\end{array} .\right.
$$

From Eq. 6.5 we obtain

$$
\check{f}(p)=\int_{-\sqrt{1-p^{2}}}^{\sqrt{1-p^{2}}}\left(1-\left[p^{2}+q^{2}\right]\right) \mathrm{d} q=\frac{4}{3}\left(1-p^{2}\right)^{3 / 2},
$$


and by means of differentiating $\check{f}(p)$ and employing Eq. 6.6 we get

$$
f(r)=-\frac{1}{4 \pi r}\left[-4 r \sqrt{1-r^{2}}-\left(-4(-r) \sqrt{1-(-r)^{2}}\right)\right]=\frac{2}{\pi} \sqrt{1-r^{2}} .
$$

Indeed, Radon transforming this $f(r)$ yields the original Radon transform again (cf. example 4 on page 63 of [62]).

We can still simplify the formulae a little bit. To this end, we note that due to the spherical symmetry, $\tilde{\tilde{f}}(-p)=\tilde{\tilde{f}}(p)$ and thus

$$
\check{f}(-p)=\check{f}(p)
$$

must hold valid, and Eqs. 6.5 and 6.6 can be rewritten as

$$
\check{f}(p)=2 \int_{0}^{\sqrt{R^{2}-p^{2}}} \check{\tilde{f}}\left(\sqrt{p^{2}+q^{2}}\right) \mathrm{d} q
$$

and

$$
f(r)=-\frac{1}{2 \pi r} \check{f}^{\prime}(r)
$$

respectively. Evidently, the inverse Radon transform can be performed analytically only if the integral on the right hand side of Eq. 6.8 is known, which in general won't be the case. But we may take advantage of the fact that because of the limited statistics, the precise form of the column density won't be known for a tomography with high energy neutrinos. Hence there is nothing wrong with using a suitable approximation. In particular, we may choose a piecewise parabolic approximation, for which an analytic solution exists, as we'll show now.

So let us consider a two-dimensional Radon transform of the form

$$
\check{\tilde{f}}(\tilde{p})= \begin{cases}A+B \tilde{p}+C \tilde{p}^{2}+D \tilde{p}^{3} & (\tilde{p} \in[a, b[) \\ 0 & \text { (otherwise) }\end{cases}
$$

where without real loss of generality we may take $a$ and $b$ to be non-negative (cf. Eq. 6.7). When computing $\check{f}(p)$, we have to distinguish the three cases 
$0<p<a, a<p<b$ and $p>b$. For $0<p<a$, we obtain from Eq. 6.5

$$
\begin{array}{r}
\check{f}(p)=2 \int_{\sqrt{a^{2}-p^{2}}}^{\sqrt{b^{2}-p^{2}}}\left(A+B\left(p^{2}+q^{2}\right)^{\frac{1}{2}}+C\left(p^{2}+q^{2}\right)+D\left(p^{2}+q^{2}\right)^{\frac{3}{2}}\right) \mathrm{d} q \\
=2\left[\left(A+C p^{2}\right) q+\frac{C q^{3}}{3}+\sqrt{p^{2}+q^{2}}\left(\frac{\left(4 B+5 D p^{2}\right) q}{8}+\frac{D q^{3}}{4}\right)\right. \\
\left.+\frac{p^{2}\left(4 B+3 D p^{2}\right) \ln \left(q+\sqrt{p^{2}+q^{2}}\right)}{8}\right]_{\sqrt{a^{2}-p^{2}}}^{\sqrt{b^{2}-p^{2}}}
\end{array}
$$

whereas for $a<p<b$ we completely analogously get

$$
\begin{aligned}
\check{f}(p)=2\left[\left(A+C p^{2}\right) q+\frac{C q^{3}}{3}\right. & +\sqrt{p^{2}+q^{2}}\left(\frac{\left(4 B+5 D p^{2}\right) q}{8}+\frac{D q^{3}}{4}\right) \\
& \left.+\frac{p^{2}\left(4 B+3 D p^{2}\right) \ln \left(q+\sqrt{p^{2}+q^{2}}\right)}{8}\right]_{0}^{\sqrt{b^{2}-p^{2}}} .
\end{aligned}
$$

Finally, for $p>b$, the Radon transform vanishes identically,

$$
\check{f}(p) \equiv 0 .
$$

Now let us define the functions $G(x, p)$ and $H(p)$ as

$$
\begin{aligned}
\frac{1}{2} G(x, p) \equiv C p & \sqrt{x^{2}-p^{2}}+x \cdot \frac{D p \sqrt{x^{2}-p^{2}}}{2}+\frac{3 D p^{3} \ln \left(x+\sqrt{x^{2}-p^{2}}\right)}{4} \\
& +\frac{p\left(4 B+3 D p^{2}\right) \ln \left(x+\sqrt{x^{2}-p^{2}}\right)}{4} \\
& +\frac{1}{\sqrt{x^{2}-p^{2}}}\left(-p\left(A+C p^{2}\right)-\frac{p^{3}\left(4 B+3 D p^{2}\right)}{8\left(x+\sqrt{x^{2}-p^{2}}\right)}\right. \\
& \left.-x \cdot \frac{p\left(4 B+5 D p^{2}\right)}{8}\right)
\end{aligned}
$$

and

$$
\frac{1}{2} H(p) \equiv p\left(\frac{4 B+3 D p^{2}}{8}+\frac{\left(2 B+3 D p^{2}\right) \ln p}{2}\right) .
$$


Using Eq. 6.9 and Eqs. 6.10 6.14, we may write $f(r)$ in the succint form

$$
f(r)=-\frac{1}{2 \pi r} \times \begin{cases}G(b, r)-G(a, r) & (r<a) \\ G(b, r)-H(r) & (a<r<b) . \\ 0 & (b<r)\end{cases}
$$

While the function $H(p)$ is well-behaved, $G(x, p)$ contains the term

$$
\begin{array}{r}
Q(p, x) \equiv \frac{1}{\sqrt{x^{2}-p^{2}}}\left(-p\left(A+C p^{2}\right)-\frac{p^{3}\left(4 B+3 D p^{2}\right)}{8\left(x+\sqrt{x^{2}-p^{2}}\right)}\right. \\
\left.-\frac{x p\left(4 B+5 D p^{2}\right)}{8}\right)
\end{array}
$$

which clearly diverges for $p \longrightarrow x$. The existence of such a divergence can be understood intuitively when taking into account that due to its finite support, $\tilde{\tilde{f}}$ is discontinuous at the support boundaries. As the Radon transform basically is an integral, we may paraphrase this statement by saying that at $a$ and $b$ an infinitesimal change in the integration range leads to a finite change in the integral value. Hence the integrand (i.e. the function $f$ ) must resemble a delta function, which implies the existence of a divergence. We'll comment on its (un)importance in a moment.

First, however, let us make a slight generalization. So far, we have confined ourselves to a single interval. However, we might as well use $N$ such intervals $\left[a_{i}, a_{i+1}\right]$, where the boundaries $a_{i}$ are assumed to be monotonically increasing with $a_{1}=0$ and $a_{N+1}=R$. In addition, we may define $N$ corresponding polynomial Radon transforms as

$$
\check{\tilde{f}}_{i}(\tilde{p}) \equiv\left\{\begin{array}{ll}
A_{i}+B_{i} \tilde{p}+C_{i} \tilde{p}^{2}+D_{i} \tilde{p}^{3} & \left(\tilde { p } \in \left[a_{i}, a_{i+1}[)\right.\right. \\
0 & \text { (otherwise) }
\end{array},\right.
$$

where the coefficients $A_{i}, B_{i}, C_{i}$ and $D_{i}$ are chosen so that

$$
A_{i}+B_{i} a_{i+1}+C_{i} a_{i+1}^{2}+D_{i} a_{i+1}^{3}=A_{i+1}+B_{i+1} a_{i+1}+C_{i+1} a_{i+1}^{2}+D_{i+1} a_{i+1}^{3} .
$$

Then the Radon transform

$$
\check{\tilde{f}}(\tilde{p}) \equiv \sum_{i=1}^{N} \check{\tilde{f}}_{i}(\tilde{p})
$$


has a continuous piecewise polynomial representation, and as both integration and applying a Laplace operator are linear operations, we may thus immediately deduce from Eqs. 6.8, 6.6 and Eq. 6.15 that

$$
f(r)=\sum_{i=1}^{N}\left(-\frac{1}{2 \pi r}\right) \times \begin{cases}G_{i}\left(a_{i+1}, r\right)-G_{i}\left(a_{i}, r\right) & \left(r<a_{i}\right) \\ G_{i}\left(a_{i+1}, r\right)-H_{i}(r) & \left(a_{i}<r<a_{i+1}\right) \\ 0 & \text { (otherwise) }\end{cases}
$$

where the $G_{i}$ and $H_{i}$ are defined as in the case of a single interval, but with the polynomial coefficients for the $i$-th interval. Now consider the divergent term $Q_{i}$ defined analogously to Eq. 6.16. We have

$$
\begin{aligned}
& \lim _{p \longrightarrow x} Q_{i}(x, p) \\
& \quad=\lim _{p \longrightarrow x}\left(\frac{1}{\sqrt{x^{2}-p^{2}}}\left[-p\left(A_{i}+C_{i} p^{2}\right)-\frac{p^{3}\left(4 B_{i}+3 D_{i} p^{2}\right)}{8\left(x+\sqrt{x^{2}-p^{2}}\right)}-\frac{x p\left(4 B_{i}+5 D_{i} p^{2}\right)}{8}\right]\right) \\
& \quad=\lim _{p \longrightarrow x}\left(\frac{1}{\sqrt{x^{2}-p^{2}}}\left[-x\left(A_{i}+C_{i} x^{2}\right)-\frac{x^{3}\left(4 B_{i}+3 D_{i} x^{2}\right)}{8 x}-\frac{x^{2}\left(4 B_{i}+5 D_{i} x^{2}\right)}{8}\right]\right) \\
& \quad=\lim _{p \longrightarrow x}\left(\frac{1}{\sqrt{x^{2}-p^{2}}}\left[-x\left(A_{i}+B_{i} x+C_{i} x^{2}+D_{i} x^{3}\right)\right]\right)=-\lim _{p \longrightarrow x}\left(\frac{x \tilde{\tilde{f}}_{i}(x)}{\sqrt{x^{2}-p^{2}}}\right)
\end{aligned}
$$

and thus from Eq. 6.17

$$
\lim _{r \longrightarrow a_{i+1}} Q_{i}(x, r)=\lim _{r \longrightarrow a_{i+1}} Q_{i+1}(x, r) .
$$

The divergences mentioned above occur at the boundaries between neighbouring intervals. However, it is easy to see from Eqs. 6.18 and 6.19 that at each such boundary $a_{i}$ there is a divergence from $Q_{i}$ and one from $Q_{i+1}$, which have the same absolute value, but opposite sign, and accordingly cancel. Hence, as should have been expected, the divergences don't contribute to the inverse Radon transform.

\subsection{Weighing the Earth}

The main obstacle to an application of the formalism developed in Sect. 6.2 is formed, not unexpectedly, by the statistical fluctuations of the detected event rate, which have two profound related consequences: Firstly, assuming 
a Poisson deviation for the events, any number $N$ of events has an uncertainty $\sqrt{N}$, which proves to be substantial. Secondly, this implies that for any realistic spectrum, in the foreseeable future one will presumably be limited to considering a binned (rather than differential) event rate with a rather low number $n$ (10 or 20 , say) of bins for the nadir angle. Hence we have to cope with a limited angular resolution. In the following, we choose the boundaries

$$
\theta_{i} \equiv \arccos (1-(i-1) \Delta) \quad(i=1,2, \ldots, n+1)
$$

with $\Delta \equiv 1 / n$, so that the corresponding bins for $\cos \theta$ are of equal length $\Delta$ (cf. the end of Sect. 5.2).

In practice, one might additionally have to take a limited energy resolution of the detector into account. However, within the framework of our generic detector introduced in Sect. 4.2, we considered a perfect energy resolution, and for the sake of simplicity, we continue with this assumption. If required, an uncertainty in the energy resolution could be included in the upcoming analysis by appropriately changing the statistical event rate fluctuations.

Of course, when performing a tomography of the Earth, one needs to know (at least in principle) the column number density and thus the number $N$ of events at all nadir angles $\theta$. Alas, as should be clear from the above, we just have $n$ values $N_{i}$, and, even worse, these are to be understood as an average over the respective nadir angle bin. But there obviously is a wealth of possible distributions $N(\theta)$ consistent with the $N_{i}$. For example, the completely unrealistic distribution

$$
N_{\text {unrealistic }}(\theta) \equiv \frac{1}{2 \pi} \sum_{i=1}^{n} N_{i} \delta\left(\cos \theta-\cos \theta_{i}\right)
$$

would suit the data perfectly well. Hence we are forced to make some judicious choice concerning some recipe for constructing a continuous distribution of events from the $N_{i}$. As a compromise between the desire to avoid any assumptions about the inner Earth and the need to ensure that the event distribution is reasonable, we adopt a fairly agnostic point of view and take $N(\theta)$ to be a piecewise linear function of $\theta$.

To be more specific, we assume that at the nadir angle $\tilde{\theta}_{k}$ defined as

$$
\tilde{\theta}_{k} \equiv \arccos \left(\frac{1}{2}\left(\cos \theta_{k+1}+\cos \theta_{k}\right)\right)
$$



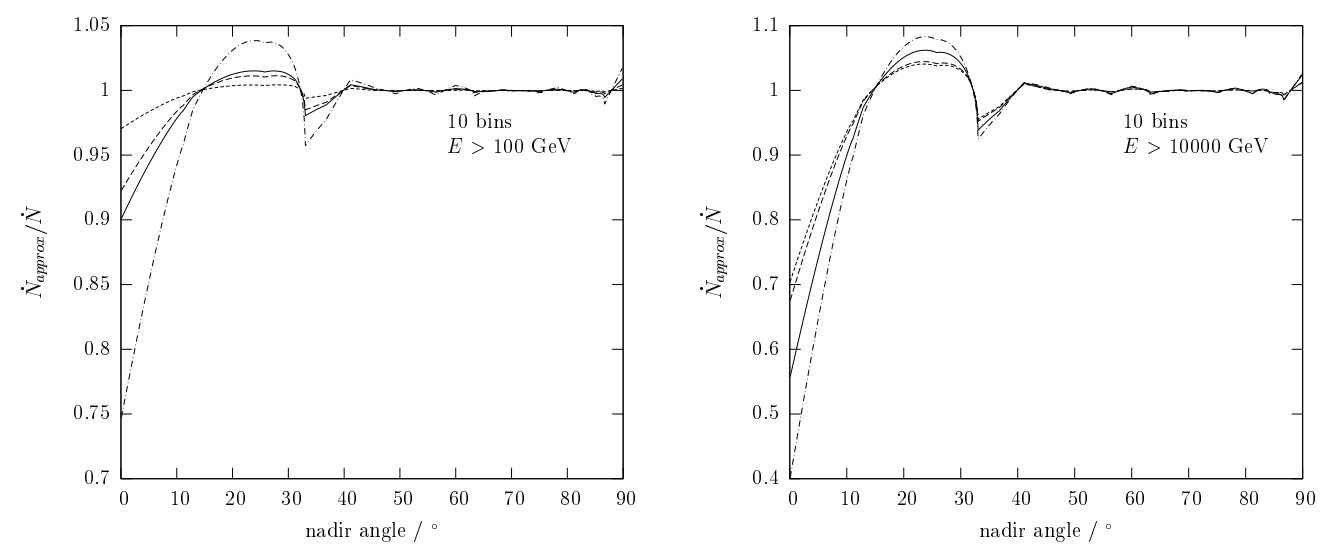

Figure 6.5: Left: Ratio of the approximate event rate constructed from the binned event rates and the true event rate as a function of the nadir angle. The upper (dashed) and lower MPR flux limit (dotted), Gen(0.5,1.5) (dot-dashed), and Gen $(1.0,0.5)$ (solid line) are assumed as the initial neutrino spectrum. 10 nadir bins are used, and the energy offset for the detector is taken to be $100 \mathrm{GeV}$. Right: The same as the left panel, but for an energy offset of $10^{4} \mathrm{GeV}$.

the event distribution is equal to the average value within the respective bin, $N\left(\tilde{\theta}_{i}\right)=N_{i} /(2 \pi \Delta)$, and that the distribution at any other nadir angle is obtained from linear interpolation or extrapolation of these values. Alas, this prescription does not guarantee that the resulting event distribution actually is consistent with the binned events $N_{i}$.

We remedy the situation by appropriately changing the event rate at the mid points $\hat{\theta}_{k} \equiv\left(\theta_{k}+\theta_{k+1}\right) / 2$ of all the bins and assuming that the event rate is piecewise linear between the bin boundaries and mid points. Fig. 6.5 shows the ratio of the approximate event rate thus obtained and the true event rate for two energy offsets and the lower and upper MPR flux limit, $\operatorname{Gen}(0.5,1.5)$, and Gen(1.0,0.5) (cf. page 93 for the nomenclature) as the initial neutrino spectrum. Here and in the following, the event rates are computed under the assumption that the density profile is given by the Preliminary Reference Earth Model.

One sees that the accuracy is acceptable for nadir angles above $40^{\circ}$, but rather low for smaller angles. This is due to the fact that the size of the chosen nadir angle bins decreases with the angle. For example, taking $n=10$, we have $\theta_{1}=0^{\circ}$ and $\theta_{2}=25.8^{\circ}$, so that about one quarter of the clearly nonlinear column number density is described by a single line. 
To further illustrate the problem, imagine that we want to measure the number of events in a bin covering the nadir angles from $0^{\circ}$ to $1^{\circ}$ with an accuracy of, say, $15 \%$. Assuming that the events follow a Poisson distribution, we then need about 44 events in this bin. However, the solid angle $\Omega\left(0^{\circ}, 1^{\circ}\right)$ corresponding to the bin has the value

$$
\Omega\left(0^{\circ}, 1^{\circ}\right)=2 \pi\left(\cos \left(0^{\circ}\right)-\cos \left(1^{\circ}\right)\right) \approx 2 \pi \times 1.52 \times 10^{-4}
$$

so that under the assumption of an isotropic flux the total number $N_{2 \pi}$ of events from neutrinos with nadir angles between $0^{\circ}$ and $90^{\circ}$ must be of the order

$$
N_{2 \pi}=\frac{2 \pi}{\Omega\left(0^{\circ}, 1^{\circ}\right)} \times 44 \approx 2.9 \times 10^{5}
$$

so that an inaccuracy of $15 \%$ or even less for the neutrino flux obtained from the event rate for all nadir angles, as assumed in [171], appears to be fairly optimistic.

Now let us assume for the moment that the binned event numbers can be measured perfectly. Then the column number density can be reconstructed without any further imprecision from the event distribution obtained for the given $N_{i}$. Results of such an analysis are shown in the left panel of Fig. 6.6. Having obtained the column number density, one may subsequently use the formalism developed in Sect. 6.2 to get the corresponding density distribution inside the Earth. The densities thus computed are given in the right panel of Fig. 6.6. Note that the results for tauon events would be practically the same.

The piece of good news apparent from Fig. 6.6 is that the column number density can be retrieved fairly reliably for nadir angles greater than about $40^{\circ}$, so that for the density reasonable values are obtained at radii exceeding $4500 \mathrm{~km}$. Alas, for smaller angles the true and reconstructed column number densities differ significantly, and this implies an immense deviation of the reconstructed from the real density profile.

In accordance with expectation, things improve with an increasing number of bins, as the real event distribution is better approximated. This is illustrated by Fig. 6.7, which shows the nadir column density and density obtained from an ideal detection in 20 nadir angle bins. In the limit of very large numbers of nadir angle bins, the original Preliminary Reference Model is faithfully reproduced, as can be seen from Fig. 6.8. 

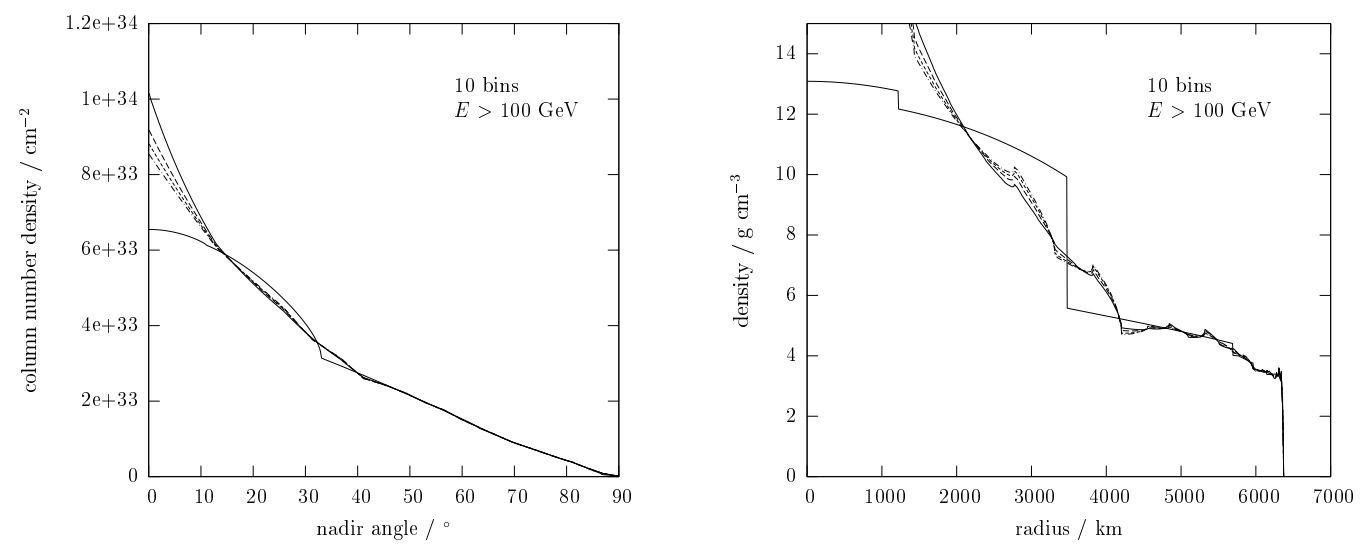

Figure 6.6: Left: The column number density reconstructed from an ideal muon event distribution without statistical fluctuations. 10 nadir angle bins are used, and an energy offset of $100 \mathrm{GeV}$ is assumed. The lines correspond to the upper (dashed) and lower MPR flux limit (dotted), Gen(0.5,1.5) (dot-dashed), and Gen(1.0,0.5) (solid line) as the initial neutrino spectrum. In addition, the column number density according to the PREM is shown (lower (at $0^{\circ}$ ) solid line). Right: The corresponding density distribution. The lines refer to the same initial spectra as in the left panel. The density distribution of the PREM is included as well (lower $\left(\right.$ at $\left.0^{\circ}\right)$ solid line).
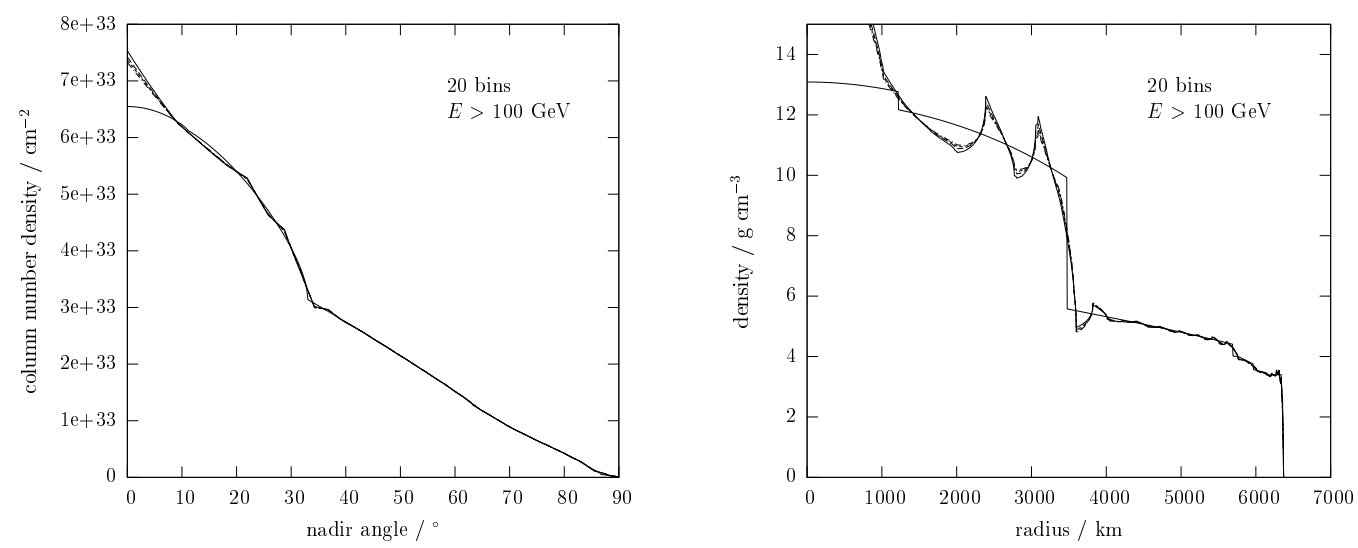

Figure 6.7: The same as Fig. 6.6, but for 20 nadir angle bins. 

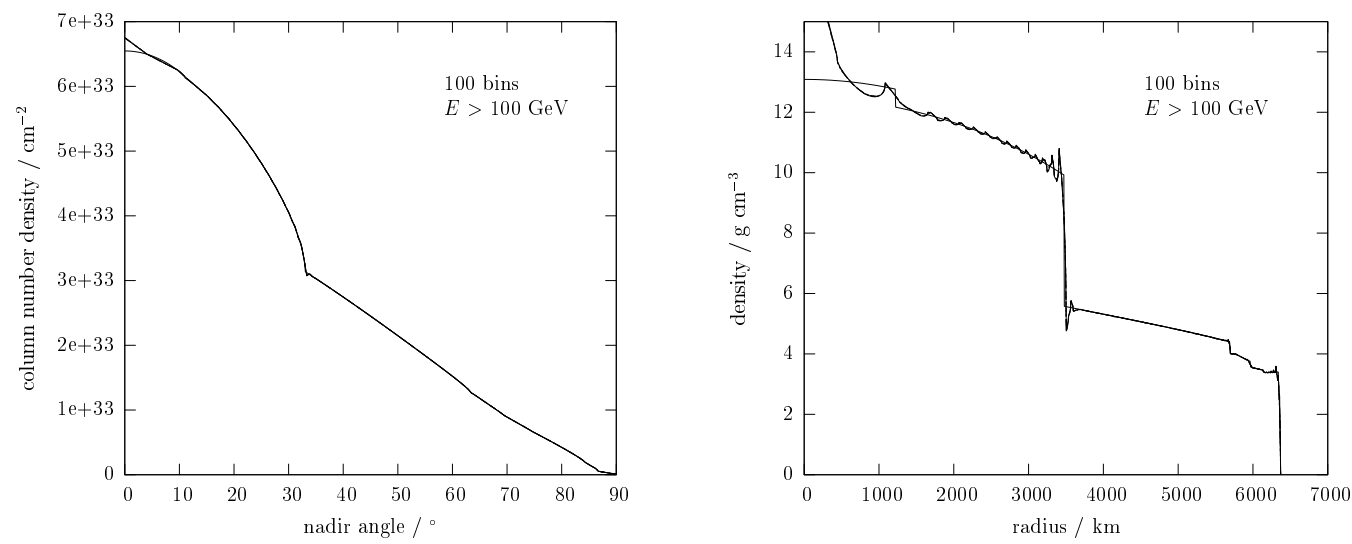

Figure 6.8: The same as Fig. 6.6, but for 100 nadir angle bins. As can be seen, the small inconsistency between the real and the approximated column number density curves between $0^{\circ}$ and $5^{\circ}$ completely changes the density distribution below a radius of $\approx 1000 \mathrm{~km}$.

Irrespective of the number of bins, we see that the results are (almost) independent of the specific initial neutrino spectrum, at least in the regime where they match the Preliminary Reference Earth Model. We accordingly restrict the analysis to one initial spectrum only. We choose the upper MPR flux limit, i.e. a power law with spectral index -2 .

In real life, detected numbers of events may be subject to considerable fluctuations. As stated before, we assume them to be Poisson deviates, the mean of which is equal to the ideal number of events in each bin, and we estimate the error inflicted by these fluctuations by the standard deviation obtained for 1000 simulated observations.

The quality of the density reconstruction depends on the overall number of events, the chosen number of nadir angle bins, and the energy offset assumed for the detector. Here, the overall number of events must be proportional to the uptime $t_{\mathrm{up}}$ of the detector, and we shall quote the results in terms of the latter.

Figs. 6.9 6.13 show the results of simulations for various combinations of the mentioned quantities. They clearly show that the quality of the reconstruction gets better for a longer uptime, which of course is a direct consequence of the fact that the ratio of the standard deviation of a binned event number $N_{i}$ and the event number itself scales as $\sqrt{N_{i}} / N_{i}$. In other words, the relative error in the number of events should be proportional to 
$t_{\mathrm{up}}^{-1 / 2}$ and hence should vanish in the limit of long observations.

On the other hand, increasing the energy offset has two consequences: Firstly, the overall number of events is diminished, which leads to a decreased accuracy. Secondly, moving the energy offset to higher values implies that some fraction of the (almost) nadir angle-independent low energy flux is disregarded. Hence propagation effects appear more prominent in the event distribution, which faciliates a reliable reconstruction of the column number density and thus increases the accuracy.

As can be seen from Figs. 6.11 and 6.12, for an increase of the energy offset from 100 to $1000 \mathrm{GeV}$, the two effects basically cancel. When moving to still higher energy offsets, the larger statistical error due to the diminished number of events starts to dominate, and for offsets above $10^{6} \mathrm{GeV}$ a tomography becomes completely impossible. Hence no improvement is attained from using an energy offset higher than $100 \mathrm{GeV}$, and we will continue to use this value for the rest of this section.

The "saw tooth structure" of the statistical errors in the plots mirrors a respective form of the reconstructed density. This in turn is ultimately due to the way we obtained the event rate, which for some given nadir angle bin tends to overestimate the rate in (roughly) one half of the bin, while underestimating it in the other half. Hence the column number density will be correspondingly under- and overestimated, and this leads to the mentioned saw tooth deviations seen in the density profile.

In general, we see that an optimistic neutrino source flux, a cubic kilometer detector, and several years of observation should yield a reasonable density profile for the mantle, and one might hope to see some evidence for the sharp density jump at the core-mantle boundary. The density profile inside the core, on the other hand, remains unresolved.

As we have seen, the latter has a profound reason apart from mere statistical fluctuations: Unless the available number of events allow a sufficiently fine binning for small nadir angles, the reconstruction of the density is severely limited by the arbitrariness of the choice for the event distribution consistent with the detected numbers of events.

Things could be improved by employing a more appropriate recipe for obtaining the continuous event distribution from the binned event numbers. However, one should not forget that in doing so one commits, at least in principle, a petitio principii, so that the amount of insight into the structure of the Earth would again be limited. We don't pursue such an analysis. 

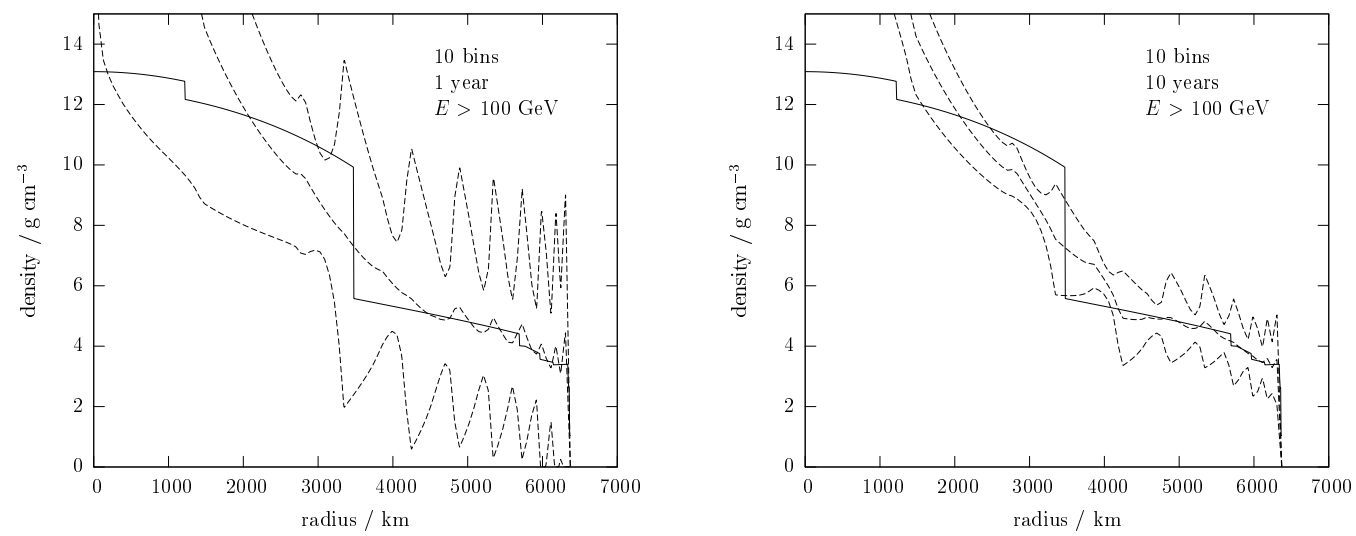

Figure 6.9: Left: The reconstructed density (middle) together with the expected observational uncertainties (bottom and top line), as obtained from performing a tomography by means of the events for the upper MPR flux limit. 10 nadir angle bins, an energy offset of $100 \mathrm{GeV}$, and a detector uptime of 1 year are assumed. In addition, the Prelimary Reference Earth Model (solid line) is shown. Right: The same as the left panel, but for an uptime of 10 years. See the main text for an explanation of the "saw tooths".
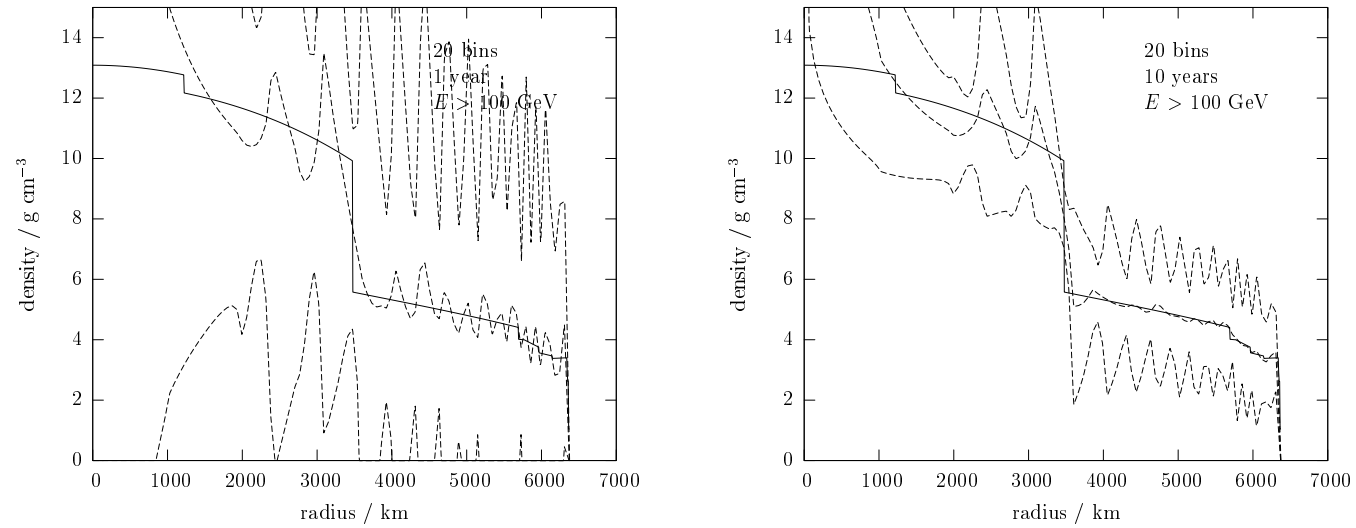

Figure 6.10: The same as Fig. 6.9, but for 20 nadir angle bins. Due to the immense statistical fluctuations, no reasonable statement concerning the density can be made for an uptime of 1 year. 

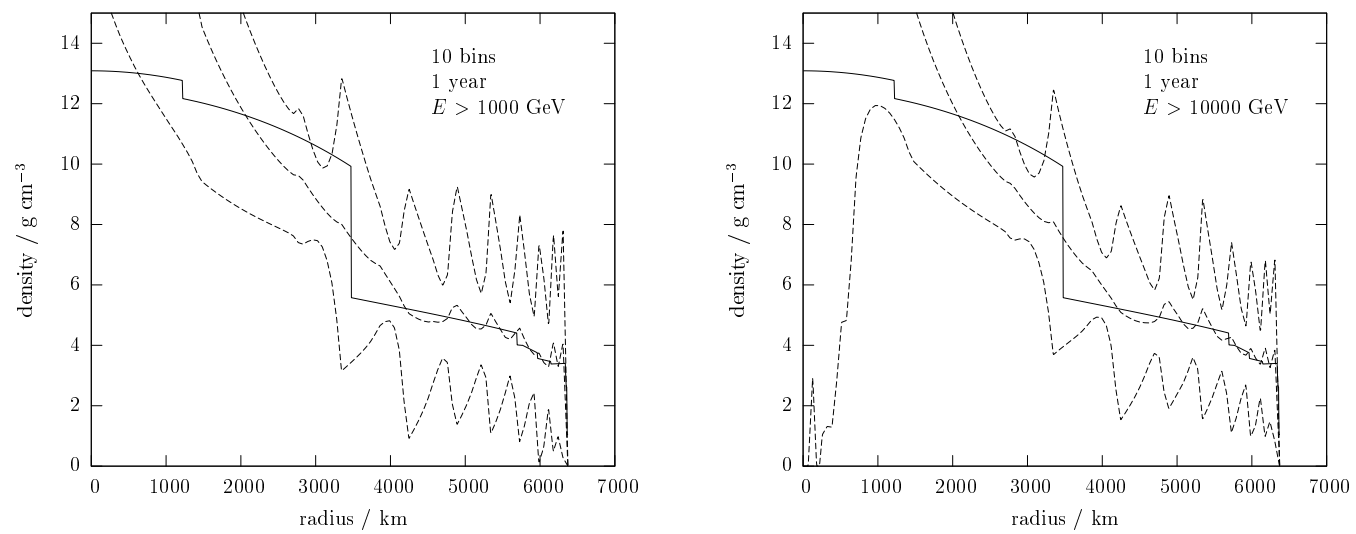

Figure 6.11: Left: The reconstructed density (middle) together with the expected observational uncertainties (bottom and top line), as obtained from performing a tomography by means of the events for the upper MPR flux limit. 10 nadir angle bins, an energy offset of $1000 \mathrm{GeV}$, and a detector uptime of 1 year are assumed. In addition, the Prelimary Reference Earth Model (solid line) is shown. Right: The same as the left panel, but for an energy offset of $10^{4} \mathrm{GeV}$.
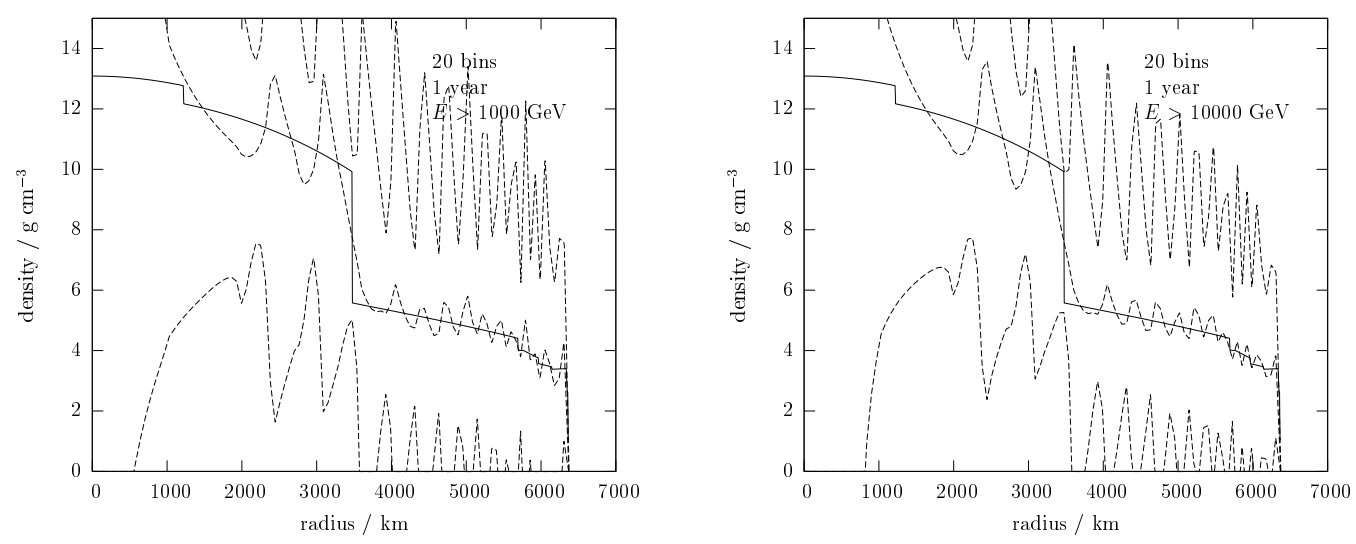

Figure 6.12: The same as the left panel of Fig. 6.10, but for an energy offset of $1000 \mathrm{GeV}$ (left) and $10^{4} \mathrm{GeV}$ (right panel). Again we note that no improvement in the accuracy is attained by choosing a higher energy cutoff. 

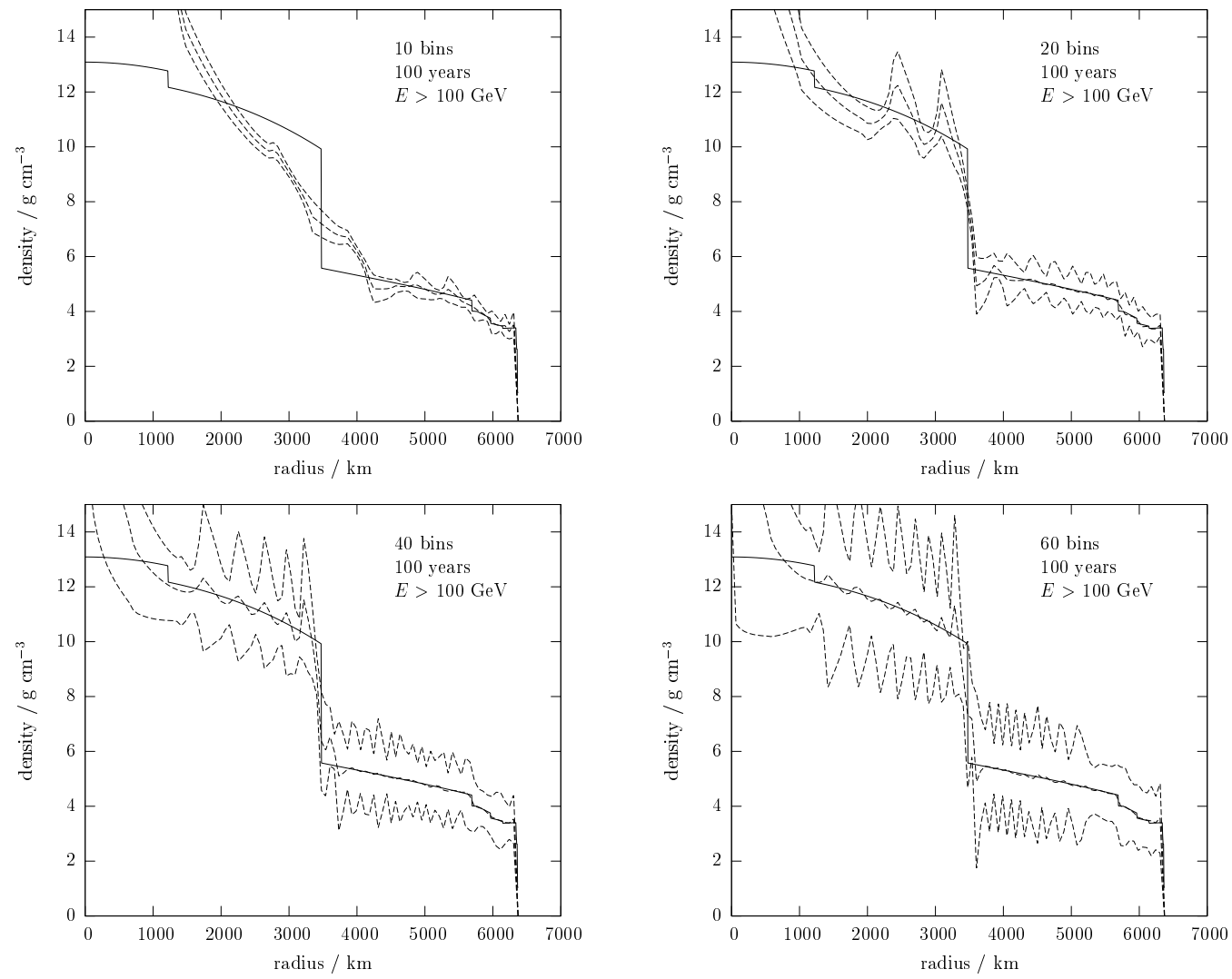

Figure 6.13: The reconstructed density distribution for 10 (upper left), 20 (upper right), 40 (lower left), and 60 nadir angle bins (lower right panel), if an uptime of 100 years and an energy offset of $100 \mathrm{GeV}$ are asumed. Obviously, an uptime of a hundred years is unrealistic, but an equivalent number of events might be obtained with techniques such as the detection of radio Cerenkov radiation. [112] 


\begin{tabular}{|c|c|c|c|}
\hline \multirow[t]{2}{*}{ region } & \multicolumn{3}{|c|}{ average density / $\mathrm{g} / \mathrm{cm}^{3}$} \\
\hline & $n=10$ & $n=20$ & $n=100$ \\
\hline \multicolumn{4}{|l|}{$t_{\mathrm{up}}=1 \mathrm{yr}$} \\
\hline inner core & $23.6 \pm 13.1$ & $17.4 \pm 16.8$ & $16.3 \pm 26.8$ \\
\hline outer core & $9.88 \pm 2.12$ & $10.8 \pm 2.98$ & $11.9 \pm 3.75$ \\
\hline mantle and crust & $4.68 \pm 0.505$ & $4.67 \pm 0.606$ & $5.45 \pm 0.723$ \\
\hline \multicolumn{4}{|l|}{$t_{\mathrm{up}}=10 \mathrm{yr}$} \\
\hline inner core & $22.1 \pm 3.89$ & $14.9 \pm 4.88$ & $12.9 \pm 7.49$ \\
\hline outer core & $9.92 \pm 0.682$ & $10.7 \pm 0.924$ & $11 \pm 1.07$ \\
\hline mantle and crust & $4.58 \pm 0.16$ & $4.48 \pm 0.196$ & $4.54 \pm 0.207$ \\
\hline \multicolumn{4}{|l|}{$t_{\mathrm{up}}=\infty$} \\
\hline inner core & $21.9 \pm 0$ & $14.7 \pm 0$ & $12.8 \pm 0$ \\
\hline outer core & $9.9 \pm 0$ & $10.7 \pm 0$ & $10.9 \pm 0$ \\
\hline mantle and crust & $4.58 \pm 0$ & $4.47 \pm 0$ & $4.46 \pm 0$ \\
\hline
\end{tabular}

Table 6.2: Average densities together with the expected observational uncertainties, as expected from performing a tomography by means of the events for the upper MPR flux limit. The values were obtained from 1000 simulated observations.

Instead we note that, as our continuous event distribution is consistent with the overall number of events, there must be regions where it over- and others where it underestimates the true distribution. This means, of course, that the same will be true for the reconstructed column number density and density. In other words, while the detailed reconstruction of the density might be pretty bad, there is hope that the average density

$$
\bar{\rho}\left(R_{1}, R_{2}\right)=\left(\frac{4}{3} \pi\left(R_{2}^{3}-R_{1}^{3}\right)\right)^{-1} \cdot 4 \pi \int_{R_{1}}^{R_{2}} r^{2} \rho(r) \mathrm{d} r
$$

of a sufficiently large shell with radii between $R_{1}$ and $R_{2}$ might be reconstructed more accurately. In order to check this, we compute the average density in the inner core (i.e. for radii less than $1221.5 \mathrm{~km}$ ), the outer core (i.e. for radii between 1221.5 and $3480 \mathrm{~km}$ ) and for the mantle (plus crust, i.e. for radii exceeding $3480 \mathrm{~km}$ ). The results for various numbers of nadir bins and detector uptimes are given in Table 6.2.

One sees easily that 10 years of observation would be required in order to get at least a rough estimate of the density in the inner core. On the other hand, the density in the outer core could be estimated with an inaccuracy of $20-30 \%$, and that in the mantle with an inaccuracy of $10-15 \%$ within 
one year. Ten years of observation would further diminish the inaccuracy to about $10 \%$ for the outer core and to about $5 \%$ for the mantle. Hence a tomography by means of high energy neutrinos could yield acceptable results for the average density in the outer core and mantle.

However, as these regions contribute the largest part to the overall mass of the Earth, it is reasonable to assume that a mass determination ought to be possible as well. While this would be of no immediate practical importance, it would constitute a confirmation of the value of this important quantity which does not depend on gravity.

Assuming the Preliminary Reference Earth Model and treating the Earth as a perfect sphere, the mass of the Earth can be computed by integrating the PREM density,

$$
\begin{aligned}
M_{\text {Earth,PREM }} & =\int_{0}^{R_{\text {Earth }}} \mathrm{d} r r^{2} \int_{4 \pi} \mathrm{d} \Omega \rho_{\text {PREM }}(r)=4 \pi \int_{0}^{R_{\text {Earth }}} r^{2} \rho_{\text {PREM }}(r) \mathrm{d} r \\
& =5.980 \times 10^{24} \mathrm{~kg},
\end{aligned}
$$

whereas the literature value is $M_{\text {Earth }}=5.9723(9) \times 10^{24} \mathrm{~kg}[70]$. In order to obtain an estimate from neutrino absorption, one just has to replace $\rho_{\text {PREM }}$ in Eq. 6.20 with the density obtained from the tomography described so far.

Intuitively, one might think that the value obtained for $M_{\text {Earth }}$ depends on the number $n$ of nadir angle bins, and that it might be pretty inaccurate for small $n$. While the first of these assertions is true, however, the second is correct only to some extent. Indeed, as can be seen from Table 6.3, even for $n=2$ the error of the result for an ideal detection lies below $5 \%$, and for $n=10$ it is already well below $1 \%$.

As usual, things get somewhat worse when taking statistical fluctuations into account. Fig. 6.14 shows the results for the upper MPR limit, asssuming an uptime of 1 and 10 years. We see that the true Earth mass lies well inside the range allowed by the statistical errors. Thus the Earth mass doesn't rule out our choice for the event rate, and using this mass as a constraint for the event rate is of rather limited value only. Accordingly, the accuracy claimed in [112], which employs such a constraint in order to obtain the density profile, should be taken with a large grain of salt.

Finally, it is instructive to ask what happens if the initial spectrum assumed for the calculation of the density from the event number distribution differs from the actual one. As an example, we have simulated events us- 


\begin{tabular}{|l|cccc|}
\hline source & \multicolumn{4}{|c|}{ Earth mass / kg } \\
& $\boldsymbol{n}=\mathbf{2}$ & $\boldsymbol{n}=\mathbf{1 0}$ & $\boldsymbol{n}=\mathbf{2 0}$ & $\boldsymbol{n}=\mathbf{3 0}$ \\
\hline upper MPR limit & $6.005 \times 10^{24}$ & $5.994 \times 10^{24}$ & $5.982 \times 10^{24}$ & $5.98 \times 10^{24}$ \\
lower MPR limit & $5.909 \times 10^{24}$ & $5.989 \times 10^{24}$ & $5.981 \times 10^{24}$ & $5.98 \times 10^{24}$ \\
Gen(0.5,1.0) & $5.954 \times 10^{24}$ & $5.993 \times 10^{24}$ & $5.982 \times 10^{24}$ & $5.98 \times 10^{24}$ \\
Gen(0.5,1.5) & $5.908 \times 10^{24}$ & $5.986 \times 10^{24}$ & $5.981 \times 10^{24}$ & $5.98 \times 10^{24}$ \\
Gen(1.0,0.5) & $6.289 \times 10^{24}$ & $6.009 \times 10^{24}$ & $5.983 \times 10^{24}$ & $5.98 \times 10^{24}$ \\
Gen(1.0,2.0) & $5.926 \times 10^{24}$ & $5.983 \times 10^{24}$ & $5.98 \times 10^{24}$ & $5.98 \times 10^{24}$ \\
\hline
\end{tabular}

Table 6.3: Values for the mass of the Earth obtained for various initial neutrino spectra, assuming ideal binned event rates in various numbers $n$ of nadir angle bins. The energy offset is taken to be $100 \mathrm{GeV}$.
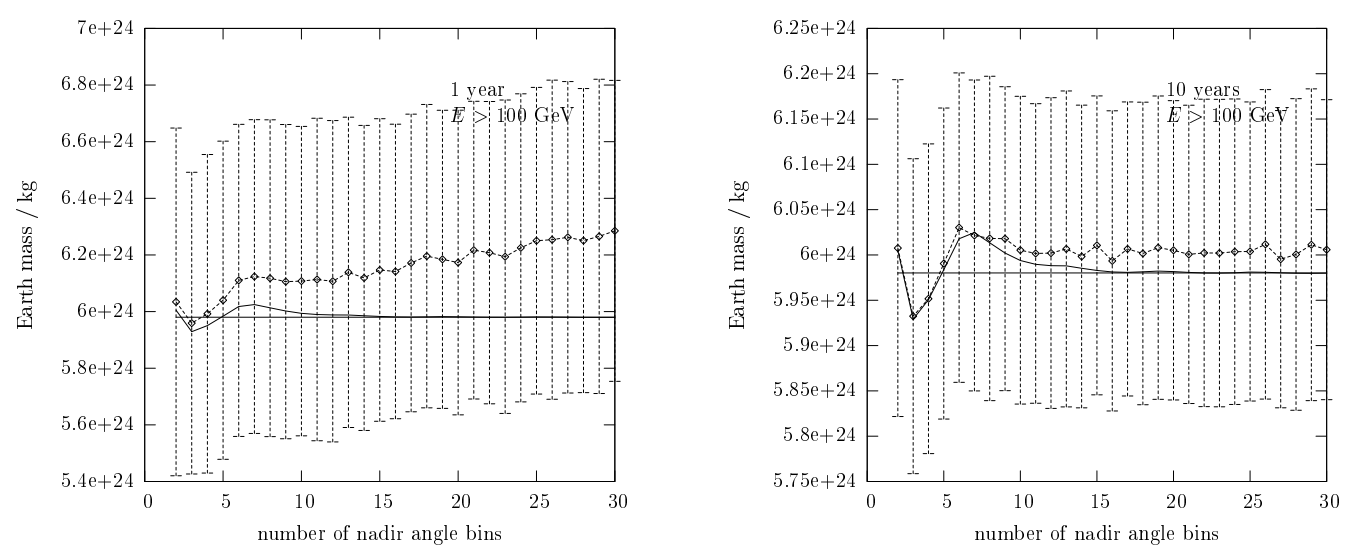

Figure 6.14: Left: Observational values for the mass $M_{\text {Earth }}$ of the Earth, as obtained from a tomography by means of the events expected for the upper MPR flux limit. 1000 observations have been simulated. $M_{\text {Earth }}$ is shown as a function of the number of nadir angle bins used (dashed line). The error bars refer to the uncertainty due to statistical fluctuations of the event rate. An energy offset of $100 \mathrm{GeV}$ and a detector uptime of 1 year are assumed. In addition, the result for an ideal detection with no statistical fluctuations (solid) and the value for the Preliminary Reference Earth Model (cf. Eq. 6.20) (horizontal line) are shown. Right: The same as the left panel, but for an uptime of 10 years. 

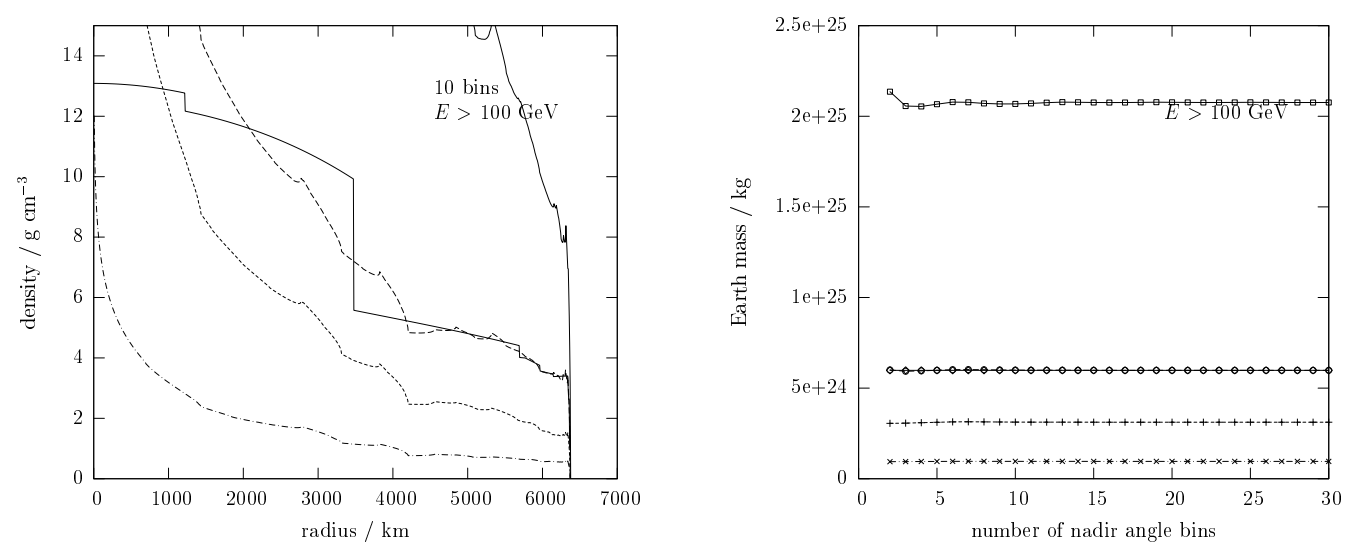

Figure 6.15: Left: The density distribution obtained for events from the upper (dashed) and lower MPR flux limit (dotted), Gen(0.5,1.5) (dot-dashed), and Gen(1.0,0.5) (solid line), if a power law with spectral index -2 is assumed for the initial neutrino spectrum when reconstructing the column number density. The density values are calculated for an ideal event rate without statistical fluctuations, 10 nadir angle bins, and an energy offset of $100 \mathrm{GeV}$. The PREM density distribution is shown as well (solid line). It is evident that for a successful tomography the knowledge of the initial spectral form is absolutely mandatory. Right: The corresponding mass of the Earth as a function of the number of nadir angle bins used. Again, (from top to bottom) Gen(1.0,0.5), the upper and lower MPR flux limit, and $\operatorname{Gen}(0.5,1.5)$ are shown for an energy offset of $100 \mathrm{GeV}$. As in the left panel, we see that previous knowledge of the initial form of the neutrino spectrum is required for any reasonable accuracy.

ing the lower MPR flux limit, $\operatorname{Gen}(0.5,1.5)$, and $\operatorname{Gen}(1.0,0.5)$ and performed the tomography under the assumption that the neutrinos were initially distributed according to a power law with spectral index -2 which results in the same overall event rate. The density distribution and Earth mass values thus obtained are shown in Fig. 6.15.

We clearly see that a faithful reconstruction of the terrestrial density distribution becomes utterly impossible, if we assume a wrong energy dependence for the initial neutrino spectrum. A back-of-the-envelope calculation may illustrate the reason. To this end, let us consider the upper MPR flux limit $A$ and the spectrum $B$ which is proportional to the lower MPR flux limit, but which is rescaled so that $\dot{N}_{A}\left(90^{\circ}\right)=\dot{N}_{B}\left(\theta=90^{\circ}\right)$.

Then at low energies the flux of $B$ must exceed that of $A$, and as lower 
energy neutrinos are less affected by the propagation through the Earth, the decrease in the event rate should be more prominent for $A$. Indeed, comparing the respective values in Table B.2, we note that the event rate at some $\theta<90^{\circ}$ relative to that at $90^{\circ}$ is diminshed to a larger extent for A than for B. Hence the reconstructed column number density values must be smaller for $B$, and the same has to hold for the density, as is borne out by Fig. 6.15 


\section{Chapter 7}

\section{Summary and outlook}

Neutrino astrophysics is on the edge of maturity, and it seems to be only a matter of time before the first neutrinos of astrophysical origin are detected in water-based Čerenkov detectors such as AMANDA or BAIKAL, and there is the promise that techniques such as radio or acoustic detection might further increase the efficiency of neutrino detection.

But one half of the neutrino flux has to cross the Earth prior to reaching the detector, and hence it is reasonable to ask how this affects the neutrino spectrum and thus event rates. These propagation effects can be described in terms of a set of coupled integro-differential equations, and we have investigated three methods for their solution.

Firstly, focussing on the tau neutrino propagation through a medium of constant density (such as a mountain), we showed that a combination of a Mellin and a Laplace transformation turns the set of equations for the tau neutrino and tauon into a set of difference equations for the transformed fluxes, the solution of which may be stated in terms of an infinite series. However, this approach requires a specific form for the neutrino-nucleon cross sections, and the series found is not particularly amenable to a numerical evaluation.

In case of instantaneous tauon decay the problem simplifies considerably, and there is no need for the assumption of a constant density or a particular cross section any longer. Then a Laplace transformation can be used to obtain an approximate solution in form of a finite series.

Secondly, a combined treatment of all neutrino flavors was accomplished by means of assuming a discrete set of energies, while retaining a continuous column number density. In this case the original set of equations simplifies 
into a set of ordinary differential equations, which can be solved analytically, yielding a finite series. Alas, this series involves small differences of large numbers, and for sufficiently small energy bins, the solution becomes numerically unstable. Therefore the possible number of energy bins is limited to some dozens of logarithmically spaced bins per factor 10 in the energy.

Finally, the $Z$ factor known from cascade theory allows to recast the equation describing the propagation of a single neutrino flavor into a form that can be solved approximately by means of iteration. We extended this formalism to the case of three neutrino flavors, and proved that there exists a simple measure for checking the accuracy of the solution. Hence we were able to show for a wide variety of initial neutrino spectra that the iterative ansatz yields solutions with a fully satisfactory accuracy.

In order to investigate the consequences of neutrino propagation for the event rates, we introduced a generic water-based Čerenkov detector model, which encompassed both contained and non-contained events, as well as radiative muon energy losses, taking into account that decaying tauons add to the electron and muon flux in the detector.

Calculating the event rates for various proposed astrophysical neutrino fluxes, we saw that propagation through the Earth leads to significant changes in the expected results. Indeed, considering the total event rate for energies above $100 \mathrm{GeV}$, the values for a nadir angle of $0^{\circ}$ and $90^{\circ}$ may differ by a factor from $\sim 2 / 3$ for power law spectra with an index of -2 to $\sim 100$ for spectra like that suggested for gamma-ray burst afterglows, which are considerably less steep. However, in practice, for most sources this angular dependence is masked by that due to the rock bed below the detector.

Contrary to the case of electron and muon neutrinos, the number of tau neutrinos is not diminished inside the Earth, and thus one might suppose that tau neutrinos will prove to dominate the detection rates. However, the calculations show this is not the case. Instead, there should be at least twice as many muon as tauon events, which can be explained by the fact that due to the rapid tauon decay, there are no non-contained tauon events. In addition, about one fifth of the tauon decays leads to the creation of a muon and hence adds further to the muon event rate.

Nonetheless tau neutrino propagation has a profound influence on the form of the $Z$ factors for electron and muon neutrinos, and hence a precise analysis of the neutrino propagation requires a combined treatment of all three neutrino flavors.

While the $Z$ factors and event rates were obtained for a variety of pro- 
posed sources, special emphasis was put on the Sun as a high-energy neutrino source. Because the respective neutrinos are created by cosmic ray impingement, which is a well-understood mechanism, it can be considered to be a guaranteed source and should lead to an event rate of several muon events per year in a $1 \mathrm{~km}^{3}$ detector. As neutrino flavor oscillations occur during the flight from Sun to Earth, tauon events are to be expected as well. Their detection and the ratio of muon and tauon events might constitute an independent (albeit rough) confirmation of the oscillation parameters deduced from other experiments.

Having seen that the neutrino flux varies significantly with the nadir angle, we turned to the question to what extent neutrino absorption and regeneration allow a tomography of the inner Earth. Performing this tomography requires the calculation of the inverse Radon transform of the column number density. To this end, we derived an analytic formula for the inverse transform of a piecewise polynomial function and applied it to the column number density reconstructed from (simulated) observations of an isotropic neutrino flux. We assumed that the energy dependence of the initial neutrino spectrum is known, but we required no knowledge concerning the energy dependence of the observed events.

The tomography faces a major obstacle. As realistic numbers of events are rather small, one has to use binned event rates, where the number of nadir angle bins can be of the order of 10 or 20 at most. This implies that the exact angular dependence of the event rate cannot be established, and one is forced to make some assumption regarding its form, which leads to a systematic error in addition to the statistical fluctuations of the event rate. All in all, in order to have a chance to see at least the core-mantle transition in a reconstructed density profile, one will need a total of several thousand events in 20 nadir angle bins.

Concerning averaged densities, the situation is ambivalent. While in the foreseeable future, high-energy neutrinos presumably won't allow any realistic measurement of the average density in the inner core, there is hope that their detection may confirm the value of the average densities in the outer core and mantle to within a few per cent.

Things are even better for the mass of the Earth, which is obtained by integrating the reconstructed density profile. Here, the inaccuracy turns out to be almost independent of the number of nadir angle bins and can be estimated to be about $1 \%$, if statistical fluctuations are ignored. A determination of the Earth mass by means of the formalism developed in this work 
seems possible. It would be completely independent of any measurement involving gravity.

The analysis of this work could be extended in various directions. For example, neutrino-electron scattering has not been taken into account. While its inclusion wouldn't change the overall picture, it would lead to significant changes for energies close to $6.3 \mathrm{PeV}$.

In addition, our analysis focussed on the propagation of neutrinos through the whole Earth and not, say, some mountain or the atmosphere, and therefore we could limit our calculations to the energy range below $10^{8} \mathrm{GeV}$. An extension to higher energies must take the radiative tauon energy losses and tauon-nucleon interactions into account, as their timescales become comparable to the tauon lifetime.

When discussing the tomography of the inner Earth, we were completely agnostic concerning the precise form of the expected neutrino event rate for some given neutrino flux, and we saw that this caused a significant systematic error. This error might be reduced by making additional assumptions concerning the event rate, such as that it mustn't be less than some minimum value in the middle of the Earth or that it doesn't contain any kinks. Care has to be exercised, though, as otherwise one might, for example, accidentally dismiss the core-mantle boundary as an artefact.

Furthermore, we saw that the density profile and Earth mass obtained from a neutrino event rate depend on the spectral form of the initial flux. Hence, demanding that the density and mass reconstructed from an event rate match the true ones might help to constrain the energy dependence of an observed neutrino flux. However, this would require a more thorough investigation of the consequences of uncertainties in the energy and angular dependence of a neutrino flux.

As can be seen from the example of the solar neutrino problem, neutrino astrophysics has a great potential of surprising astrophysicists and particle physicists alike. One may hypothesize, e.g., that neutrino oscillations on cosmological scales could turn out to be inconsistent with expectation. The neutrino speed might depend on energy. Or the neutrino-nucleon cross section might involve some non-standard model physics at ultra-high energies.

More likely than not, the observation of any such effect will require a detailed knowledge of the relation between the initial neutrino spectrum and the corresponding event rate, and thus a proper treatment of neutrino propagation, as carried out in this work, will be indispensable. 


\section{Appendix A}

\section{Software}

\section{A.1 TEMPEST}

For carrying out the neutrino propagation calculations in this work, the program "TEMPEST" was written. This section gives a brief introduction to its installation and usage.

\section{A.1.1 Requirements}

TEMPEST uses version 4.0 of Gnuplot [89] in order to produce its plots, so this program must be installed on the computer system used, and it has to be startable by means of the command gnuplot. As the relevant data is piped to a Gnuplot process duruing the execution of the program, both your $\mathrm{C}++$ compiler and your operating system must know about the popen() command, which should be declared in the cstdio.h header file.

In addition, TEMPEST employs version 3.2.3 of the cgicc library [53] as well as version 1.4 of the GNU Scientific Library [95]. Hence both libraries must be available on your computer, and the compiler must be able to find both their header and their object files.

If these requirements are met, any ANSI $\mathrm{C}++$ compliant compiler can be used to obtain a TEMPEST executable, as described in the next subsection.

\section{A.1.2 Installation}

Send an email to hettlage@astro.physik.uni-goettingen.de and ask for the file tempest.tar.gz. Move this file into a suitable directory. In the 
following, for the sake of definiteness we'll assume that this directory is /home/hettlage/programs/, but you may choose any name of your choice. Subsequently, unzip the file and extract its content, e.g. by means of

$$
\text { gunzip tempest.tar.gz; tar xvf tempest.tar; }
$$

Change into the newly created directory tempest and open the makefile Makefile with any text editor. You need to adapt this part of the makefile to your own needs:

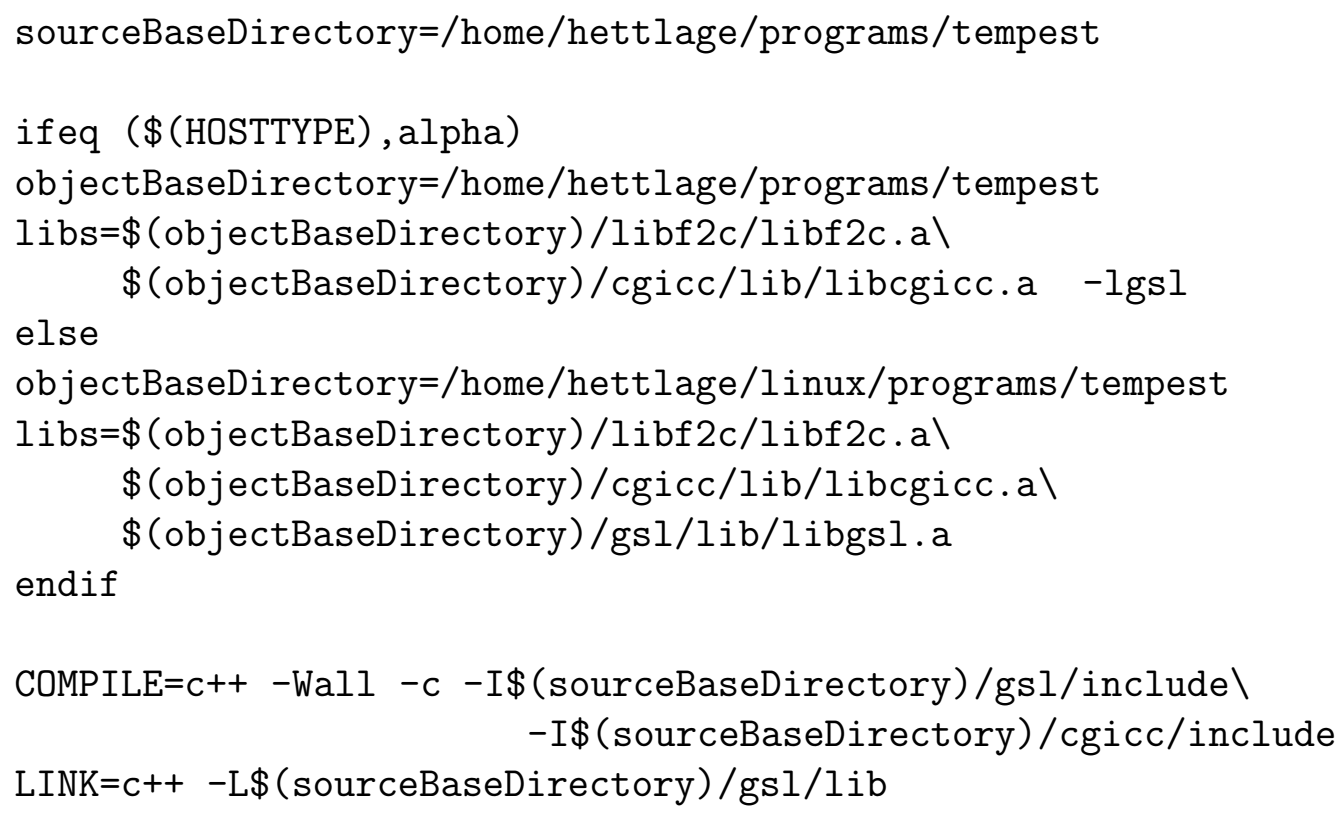

As this code illustrates, you may use the same source code for compiling and linking under two or more different operating systems. When adapting the code, you must ensure that all the libraries (and their header files) can be found by the compiler. If these were all installed in standard directories, you may leave out the lines concerning the objectBaseDirectory and libs variables, and you may set the values of the LINK and COMPILE variable to c++ and c++ -Wall -c, respectively.

Afterwards, run a make program that understands your makefile syntax. For the example shown above, GNU make would be a reasonable choice. If all goes well, just typing make or gmake might be sufficient.

If you encounter problems during compiling or linking TEMPEST, you should consult your local $\mathrm{C}++$ guru or send a mail with a detailed error description to hettlage@astro.physik.uni-goettingen.de. 


\section{A.1.3 Running the program}

TEMPEST is run from the command line, and is called using the following syntax:

$$
\text { run_tempest parameter_file }
$$

Note that you have to be in the TEMPEST directory (such as, for example, /home/hettlage/programs/tempest/) when starting the program. In the parameter file parameter_file, all the relevant parameters must be given in the form

\section{parameter name: parameter value}

with exactly one parameter per line. Their names and possible values are collected in Table A.1. Here, neutrino_type states whether neutrinos or antineutrinos are considered, energy_offset is the energy offset of the neutrino detector, number_of_nadir_bins denotes the number of bins used when binning the event rate, and number_of_iteration_steps gives the number of iterations used for computing the $Z$ factor. generic_cross_section_spread defines the value $s$ of Eq. 5.5.

The meaning of the secondary_parameter_values parameter depends on the kind of plot produced. It consists of a list of numbers, and for each list entry a curve is output. See below for an example.

All the other parameter names should be self-explanatory. A simple example of valid content for a parameter file thus would be:

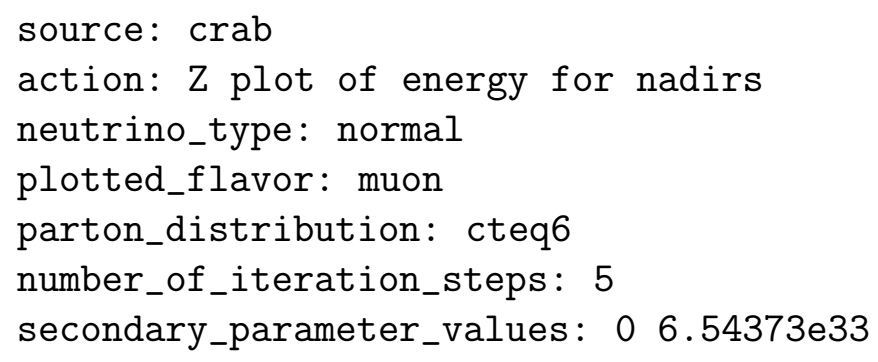

If run with this parameter file, TEMPEST would produce (on the screen) a plot of the $Z$ factor for muon neutrinos as a function of the neutrino energy for the column number densities $t=0$ and $t=6.54373 \times 10^{33} \mathrm{~g} / \mathrm{cm}^{2}$, assuming the Crab nebula as the source and using 5 iterations. 


\begin{tabular}{|l|l|}
\hline Parameter name & Possible values \\
\hline action & see Table A.2 \\
source & all source names \\
neutrino_type & normal, anti \\
parton_distribution & cteq6 \\
generic_cross_section_spread & all positive numbers \\
minimum_depth & all positive numbers \\
maximum_depth & all positive numbers \\
energy_offset & all positive numbers \\
number_of_nadir_bins & all positive integers \\
number_of_iteration_steps & all positive integers \\
plotted_flavors & a blank-separated list of electron, muon, tauon \\
minimum_abscissa_value & all real values \\
maximum_abscissa_value & all real values \\
minimum_ordinate_value & all real values \\
maximum_ordinate_value & all real values \\
secondary_parameter_values & a blank-separated list of positive numbers \\
format & postscript, ps, jpeg, jpg, png, gif \\
output & graph, source \\
output_file & a valid filename \\
\hline
\end{tabular}

Table A.1: Parameters used in a TEMPEST parameter file, together with their allowed values. See the main text for an explanation of the parameters. 


\begin{tabular}{|l|l|}
\hline Task & Value for action \\
\hline Plot $Z(E)$ for the given depths & Z plot of energy for depths \\
Plot $Z(E)$ for the given nadir angles & Z plot of energy for nadirs \\
Plot $\phi(E)$ for the given depths & flux plot of energy for depths \\
Plot $\phi(E)$ for the given nadir angles & flux plot of energy for nadirs \\
Plot $\phi(t)$ for the given energies & flux plot of depth \\
Plot $\phi(\theta)$ for the given energies & flux plot of nadir \\
Plot $S(E)$ for the given depths & shadowing factor plot of energy for depths \\
Plot $S(E)$ for the given nadir angles & shadowing factor plot of energy for nadirs \\
Plot $S(t)$ for the given energies & shadowing factor plot of depth \\
Plot $S(\theta)$ for the given energies & shadowing factor plot of nadir \\
Plot the $r(k)(E)$ for the given depths & Z accuracy plot of energy \\
Plot the $r^{(k)}(t)$ for the given energies & Z accuracy plot of depth \\
Plot $\phi_{\text {tot }}(t)$ & total flux plot of depth \\
Plot $\phi_{\text {tot }}(\theta)$ & total flux of nadir \\
Plot $\dot{N}_{(}(E)$ for the given depths & differential event rate plot of energy for depths \\
Plot $\dot{N}_{(}(E)$ for the given nadir angles & differential event rate plot of energy for nadirs \\
Plot $\dot{N}_{\text {tot }}\left(E_{\text {offset }}\right)$ for the given depths & total event rate plot of offset for depths \\
Plot $\dot{N}_{\text {tot }}\left(E_{\text {offset }}\right)$ for the given depths & total event rate plot of offset for nadir angles \\
Plot $\dot{N}_{\text {tot }}(t)$ for the given offset energies & total event rate plot of depth \\
Plot $\dot{N}_{\text {tot }}(\theta)$ for the given offset energies & total event rate plot of nadir \\
Plot the binned event rates & binned event rate plot \\
Do nothing & none \\
\hline
\end{tabular}

Table A.2: Possible tasks and the corresponding value of the action parameter. $S$ denotes the shadowing factor, $r^{(k)}$ the ratio of $Z$ for the $k$-th and the last iteration, $\phi$ and $\phi_{\text {tot }}$ the differential and total neutrino flux, $\dot{N}$ and $\dot{N}_{\text {tot }}$ the differential and total event rate, $E$ the neutrino energy, $E_{\text {offset }}$ the energy offset for the neutrino detector, $t$ the column number density ("depth"), and $\theta$ the nadir angle. 


\section{A.1.4 Adding a new source}

When adding a new source to TEMPEST, you first have to create the file containing the neutrino fluxes prior to crossing the Earth. Each line must start with a neutrino energy (in $\mathrm{GeV}$ ), followed by the corresponding fluxes of $\nu_{e}, \nu_{\mu}, \nu_{\tau}, \bar{\nu}_{e}, \bar{\nu}_{\mu}$ and $\bar{\nu}_{\tau}$, and the energies must appear in ascending order.

After that, you have to create a directory for the new source in /home/hettlage/programs/tempest/sources/, and you have to copy the file containing the fluxes to that directory. The names of the directory and the flux file must be the same. Indeed, it is precisely this name that must be used as the value for the source parameter.

If you run TEMPEST with a parameter file for the new source, all the necessary computations for obtaining the required files will be carried out automatically. Be warned though: Depending on the computer used, this may take several days! 


\section{Appendix B}

\section{Event rates and $Z$ factor inaccuracies}

\section{B.1 Astrophysical neutrino event rates}

In Chapter 5, the total muon event rate is plotted as a function of the nadir angle $\theta$ and the detector energy offset $E_{\text {offset }}$ for a wide variety of astrophysical sources. For convenience, the respective rates at $\theta=0^{\circ}, 30^{\circ}, 60^{\circ}$, and $90^{\circ}$ are listed for $E_{\text {offset }}=100 \mathrm{GeV}$ in Table B.1 and for $E_{\text {offset }}=1000 \mathrm{GeV}$ in Table B.3. Tables B.5 and B.7 contain the corresponding tauon event rates. When looking at these values, one shouldn't forget about the caveat stated with regard to Figs. 5.1 and 5.2 .

Some care has to be taken concerning the units in the tables. In case of a diffuse flux, the total event rate is to be understood as a number per solid angle and time, whereas in case of a point source, it just constitutes a number per time.

As an isotropic flux by definition is the same from all directions, the event rate $N_{l, 2 \pi}$ of flavor $l$ due to all upward moving neutrinos from an isotropic diffuse source is simply given by the integral

$$
\dot{N}_{l, 2 \pi}=2 \pi \int_{0}^{\pi / 2} \mathrm{~d} \theta \sin \theta \dot{N}_{l}(\theta) .
$$

Obviously, no comparable quantitity exists for a point source. If we assume that the sensitivity of our detector is the same for downward moving neutrinos and for neutrinos moving horizontally, the overall event rate $N_{l, 4 \pi}$ due to 
neutrinos from any direction has the form

$$
\dot{N}_{l, 4 \pi}=\dot{N}_{l, 2 \pi}+2 \pi \dot{N}(\theta=\pi / 2) .
$$

This is an overestimate of course, as the amount of water, ice, or rock is limited above the detector. For the considered isotropic neutrino fluxes, the values of $\dot{N}_{l, 2 \pi}$ are included in the tables.

As the event rates obtained in Chapter 5 are computed for the generic detector introduced in Sect. 4.2, they involve the rock correction factor $R$ defined in Eq. 4.6,

$$
R(\theta)=\left\{\begin{array}{ll}
0.70+0.48 \cos \theta & \left(\theta \leqslant 95^{\circ}\right) \\
0.70+0.48 \cos \left(95^{\circ}\right) & \left(\theta>95^{\circ}\right)
\end{array},\right.
$$

which tends to mask the dependence on the column number density crossed by the neutrinos. For this reason, the muon event rates without this factor are given in Tables B.2 and B.4, and the corresponding tauon event rates are provided in Tables B.6 and B.8. These event rates have been renormalized so that they are equal to the corresponding event rates with the correction factor for a nadir angle of $90^{\circ}$ (cf. the discussion at the end of Sect. 4.2).

\section{B.2 Inaccuracy of the $Z$ factor}

In Sect. 3.4, it has been shown that some $Z$ factor can be considered to be accurate at some energy $E$ and nadir angle $\theta$, if the condition

$$
\Delta Z(E, \theta) / Z(E, \theta) \ll 1
$$

is fulfilled, where $\Delta Z$ is given by Eq. 3.51. Hence the quantity $(\Delta Z / Z)_{\max }$ defined as

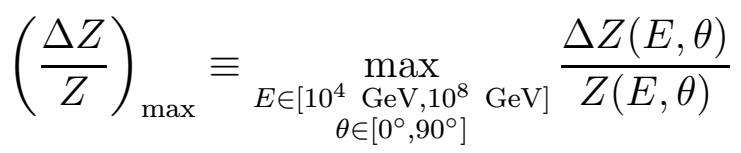

constitutes a measure for the accuracy of the $Z$ factor. While the choice for the range of nadir angles is obvious in this definition, the energy range might deserve a brief explanation. The upper limit $10^{8} \mathrm{GeV}$ is motivated by the fact that at higher energies, the flux practically vanishes due to absorption. Conversely, for energies less than $10^{4} \mathrm{GeV}$, absorption can be neglected and the $Z$ factor has no real significance. In Table B.9, the value of $(\Delta Z / Z)_{\max }$ is provided for the astrophysical sources and flux limits discussed in Chapter 5 . 


\begin{tabular}{|c|c|c|c|c|c|}
\hline \multirow[t]{2}{*}{ source } & \multicolumn{4}{|c|}{$\dot{N}_{\mu}(\theta)\left(\mathrm{yr}^{-1}\left(\mathrm{sr}^{-1}\right)\right)$} & \multirow[t]{2}{*}{$\dot{N}_{\mu, 2 \pi}\left(\mathrm{yr}^{-1}\right)$} \\
\hline & $\theta=0^{\circ}$ & $\theta=30^{\circ}$ & $\theta=60^{\circ}$ & $\theta=90^{\circ}$ & \\
\hline RX J1713.7-3946 & 7.5 & 7.7 & 7.2 & 5.9 & - \\
\hline Crab & 1.8 & 1.8 & 1.6 & 1.3 & - \\
\hline shocked pulsar wind & 1.5 & 1.7 & 1.9 & 1.7 & - \\
\hline SS433 & 930 & 970 & 950 & 850 & 一 \\
\hline SGR 1806-20 & 670 & 720 & 730 & 660 & - \\
\hline Cyg OB2 & 12 & 11 & 8.7 & 6.3 & - \\
\hline $\begin{array}{l}\text { Galactic center } \\
\text { (HESS, EHECR) }\end{array}$ & 4.4 & 4.4 & 4 & 3.2 & - \\
\hline Galactic plane & 0.28 & 0.27 & 0.23 & 0.17 & - \\
\hline $\begin{array}{ll}\text { Galactic } & \text { center } \\
\text { (Candia) } & \end{array}$ & 1200 & 1200 & 1000 & 800 & - \\
\hline $\begin{array}{l}\text { Galactic latitude } 45^{\circ} \\
\text { (Candia) }\end{array}$ & 300 & 290 & 250 & 190 & - \\
\hline GBBs (De Paolis) & 1.9 & 2 & 1.9 & 1.6 & 12 \\
\hline GRB941017 & 0.0031 & 0.0054 & 0.011 & 0.02 & 一 \\
\hline $\begin{array}{l}\text { GRB flux limit } \\
(\mathrm{MPR})\end{array}$ & 2300 & 2400 & 2300 & 1900 & $1.4 \times 10^{4}$ \\
\hline $\begin{array}{l}\text { GRB flux limit } \\
\text { (WB) }\end{array}$ & 0.28 & 0.37 & 0.5 & 0.58 & 3.1 \\
\hline GRB afterglow & 2.7 & 4.6 & 11 & 130 & 140 \\
\hline AGN jet & $2.2 \times 10^{-5}$ & $3.5 \times 10^{-5}$ & $7.9 \times 10^{-5}$ & 0.00046 & 0.00076 \\
\hline $3 \mathrm{C} 273(p p)$ & 13 & 13 & 13 & 11 & - \\
\hline $3 \mathrm{C} 273(p \gamma)$ & 0.069 & 0.11 & 0.23 & 0.49 & - \\
\hline radio quiet $\mathrm{AGN}$ & 0.89 & 1.1 & 1.5 & 1.6 & - \\
\hline $\begin{array}{l}\text { FR-II radio galaxies } \\
\text { and blazars }\end{array}$ & 160 & 170 & 170 & 150 & 1000 \\
\hline $1 \mathrm{ES} 1959+650$ & 3.9 & 4.2 & 4.2 & 3.7 & - \\
\hline $\begin{array}{l}\text { black hole forma- } \\
\text { tion (cluster formed } \\
\text { early) }\end{array}$ & 40 & 42 & 42 & 37 & - \\
\hline $\begin{array}{l}\text { black hole formation } \\
\text { (cluster formed late) }\end{array}$ & 350 & 380 & 380 & 350 & - \\
\hline galaxy clusters & 1.7 & 1.8 & 1.8 & 1.6 & 11 \\
\hline upper MPR limit & 1100 & 1100 & 1100 & 980 & 6900 \\
\hline lower MPR limit & 800 & 850 & 830 & 690 & 5100 \\
\hline WB limit & 29 & 30 & 30 & 27 & 190 \\
\hline AMANDA limit & 410 & 430 & 430 & 380 & 2600 \\
\hline
\end{tabular}

Table B.1: Total muon event rates $\dot{N}_{\mu}$ at various nadir angles $\theta$ for the astrophysical sources discussed in Chapter 5. A detector energy offset of $100 \mathrm{GeV}$ is assumed. In case of the isotropic source spectra, the integral $\dot{N}_{\mu, 2 \pi}$ of the total event rate over all nadir angles from $0^{\circ}$ to $90^{\circ}$ is given as well. 
160 APPENDIX B. EVENT RATES AND Z FACTOR INACCURACIES

\begin{tabular}{|c|c|c|c|c|c|}
\hline \multirow[t]{2}{*}{ source } & \multicolumn{4}{|c|}{$\dot{N}_{\mu}(\theta)\left(\mathrm{yr}^{-1}\left(\mathrm{sr}^{-1}\right)\right)[$ no rock correction] } & \multirow[t]{2}{*}{$\dot{N}_{\mu, 2 \pi}\left(\mathrm{yr}^{-1}\right)$} \\
\hline & $\theta=0^{\circ}$ & $\theta=30^{\circ}$ & $\theta=60^{\circ}$ & $\theta=90^{\circ}$ & \\
\hline RX J1713.7-3946 & 4.5 & 4.8 & 5.4 & 5.9 & - \\
\hline Crab & 1.1 & 1.1 & 1.2 & 1.3 & - \\
\hline shocked pulsar wind & 0.9 & 1.1 & 1.4 & 1.7 & - \\
\hline SS433 & 550 & 610 & 710 & 850 & - \\
\hline SGR 1806-20 & 400 & 450 & 540 & 660 & - \\
\hline Cyg OB2 & 6.9 & 6.7 & 6.5 & 6.3 & - \\
\hline $\begin{array}{l}\text { Galactic center } \\
\text { (HESS, EHECR) }\end{array}$ & 2.6 & 2.7 & 3 & 3.2 & - \\
\hline Galactic plane & 0.17 & 0.17 & 0.17 & 0.17 & - \\
\hline $\begin{array}{l}\text { Galactic } \quad \text { center } \\
\text { (Candia) }\end{array}$ & 720 & 740 & 780 & 800 & - \\
\hline $\begin{array}{l}\text { Galactic latitude } 45^{\circ} \\
\text { (Candia) }\end{array}$ & 180 & 180 & 190 & 190 & - \\
\hline GBBs (De Paolis) & 1.1 & 1.3 & 1.4 & 1.6 & 8.9 \\
\hline GRB941017 & 0.0019 & 0.0034 & 0.0084 & 0.02 & - \\
\hline $\begin{array}{l}\text { GRB flux limit } \\
\text { (MPR) }\end{array}$ & 1300 & 1500 & 1700 & 1900 & $1.1 \times 10^{4}$ \\
\hline $\begin{array}{lll}\text { GRB } & \text { flux } & \text { limit } \\
\text { (WB) } & & \end{array}$ & 0.16 & 0.23 & 0.38 & 0.58 & 2.4 \\
\hline GRB afterglow & 1.6 & 2.9 & 8.4 & 130 & 130 \\
\hline AGN jet & $1.3 \times 10^{-5}$ & $2.2 \times 10^{-5}$ & $5.9 \times 10^{-5}$ & 0.00046 & 0.00065 \\
\hline $3 \mathrm{C} 273(p p)$ & 7.4 & 8.5 & 10 & 11 & - \\
\hline 3C273 $(p \gamma)$ & 0.041 & 0.072 & 0.17 & 0.49 & - \\
\hline radio quiet AGN & 0.53 & 0.72 & 1.1 & 1.6 & - \\
\hline $\begin{array}{l}\text { FR-II radio galaxies } \\
\text { and blazars }\end{array}$ & 94 & 110 & 120 & 150 & 780 \\
\hline 1ES $1959+650$ & 2.3 & 2.6 & 3.1 & 3.7 & - \\
\hline $\begin{array}{l}\text { black hole forma- } \\
\text { tion (cluster formed } \\
\text { early) }\end{array}$ & 24 & 27 & 31 & 37 & - \\
\hline $\begin{array}{l}\text { black hole formation } \\
\text { (cluster formed late) }\end{array}$ & 210 & 240 & 290 & 350 & - \\
\hline galaxy clusters & 1 & 1.2 & 1.4 & 1.6 & 8.5 \\
\hline upper MPR limit & 630 & 700 & 830 & 980 & 5200 \\
\hline lower MPR limit & 470 & 530 & 620 & 690 & 3800 \\
\hline WB limit & 17 & 19 & 23 & 27 & 140 \\
\hline AMANDA limit & 240 & 270 & 320 & 380 & 2000 \\
\hline
\end{tabular}

Table B.2: Total muon event rates $\dot{N}_{\mu}$ at various nadir angles $\theta$ for the astrophysical sources discussed in Chapter 5, if the rock correction factor (Eq. B.1) is ignored. The vales are normalized, so that they agree with those of Table B.1 at $\theta=90^{\circ}$. A detector energy offset of $100 \mathrm{GeV}$ is assumed. In case of the isotropic source spectra, the integral $\dot{N}_{\mu, 2 \pi}$ of the total event rate over all nadir angles from $0^{\circ}$ to $90^{\circ}$ is given as well. Fluences have been divided by $1 \mathrm{yr}$. 


\begin{tabular}{|c|c|c|c|c|c|}
\hline \multirow[t]{2}{*}{ source } & \multicolumn{4}{|c|}{$\dot{N}_{\mu}(\theta)\left(\mathrm{yr}^{-1}\left(\mathrm{sr}^{-1}\right)\right)$} & \multirow[t]{2}{*}{$\dot{N}_{\mu, 2 \pi}\left(\mathrm{yr}^{-1}\right)$} \\
\hline & $\theta=0^{\circ}$ & $\theta=30^{\circ}$ & $\theta=60^{\circ}$ & $\theta=90^{\circ}$ & \\
\hline RX J1713.7-3946 & 3.1 & 3.4 & 3.5 & 3 & - \\
\hline Crab & 0.88 & 0.92 & 0.87 & 0.69 & - \\
\hline shocked pulsar wind & 1.2 & 1.4 & 1.6 & 1.4 & - \\
\hline SS433 & 560 & 640 & 690 & 660 & - \\
\hline SGR 1806-20 & 370 & 430 & 470 & 470 & - \\
\hline Cyg OB2 & 0.8 & 0.79 & 0.71 & 0.54 & - \\
\hline $\begin{array}{l}\text { Galactic center } \\
\text { (HESS, EHECR) }\end{array}$ & 1.1 & 1.3 & 1.3 & 1.2 & - \\
\hline Galactic plane & 0.0081 & 0.008 & 0.0071 & 0.0055 & - \\
\hline $\begin{array}{l}\text { Galactic center } \\
\text { (Candia) }\end{array}$ & 280 & 300 & 280 & 230 & - \\
\hline $\begin{array}{l}\text { Galactic latitude } 45^{\circ} \\
\text { (Candia) }\end{array}$ & 58 & 60 & 56 & 44 & 一 \\
\hline GBBs (De Paolis) & 0.91 & 1 & 1.1 & 0.95 & 6.5 \\
\hline GRB941017 & 0.0029 & 0.0051 & 0.011 & 0.02 & - \\
\hline $\begin{array}{l}\text { GRB flux limit } \\
(\mathrm{MPR})\end{array}$ & 1100 & 1300 & 1300 & 1200 & 8100 \\
\hline $\begin{array}{l}\text { GRB flux limit } \\
\text { (WB) }\end{array}$ & 0.24 & 0.33 & 0.47 & 0.55 & 2.8 \\
\hline GRB afterglow & 2.5 & 4.3 & 11 & 130 & 140 \\
\hline AGN jet & $1.9 \times 10^{-5}$ & $3.3 \times 10^{-5}$ & $7.7 \times 10^{-5}$ & 0.00046 & 0.00075 \\
\hline $3 \mathrm{C} 273(p p)$ & 7.3 & 8.4 & 9 & 7.9 & - \\
\hline $3 \mathrm{C} 273(p \gamma)$ & 0.064 & 0.11 & 0.23 & 0.49 & - \\
\hline radio quiet $\mathrm{AGN}$ & 0.74 & 0.99 & 1.3 & 1.5 & - \\
\hline $\begin{array}{l}\text { FR-II radio galaxies } \\
\text { and blazars }\end{array}$ & 83 & 95 & 100 & 98 & 630 \\
\hline 1ES $1959+650$ & 2.1 & 2.4 & 2.6 & 2.5 & 一 \\
\hline $\begin{array}{l}\text { black hole forma- } \\
\text { tion (cluster formed } \\
\text { early) }\end{array}$ & 21 & 24 & 26 & 25 & - \\
\hline $\begin{array}{l}\text { black hole formation } \\
\text { (cluster formed late) }\end{array}$ & 200 & 230 & 260 & 260 & 一 \\
\hline galaxy clusters & 0.92 & 1 & 1.1 & 1 & 6.8 \\
\hline upper MPR limit & 550 & 630 & 690 & 660 & 4200 \\
\hline lower MPR limit & 460 & 520 & 540 & 470 & 3300 \\
\hline WB limit & 15 & 17 & 19 & 18 & 110 \\
\hline AMANDA limit & 210 & 240 & 260 & 250 & 1600 \\
\hline
\end{tabular}

Table B.3: Total muon event rates $\dot{N}_{\mu}$ at various nadir angles $\theta$ for the astrophysical sources discussed in Chapter 5. A detector energy offset of $1000 \mathrm{GeV}$ is assumed. In case of the isotropic source spectra, the integral $\dot{N}_{\mu, 2 \pi}$ of the total event rate over all nadir angles from $0^{\circ}$ to $90^{\circ}$ is given as well. Fluences have been divided by $1 \mathrm{yr}$. 
162 APPENDIX B. EVENT RATES AND Z FACTOR INACCURACIES

\begin{tabular}{|c|c|c|c|c|c|}
\hline source & $\begin{array}{c}\dot{N}_{\mu}(\theta)(\mathrm{yr} \\
\theta=0^{\circ}\end{array}$ & $\begin{array}{l}\left.-1\left(\mathrm{sr}^{-1}\right)\right) \\
\theta=30^{\circ}\end{array}$ & $\begin{array}{c}\text { [no rock cc } \\
\theta=60^{\circ}\end{array}$ & $\begin{array}{r}\text { rrection] } \\
\theta=90^{\circ}\end{array}$ & $\dot{N}_{\mu, 2 \pi}\left(\mathrm{yr}^{-1}\right)$ \\
\hline RX J1713.7-3946 & 1.8 & 2.1 & 2.6 & 3 & - \\
\hline Crab & 0.52 & 0.58 & 0.65 & 0.69 & - \\
\hline shocked pulsar wind & 0.7 & 0.88 & 1.2 & 1.4 & - \\
\hline SS433 & 330 & 400 & 510 & 660 & - \\
\hline SGR 1806-20 & 220 & 270 & 350 & 470 & - \\
\hline Cyg OB2 & 0.47 & 0.5 & 0.53 & 0.54 & - \\
\hline $\begin{array}{l}\text { Galactic center } \\
\text { (HESS, EHECR) }\end{array}$ & 0.66 & 0.79 & 1 & 1.2 & - \\
\hline Galactic plane & 0.0048 & 0.005 & 0.0053 & 0.0055 & - \\
\hline $\begin{array}{l}\text { Galactic } \quad \text { center } \\
\text { (Candia) }\end{array}$ & 170 & 190 & 210 & 230 & - \\
\hline $\begin{array}{l}\text { Galactic latitude } 45^{\circ} \\
\text { (Candia) }\end{array}$ & 34 & 37 & 41 & 44 & - \\
\hline GBBs (De Paolis) & 0.54 & 0.64 & 0.8 & 0.95 & 4.9 \\
\hline GRB941017 & 0.0017 & 0.0032 & 0.0082 & 0.02 & - \\
\hline $\begin{array}{l}\text { GRB flux limit } \\
\text { (MPR) }\end{array}$ & 670 & 800 & 990 & 1200 & 6100 \\
\hline $\begin{array}{l}\text { GRB flux limit } \\
\text { (WB) }\end{array}$ & 0.14 & 0.21 & 0.35 & 0.55 & 2.2 \\
\hline GRB afterglow & 1.5 & 2.7 & 8.3 & 130 & 120 \\
\hline AGN jet & $1.2 \times 10^{-5}$ & $2 \times 10^{-5}$ & $5.7 \times 10^{-5}$ & 0.00046 & 0.00064 \\
\hline $3 \mathrm{C} 273(p p)$ & 4.3 & 5.3 & 6.7 & 7.9 & - \\
\hline $3 \mathrm{C} 273(p \gamma)$ & 0.038 & 0.069 & 0.17 & 0.49 & - \\
\hline radio quiet AGN & 0.44 & 0.62 & 1 & 1.5 & - \\
\hline $\begin{array}{l}\text { FR-II radio galaxies } \\
\text { and blazars }\end{array}$ & 49 & 59 & 77 & 98 & 480 \\
\hline 1ES $1959+650$ & 1.2 & 1.5 & 1.9 & 2.5 & - \\
\hline $\begin{array}{l}\text { black hole forma- } \\
\text { tion (cluster formed } \\
\text { early) }\end{array}$ & 12 & 15 & 19 & 25 & - \\
\hline $\begin{array}{l}\text { black hole formation } \\
\text { (cluster formed late) }\end{array}$ & 120 & 140 & 190 & 260 & - \\
\hline galaxy clusters & 0.54 & 0.65 & 0.83 & 1 & 5.2 \\
\hline upper MPR limit & 330 & 400 & 510 & 660 & 3200 \\
\hline lower MPR limit & 270 & 320 & 400 & 470 & 2500 \\
\hline WB limit & 8.9 & 11 & 14 & 18 & 87 \\
\hline AMANDA limit & 130 & 150 & 200 & 250 & 1200 \\
\hline
\end{tabular}

Table B.4: Total muon event rates $\dot{N}_{\mu}$ at various nadir angles $\theta$ for the astrophysical sources discussed in Chapter 5, if the rock correction factor (Eq. B.1) is ignored. The vales are normalized, so that they agree with those of Table B.3 at $\theta=90^{\circ}$. A detector energy offset of $1000 \mathrm{GeV}$ is assumed. In case of the isotropic source spectra, the integral $\dot{N}_{\mu, 2 \pi}$ of the total event rate over all nadir angles from $0^{\circ}$ to $90^{\circ}$ is given as well. Fluences have been divided by $1 \mathrm{yr}$. 


\begin{tabular}{|c|c|c|c|c|c|}
\hline \multirow[t]{2}{*}{ source } & \multicolumn{4}{|c|}{$\dot{N}_{\tau}(\theta)\left(\mathrm{yr}^{-1}\left(\mathrm{sr}^{-1}\right)\right)$} & \multirow[t]{2}{*}{$\dot{N}_{\tau, 2 \pi}\left(\mathrm{yr}^{-1}\right)$} \\
\hline & $\theta=0^{\circ}$ & $\theta=30^{\circ}$ & $\theta=60^{\circ}$ & $\theta=90^{\circ}$ & \\
\hline RX J1713.7-3946 & 3.3 & 3.3 & 2.9 & 2.3 & - \\
\hline Crab & 0.68 & 0.67 & 0.59 & 0.45 & - \\
\hline shocked pulsar wind & 0.46 & 0.5 & 0.5 & 0.43 & - \\
\hline $\mathrm{SS} 433$ & 270 & 280 & 270 & 230 & - \\
\hline SGR 1806-20 & 250 & 260 & 250 & 210 & - \\
\hline Cyg OB2 & 6.5 & 5.9 & 4.9 & 3.5 & 一 \\
\hline $\begin{array}{l}\text { Galactic center } \\
\text { (HESS, EHECR) }\end{array}$ & 2.7 & 2.6 & 2.3 & 1.7 & - \\
\hline Galactic plane & 0.21 & 0.2 & 0.17 & 0.12 & - \\
\hline $\begin{array}{l}\text { Galactic } \quad \text { center } \\
\text { (Candia) }\end{array}$ & 650 & 630 & 540 & 410 & - \\
\hline $\begin{array}{l}\text { Galactic latitude } 45^{\circ} \\
\text { (Candia) }\end{array}$ & 170 & 160 & 140 & 100 & - \\
\hline GBBs (De Paolis) & 0.77 & 0.77 & 0.7 & 0.55 & 4.3 \\
\hline GRB941017 & 0.0013 & 0.002 & 0.0034 & 0.005 & - \\
\hline $\begin{array}{l}\text { GRB flux limit } \\
(\mathrm{MPR})\end{array}$ & 900 & 910 & 820 & 650 & 5100 \\
\hline $\begin{array}{l}\text { GRB flux limit } \\
\text { (WB) }\end{array}$ & 0.089 & 0.11 & 0.13 & 0.14 & 0.8 \\
\hline GRB afterglow & 1.5 & 2.5 & 5.6 & 33 & 53 \\
\hline AGN jet & $1.1 \times 10^{-5}$ & $1.7 \times 10^{-5}$ & $3.2 \times 10^{-5}$ & 0.00011 & 0.00025 \\
\hline $3 \mathrm{C} 273(p p)$ & 4.3 & 4.5 & 4.2 & 3.4 & - \\
\hline $3 \mathrm{C} 273(p \gamma)$ & 0.03 & 0.044 & 0.073 & 0.12 & - \\
\hline radio quiet $\mathrm{AGN}$ & 0.29 & 0.34 & 0.39 & 0.38 & - \\
\hline $\begin{array}{l}\text { FR-II radio galaxies } \\
\text { and blazars }\end{array}$ & 62 & 63 & 58 & 48 & 360 \\
\hline $1 \mathrm{ES} 1959+650$ & 1.5 & 1.6 & 1.5 & 1.2 & - \\
\hline $\begin{array}{l}\text { black hole forma- } \\
\text { tion (cluster formed } \\
\text { early) }\end{array}$ & 16 & 16 & 15 & 12 & - \\
\hline $\begin{array}{l}\text { black hole formation } \\
\text { (cluster formed late) }\end{array}$ & 130 & 140 & 130 & 110 & - \\
\hline galaxy clusters & 0.64 & 0.65 & 0.61 & 0.5 & 3.8 \\
\hline upper MPR limit & 410 & 420 & 390 & 320 & 2400 \\
\hline lower MPR limit & 280 & 280 & 260 & 210 & 1600 \\
\hline WB limit & 11 & 11 & 11 & 8.7 & 65 \\
\hline AMANDA limit & 160 & 160 & 150 & 120 & 930 \\
\hline
\end{tabular}

Table B.5: Total tauon event rates $\dot{N}_{\tau}$ at various nadir angles $\theta$ for the astrophysical sources discussed in Chapter 5. A detector energy offset of $100 \mathrm{GeV}$ is assumed. In case of the isotropic source spectra, the integral $\dot{N}_{\tau, 2 \pi}$ of the total event rate over all nadir angles from $0^{\circ}$ to $90^{\circ}$ is given as well. Fluences have been divided by 1 yr. 
164 APPENDIX B. EVENT RATES AND Z FACTOR INACCURACIES

\begin{tabular}{|c|c|c|c|c|c|}
\hline source & $\begin{array}{l}\dot{N}_{\tau}(\theta)(y \\
\theta=0^{\circ}\end{array}$ & $\begin{array}{r}\left.-1\left(\mathrm{sr}^{-1}\right)\right) \\
\theta=30^{\circ}\end{array}$ & $\begin{array}{c}\text { no rock col } \\
\theta=60^{\circ}\end{array}$ & $\begin{array}{l}\text { rection] } \\
\theta=90^{\circ}\end{array}$ & $\dot{N}_{\tau, 2 \pi}\left(\mathrm{yr}^{-1}\right)$ \\
\hline RX J1713.7-3946 & 2 & 2 & 2.2 & 2.3 & - \\
\hline Crab & 0.4 & 0.42 & 0.44 & 0.45 & 一 \\
\hline shocked pulsar wind & 0.27 & 0.32 & 0.38 & 0.43 & - \\
\hline SS433 & 160 & 180 & 200 & 230 & - \\
\hline SGR 1806-20 & 150 & 160 & 180 & 210 & - \\
\hline Cyg OB2 & 3.8 & 3.7 & 3.6 & 3.5 & - \\
\hline $\begin{array}{l}\text { Galactic center } \\
\text { (HESS, EHECR) }\end{array}$ & 1.6 & 1.6 & 1.7 & 1.7 & - \\
\hline Galactic plane & 0.12 & 0.12 & 0.12 & 0.12 & - \\
\hline $\begin{array}{l}\text { Galactic } \quad \text { center } \\
\text { (Candia) }\end{array}$ & 390 & 390 & 400 & 410 & - \\
\hline $\begin{array}{l}\text { Galactic latitude } 45^{\circ} \\
\text { (Candia) }\end{array}$ & 99 & 100 & 100 & 100 & - \\
\hline GBBs (De Paolis) & 0.46 & 0.48 & 0.52 & 0.55 & 3.2 \\
\hline GRB941017 & 0.00079 & 0.0013 & 0.0025 & 0.005 & - \\
\hline $\begin{array}{l}\text { GRB flux limit } \\
\text { (MPR) }\end{array}$ & 530 & 570 & 610 & 650 & 3800 \\
\hline $\begin{array}{lll}\text { GRB } & \text { flux } & \text { limit } \\
\text { (WB) } & & \end{array}$ & 0.053 & 0.069 & 0.098 & 0.14 & 0.62 \\
\hline GRB afterglow & 0.91 & 1.6 & 4.2 & 33 & 46 \\
\hline AGN jet & $6.7 \times 10^{-6}$ & $1.1 \times 10^{-5}$ & $2.4 \times 10^{-5}$ & 0.00011 & 0.00021 \\
\hline $3 \mathrm{C} 273(p p)$ & 2.6 & 2.8 & 3.1 & 3.4 & - \\
\hline $3 \mathrm{C} 273(p \gamma)$ & 0.018 & 0.027 & 0.054 & 0.12 & 一 \\
\hline radio quiet AGN & 0.17 & 0.21 & 0.29 & 0.38 & - \\
\hline $\begin{array}{l}\text { FR-II radio galaxies } \\
\text { and blazars }\end{array}$ & 37 & 39 & 43 & 48 & 270 \\
\hline 1ES $1959+650$ & 0.91 & 0.98 & 1.1 & 1.2 & - \\
\hline $\begin{array}{l}\text { black hole forma- } \\
\text { tion (cluster formed } \\
\text { early) }\end{array}$ & 9.2 & 9.9 & 11 & 12 & - \\
\hline $\begin{array}{l}\text { black hole formation } \\
\text { (cluster formed late) }\end{array}$ & 78 & 85 & 97 & 110 & - \\
\hline galaxy clusters & 0.38 & 0.41 & 0.46 & 0.5 & 2.8 \\
\hline upper MPR limit & 240 & 260 & 290 & 320 & 1800 \\
\hline lower MPR limit & 160 & 180 & 200 & 210 & 1200 \\
\hline WB limit & 6.6 & 7.1 & 7.9 & 8.7 & 49 \\
\hline AMANDA limit & 94 & 100 & 110 & 120 & 700 \\
\hline
\end{tabular}

Table B.6: Total tauon event rates $\dot{N}_{\tau}$ at various nadir angles $\theta$ for the astrophysical sources discussed in Chapter 5, if the rock correction factor (Eq. B.1) is ignored. The vales are normalized, so that they agree with those of Table B.5 at $\theta=90^{\circ}$. A detector energy offset of $100 \mathrm{GeV}$ is assumed. In case of the isotropic source spectra, the integral $\dot{N}_{\tau, 2 \pi}$ of the total event rate over all nadir angles from $0^{\circ}$ to $90^{\circ}$ is given as well. Fluences have been divided by $1 \mathrm{yr}$. 


\begin{tabular}{|c|c|c|c|c|c|}
\hline \multirow[t]{2}{*}{ source } & \multicolumn{4}{|c|}{$\dot{N}_{\tau}(\theta)\left(\mathrm{yr}^{-1}\left(\mathrm{sr}^{-1}\right)\right)$} & \multirow[t]{2}{*}{$\dot{N}_{\tau, 2 \pi}\left(\mathrm{yr}^{-1}\right)$} \\
\hline & $\theta=0^{\circ}$ & $\theta=30^{\circ}$ & $\theta=60^{\circ}$ & $\theta=90^{\circ}$ & \\
\hline RX J1713.7-3946 & 1.2 & 1.3 & 1.2 & 1 & - \\
\hline Crab & 0.35 & 0.36 & 0.33 & 0.26 & - \\
\hline shocked pulsar wind & 0.39 & 0.43 & 0.44 & 0.38 & - \\
\hline $\mathrm{SS} 433$ & 210 & 220 & 220 & 200 & - \\
\hline SGR 1806-20 & 140 & 150 & 150 & 130 & - \\
\hline Cyg OB2 & 0.39 & 0.38 & 0.33 & 0.25 & 一 \\
\hline $\begin{array}{l}\text { Galactic center } \\
\text { (HESS, EHECR) }\end{array}$ & 0.4 & 0.43 & 0.42 & 0.35 & - \\
\hline Galactic plane & 0.0044 & 0.0043 & 0.0038 & 0.0029 & - \\
\hline $\begin{array}{l}\text { Galactic } \quad \text { center } \\
\text { (Candia) }\end{array}$ & 120 & 120 & 110 & 89 & - \\
\hline $\begin{array}{l}\text { Galactic latitude } 45^{\circ} \\
\text { (Candia) }\end{array}$ & 26 & 26 & 23 & 18 & - \\
\hline GBBs (De Paolis) & 0.35 & 0.37 & 0.36 & 0.3 & 2.2 \\
\hline GRB941017 & 0.0013 & 0.002 & 0.0034 & 0.0049 & - \\
\hline $\begin{array}{l}\text { GRB flux limit } \\
(\mathrm{MPR})\end{array}$ & 420 & 450 & 440 & 360 & 2700 \\
\hline $\begin{array}{l}\text { GRB flux limit } \\
\text { (WB) }\end{array}$ & 0.083 & 0.1 & 0.13 & 0.13 & 0.77 \\
\hline GRB afterglow & 1.5 & 2.5 & 5.6 & 33 & 53 \\
\hline AGN jet & $1.1 \times 10^{-5}$ & $1.7 \times 10^{-5}$ & $3.2 \times 10^{-5}$ & 0.0001 & 0.00025 \\
\hline $3 \mathrm{C} 273(p p)$ & 2.5 & 2.8 & 2.8 & 2.3 & - \\
\hline $3 \mathrm{C} 273(p \gamma)$ & 0.029 & 0.043 & 0.072 & 0.12 & - \\
\hline radio quiet $\mathrm{AGN}$ & 0.26 & 0.31 & 0.37 & 0.36 & - \\
\hline $\begin{array}{l}\text { FR-II radio galaxies } \\
\text { and blazars }\end{array}$ & 31 & 33 & 33 & 29 & 210 \\
\hline $1 \mathrm{ES} 1959+650$ & 0.77 & 0.83 & 0.83 & 0.73 & - \\
\hline $\begin{array}{l}\text { black hole forma- } \\
\text { tion (cluster formed } \\
\text { early) }\end{array}$ & 7.8 & 8.4 & 8.4 & 7.4 & - \\
\hline $\begin{array}{l}\text { black hole formation } \\
\text { (cluster formed late) }\end{array}$ & 73 & 80 & 82 & 74 & - \\
\hline galaxy clusters & 0.33 & 0.36 & 0.36 & 0.31 & 2.2 \\
\hline upper MPR limit & 210 & 220 & 220 & 200 & 1400 \\
\hline lower MPR limit & 160 & 170 & 170 & 140 & 1000 \\
\hline WB limit & 5.6 & 6 & 6 & 5.3 & 37 \\
\hline AMANDA limit & 80 & 86 & 86 & 75 & 530 \\
\hline
\end{tabular}

Table B.7: Total tauon event rates $\dot{N}_{\tau}$ at various nadir angles $\theta$ for the astrophysical sources discussed in Chapter 5. A detector energy offset of $1000 \mathrm{GeV}$ is assumed. In case of the isotropic source spectra, the integral $\dot{N}_{\tau, 2 \pi}$ of the total event rate over all nadir angles from $0^{\circ}$ to $90^{\circ}$ is given as well. Fluences have been divided by 1 yr. 
166 APPENDIX B. EVENT RATES AND Z FACTOR INACCURACIES

\begin{tabular}{|c|c|c|c|c|c|}
\hline source & $\begin{array}{c}\dot{\boldsymbol{N}}_{\tau}(\theta)(\mathrm{yl} \\
\theta=0^{\circ}\end{array}$ & $\begin{array}{l}\left.{ }^{-1}\left(\mathrm{sr}^{-1}\right)\right) \\
\theta=30^{\circ}\end{array}$ & $\begin{array}{c}\text { [no rock co } \\
\theta=60^{\circ}\end{array}$ & $\begin{array}{r}\text { rection] } \\
\theta=90^{\circ}\end{array}$ & $\dot{N}_{\tau, 2 \pi}\left(\mathrm{yr}^{-1}\right)$ \\
\hline RX J1713.7-3946 & 0.72 & 0.8 & 0.9 & 1 & - \\
\hline Crab & 0.21 & 0.22 & 0.24 & 0.26 & - \\
\hline shocked pulsar wind & 0.23 & 0.27 & 0.33 & 0.38 & - \\
\hline SS433 & 120 & 140 & 170 & 200 & - \\
\hline SGR 1806-20 & 81 & 93 & 110 & 130 & - \\
\hline Cyg OB2 & 0.23 & 0.24 & 0.25 & 0.25 & - \\
\hline $\begin{array}{l}\text { Galactic center } \\
\text { (HESS, EHECR) }\end{array}$ & 0.24 & 0.27 & 0.31 & 0.35 & - \\
\hline Galactic plane & 0.0026 & 0.0027 & 0.0028 & 0.0029 & - \\
\hline $\begin{array}{l}\text { Galactic } \quad \text { center } \\
\text { (Candia) }\end{array}$ & 72 & 78 & 84 & 89 & - \\
\hline $\begin{array}{l}\text { Galactic latitude } 45^{\circ} \\
\text { (Candia) }\end{array}$ & 15 & 16 & 17 & 18 & - \\
\hline GBBs (De Paolis) & 0.21 & 0.23 & 0.27 & 0.3 & 1.7 \\
\hline GRB941017 & 0.00078 & 0.0012 & 0.0025 & 0.0049 & - \\
\hline $\begin{array}{l}\text { GRB flux limit } \\
\text { (MPR) }\end{array}$ & 250 & 280 & 330 & 360 & 2000 \\
\hline $\begin{array}{l}\text { GRB flux limit } \\
(\mathrm{WB})\end{array}$ & 0.049 & 0.065 & 0.095 & 0.13 & 0.6 \\
\hline GRB afterglow & 0.9 & 1.6 & 4.2 & 33 & 45 \\
\hline AGN jet & $6.5 \times 10^{-6}$ & $10^{-5}$ & $2.4 \times 10^{-5}$ & 0.0001 & 0.00021 \\
\hline $3 \mathrm{C} 273(p p)$ & 1.5 & 1.7 & 2.1 & 2.3 & - \\
\hline $3 \mathrm{C} 273(p \gamma)$ & 0.017 & 0.027 & 0.054 & 0.12 & - \\
\hline radio quiet AGN & 0.15 & 0.2 & 0.27 & 0.36 & - \\
\hline $\begin{array}{l}\text { FR-II radio galaxies } \\
\text { and blazars }\end{array}$ & 18 & 21 & 25 & 29 & 160 \\
\hline 1ES 1959+650 & 0.46 & 0.52 & 0.62 & 0.73 & - \\
\hline $\begin{array}{l}\text { black hole forma- } \\
\text { tion (cluster formed } \\
\text { early) }\end{array}$ & 4.6 & 5.3 & 6.3 & 7.4 & - \\
\hline $\begin{array}{l}\text { black hole formation } \\
\text { (cluster formed late) }\end{array}$ & 44 & 50 & 61 & 74 & - \\
\hline galaxy clusters & 0.2 & 0.22 & 0.27 & 0.31 & 1.7 \\
\hline upper MPR limit & 120 & 140 & 170 & 200 & 1000 \\
\hline lower MPR limit & 95 & 110 & 130 & 140 & 780 \\
\hline WB limit & 3.3 & 3.8 & 4.5 & 5.3 & 28 \\
\hline AMANDA limit & 47 & 54 & 64 & 75 & 400 \\
\hline
\end{tabular}

Table B.8: Total tauon event rates $\dot{N}_{\tau}$ at various nadir angles $\theta$ for the astrophysical sources discussed in Chapter 5, if the rock correction factor (Eq. B.1) is ignored. The vales are normalized, so that they agree with those of Table B.7 at $\theta=90^{\circ}$. A detector energy offset of $1000 \mathrm{GeV}$ is assumed. In case of the isotropic source spectra, the integral $\dot{N}_{\tau, 2 \pi}$ of the total event rate over all nadir angles from $0^{\circ}$ to $90^{\circ}$ is given as well. Fluences have been divided by $1 \mathrm{yr}$. 


\begin{tabular}{|l|lll|}
\hline source & \multicolumn{3}{|c|}{$(\boldsymbol{\Delta} \boldsymbol{Z} / \boldsymbol{Z})_{\max }$} \\
& $\boldsymbol{\nu}_{\boldsymbol{e}}$ & $\boldsymbol{\nu}_{\boldsymbol{\mu}}$ & $\boldsymbol{\nu}_{\boldsymbol{\tau}}$ \\
\hline RX J1713.7-3946 & 0.00084 & 0.0008 & 0.0013 \\
Crab & $6.3 \times 10^{-6}$ & $6.2 \times 10^{-6}$ & $2.2 \times 10^{-5}$ \\
shocked pulsar wind & $10^{-5}$ & $10^{-5}$ & $3.9 \times 10^{-5}$ \\
SS433 & 0.0017 & 0.0017 & 0.0025 \\
SGR 1806-20 & 0.0017 & 0.0017 & 0.0029 \\
Cyg OB2 & $1.7 \times 10^{-6}$ & $2.5 \times 10^{-5}$ & $7.6 \times 10^{-6}$ \\
Galactic center (HESS, EHECR) & $8.5 \times 10^{-7}$ & $8.7 \times 10^{-7}$ & $7.4 \times 10^{-7}$ \\
Galactic plane & $3.7 \times 10^{-5}$ & $3.7 \times 10^{-5}$ & 0.0002 \\
Galactic center (Candia) & $6.9 \times 10^{-5}$ & $6.8 \times 10^{-5}$ & 0.00046 \\
Galactic latitude 45 (Candia) & $7.8 \times 10^{-5}$ & $7.8 \times 10^{-5}$ & 0.0006 \\
GBBs (De Paolis) & $7.7 \times 10^{-5}$ & $7.7 \times 10^{-5}$ & 0.00058 \\
GRB941017 & 0.002 & 0.0019 & 0.008 \\
GRB flux limit (MPR) & 0.00045 & 0.00043 & 0.0026 \\
GRB flux limit (WB) & $7.8 \times 10^{-5}$ & $7.8 \times 10^{-5}$ & 0.0016 \\
GRB afterglow & 0.047 & 0.047 & 0.062 \\
AGN jet & 0.0051 & 0.0051 & 0.014 \\
3C273 (pp) & 0.0017 & 0.0017 & 0.0025 \\
3C273 ( $p \gamma$ ) & 0.0012 & 0.0012 & 0.0043 \\
radio quiet AGN & $9.8 \times 10^{-5}$ & $9.8 \times 10^{-5}$ & 0.0001 \\
FR-II radio galaxies and blazars & 0.00035 & 0.00036 & 0.0048 \\
1ES 1959+650 & 0.0017 & 0.0017 & 0.0025 \\
black hole formation (cluster formed early) & 0.0017 & 0.0017 & 0.0025 \\
black hole formation (cluster formed late) & 0.0017 & 0.0017 & 0.0025 \\
galaxy clusters & 0.001 & 0.00097 & 0.0017 \\
upper MPR limit & 0.0017 & 0.0017 & 0.0025 \\
lower MPR limit & 0.0044 & 0.0043 & 0.029 \\
WB limit & 0.0017 & 0.0017 & 0.0025 \\
AMANDA limit & 0.0017 & 0.0017 & 0.0025 \\
Gen(0.5,1.0) & 0.00016 & 0.00015 & 0.0012 \\
Gen(0.5,1.5) & $8.1 \times 10^{-5}$ & $8.1 \times 10^{-5}$ & 0.00062 \\
Gen(1.0,0.5) & 0.00016 & 0.00015 & 0.0012 \\
Gen(1.0,2.0) & $3.6 \times 10^{-5}$ & $3.5 \times 10^{-5}$ & 0.00019 \\
\hline
\end{tabular}

Table B.9: Maximum relative inaccuracy of the $Z$ factor for energies between $10^{4}$ and $10^{8} \mathrm{GeV}$ and nadir angles between $0^{\circ}$ and $90^{\circ}$. The given values are averages of the respective inaccuracies for neutrinos and antineutrinos. 
168 APPENDIX B. EVENT RATES AND Z FACTOR INACCURACIES 


\section{Bibliography}

[1] J. N. Abdurashitov et al. Physics Letters B, 328:234-248, 1994.

[2] M. Ackermann et al. Astroparticle Physics, 22:339-353, 2005.

[3] F. Aharonian et al. Astronomy and Astrophysics, 393:L37-L40, 2002.

[4] F. Aharonian et al. Astronomy and Astrophysics, 425:L13-L17, 2004.

[5] Q. R. Ahmad et al. Physical Review Letters, 89:011301, 2002.

[6] J. Ahrens et al. Physical Review D, 67:012003, 2003.

[7] J. Ahrens et al. New Astronomy Reviews, 48:519-525, 2004.

[8] E. K. Akhmedov, M. A. Tórtola, and J. W. F. Valle. Journal of High Energy Physics, 6/2005:53, 2005.

[9] M. Z. Akrawy et al. Physics Letters B, 240:497-512, 1990.

[10] V. Albul et al. Instruments and Experimental Techniques, 44:327-334, 2001.

[11] E. N. Alexeyev et al. Physics Letters B, 205:209-214, 1988.

[12] C. J. Allègre et al. Earth and Planetary Science Letters, 134:515-526, 1995.

[13] G. Altarelli and G. Parisi. Nuclear Physics B, 126:298-318, 1977.

[14] J. Alvarez-Muñiz and F. Halzen. The Astrophysical Journal, 576:L33L36, 2002. 
[15] J. Alvarez-Muñiz, F. Halzen, and D. Hooper. The Astrophysical Journal, 604:L85-L88, 2004.

[16] J. Alvarez-Muñiz and P. Mészáros. Physical Review D, 70:123001, 2004.

[17] J. Alvarez-Muñiz and E. Zas. Prospects for radio detection of extremely high energy cosmic rays and neutrinos in the Moon. In D. Saltzberg and P. Gorham, editors, Radio Detection of High Energy Particles: First International Workshop; RADHEP 2000, number 579 in AIP Conference Proceedings, pages 128-138. American Institute of Physics, 2001.

[18] L. A. Anchordoqui et al. Physics Letters B, 593:42-47, 2004.

[19] E. Andrés et al. Astroparticle Physics, 13:1-20, 2000.

[20] E. Andrés et al. Nature, 410:441-443, 2001.

[21] P. Anselmann et al. Physics Letters B, 285:376-389, 1992.

[22] P. L. Anthony et al. Physical Review Letters, 75:1949-1952, 1995.

[23] C. Aramo et al. Astroparticle Physics, 23:65-77, 2005.

[24] G. A. Askar'yan. Soviet Physics JETP, 14:441-443, 1962.

[25] G. A. Askar'yan. Soviet Physics JETP, 21:658-659, 1965.

[26] H. Athar et al. European Physical Journal C, 33:S959-S961, 2004.

[27] H. Athar, M. Jeżabek, and O. Yasuda. Physical Review D, 62:103007, 2000 .

[28] M. Ave et al. Astroparticle Physics, 23:19-29, 2005.

[29] J. N. Bahcall and P. Mészáros. Physical Review Letters, 85:1362-1365, 2000 .

[30] H. Bahlburg and C. Breitkreuz. Grundlagen der Geologie. Enke, 1998.

[31] R. Bailey et al. The CERN neutrino beam to Gran Sasso (NGS). Technical Report CERN-SL/99-034(DI), CERN, 1999. 
[32] Y. Y. Balitskiŭ and L. N. Lipatov. Soviet Journal of Nuclear Physics, 28:822-829, 1978.

[33] R. M. Baltrusaitis et al. Physical Review D, 31:2192-2198, 1985.

[34] J. F. Beacom, P. Crotty, and E. W. Kolb. Physical Review D, 66:021302, 2002.

[35] J. K. Becker, P. L. Biermann, and W. Rhode. Astroparticle Physics, 23:355-368, 2005.

[36] R. Becker-Szendy. Nuclear Instruments and Methods in Physics Research Section A, 324:363-382, 1993.

[37] W. Bednarek and R. J. Protheroe. Physical Review Letters, 79:26162619, 1997.

[38] V. S. Berezinsky and V. I. Dokuchaev. Astroparticle Physics, 15:87-96, 2001.

[39] V. S. Berezinsky and V. I. Dokuchaev. The Astrophysical Journal (submitted), 2004.

[40] L. Bergström, J. Edsjö, and P. Gondolo. Physical Review D, 58:103519, 1998.

[41] S. M. Bilenky. Basics of Introduction to Feynman Diagrams and Electroweak Interactions Physics. Editions Frontières, 1994.

[42] S. M. Bilenky, C. Giunti, and W. Grimus. Progress in Particle and Nuclear Physics, 43:1-86, 1999.

[43] M. Birkel and S. Sarkar. Astroparticle Physics, 9:297-309, 1998.

[44] R. J. Blin-Stoyle. Nuclear and Particle Physics, volume 4 of Physics and its Applications. Chapman \& Hall, London, 1991.

[45] F. Boehm and P. Vogel. Physics of Massive Neutrinos. Cambridge University Press, Cambridge, second edition, 1992.

[46] J. Boger et al. Nuclear Instruments and Methods in Physics Research Section A, 449:172-207, 2000. 
[47] C. B. Bratton et al. Physical Review D, 37:3361-3363, 1988.

[48] L. M. Brown. Physics Today, 9/1978:23-28, 1978.

[49] E. Bugaev et al. Astroparticle Physics, 21:491-509, 2004.

[50] J. Candia. astro-ph/0505346, 2005.

[51] K. S. Capelle et al. Astroparticle Physics, 8:321-328, 1998.

[52] G. Cavallo and M. J. Rees. Monthly Notices of the Royal Astronomical Society, 183:359-365, 1978.

[53] http://www.cgicc.org.

[54] S. Colafrancesco and P. Blasi. Astroparticle Physics, 9:227-246, 1998.

[55] E. Costa et al. Nature, 387:783-785, 1997.

[56] M. L. Costantini and F. Vissani. Astroparticle Physics, 23:477-485, 2005 .

[57] R. M. Crocker, F. Melia, and R. R. Volkas. The Astrophysical Journal Letters, 622:37-40, 2005.

[58] http://www.phys.psu.edu/ cteq/.

[59] http://user.pa.msu.edu/wkt/cteq/cteq6/cteq6pdf.html.

[60] G. Danby et al. Physical Review Letters, 9:36-44, 1962.

[61] R. Davis, D. S. Harmer, and K. C. Hoffman. Physical Review Letters, 20:1205-1209, 1968.

[62] S. R. Deans. The Radon transform and some of its applications. Krieger Pub. Co, 1993.

[63] C. Distefano et al. The Astrophysical Journal, 575:378-383, 2002.

[64] Z. Djilkibaev. Light propagation in water and ice. In C. Spiering, editor, Proceedings of the Workshop on Simulation and Analysis Methods for Large Neutrino Telescopes, pages 132-144. DESY Zeuthen, 1998.

[65] S. G. Djorgovski et al. Nature, 387:876-878, 1997. 
[66] S. I. Dutta et al. Physical Review D, 63:094020, 2001.

[67] S. I. Dutta, Y. Huang, and M. H. Reno. Physical Review D, 72:013005, 2005 .

[68] S. I. Dutta, M. H. Reno, and I. Sarcevic. Physical Review D, 62:123001, 2000 .

[69] A. M. Dziewonski and D. L. Anderson. Physics of the Earth and Planetary Interiors, 25:297-356, 1981.

[70] S. Eidelman et al. Physics Letters B, 592:1, 2004.

[71] J. Ellis. Nuclear Physics A, 663:231-248, 2000.

[72] R. K. Ellis, W. J. Stirling, and B. R. Webber. QCD and collider physics. Cambridge University Press, 1996.

[73] R. Enomoto et al. Nature, 416:823-826, 2002.

[74] http://www.netlib.org/f2c/.

[75] V. S. Fadin, E. A. Kuraev, and L. N. Lipatov. Physics Letters B, 60:50-52, 1975.

[76] D. Fargion. The Astrophysical Journal, 570:909-925, 2002.

[77] D. Fargion et al. astro-ph/0501033, 2005.

[78] B. D. Fields and K. A. Hochmuth. hep-ph/0406001, 2004.

[79] G. Fishman and C. A. Meegan. Annual Review of Astronomy and Astrophysics, 33:415-458, 1995.

[80] Y. Fukuda et al. Physical Review Letters, 81:4279, 1998.

[81] Y. Fukuda et al. Physical Review Letters, 81:1562-1567, 1998.

[82] Y. Fukuda et al. Physical Review Letters, 81:1158-1162, 1998.

[83] T. K. Gaisser. Cosmic Rays and Particle Physics. Cambridge University Press, Cambridge, 1990. 
[84] C. C. Gaither and A. E. Cavazos-Gaither. Physically speaking: A Dictionary of Quotations on Physics and Astronomy. Institute of Physics Publishing, Bristol and Philadelphia, 1997.

[85] G. Gamow. Physics Today, 07/1948:4, 1948.

[86] R. Gandhi et al. Astroparticle Physics, 5:81-110, 1996.

[87] R. Gandhi et al. Physical Review D, 58:093009, 1998.

[88] R. Genzel et al. Nature, 425:934-937, 2003.

[89] http://www.gnuplot.info/.

[90] H. Goenner. Einführung in die Kosmologie. Spektrum Akademischer Verlag, Heidelberg, 1994.

[91] M. C. Gonzalez-Garcia, F. Halzen, and M. Maltoni. Physical Review D, 71:093010, 2005.

[92] P. W. Gorham. Astroparticle Physics, 15:177-202, 2001.

[93] D. E. Groom et al. European Physical Journal C, 15:1, 2000.

[94] C. Grupen. Astroteilchenphysik. Friedrich Vieweg \& Sohn Verlagsgesellschaft mbH, Braunschweig/Wiesbaden, 2000.

[95] http://www.gnu.org/software/gsl/.

[96] F. Halzen and D. W. Hooper. Astroparticle Physics, 23:537-542, 2005.

[97] J. Hauptman. On the detection of UHE cascade showers with DUMAND II. In D. A. Leahy, editor, Proceedings of the 23rd International Cosmic Ray Conference, 1993.

[98] C. Hettlage and K. Mannheim. The sun as a high-energy neutrino source. In Trân Thanh Vân, editor, Proceedings of the XIth Rencontres de Blois, pages 515-517. The Gioi Publishers, 2001.

[99] C. Hettlage and K. Mannheim. Nuclear Physics B Proceedings Supplements, 95:165-168, 2001. 
[100] C. Hettlage and K. Mannheim. Neutrino flux bounds and prospects for high energy and ultrahigh energy neutrino source detection. In R. Wischnewski, editor, 2nd Workshop on Methodical Aspects of Underwater/Ice Neutrino Telescopes, pages 47-51, 2002.

[101] C. Hettlage and K. Mannheim. High energy neutrino fluxes from cosmic accelerators. In M. Boër and J. Trân Thanh Vân, editors, Proceedings of the XXXVIth Rencontres de Moriond, pages 311-314. The Gioi Publishers, 2003.

[102] C. Hettlage and K. Mannheim. Nuclear Physics B Proceedings Supplements, 118:517, 2003.

[103] C. Hettlage, K. Mannheim, and J. G. Learned. Astroparticle Physics, 13:45-50, 2000.

[104] K. S. Hirata et al. Physical Review D, 38:448-458, 1988.

[105] D. Hooper, A. Taylor, and S. Sarkar. Astroparticle Physics, 23:11-17, 2005.

[106] K. Hurley et al. Nature, 434:1098-1103, 2005.

[107] http://vassilo.home.cern.ch/vassilo/NGS/ngs99ccxs.html.

[108] G. Ingelman and M. Thunman. Physical Review D, 54:4385-4392, 1996.

[109] K. Ioka. Monthly Notices of the Royal Astronomical Society, 327:639$662,2001$.

[110] K. Ioka et al. The Astrophysical Journal (accepted), 2005.

[111] J. D. Jackson. Classical Electrodynamics. Wiley, New York, 1999.

[112] P. Jain, J. P. Ralston, and G. M. Frichter. Astroparticle Physics, 12:193-198, 1999.

[113] T. Kashti and E. Waxman. astro-ph/0507599, 2005.

[114] A. K. Kembhavi and J. V. Narlika. Quasars and active galactic nuclei: an introduction. Cambridge University Press, Cambridge, 1999. 
[115] B. L. N. Kennett. Geophysical Journal International, 132:374-382, 1998.

[116] C. R. Kitchin. Astrophysical Techniques. Institute of Physics Publishing, Bristol, 1998.

[117] R. W. Klebesadel, I. B. Strong, and R. A. Olson. The Astrophysical Journal, 182:L85-L88, 1973.

[118] S. I. Klimushin, E. V. Bugaev, and I. A. Sokalski. Precise parametrizations of muon energy losses in water. In Proceedings of the 27th International Cosmic Ray Conference. 07-15 August, 2001. Hamburg, Germany, page 1009, 2001.

[119] J. Knödlseder. Astronomy and Astrophysics, 360:539-548, 2000.

[120] K. Kodama et al. Physics Letters B, 504:218-224, 2001.

[121] I. Kravchenko et al. Astroparticle Physics, 20:195-213, 2003.

[122] I. Kravchenko et al. Astroparticle Physics, 19:15-36, 2003.

[123] E. A. Kuraev, L. N. Lipatov, and V. S. Fadin. Soviet Physics JETP, 44:443-451, 1976.

[124] E. A. Kuraev, L. N. Lipatov, and V. S. Fadin. Soviet Physics JETP, 45:199-204, 1977.

[125] H. L. Lai et al. Physical Review D, 55:1280-1296, 1997.

[126] J. G. Learned. Physical Review D, 19:3293-3307, 1979.

[127] J. G. Learned and K. Mannheim. Annual Reviews of Nuclear and Particle Science, 50:679-749, 2000.

[128] J. G. Learned and S. Pakvasa. Astroparticle Physics, 3:267-274, 1995.

[129] N. Lehtinen et al. Astroparticle Physics, 17:279-292, 2002.

[130] W. R. LePage. Complex variables and the Laplace transform for engineers. Dover Publ., New York, 1980.

[131] A. Levinson and E. Waxman. Physical Review Letters, 87:171101, 2001. 
[132] J. Linder. hep-ph/0505079, 2005.

[133] L. N. Lipatov. Soviet Physics JETP, 63:904-912, 1986.

[134] M. S. Longair. High Energy Astrophysics, volume 1. Cambridge University Press, Cambridge, second edition, 1992.

[135] M. Maltoni et al. New Journal of Physics, 6:122, 2004.

[136] K. Mannheim. Astronomy and Astrophysics, 269:67-76, 1993.

[137] K. Mannheim. Astroparticle Physics, 3:295-302, 1995.

[138] K. Mannheim. Neutrinos from gamma-ray bursts. In F. A. Aharonian and H. J. Völk, editors, High Energy Gamma-Ray Astronomy, volume 558 of AIP Conference Proceedings, pages 417-424. American Institute of Physics, 2001.

[139] K. Mannheim, R. J. Protheroe, and J. P. Rachen. Physical Review D, 63:023003, 2001.

[140] B. R. Martin and G. Shaw. Particle Physics. The Manchester Physics Series. John Wiley \& Sons, Chichester, second edition, 1997.

[141] H. A. Mayer-Hasselwander et al. Astronomy and Astrophysics, 335:161$172,1998$.

[142] C. A. Meegan et al. Nature, 355:143-145, 1992.

[143] P. Mészáros. Annual Review of Astronomy and Astrophysics, 40:137169, 2002.

[144] P. Mészáros and E. Waxman. Physical Review Letters, 87:171102, 2001.

[145] M. R. Metzger et al. Nature, 387:878-880, 1997.

[146] A. B. Migdal. Physical Review, 103:1811-1820, 1956.

[147] S. P. Mikheev and A. Y. Smirnov. Soviet Physics Uspekhi, 30:759-790, 1987.

[148] P. Miočinović et al. astro-ph/0503304, 2005. 
[149] I. F. Mirabel and L. F. Rodríguez. Annual Review of Astronomy and Astrophysics, 37:409-443, 1999.

[150] P. M. Morse and H. Feshbach. Methods of Theoretical Physics. McGraw-Hill Book Company, New York, 1953.

[151] A. E. Mussett and M. Aftab Khan. Looking into the Earth: an introduction to geological geophysics. Cambridge University Press, Cambridge, 2000 .

[152] S. Nagataki. The Astrophysical Journal, 600:883-904, 2004.

[153] V. A. Naumov and L. Perrone. Astroparticle Physics, 10:239-252, 1999.

[154] C. E. Navia et al. Physical Review D, 67:103008, 2003.

[155] L. Nellen, K. Mannheim, and P. L. Biermann. Physical Review D, 47:5270-5274, 1993.

[156] A. Nicolaidis and A. Taramopoulos. Physics Letters B, 386:211-216, 1996.

[157] T. Ohlsson and W. Winter. Physics Letters B, 512:357-364, 2001.

[158] L. B. Okun. Leptons and Quarks. North-Holland Publishing Company, 1982.

[159] D. M. Palmer et al. Nature, 434:1107-1109, 2005.

[160] F. De Paolis et al. Astroparticle Physics, 18:249-260, 2003.

[161] L. Pasquali and M. H. Reno. Physical Review D, 59:093003, 1999.

[162] M. L. Perl et al. Physical Review Letters, 35:1489-1492, 1975.

[163] S. Polityko et al. Nuclear Instruments and Methods in Physics Research Section B, 173:30-36, 2001.

[164] T. Pradier. Frascati Physics Series, 555:1-7, 2004.

[165] W. H. Press et al. Numerical recipes in C: the art of scientific computing. Cambridge University Press, Cambridge, second edition, 1992. 
[166] R. J. Protheroe. High energy neutrinos from blazars. In D. T. Wickramasinghe, G. V. Bicknell, and L. Ferrario, editors, Accretion Phenomena and Related Outflows, volume 121 of ASP Conference Series, pages 585-588. IAU, 1997.

[167] http://www.netlib.org/quadpack/.

[168] G. G. Raffelt. Stars as laboratories for fundamental physics: the astrophysics of neutrinos, axions, and other weakly interacting particles. University of Chicago Press, 1996.

[169] F. Reines and C. L. Cowan. Physical Review, 92:30-31, 1953.

[170] E. Resconi. High energy neutrinos as cosmic messengers: AMANDA and IceCube. http://conferences.fnal.gov/aspen05/, 2005. Talk given at the Aspen Winter Conference, 13-19 February 2005: The Highest Energy Physics.

[171] M. M. Reynoso and O. A. Sampayo. Astroparticle Physics, 21:315-324, 2004.

[172] A. Roberts. Reviews of Modern Physics, 64:259-312, 1992.

[173] B. Rossi and K. Greisen. Reviews of Modern Physics, 13:240-309, 1941.

[174] G. B. Rybicki and A. P. Lightman. Radiative Processes in Astrophysics. John Wiley \& Sons, New York, 1979.

[175] D. Saltzberg. astro-ph/0501364, 2005.

[176] M. Sasaki, Y. Asaoka, and M. Jobashi. Astroparticle Physics, 19:37-46, 2003.

[177] N. Schmitz. Neutrinophysik. Teubner, Stuttgart, 1997.

[178] R. Schödel et al. Nature, 419:694-696, 2002.

[179] D. W. Sciama. Modern Cosmology and the Dark Matter Problem, volume 3 of Cambridge Lecture Notes in Physics. Cambridge University Press, Cambridge, 1993.

[180] D. Seckel, T. Stanev, and T. K. Gaisser. The Astrophysical Journal, 382:652-666, 1991. 
[181] T. Sjöstrand. Computer Physics Communications, 82:74-89, 1994.

[182] F. W. Stecker and M. H. Salamon. Space Science Reviews, 75:341-355, 1996.

[183] L. Sulak et al. Nuclear Instruments and Methods in Physics Research, 161:203-217, 1979.

[184] M. Tegmark. hep-ph/0503257, 2005.

[185] C. Thompson and R. C. Duncan. Monthly Notices of the Royal Astronomical Society, 275:255-300, 1995.

[186] D. J. Thompson et al. Journal of Geophysical Research, 102:1473514740, 1997.

[187] M. Thunman, G. Ingelman, and P. Gondolo. Astroparticle Physics, 5:309-332, 1996.

[188] L. V. Volkova. Soviet Journal of Nuclear Physics, 31:784-790, 1980.

[189] E. Waxman. New Journal of Physics, 6:140, 2004.

[190] E. Waxman and J. Bahcall. Physical Review D, 59:023002, 1999.

[191] E. Waxman and J. Bahcall. The Astrophysical Journal, 541:707-711, 2000 .

[192] A. Weigert and H. J. Wendker. Astronomie und Astrophysik: ein Grundkurs. VCH, Weinheim, 1996.

[193] E. W. Weisstein. Entry concerning Wynn's Epsilon Method at http://mathworld.wolfram.com/WynnsEpsilonMethod.html.

[194] T. L. Wilson. Nature, 309:38-42, 1984.

[195] W. Winter. hep-ph/0502097, 2005.

[196] L. Wolfenstein. Physical Review D, 17:2369-2374, 1978.

[197] S. Yoshida. Upper limit of ultra-high energy cosmic neutrino fluxes obtained by Akeno Giant Air Shower Array. In Michel Boër and Jean Trân Thanh Vân, editors, Proceedings of the XXXVIth Rencontres de Moriond, pages 303-309. The Gioi Publishers, 2003. 
[198] E. Zas, F. Halzen, and T. Stanev. Physical Review D, 45:362-376, 1992. 


\section{Acknowledgements}

First of all I would like thank Prof. Dr. Karl Mannheim and Prof. Dr. Klaus Beuermann for all the support and advice they have provided throughout the course of this work. The Studienstiftung des deutschen Volkes, the former Graduiertenkolleg "Strömungsinstabilitäten und Turbulenz", the Göttingen Graduate School of Physics and the Alfried Krupp von Bohlen und HalbachStiftung have provided financial support in form of grants and wages, and I am very grateful for that. My thanks are in addition due to Prof. Dr. Stefan Dreizler for his support.

Looking back, it is a pleasure to see that there are so many people and institutions I have to thank. Let me start with all the colleagues. I am indebted to everybody, but I'd like to mention some in particular: Klaus Reinsch was responsible for the fact that any computer problems I encountered usually didn't last for long. Rick Hessman always showed great enthusiasm when I took some of his valuable time by showing him some new feature of TWiki.XML, and so paying him a visit always proved very motivating. And of course I shouldn't fail to mention all those with whom I had the pleasure to share an office. Thank you for the splendid time together!

Outside the walls of the Institute of Astrophysics, I would like to express my deep gratitude towards all my friends, David Starr, the Katholische Hochschulgemeinde, the Theater im OP, the Theatergruppe der Refo, Anna Radwanska-Nowak and all of the Volkshochschulgruppe for Polish, and many more. My special thanks is also due to all the former and present members of the Fachschaftsrat Physik. Working together has always been fun!

Finally, this work would have been impossible in many ways without the constant support of my parents and the rest of my family. I am very grateful to all of you! 


\section{Curriculum Vitae}

I, Christian Hettlage, was born in Münster/Westfalen on 18 January 1974 as the second child of my parents Maria-Theresia and Heinrich Hettlage.

In 1980 I became pupil of the primary school in Nadorst (Oldenburg). Because of a move, I switched to the primary school in Rhüden am Harz one year later.

From 1984 to 1986 I attended the Orientierungsstufe Seesen. Between 1986 and 1993 I was pupil of the Jacobson-Gymnasium Seesen, where I received my Abitur in May 1993.

In October 1993 I took up my diploma studies in physics at the GeorgAugust-Universität Göttingen, where I passed the Diplomvorprüfung and the Diplomprüfung in June 1995 and May 1998, respectively. My diploma thesis dealt with the investigation of synchrotron self absorption by means of coupled kinetic equations. 